\title{
Discrete And Profinite Groups Acting on Regular Rooted Trees
}

\author{
Dissertation \\ zur Erlangung des mathematisch-naturwissenschaftlichen \\ Doktorgrades \\ "Doctor rerum naturalium" \\ der Georg-August-Universität Göttingen
}

vorgelegt von

Olivier Siegenthaler

aus Lausanne

Göttingen, den 31. August 2009 

Referent: Prof. Dr. Laurent Bartholdi Korreferent: Prof. Dr. Thomas Schick

Tag der mündlichen Prüfung: den 28. September 2009 



\section{Contents}

\begin{tabular}{lr}
\hline Introduction & 1
\end{tabular}

1 Foundations $\quad 5$

1.1 Definition of Aut $X^{*}$ and $\mathcal{A}_{*} \ldots \ldots \ldots \ldots$

1.2 Zariski Topology . . . . . . . . . . . . . . . 7

1.3 Actions of $X^{*} \ldots \ldots \ldots \ldots \ldots \ldots$

1.4 Self-Similarity and Branching . . . . . . . . . . . . . . 9

1.5 Decompositions and Generators of Aut $X^{*}$ and $\mathcal{A}_{*} \ldots \ldots . . .11$

1.6 The Permutation Modules $k\{X\}$ and $k\{\{X\}\} \ldots \ldots . \ldots 13$

1.7 Subgroups of Aut $X^{*} \ldots \ldots \ldots \ldots$. . . . . . . . . . 14

1.8 Regular Branch Groups . . . . . . . . . . . . . . . . . . . . 16

1.9 Self-Similarity and Branching Simultaneously . . . . . . . . . 18

1.10 Questions .......................... 19

2 The Special Case $\operatorname{Aut}_{p} X^{*} \quad 23$

2.1 Definition . . . . . . . . . . . . . . . 23

2.2 Subgroups of Aut $X^{*} \ldots \ldots \ldots \ldots \ldots \ldots$

2.3 Nice Generating Sets . . . . . . . . . . . . . . . . 26

2.4 Uniseriality . . . . . . . . . . . . . . . . . . . . . . 28

2.5 Signature and Maximal Subgroups . . . . . . . . . . . . . . 30

2.6 Torsion-Free Groups . . . . . . . . . . . . . . . . . . . 31

2.7 Some Specific Classes of Automorphisms . . . . . . . . . . . . . . 32

2.8 Torsion Groups . . . . . . . . . . . . . . . . . . . 34

3 Wreath Product of Affine Group Schemes $\quad 37$

3.1 Affine Schemes . . . . . . . . . . . . . . . . . 38

3.2 Exponential Objects . . . . . . . . . . . . . . . . . 40

3.3 Some Hopf Algebra Constructions . . . . . . . . . . . . . . 43

3.4 Group Schemes Corresponding to Aut $X^{*} \ldots$. . . . . . . . . 45

3.5 Iterated Wreath Product of the Frobenius Kernel . . . . . . . . . . 46 
Contents

4 Central Series and Automorphism Towers 49

4.1 Notation . . . . . . . . . . . . . . . . . . . . . . . . 49

4.2 Central Series . . . . . . . . . . . . . . . . . . . . . . . 51

4.3 Automorphism and Normalizer Towers . . . . . . . . . . . . . . 54

5 Hausdorff Dimension $\quad 57$

5.1 Definition . . . . . . . . . . . . . . . . 57

5.2 Layers . . . . . . . . . . . . . . . . . . . . . . 5 58

5.3 Computing Dimensions $\ldots \ldots \ldots$. . . . . . . . . . . 60

5.4 Dimension $1 \ldots \ldots \ldots \ldots \ldots$. . . . . . . . . . . . . . . . . . .

6 Congruence Problems $\quad 65$

6.1 Structure of the Rigid Kernel . . . . . . . . . . . . . . . . 65

6.2 An Example . . . . . . . . . . . . . . . . . . . 67

7 Twisted Twins $\quad 69$

7.1 The Closure of the Grigorchuk Group . . . . . . . . . . . . . . . 69

7.2 Generators and Branchness of $\mathrm{G}_{2} \ldots \ldots \ldots \ldots 71$

7.3 A Presentation of $\mathrm{G}_{2} \ldots \ldots \ldots \ldots 74$

\begin{tabular}{ll}
\hline Appendices & 77
\end{tabular}

$\begin{array}{ll}\text { A Examples } & \mathbf{7 7}\end{array}$

A.1 The Grigorchuk Group . . . . . . . . . . . . . . . . . . . . . . . 77

A.2 The Twisted Twin of the Grigorchuk Group . . . . . . . . . . . . 78

A.3 The Gupta-Sidki Group ．.. . . . . . . . . . . . . . . . . . . . . 79

A.4 The Fabrykowski-Gupta Group . . . . . . . . . . . . . . . . 80

A.5 Another Burnside Group . . . . . . . . . . . . . . . . . . . . 81

A.6 The Binary Adding Machine . . . . . . . . . . . . . 83

A.7 The Brunner-Sidki-Vieira Group . . . . . . . . . . . . . . . . . 84

A.8 The Hanoi Tower Group . . . . . . . . . . . . . . . . . . . 85

B Hausdorff Dimension of Some Groups Acting on the Binary $\begin{array}{ll}\text { Tree } & 87\end{array}$

B.1 Introduction . . . . . . . . . . . . . . . . . . 87

B.2 Hausdorff Dimension . . . . . . . . . . . . . . . . . . . 88

B.3 Spinal Groups . . . . . . . . . . . . . . . . . . . . . . . . 88

B.4 Main Theorem . . . . . . . . . . . . . . . . . . . . . 89

B.5 Finitely Generated Groups of Irrational Hausdorff Dimension . . . 90

B.6 Full-Dimensional Finitely Generated Groups . . . . . . . . . . . . . 91

B.7 Proof of the Main Theorem . . . . . . . . . . . . . . . 93 
C The Congruence Subgroup Problem for Branch Groups $\quad 99$

C.1 Introduction . . . . . . . . . . . . . . . . . . . . . 99

C.2 The Congruence Subgroup Problem . . . . . . . . . . . . . 106

C.3 Examples . . . . . . . . . . . . . . . . . . . . 112

D The Twisted Twin of the Grigorchuk Group 123

D.1 Introduction . . . . . . . . . . . . . . . . . . . 123

D.2 Basic Definitions and Notation . . . . . . . . . . . . . . 124

D.3 Definition of the Group . . . . . . . . . . . . . . . . 125

D.4 A Presentation . . . . . . . . . . . . . . . . . 127

D.5 The Congruence Kernel . . . . . . . . . . . . . . . . . 130

D.6 Germs . . . . . . . . . . . . . . . . . . . . . . 132

D.7 The Lower Central Series . . . . . . . . . . . . . . 136

D.8 The Schur Multiplier . . . . . . . . . . . . . . . . . . . . 138

D.9 GAP Computations . . . . . . . . . . . . . . . . . . 140

$\begin{array}{lr}\text { Bibliography } & 145\end{array}$

$\begin{array}{lr}\text { Curriculum Vitæ } & 149\end{array}$ 



\section{Introduction}

About thirty years ago, people's attention has been attracted to groups acting on rooted trees. For, some particularly simple and elegant constructions of Burnside groups showed up (due to Aleshin [Ale72], Sushchansky [Suš79], and then to Grigorchuk [Gri80] and Gupta and Sidki [GS83a]), as well as the first example of a group of intermediate growth. The "first" Grigorchuk group [Gri83] was the first example, and so far, groups acting on rooted trees remain the only source for such groups.

There has been a number of articles since then, with new examples, generalizations, and so on. Nowadays, it is hard to embrace globally all groups acting on rooted trees, because of their diversity. Apart from the groups mentioned above, there are also many free groups [Bha95, AV05], groups with non-uniformly exponential growth [Wil04], groups which are amenable yet not subexponentially amenable [BV05], ...

Based on the observation that a regular rooted tree contains copies of itself, people considered groups containing (up to finite index) copies of themselves, or groups which almost embed in their own direct powers. From this emerged the definitions of self-similar groups, and of regular branch groups. Both notions express the fact that the group almost replicates itself at each vertex of the tree. Many of the main examples, including Grigorchuk's and Gupta and Sidki's, are both self-similar and regular branch.

For now, the monograph of Nekrashevych [Nek05] is the only one on the topic, and it is oriented towards the correspondence between contracting groups and dynamical systems. We still lack a good reference including the profinite aspects of groups acting on rooted trees, probably because less is known about it. This work is a step in this direction.

\section{Overview}

The first two chapters form the core of this thesis, where we explain in detail our approach to the topic. The following five chapters are mostly independent from one another, and treat several aspects of groups acting on rooted trees, under the light of the core chapters. The first appendix contains a summary of examples treated throughout. It can be used as a reference, and contains a quick introduction to each group. The three publications to which I contributed, and which complement this work, are reprinted as appendices. 


\section{The Core}

We start with the automorphism group Aut $X^{*}$ of a regular $|X|$-ary rooted tree. This is a profinite group when the set $X$ is finite, and we concentrate on that case. We then fix a finite field $k$ and consider the set $\mathcal{A}_{*}$ of all continuous functions Aut $X^{*} \rightarrow k$. This is naturally an infinite dimensional $k$-algebra.

This work is based on a description of closed subgroups $G$ of Aut $X^{*}$ by means of the ideal $\mathcal{I}(G)$ of functions in $\mathcal{A}_{*}$ vanishing on $G$. This reminds of algebraic geometry, and we explain the analogy in detail. Also, we consider only continuous functions Aut $X^{*} \rightarrow k$, that is, functions which factor through a finite quotient of Aut $X^{*}$. Therefore, the ideal $\mathcal{I}(G)$ can be seen as the set of "forbidden patterns" corresponding to $G$, as was introduced in [Gri05] (see also [AHKŠ07, Appendix]).

Identifying the vertices of the tree with the free monoid on $X^{*}$ yields an operation on vertices, corresponding to concatenation of words. In turn, this gives operations on the group Aut $X^{*}$ and dually on $\mathcal{A}_{*}$. From this, we define the crucial notions of branching and self-similarity of groups and of algebras. The group Aut $X^{*}$ and the algebra $\mathcal{A}_{*}$ are not finitely generated, but only finitely many generators are needed when we consider Aut $X^{*}$ and $\mathcal{A}_{*}$ as a branching objects.

As a main object of study, we consider the correspondence between closed subsets of Aut $X^{*}$ and ideals in $\mathcal{A}_{*}$. In particular, closed subgroups correspond to Hopf ideals, and any knowledge about the ideal $\mathcal{I}(G)$ (the annihilator) associated to a closed group $G \leq \operatorname{Aut} X^{*}$ might yields information about $G$. A situation which is particularly well suited to this approach arises when $G$ is regular branch. We shall see that in this case (and only in this case), the ideal $\mathcal{I}(G)$ is finitely generated as a branching ideal. Therefore $G$ is characterized by a finite number of equations or "patterns" [Gri05]. This agrees with a result of Sunic [Šun07].

In Chapter 2 we consider the following special case. Let $X=k=\mathbb{F}_{p}$ be the field with $p$ elements, with $p$ prime. We consider the group $\operatorname{Aut}_{p} X^{*}$ of automorphisms whose activity at each vertex is in the additive group of $\mathbb{F}_{p}$. To a closed subgroup $G$ of $\operatorname{Aut}_{p} X^{*}$ corresponds an ideal $\mathcal{I}_{p}(G)$ in the algebra $\mathcal{P}_{*}$ of continuous functions $\operatorname{Aut}_{p} X^{*} \rightarrow \mathbb{F}_{p}$. Such ideals behave better than in the case of Aut $X^{*}$, in that they always have "nice generating sets". At the end of the chapter, we give some conditions on $\mathcal{I}_{p}(G)$ for $G$ to be torsion-free, or for the spinal subgroup of $G$ to be torsion.

Many examples of self-similar groups are discrete subgroups of $\operatorname{Aut}_{p} X^{*}$. In Appendix $\mathrm{A}$ we give generators for the annihilator of several groups, including the well-known examples of Grigorchuk and of Gupta and Sidki.

\section{Five Directions}

Chapter 3 is almost independent from the rest of this work, and is the beginning of a possible new development of the topic, relating to algebraic geometry. Modern algebraic geometry contains the whole of commutative algebra. Indeed, to any algebra (commutative, with unit), there is a corresponding affine scheme. Within this correspondence, Hopf algebras are associated to affine group schemes. We briefly recall the relevant definitions, and introduce the wreath product of affine 
group schemes. We prove that the constant scheme associated to Aut $X^{n}$ can be seen as the iterated wreath product of the constant scheme associated to $\operatorname{Sym}(X)$. This is satisfactory, and perhaps not very surprising. More interesting is the case of the infinitesimal scheme $\boldsymbol{\alpha}_{p}$, the "Frobenius kernel". Although this scheme has only one geometric point on fields, we shall see that the Lie algebra of the iterated wreath product of $\boldsymbol{\alpha}_{p}$ is isomorphic to the Lie algebra obtained from the lower central series of $\operatorname{Aut}_{p} X^{n}$.

Building on previous work by Kaloujnine [Kal95], Rozhkov [Roz96], Bartholdi and Grigorchuk [BG00,Bar05] and Bartholdi and Sidki [BS06], we investigate in Chapter 4 the ideals associated to the lower central series and automorphism towers of some specific examples. We shall see that in some cases, these series of groups present surprising similarities.

In Chapter 5, we define the Hausdorff dimension of subgroups of Aut $X^{*}$. I investigated this subject some time ago, providing the first explicit examples of groups of irrational dimension in [Sie08]. We relate this to the core chapters. In particular, we give a formula to compute the Hausdorff dimension of $G$ from a nice generating set of $\mathcal{I}_{p}(G)$.

I have considered congruence problems in a joint work with Laurent Bartholdi and Pavel Zalesskii [BSZ09], and Chapter 6 is an addendum to this article. A regular branch group $G$ has three profinite topologies associated to it. The finest is the profinite topology, where a basis of neighbourhoods of the identity consists of all finite-index subgroups of $G$. A coarser topology is the branch topology, where the rigid stabilizers of $G$ form a basis of neighbourhoods of the identity. Still coarser is the congruence topology, defined by the level-stabilizers. Correspondingly, there are three congruence kernels, and we now know that they might all be non-trivial. This is developed in the aforementioned article. In Chapter 6, we describe the structure of the rigid kernel, and provide an example where this kernel is infinite.

Finally, Chapter 7 is devoted to "twisted twins" of the Grigorchuk group G. We consider a group $G_{2}$ which contains all the spinal elements of the closure of $G$. This group $G_{2}$ is still self-similar, regular branch and torsion. We compute a recursive presentation for it. This was the original motivation for the article [BS09] which studies "the" twisted twin of the Grigorchuk group.

\section{Acknowledgements}

I express deep gratitude to Professor Laurent Bartholdi, whom I freely attacked with numerous questions during four years. He has always offered an attentive ear to my questions, and I particularly appreciated his great knowledge and true honesty. In a sense, I owe him a vision of "open-source" mathematics. I am also grateful to him for careful reading of previous versions of this manuscript.

Among many other professors who influenced me during the preparation of this work, I am especially grateful to Professors Said Sidki and Pavel Zalesskii. Both in Switzerland and Brazil, I deeply appreciated enlightening discussions, collaboration, and many good times. 
This work has been supported by the Swiss National Science Foundation, the Ecole Polytechnique Fédérale de Lausanne and the Eidgenössische Technische Hochschule Zürich. I acknowledge all three institutions. In particular, I warmly thank Professors Eva Bayer and Marc Burger who helped me moving from Lausanne to Zürich.

A lot of thanks go to Mesdames Spillmann, Moinon, Malzacher-Leinhard, Maurer and Dingenotto, without whom I would not have been able to deliver this document. They did an extraordinary job, getting me out of the most confuse situations.

Many thanks go to all the people who bore my presence for several hours in the same room, in particular to Floriane Pochon, Emmanuel Lequeu, Hartwig Mayer and René Birkner. I enjoyed a lot your company and owe you many good memories.

I am very grateful to Bastien Faller and Rachel Bendjama, Stéphane Félix, René Hartung and Hadi Hedayatzadeh. Inspiring discussions with these people contributed to several parts of this thesis. Also, I would like to thank all the other people with whom I shared mathematical discussions, here and abroad.

Thanks to all my friends, thanks to my family and special thanks to Colombe, for your great care, even after suffering the whole construction of this work, close to me. 


\section{Chapter 1}

\section{Foundations}

We settle the notation and give the definitions which are relevant for the whole of this work.

Sections 1.1 to 1.7 form the core of the chapter. We start with the definition of automorphisms of rooted trees and go all the way to the ideals associated to subgroups, also introducing the key notions of self-similarity and branching. Sections 1.8 and 1.9 focus on the case of regular branch groups, and contain some first results which are easy to formulate within our setting. After this we state a few questions which naturally showed up. There is a table on Page 21 which contains a summary of all the definitions needed.

This work is a journey between the worlds of self-similar groups and of profinite groups. Excellent references are [Nek05] for self-similar groups, and [RZ00] or [Wil98] for profinite groups.

\section{General Conventions}

We adopt the following conventions:

- If a group $G$ acts on a set $X$, we denote by $x^{g}$ the image of $x \in X$ under the action of $g \in G$.

- When $g$ and $h$ are elements of the same group, we write $g^{h}=h^{-1} g h$ and $[g, h]=g^{-1} h^{-1} g h$.

- The algebras we consider are all commutative, with unit.

\subsection{Definition of Aut $X^{*}$ and $\mathcal{A}_{*}$}

We give the main definitions and properties concerning two objects: Aut $X^{*}$ and $\mathcal{A}_{*}$. These are dual to one another, and they play a central role in the whole of this work.

We let $X$ be a finite set and $k$ be a finite field. An important special case shall be the situation $X=k$, but we do not assume this in general. We let Aut $X^{*}$ denote the automorphism group of the regular $|X|$-ary rooted tree, and we let 


\subsection{Definition of Aut $X^{*}$ and $\mathcal{A}_{*}$}

$\mathcal{A}_{*}$ be the algebra of continuous functions Aut $X^{*} \rightarrow k$. To understand precisely what this means, we shall detail these definitions.

Let $X^{*}$ be the free monoid on $X$. As a set, we identify $X^{*}$ with the vertices of a regular $|X|$-ary rooted tree. Let $X^{n}$ be the set of words of length $n$ in $X$. This corresponds to the $n$-th level of the tree. $X^{*}$ is graded, in the sense that $X^{*}=\bigcup X^{n}$, and $X^{m} X^{n}=X^{m+n}$. The depth or level of a vertex $v \in X^{*}$, denoted by $|v|$, is the length of $v$ as a word in $X$. We shall refer to the operation of $X^{*}$ as multiplication or concatenation, and denote by $u * v=u v$ the concatenation of $u$ and $v$. Multiplication by $v$ is a map of degree $|v|$ on $X^{*}$.

A word $u$ is a prefix of $v$ if there is $w$ so that $u w=v$; in this case we shall write $u \leq v$. This defines a partial order on $X^{*}$. In the tree picture, this corresponds to $u$ being an ancestor of $v$. Consider two integers $m \geq n \geq 0$. To each $u \in X^{m}$ there is a unique $v \in X^{n}$ satisfying $v \leq u$. Thus there is a unique map Prefix $x_{n, m}: X^{m} \rightarrow X^{n}$ which sends $u$ to $v$ in this way. The sets $X^{n}$, together with these maps, form an inverse system of sets, and the profinite set $X^{\omega}=\lim X^{n}$ is called the boundary of $X^{*}$. It can be identified with the set of right-infinite sequences over $X$. The partial order $\leq$ can be consistently extended to $X^{*} \cup X^{\omega}$. For $S \subseteq X^{*} \cup X^{\omega}$, we define $\operatorname{Prefix}(S)$ as the set of $v \in X^{*}$ so that $v \leq w$ holds for some $w$ in $S$.

An automorphism $g$ of $X^{*}$ is defined as a permutation of $X^{*}$ which preserves the partial order. This is the same as a graph automorphism of the $|X|$-ary rooted tree. Such a permutation necessarily preserves the depth of vertices. Thus it induces a permutation on the $n$-th level $X^{n}$ of the tree for all $n \geq 0$. We let $\operatorname{Stab}(n)$ be the kernel of the action of Aut $X^{*}$ on $X^{n}$. We call this group the $n$-th level stabilizer. The quotient Aut $X^{n}=\operatorname{Aut} X^{*} / \operatorname{Stab}(n)$ is the automorphism group of a finite $|X|$-ary tree with $n$ levels.

Via the map $X^{m} \rightarrow X^{n}$, an automorphism $g \in$ Aut $X^{m}$ induces an automorphism $\pi_{n, m}(g) \in$ Aut $X^{n}$ for all $0 \leq n \leq m$. The maps $\pi_{n, m}$ : Aut $X^{m} \rightarrow$ Aut $X^{n}$ form an inverse system of groups, and the inverse limit of this system is Aut $X^{*}=\lim _{\text {Aut }} X^{n}$, together with the natural maps $\pi_{n}:$ Aut $X^{*} \rightarrow$ Aut $X^{n}$. Thus Aut $X^{*}$ is a profinite group, and $\{\operatorname{Stab}(n) \mid n \geq 0\}$ is a basis of neighbourhoods of the identity in Aut $X^{*}$.

We endow Aut $X^{n}$ and the finite field $k$ with the discrete topology. We let $\mathcal{A}_{n}$ be the set of continuous maps Aut $X^{n} \rightarrow k$. Since the topology is discrete on Aut $X^{n}$, this is just the set of all maps Aut $X^{n} \rightarrow k$. Better than a set, $\mathcal{A}_{n}$ has a natural $k$-algebra structure. The operations are defined pointwise, using the algebra structure of $k$. The algebra $\mathcal{A}_{n}$ even inherits a Hopf algebra structure from the group structure of Aut $X^{n}$, but we shall not need this fact here.

For $m \geq n$, the map $\pi_{n, m}$ : Aut $X^{m} \rightarrow$ Aut $X^{n}$ induces an injection $\iota_{m, n}$ : $\mathcal{A}_{n} \rightarrow \mathcal{A}_{m}$ by

$$
\left(\iota_{m, n} f\right)(g)=f\left(\pi_{n, m} g\right) .
$$

The maps $\iota_{m, n}: \mathcal{A}_{n} \rightarrow \mathcal{A}_{m}$ form a direct system of algebras, and the direct limit of this system is $\mathcal{A}_{*}=\bigcup \mathcal{A}_{n}$, together with the natural maps $\mathcal{A}_{n} \subseteq \mathcal{A}_{*}$. It is clear that $\mathcal{A}_{*}$ is the set of continuous maps Aut $X^{*} \rightarrow k$, since any such map factors through some Aut $X^{n}$ (see [RZ00, Lemma 1.1.16]). 
It follows from this definition that $\mathcal{A}_{*}$ is a filtered algebra. As a consequence, $\mathcal{A}_{*}$ is infinite-dimensional, and locally finite-dimensional. The depth of an element $f \in \mathcal{A}_{*}$ is defined as the least integer $n$ so that $f$ is in $\mathcal{A}_{n}$.

\subsection{Zariski Topology}

Consider a subset $S$ of Aut $X^{*}$. We write $\mathcal{I}(S) \subseteq \mathcal{A}_{*}$ for the annihilator of $S$, i.e. the ideal of functions vanishing on $S$. If $I$ is a subset of $\mathcal{A}_{*}$, we let $\mathcal{V}(I)$ be the annihilator of $I$, i.e. the set of $g \in \operatorname{Aut} X^{*}$ so that $f(g)=0$ for all $f \in I$.

We call a subset $S \subseteq$ Aut $X^{*}$ Zariski-closed if there is $I \subseteq \mathcal{A}_{*}$ so that $S=$ $\mathcal{V}(I)$. The relations

$$
\mathcal{V}\left(I_{1} \cap I_{2}\right)=\mathcal{V}\left(I_{1}\right) \cup \mathcal{V}\left(I_{2}\right), \quad \mathcal{V}\left(\sum_{\alpha} I_{\alpha}\right)=\bigcap_{\alpha} \mathcal{V}\left(I_{\alpha}\right)
$$

hold for all ideals $I_{\alpha}$ of $\mathcal{A}_{*}$, and we have $\mathcal{V}(0)=$ Aut $X^{*}$ and $\mathcal{V}(1)=\varnothing$ (see [Bou61, II, $\left.\left.\S 4, n^{\circ} 3\right]\right)$. Therefore the Zariski-closed sets form the closed sets of a topology, the Zariski topology. In fact this topology coincides with the topology of Aut $X^{*}$ :

Lemma 1.2.1. The Zariski and the profinite topology of Aut $X^{*}$ coincide.

Proof. Consider an ideal $I \subseteq \mathcal{A}_{*}$, and define $I_{n}=I \cap \mathcal{A}_{n}$. Write $S_{n}=\mathcal{V}\left(I_{n}\right)$; this is a closed subset of Aut $X^{*}$. Then $\mathcal{V}(I)$ is equal to $\bigcap S_{n}$, a closed subset of Aut $X^{*}$.

Conversely, if $S \subseteq$ Aut $X^{*}$ is closed, then $S$ is the inverse limit of the sets $S_{n}=\pi_{n}(S)$. Let $I_{n} \subseteq \mathcal{A}_{n}$ be the ideal associated to $S_{n}$ through $I_{n}=\mathcal{I}\left(\pi_{n}^{-1}\left(S_{n}\right)\right)$. Then $\mathcal{V}\left(I_{n}\right)$ is Zariski-closed and so is $S=\bigcap \mathcal{V}\left(I_{n}\right)$.

Recall an ideal $I$ is radical if whenever $f^{n}$ is in $I$ for some $n>0$, then $f$ also belongs to $I$.

Lemma 1.2.2. All ideals of $\mathcal{A}_{*}$ are radical.

Proof. Let $q$ be the cardinality of $k$. The identities $Z^{q^{m}}=Z$ hold in $k$ for all $m \geq 0$. Therefore they also hold in $\mathcal{A}_{*}$. As a consequence, if $f^{n}$ is in the ideal $I$ for some $n>0$, then $f^{q^{m}}$ is in $I$ for some $m \geq 0$, and therefore $f$ belongs to $I$. Thus $I$ is radical.

Using the previous lemma, we can rephrase Proposition 11 of [Bou61, II, §4]. This yields

Proposition 1.2.3. Let $I$ be a subset of $\mathcal{A}_{*}$ and $S$ a subset of Aut $X^{*}$.

(i) $\mathcal{V}(I)$ is closed in Aut $X^{*}$ and $\mathcal{I}(S)$ is an ideal of $\mathcal{A}_{*}$.

(ii) $\mathcal{I}(\mathcal{V}(I))$ is the ideal generated by $I$, and $\mathcal{V}(\mathcal{I}(S))$ is the closure of $S$.

(iii) The maps $\mathcal{I}$ and $\mathcal{V}$ define order-reversing bijections which are inverse to one another, between the closed subsets of Aut $X^{*}$ and the ideals of $\mathcal{A}_{*}$. 


\subsection{Actions of $X^{*}$}

\subsection{Actions of $X^{*}$}

Consider an automorphism $g$ of $X^{*}$, and let $v \in X^{*}$ be any vertex. The commutative diagram

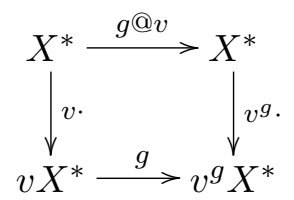

defines the symbol $g @ v$ : the state of $g$ at $v$. A similar diagram defines $\bullet @ v$ : Aut $X^{n} \rightarrow$ Aut $X^{n-|v|}$ for $n \geq|v|$.

Lemma 1.3.1. The above defines a right action of $X^{*}$ on Aut $X^{*}$ : the relations

$$
(g @ u) @ v=g @ u v, \quad g @ \varnothing=g
$$

hold. Moreover, • @ $v$ is not a group homomorphism, but satisfies the cocycle relation

$$
(g h) @ v=(g @ v)\left(h @ v^{g}\right) .
$$

Proof. The relation $g @ \varnothing=g$ is obvious from the definition. The commutative diagrams
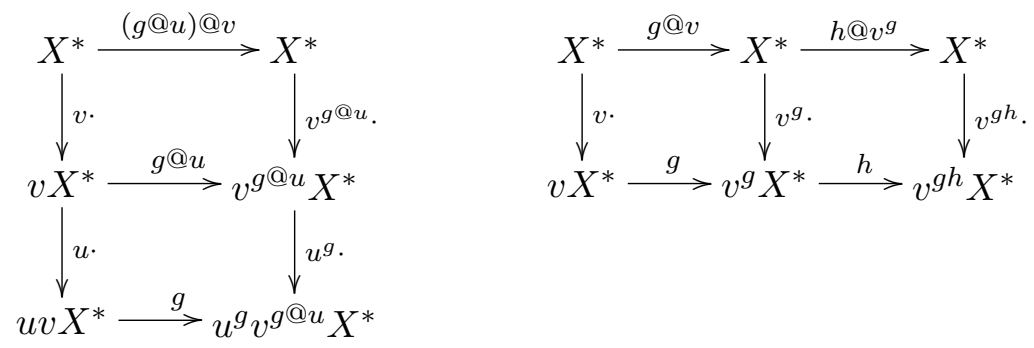

prove the other claims, since $(u v)^{g}=u^{g} v^{g @ u}$ holds by definition.

Consider the commutative diagram

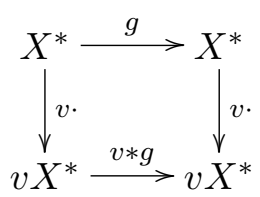

This defines the automorphism $v * g$ of $v X^{*}$. We extend $v * g$ to an automorphism of $X^{*}$ by imposing $v * g$ to fix every vertex which is in the complement of $v X^{*}$. Similarly we define $v * \bullet$ : Aut $X^{n} \rightarrow$ Aut $X^{n+|v|}$ for $n \geq 0$.

Lemma 1.3.2. The above defines a left action of $X^{*}$ on Aut $X^{*}$ : the relations

$$
u *(v * g)=(u v) * g, \quad \quad \varnothing * g=g
$$

hold. Moreover, $v * \bullet$ is a group homomorphism. 
Proof. Similar to Lemma 1.3.1.

The two actions of $X^{*}$ on Aut $X^{*}$ are related by

$$
(v * g) @ v=g .
$$

On the other hand $v *(g @ v)$ is different from $g$, in general.

Consider a (not necessarily continuous) function $f:$ Aut $X^{*} \rightarrow k$. We define the $v$-translate of $f$ and the state of $f$ at $v$ as follows:

$$
(v * f)(g)=f(g @ v) \quad(f @ v)(g)=f(v * g)
$$

for all $v \in X^{*}$ and $g \in$ Aut $X^{*}$. Clearly, $v * \bullet$ and $\bullet @ v$ are algebra endomorphisms. Moreover the relation

$$
(v * f) @ v=f
$$

holds for all $v \in X^{*}$.

The above defines a left and a right action of $X^{*}$ on $\mathcal{A}_{*}$. It is clear that $v * \bullet$ is a map of degree $|v|$, and $\bullet @ v$ is a map of degree $-|v|$. Using $k$-linearity on $\mathcal{A}_{*}$, we extend these actions to the monoid ring $k\{X\}$ of $X^{*}$. This results in linear maps

$$
\text { @ : } \mathcal{A}_{*} \otimes k\{X\} \rightarrow \mathcal{A}_{*} \quad \text { and } \quad *: k\{X\} \otimes \mathcal{A}_{*} \rightarrow \mathcal{A}_{*} \text {. }
$$

\subsection{Self-Similarity and Branching}

We define the notions of self-similarity and branching, which are dual to one another under the duality Aut $X^{*} \leftrightarrow \mathcal{A}_{*}$ (see Proposition 1.4.2).

Consider a subset $S$ of Aut $X^{*}$ or $\mathcal{A}_{*}$. We call $S$

- self-similar if $S @ v \subseteq S$ for all $v \in X^{*}$;

- branching if $v * S \subseteq S$ for all $v \in X^{*}$.

Clearly, Aut $X^{*}$ and $\mathcal{A}_{*}$ are both self-similar and branching. Appendix $\mathrm{A}$ contains some examples of finitely generated subgroups of Aut $X^{*}$ which are self-similar and/or branching.

Let $S$ be a subset of Aut $X^{*}$ (resp. of $\mathcal{A}_{*}$ ). We let $S @ v^{-1}$ be the set of $s \in$ Aut $X^{*}$ (resp. $s \in \mathcal{A}_{*}$ ) so that $s @ v$ is in $S$. We let $v^{-1} * S$ be the set of $s \in$ Aut $X^{*}$ (resp. $s \in \mathcal{A}_{*}$ ) so that $v * s$ is in $S$. Note the following straightforward consequences which hold for all $S \subseteq$ Aut $X^{*}$ and all $S \subseteq \mathcal{A}_{*}$ :

$$
(v * S) @ v=S, \quad\left(S @ v^{-1}\right) @ v=S, \quad v^{-1} *(v * S)=S, \quad v^{-1} *\left(S @ v^{-1}\right)=S .
$$

Let $S$ be a subset of Aut $X^{*}$ or $\mathcal{A}_{*}$.

- The self-similar closure of $S$ is $S @ X^{*}=\bigcup_{v \in X^{*}} S @ v$, the smallest selfsimilar set containing $S$. 


\subsection{Self-Similarity and Branching}

- The self-similar core of $S$ is $S @\left(X^{*}\right)^{-1}=\bigcap_{v \in X^{*}} S @ v^{-1}$, the biggest self-similar set contained in $S$.

- The branching closure of $S$ is $X^{*} * S=\bigcup_{v \in X^{*}} v * S$, the smallest branching set containing $S$.

- The branching core of $S$ is $\left(X^{*}\right)^{-1} * S=\bigcap_{v \in X^{*}} v^{-1} * S$, the biggest branching set contained in $S$.

It is easy to see that if $G \leq$ Aut $X^{*}$ is a group, then so is $\left(X^{*}\right)^{-1} * G$. Also, the set $S @\left(X^{*}\right)^{-1}$ can be seen as the set of elements which satisfy the "patterns" in $S$, in a more general sense than what is described in [Gri05, Section 7]. More precisely, let $S$ be a set of "legal" elements. The set $S @\left(X^{*}\right)^{-1}$ is the set of automorphisms $g \in$ Aut $X^{*}$ so that any state $g @ v$ is legal: $S @\left(X^{*}\right)^{-1}=\{g \in$ Aut $X^{*} \mid g @ v \in S$ for all $\left.v \in X^{*}\right\}$.

Proposition 1.4.1. For all subsets $S$ of Aut $X^{*}$ and of $\mathcal{A}_{*}$, we have the following equivalences

$$
\begin{aligned}
S \text { is self-similar } & \Longleftrightarrow S @ X^{*} \subseteq S \Longleftrightarrow S \subseteq S @\left(X^{*}\right)^{-1}, \\
S \text { is branching } & \Longleftrightarrow X^{*} * S \subseteq S \Longleftrightarrow S \subseteq\left(X^{*}\right)^{-1} * S .
\end{aligned}
$$

Proof. For each line, the first equivalence holds by definition, and the second is straightforward.

Proposition 1.4.2. Let $S$ be a closed subset of Aut $X^{*}$. Then

- $S$ is self-similar if and only if $\mathcal{I}(S)$ is branching;

- $S$ is branching if and only if $\mathcal{I}(S)$ is self-similar.

This is essentially a consequence of Equation (1.3.1). We shall prove some related results before coming back to the proof of this proposition.

Lemma 1.4.3. Consider subsets $S \subseteq$ Aut $X^{*}$ and $I \subseteq \mathcal{A}_{*}$ and let $v \in X^{*}$ be a vertex. We have

(i) $v^{-1} * \mathcal{I}(S)=\mathcal{I}(S @ v)$;

(ii) $\mathcal{I}(S) @ v^{-1}=\mathcal{I}(v * S)$;

(iii) $v^{-1} * \mathcal{V}(S)=\mathcal{V}(S @ v)$;

(iv) $\mathcal{V}(S) @ v^{-1}=\mathcal{V}(v * S)$.

Proof. (i) follows from the equivalences

$$
\begin{aligned}
f \in \mathcal{I}(S @ v) & \Longleftrightarrow f(s @ v)=0 \text { for all } s \in S \Longleftrightarrow(v * f)(s)=0 \text { for all } s \in S \\
& \Longleftrightarrow v * f \in \mathcal{I}(S) \Longleftrightarrow f \in v^{-1} * \mathcal{I}(S) .
\end{aligned}
$$

The other relations are proved in the same way. 
Corollary 1.4.4. Let $S$ be a closed subset of Aut $X^{*}$ and write $I=\mathcal{I}(S)$. Then

$$
\left(X^{*}\right)^{-1} * S=\mathcal{V}\left(I @ X^{*}\right), \quad S @\left(X^{*}\right)^{-1}=\mathcal{V}\left(X^{*} * I\right) .
$$

Proof. These are consequences of Lemma 1.4 .3 and of the relations (1.2.1).

Corollary 1.4.5. Let $G \leq$ Aut $X^{*}$ be closed group. The annihilator of the maximal branching subgroup of $G$ is the ideal generated by $X^{*} @ \mathcal{I}(G)$.

Proof. By definition the maximal branching subgroup of $G$ is $\left(X^{*}\right)^{-1} * G$. By the previous corollary, this is equal to $\mathcal{V}\left(\mathcal{I}(G) @ X^{*}\right)$, and Proposition 1.2 .3 yields the result.

Proof of Proposition 1.4.2. Write $I=\mathcal{I}(S)$. Since $S$ is closed we have $S=\mathcal{V}(I)$. We use Corollary 1.4.4 and Proposition 1.4.1.

Suppose $S$ is self-similar, so that $S \subseteq S @\left(X^{*}\right)^{-1}=\mathcal{V}\left(X^{*} * I\right)$. Applying $\mathcal{I}$ we get $J \subseteq I$, where $J$ is the ideal generated by $X^{*} * I$. Thus in particular $I$ contains $X^{*} * I$, and therefore $I$ is branching. Conversely if $I$ is branching, then $X^{*} * I \subseteq I$, and so applying $\mathcal{V}$ we get $S \subseteq S @\left(X^{*}\right)^{-1}$, and so $S$ is self-similar.

The second assertion follows exactly in the same way.

We shall need the following corollaries in Section 1.8:

Corollary 1.4.6. Consider $S=\mathcal{V}(I)$ with $I \subseteq \mathcal{A}_{n}$. Then $g \in$ Aut $X^{*}$ is in $\mathcal{V}\left(X^{*} * I\right)$ if and only if $\pi_{n}(g @ v)$ is in $\pi_{n}(S)$ for all $v \in X^{*}$.

Proof. By Corollary 1.4.4, we have $\mathcal{V}\left(X^{*} * I\right)=S @\left(X^{*}\right)^{-1}$. The latter is just $\left\{g \in\right.$ Aut $X^{*} \mid g @ v \in S$ for all $\left.v \in X^{*}\right\}$ by definition. Since $I$ is a subset of $\mathcal{A}_{n}$, it is clear that $g$ is in $S$ if and only if $\pi_{n}(g)$ is in $\pi_{n}(S)$. Putting this together yields the corollary.

Corollary 1.4.7. Consider $S=\mathcal{V}\left(X^{*} * I\right)$ with $I \subseteq \mathcal{A}_{*}$. Then $\left(X^{*}\right)^{-1} * S=$ $\mathcal{V}\left(\left(I @ X^{*}\right)+\left(X^{*} * I\right)\right)$.

Proof. By the proposition, we have $\left(X^{*}\right)^{-1} * S=\mathcal{V}\left(\mathcal{I}(S) @ X^{*}\right)$. Now $\mathcal{I}(S)$ is the ideal generated by $X^{*} * I$, and so we need to prove

$$
\left(\left(X^{*} * I\right) @ X^{*}\right)=\left(I @ X^{*}\right)+\left(X^{*} * I\right) .
$$

This follows from the formal computation $\left(\left(X^{*} * I\right) @ X^{*}\right)=\sum_{u, v \in X^{*}}(u * I) @ v=$ $\sum_{v \in X^{*}}(I @ v)+\sum_{v \in X^{*}}(v * I)$, where we used Equation (1.3.2).

\subsection{Decompositions and Generators of Aut $X^{*}$ and $\mathcal{A}_{*}$}

The group Aut $X^{n}$ is isomorphic to the $n$-fold wreath power of $\operatorname{Sym}(X)$ :

$$
\text { Aut } X^{n} \simeq \operatorname{Sym}(X) \prec \cdots \prec \operatorname{Sym}(X) \text {. }
$$




\subsection{Decompositions and Generators of Aut $X^{*}$ and $\mathcal{A}_{*}$}

Therefore we have the group isomorphism Aut $X^{m+n} \simeq \operatorname{Aut} X^{m}$ 々 Aut $X^{n}$ which in the limit becomes

$$
\psi_{n}: \text { Aut } X^{*} \stackrel{\sim}{\longrightarrow} \text { Aut } X^{*} \imath \text { Aut } X^{n}
$$

The map $\psi_{n}$ is even a homeomorphism, when the target is endowed with the product topology. We define the section

$$
\sigma_{n}: \text { Aut } X^{n} \rightarrow \operatorname{Aut} X^{*}
$$

of $\pi_{n}$ using the decomposition $\psi_{n}$. Namely, we require the image of $\sigma_{n}$ to be the "top group" 1 々 Aut $X^{n}$. More precisely, we impose $\sigma_{n}(g) @ v=1$ for all $v \in X^{*},|v| \geq n$. The map $\sigma_{n}$ is then uniquely determined by the relation $\pi_{n} \circ \sigma_{n}=\operatorname{id}_{\text {Aut } X^{n}}$.

Dually, we have the algebra isomorphisms

$$
\mathcal{A}_{n} \simeq \bigotimes_{0 \leq|v|<n} v * \mathcal{A}_{1}
$$

and

$$
\mathcal{A}_{*} \simeq \mathcal{A}_{*}^{\otimes\left|X^{n}\right|} \otimes \mathcal{A}_{n}, \quad \mathcal{A}_{m+n} \simeq \mathcal{A}_{m}^{\otimes\left|X^{n}\right|} \otimes \mathcal{A}_{n}
$$

The section $\sigma_{n}$ induces a projection

$$
\varpi_{n}: \mathcal{A}_{*} \rightarrow \mathcal{A}_{n}
$$

defined by $\varpi_{n}(f)(g)=f\left(\sigma_{n}(g)\right)$.

As we do in the next lemma, we shall often identify the group $\operatorname{Sym}(X)$ with its image under $\sigma_{1}$.

Lemma 1.5.1. The group Aut $X^{n}$ is generated by $\left\{v * \sigma\left|v \in X^{*}, 0 \leq\right| v \mid<\right.$ $n, \sigma \in \operatorname{Sym}(X)\}$.

Proof. Straightforward.

Corollary 1.5.2. The group Aut $X^{*}$ is topologically generated by $\{v * \sigma \mid v \in$ $\left.X^{*}, \sigma \in \operatorname{Sym}(X)\right\}$.

Corollary 1.5.3. The group Aut $X^{*}$ is topologically generated by $\operatorname{Sym}(X)$ as a branching group.

The generating set of Corollary 1.5 .2 is certainly not minimal. For a smaller generating set, one can take for example $\left\{x^{n} * \sigma \mid n \geq 0, \sigma \in S\right\}$, with $x$ any element of $X$ and $S$ any generating set of $\operatorname{Sym}(X)$.

Let $\chi_{\sigma}: \operatorname{Sym}(X) \rightarrow k$ be the characteristic function of $\sigma \in \operatorname{Sym}(X)$.

Lemma 1.5.4. The algebra $\mathcal{A}_{n}$ is generated by $\left\{v * \chi_{\sigma}\left|v \in X^{*}, 0 \leq\right| v \mid<n, \sigma \in\right.$ $\operatorname{Sym}(X)\}$. 
Proof. We proceed by induction. Since Aut $X^{n}$ is a finite group, it is enough to show that the characteristic function $\chi_{g}$ of each element $g \in$ Aut $X^{n}$ is a sum of products of $v * \chi_{\sigma}$.

For $n=0$, the group Aut $X^{n}$ is trivial, and thus 1 is a characteristic function on the only one element. Suppose we proved the claim up to $n$, so that for each element $g \in$ Aut $X^{n}$, we know how to express $\chi_{g}$ as a sum of products of $v * \chi_{\sigma}$ with $v$ of length $<n$. We use the isomorphism Aut $X^{n+1}=\operatorname{Aut} X^{n}(\operatorname{Sym}(X)$. As a set, Aut $X^{n+1}$ is just $\left(\prod_{x \in X} x * \operatorname{Aut} X^{n}\right) \times \operatorname{Sym}(X)$. Take an element $g \in$ Aut $X^{n+1}$ and decompose it as $g=\left(\prod_{x \in X} x *(g @ x)\right) \tau$ under the above bijection. Then $\chi_{g}=\left(\prod_{x \in X} x *\left(\chi_{g} @ x\right)\right) \chi_{\tau}$ expresses $\chi_{g}$ as a sum of products of $v * \chi_{\sigma}$.

Corollary 1.5.5. The algebra $\mathcal{A}_{*}$ is generated by $\left\{v * \chi_{\sigma} \mid v \in X^{*}, \sigma \in \operatorname{Sym}(X)\right\}$.

Corollary 1.5.6. The algebra $\mathcal{A}_{*}$ is generated by $\left\{\chi_{\sigma} \mid \sigma \in \operatorname{Sym}(X)\right\}$ as a branching algebra.

\subsection{The Permutation Modules $k\{X\}$ and $k\{\{X\}\}$}

Let $G$ be a closed subgroup of Aut $X^{*}$. The linear span $k\{X\}_{n}$ of $X^{n}$ is a finite $G$-module, and the action of $G$ is continuous. Letting $G$ act on the right on $X^{n}$, it follows that $k\{X\}_{n}$ is a right $G$-module. We write $k\{X\}_{n}^{\circ}$ for the dual module of $k\{X\}_{n}$. The module $k\{X\}_{n}^{\circ}$ is isomorphic to $k\{X\}_{n}$ as a vector space, and is canonically a left $G$-module. Clearly, $\left(k\{X\}_{n}^{\circ}\right)^{\circ}$ is canonically isomorphic to $k\{X\}_{n}$, and we shall consider the elements of $k\{X\}_{n}$ as functionals on $k\{X\}_{n}^{\circ}$. The evaluation of a functional $f \in k\{X\}_{n}$ on an element $m \in k\{X\}_{n}^{\circ}$ shall be denoted by $\langle f \mid m\rangle$. By definition, the action of $G$ satisfies

$$
\left\langle f^{g} \mid m\right\rangle=\left\langle f \mid m^{g^{-1}}\right\rangle .
$$

If $M$ is a submodule of $k\{X\}_{n}^{\circ}$, the orthogonal of $M$ is the submodule $M^{\perp}$ of $k\{X\}_{n}$ consisting of the elements $f$ with $\langle f \mid m\rangle=0$ for all $m \in M$.

We settle the notation

$$
\begin{aligned}
k\{X\}_{<n} & =\bigoplus_{i=0}^{n-1} k\{X\}_{i}, & k\{X\}_{<n}^{\circ} & =\prod_{i=0}^{n-1} k\{X\}_{i}^{\circ}, \\
k\{X\} & =\bigoplus_{i \geq 0} k\{X\}_{i}, & k\{\{X\}\} & =\prod_{i \geq 0} k\{X\}_{i}^{\circ} .
\end{aligned}
$$

$k\{X\}$ is isomorphic to the monoid ring of $X^{*}$. For $m \geq n$, the projection $\pi_{n, m}$ : $k\{X\}_{<m}^{\circ} \rightarrow k\{X\}_{<n}^{\circ}$ on the first $n$ factors is a morphism of $G$-modules. The projective limit $\varliminf_{n} k\{X\}_{<n}^{\circ}$ is isomorphic to $k\{\{X\}\}$, turning it into a profinite $G$-module. This module is equipped with natural maps

$$
\pi_{n}: k\{\{X\}\} \rightarrow k\{X\}_{<n}^{\circ} .
$$

Slightly overloading notation, we define another map $\pi_{n}: k\{X\} \rightarrow k\{X\}_{<n}$ as the projection on the first $n$ factors. 


\subsection{Subgroups of Aut $X^{*}$}

The dual of $k\{\{X\}\}$ is much bigger than $k\{X\}$. However, $k\{X\}$ is canonically identified with the module of continuous linear functionals $k\{\{X\}\} \rightarrow k$. If $M$ is a subset of $k\{\{X\}\}$, we define the orthogonal of $M$ as the submodule $M^{\perp}$ of $k\{X\}$ consisting of functionals $f$ with $\langle f \mid m\rangle=0$ for all $m \in M$.

The operation of $X^{*}$ can be extended in a unique way to a linear map

$$
*: k\{X\} \otimes k\{X\} \rightarrow k\{X\}
$$

This map is compatible with the grading, in the sense that $k\{X\}_{m} * k\{X\}_{n} \subseteq$ $k\{X\}_{m+n}$.

Similarly, for $u, v \in X^{*}$ we define

$$
u @ v= \begin{cases}w & \text { if } u=v w, \\ 0 & \text { if } v \not \leq u .\end{cases}
$$

Linear extension of this operation yields the linear map

$$
@: k\{X\} \otimes k\{X\} \rightarrow k\{X\} .
$$

This map is compatible with the grading, in the sense that $k\{X\}_{m} @ k\{X\}_{n} \subseteq$ $k\{X\}_{m-n}$.

Dually, the linear maps

$$
*: k\{X\} \otimes k\{\{X\}\} \rightarrow k\{\{X\}\}, \quad @: k\{\{X\}\} \otimes k\{X\} \rightarrow k\{\{X\}\}
$$

are defined via the formulæ

$$
\langle v * f \mid m\rangle=\langle f \mid m @ v\rangle, \quad\langle f @ v \mid m\rangle=\langle f \mid v * m\rangle .
$$

For subsets of $k\{X\}$ and of $k\{\{X\}\}$, self-similarity and branching are defined as in Section 1.4.

\subsection{Subgroups of Aut $X^{*}$}

We define an action

$$
\begin{aligned}
\mathcal{A}_{*} \times \operatorname{Aut} X^{*} & \rightarrow \mathcal{A}_{*} \\
(f, g) & \mapsto f^{g}
\end{aligned}
$$

of Aut $X^{*}$ on $\mathcal{A}_{*}$ by algebra endomorphisms, via the formula

$$
f^{g}(h)=f(g h) .
$$

Here $f$ is a function Aut $X^{n} \rightarrow k$, and the relation defines a new function $f^{g}$ : Aut $X^{n} \rightarrow k$. Thus in particular this action preserves depth of functions.

We note the relations

$$
(v * f)^{g}=v^{g} * f^{g @ v}, \quad \quad(f @ v)^{g}=\left(f^{v * g}\right) @ v .
$$


Proposition 1.7.1. Let $G$ be a closed subset of Aut $X^{*}$ and write $I=\mathcal{I}(G)$. Then $G$ is a group if and only if $I^{g} \subseteq I$ for all $g \in G$.

Proof. The "only if" part is trivial. For the "if", it is enough to show that $G$ is stable under product, because this implies that $\pi_{n}(G)$ is a group for all $n \geq 0$, and thus $G$ is a group. Consider $g, g^{\prime} \in G$ and $f \in I$. Then $f^{g}\left(g^{\prime}\right)=f\left(g g^{\prime}\right)=0$, and thus $g g^{\prime}$ is in $\mathcal{V}(I)$. But $\mathcal{V}(I)$ is equal to $G$, since $G$ is closed.

Proposition 1.7.2. Let $G \leq$ Aut $X^{*}$ be a group generated by a set $S$ and consider a set $I \subseteq \mathcal{A}_{*}$ so that $f(1)=0$ and $f^{s}-f \in \mathcal{I}(G)$ for all $f \in I$ and $s \in S$. Then $I \subseteq \mathcal{I}(G)$.

Proof. The monoid generated by $S$ is dense in $\mathcal{I}(G)$, therefore it is enough to show that any function $f \in I$ vanishes on it. We shall prove by induction on $n$ that $f\left(s_{1} \cdots s_{n}\right)=0$ for all $s_{1}, \ldots, s_{n} \in S$. This holds by assumption for $n=0$. Also, $f\left(s_{1} \cdots s_{n}\right)=f^{s_{1}}\left(s_{2} \cdots s_{n}\right)$ is equal to $f\left(s_{2} \cdots s_{n}\right)$ because $f^{s_{1}} \equiv f(\bmod \mathcal{I}(G))$ and $s_{2} \cdots s_{n}$ is in $G$. Now $f\left(s_{2} \cdots s_{n}\right)$ vanishes by induction hypothesis.

Proposition 1.7.3. Let $G$ be a closed subgroup of Aut $X^{*}$ and consider a function $f \in \mathcal{A}_{*}$. Then $f: G \rightarrow k$ is a group homomorphism if and only if $f^{g} \equiv f+f(g)$ $(\bmod \mathcal{I}(G))$ for all $g \in G$.

Proof. Follows from Equation (1.7.1).

Lemma 1.7.4. Let $G \leq H$ be closed subgroups of Aut $X^{*}$. The following are equivalent:

- $G$ has finite index in $H$;

- There is a finite set $S \subseteq \mathcal{A}_{*}$ so that $G=\mathcal{V}(\mathcal{I}(H)+(S))$.

Proof. If $G$ has index $d$ in $H$, there is $n$ so that $\pi_{n}(G)$ has index $d$ in $\pi_{n}(H)$. Then $S=\mathcal{I}(G) \cap \mathcal{A}_{n}$ is finite and clearly satisfies $G=\mathcal{V}(\mathcal{I}(H)+(S)$ ).

Conversely, if there is such a finite set $S$, then set $n=\max \{\operatorname{depth}(f): f \in S\}$. The index of $\pi_{n}(G)$ in $\pi_{n}(H)$ is finite, and is equal to that of $G$ in $H$ by the choice of $n$.

Lemma 1.7.5. Let $G \leq H$ be closed subgroups of Aut $X^{*}$ and let $n \geq 0$ be an integer. The following are equivalent:

- The indices $[H: G]$ and $\left[\pi_{n}(H): \pi_{n}(G)\right]$ are equal;

- The ideals $\mathcal{I}(G)$ and $\mathcal{I}(H)$ are equal, modulo $\mathcal{A}_{n}$.

Proof. Straightforward. 


\subsection{Regular Branch Groups}

\subsection{Regular Branch Groups}

Let $G$ be a subgroup of Aut $X^{*}$ and let $K=\left(X^{*}\right)^{-1} * G$ be its maximal branching subgroup. Write $X^{n} * K$ for the group generated by $v * K$ with $v \in X^{n}$.

The following three properties are sometimes used to define regular branchness:

(RB-) $G$ is commensurable with $X^{n} * G$ in Aut $X^{*}$ for all $n \geq 0$;

(RB) $X^{n} * K$ has finite index in $G$ for all $n \geq 0$;

$(\mathbf{R B}+) K$ has finite index in the group generated by $G @ X^{*}$.

The implications $(\mathrm{RB}+) \Longrightarrow(\mathrm{RB}) \Longrightarrow(\mathrm{RB}-)$ are straightforward, but the gaps between these properties is not clearly understood, yet. We suspect that all three conditions are equivalent for closed groups.

We shall call a group regular branch if it satisfies the condition (RB) above.

Lemma 1.8.1. Let $G \leq$ Aut $X^{*}$ be a closed, self-similar group which is regular branch over $K$, and consider $n$ so that $[G: K]=\left[\pi_{n}(G): \pi_{n}(K)\right]$. Then $X^{m} *$ $\operatorname{Stab}_{G}(n)=\operatorname{Stab}_{G}(m+n)$ holds for all $m \geq 0$.

Proof. By the choice of $n$, we have $\operatorname{Stab}_{G}(n)=\operatorname{Stab}_{K}(n)$. By self-similarity, we have $\operatorname{Stab}_{G}(m+n) \leq X^{m} * \operatorname{Stab}_{G}(n)=X^{m} * \operatorname{Stab}_{K}(n)$. Due to the fact that $K$ is branching, we also have the inclusion $X^{m} * \operatorname{Stab}_{K}(n) \leq \operatorname{Stab}_{G}(m+n)$, and we deduce the equality $\operatorname{Stab}_{G}(m+n)=X^{m} * \operatorname{Stab}_{G}(n)$.

The following is the analogue of [Šun07, Theorem 3], which characterizes "finitely constrained groups".

Theorem 1.8.2. Let $G$ be a closed, self-similar subgroup of Aut $X^{*}$. Then $G$ is regular branch if and only if $\mathcal{I}(G)$ is finitely generated as a branching ideal.

Proof. Write $K=\left(X^{*}\right)^{-1} * G$ and $I=\mathcal{I}(G)$. By Corollary 1.4.4 the equality $K=\mathcal{V}\left(I @ X^{*}\right)$ holds. Let $S$ be a finite generating set for $I$ as a branching ideal. Then $I @ X^{*}$ is generated by $I$ and $S @ X^{*}$, adding only finitely many new equations to $I$. Therefore $K$ has finite index in $G$ by Lemma 1.7.4. Next, $X^{n} * K \leq \operatorname{Stab}_{G}(n)$ has finite index in $X^{n} * G$, and $\operatorname{Stab}_{G}(n)$ is a subgroup of $X^{n} * G$ by self-similarity. Therefore $X^{n} * K$ has finite index in $G$.

For the converse, suppose $G$ is regular branch. Thus in particular $K$ has finite index in $G$, and there is an integer $d$ so that $\left[\pi_{d-1}(G): \pi_{d-1}(K)\right]=[G: K]$. We claim that $I$ is generated by $I_{d}=I \cap \mathcal{A}_{d}$.

Set $S=\mathcal{V}\left(X^{*} I_{d}\right)$. It is clear that $\pi_{d}(S)=\pi_{d}(G)$ holds, and since $G$ is selfsimilar, we have $G \subseteq S$ by Corollary 1.4.6. We now prove by induction on that $\pi_{n}(S) \subseteq \pi_{n}(G)$ holds for all $n \geq d$. Since $G$ is closed, this will imply $S=G$.

Suppose $\pi_{n}(S) \subseteq \pi_{n}(G)$ holds for some $n \geq d$. Note that $S$ is also self-similar by Proposition 1.4.2, hence $\pi_{n}(S @ x) \subseteq \pi_{n}(G)$ holds for all $x \in X$. Consider $g \in S$ and let $h \in G$ be so that $\pi_{n}(g)=\pi_{n}(h)$. Then $g h^{-1}$ is in $\operatorname{Stab}(n)$, and $\pi_{n}\left(g h^{-1} @ x\right)=\pi_{n}(g @ x) \pi_{n}\left(h^{-1} @ x^{g}\right)$ is in $\pi_{n}(G)$ for all $x \in X$. Therefore $\pi_{n+1}\left(g h^{-1}\right)$ is in $\pi_{n+1}(\operatorname{Stab}(n)) \cap\left(X * \pi_{n}(G)\right)=\pi_{n+1}\left(X * \operatorname{Stab}_{G}(n-1)\right)$. 
By Lemma 1.8.1, $X * \operatorname{Stab}_{G}(n-1)=\operatorname{Stab}_{G}(n)$ holds for all $n \geq d$. We thus have $\pi_{n+1}\left(g h^{-1}\right) \in \pi_{n+1}\left(\operatorname{Stab}_{G}(n)\right)$, whence $\pi_{n+1}(g) \in \pi_{n+1}(G)$. This proves the induction step.

If an ideal $I \subseteq \mathcal{A}_{*}$ is finitely generated as a branching ideal, the depth of $I$ is the depth of a generating set (qua branching ideal) of minimal depth.

Corollary 1.8.3. Let $G \leq$ Aut $X^{*}$ be a closed, self-similar, regular branch group; let $K$ be the maximal branching subgroup of $G$ and let $d$ be the depth of $\mathcal{I}(G)$. Then $d$ is the least integer so that $[G: K]=\left[\pi_{d-1}(G): \pi_{d-1}(K)\right]$.

Proof. If $n$ is big enough so that $[G: K]=\left[\pi_{n-1}(G): \pi_{n-1}(K)\right]$ holds, then in the proof of the theorem we construct a generating set of depth $n$ for $\mathcal{I}(G)$.

Conversely, let $I$ be a generating set of $\mathcal{I}(G)$ of depth $n$. Then $\mathcal{I}(K)=$ $\left(I @ X^{*}\right)+\left(X^{*} * I\right)$ by Corollary 1.4.7, and therefore $\mathcal{I}(G)=\left(X^{*} * I\right)$ is equal to $\mathcal{I}(K)$, modulo $\mathcal{A}_{n-1}$. Lemma 1.7 .5 implies $[G: K]=\left[\pi_{n-1}(G): \pi_{n-1}(K)\right]$.

Corollary 1.8.4. Let $G \leq$ Aut $X^{*}$ be a (not necessarily closed) self-similar group, regular branch over $K$, and suppose $K$ contains $\operatorname{Stab}_{G}(d-1)$. Then $\mathcal{I}(G)$ is generated by $\mathcal{I}(G) \cap \mathcal{A}_{d}$ as a branching ideal.

Proof. The closure $\bar{G}$ of $G$ is self-similar, and it is regular branch over the closure $\bar{K}$ of $K$ because $K$ is a congruence subgroup. Moreover, $[\bar{G}: \bar{K}]=\left[\pi_{d-1}(\bar{G})\right.$ : $\left.\pi_{d-1}(\bar{K})\right]$ holds. Therefore the previous corollary applies.

Write $\Delta^{n}=\sum_{v \in X^{n}} v$.

Proposition 1.8.5. Let $K$ be a closed branching subgroup of Aut $X^{*}$ and let $f \in \mathcal{A}_{*}$ be so that $f: K \rightarrow k$ is a non-zero map. Then $\left\{\Delta^{n} * f \mid n \geq 0\right\}$ is a linearly independent set modulo $\mathcal{I}(K)$.

Proof. Fix a letter $x \in X$ and set $f_{-n}=f @ x^{n}$ and $f_{n}=\Delta^{n} * f$ for $n \geq 0$. Note the relation $f_{n} @ x=f_{n-1}$. Let $d$ be the smallest integer so that $f_{d} \notin \mathcal{I}(K)$. Then $d$ is non-positive because $f: K \rightarrow k$ is non-zero. We prove $\left\{f_{d}, \ldots, f_{n}\right\}$ is linearly independent modulo $\mathcal{I}(K)$. This is true for $n=d$ and we proceed by induction.

Suppose $\left\{f_{d}, \ldots, f_{n}\right\}$ is linearly independent modulo $\mathcal{I}(K)$ and suppose $f^{\prime}=$ $\sum_{i=d}^{n+1} \lambda_{i} f_{i}$ is in $\mathcal{I}(K)$ for some $\lambda_{i} \in k$. Then using Proposition 1.4.2, $\mathcal{I}(K) @ x$ is a subset of $\mathcal{I}(K)$, and therefore $f^{\prime} @ x=\sum_{i=d+1}^{n+1} \lambda_{i} f_{i-1}$ is in $\mathcal{I}(K)$. Using the induction hypothesis, this implies $\lambda_{i}=0$ for all $i \in\{d+1, \ldots, n+1\}$. Therefore $f^{\prime}=\lambda_{d} f_{d}$ and so $\lambda_{d}=0$ due to the choice of $d$.

Let $G$ be a subgroup of Aut $X^{*}$ and consider a function $f \in \mathcal{A}_{*}$. The $G$-depth of $f$ is the least integer $d$ so that $f$ factorizes through $G \rightarrow G / \operatorname{Stab}_{G}(d) \rightarrow k$. In other words $d$ is the only integer so that $f$ is constant on the fibres of $G \rightarrow$ $G / \operatorname{Stab}_{G}(d)$ but not on those of $G \rightarrow G / \operatorname{Stab}_{G}(d-1)$. It is clear that the Aut $X^{*}$-depth is exactly the depth, and that the $G$-depth is always smaller or equal to the depth of a function. 


\subsection{Self-Similarity and Branching Simultaneously}

Theorem 1.8.6. Let $G \leq$ Aut $X^{*}$ be a closed, self-similar, regular branch group; let $d$ be the depth of $\mathcal{I}(G)$ and suppose there exists a continuous group homomorphism $f: G \rightarrow k$ of $G$-depth at least $d$. Then $G$ is not topologically finitely generated.

Proof. Since $f$ is continuous, it factors through some $\pi_{n}$. Because it is a group homomorphism and $\pi_{n}(G)$ is a finite group, $k$ must have positive characteristic.

Let $d^{\prime}$ be the $G$-depth of $f$. By definition, $f$ is not constant on the fibres of $G \rightarrow G / \operatorname{Stab}_{G}\left(d^{\prime}-1\right)$. Since $f$ is a group homomorphism, we deduce that $f$ is not constant on the particular fibre $\operatorname{Stab}_{G}\left(d^{\prime}-1\right)$. On the other hand, $\Delta^{n} * f$ is a group homomorphism $G \rightarrow k$ for all $n \geq 0$ by Lemma 1.8.7. We proceed to prove that these maps are linearly independent modulo $\mathcal{I}(G)$.

By Lemma 1.8.1, $\operatorname{Stab}_{G}\left(d^{\prime}-1\right)$ is a branching subgroup. Proposition 1.8.5 tells us that $\left\{\Delta^{n} * f \mid n \geq 0\right\}$ is linearly independent modulo $\mathcal{I}\left(\operatorname{Stab}_{G}\left(d^{\prime}-1\right)\right)$, and a fortiori modulo $\mathcal{I}(G)$.

This implies that for any $n \geq 0$, the map $\left(f, \ldots, \Delta^{n-1} * f\right)$ is an epimorphism $C_{p}^{n}$, the $n$-th direct power of the cyclic group of order $p$, where $p$ is the characteristic of $k$. This group is $n$-generated, therefore $G$ is at least $n$ generated.

Lemma 1.8.7. Let $G$ be a self-similar subgroup of Aut $X^{*}$ and suppose $f: G \rightarrow k$ is a group homomorphism. Then $\Delta^{n} * f$ is also a group homomorphism $G \rightarrow k$, for all $n \geq 0$.

Proof. The function $\Delta^{n} * f$ is defined on $G$ because $G$ is self-similar. We compute

$$
\begin{aligned}
\left(\Delta^{n} * f\right)(g h) & =\sum_{v \in X^{n}} f((g h) @ v)=\sum_{v \in X^{n}} f\left((g @ v)\left(h @ v^{g}\right)\right) \\
& =\sum_{v \in X^{n}} f(g @ v)+f\left(h @ v^{g}\right)=\left(\Delta^{n} * f\right)(g)+\left(\Delta^{n} * f\right)(h)
\end{aligned}
$$

so $\Delta^{n} * f$ is indeed a group homomorphism. Note that we used self-similarity to ensure $g @ v$ and $h @ v^{g}$ are in $G$.

\subsection{Self-Similarity and Branching Simultaneously}

As a special case of regular branch groups, we investigate which groups are at the same time self-similar and branching.

Consider a subgroup $A$ of $\operatorname{Sym}(X)$. We define $\operatorname{Aut}_{A} X^{*}$ as the subgroup of Aut $X^{*}$ consisting of elements $g$ with $\pi_{1}(g @ v) \in A$ for all $v \in X^{*}$.

Proposition 1.9.1. Let $G$ be a closed, self-similar, branching subgroup of Aut $X^{*}$. Then $G$ is equal to $\operatorname{Aut}_{A} X^{*}$, with $A=\pi_{1}(G)$.

Proof. The inclusion $G \leq \operatorname{Aut}_{A} X^{*}$ holds because $G$ is self-similar. The converse inclusion holds because $G$ is branching and closed.

If $G$ is a profinite group, the derived subgroup of $G$ is $\overline{[G, G]}$, the closure of the group generated by commutators. A group $G$ is perfect if it is equal to its derived subgroup. 
Proposition 1.9.2. The group $\operatorname{Aut}_{A} X^{*}$ is perfect if and only if $A$ is perfect.

Proof. If $A$ is perfect then so is $\operatorname{Aut}_{A} X^{*}$ because it is generated by the groups $v * A$, which are perfect. If $A$ is not perfect then $\operatorname{Aut}_{A} X^{*}$ maps surjectively onto $A=\pi_{1}\left(\operatorname{Aut}_{A} X^{*}\right)$, and further onto $A /[A, A]$, a non-trivial Abelian group. Therefore $\operatorname{Aut}_{A} X^{*}$ is not perfect either.

It is quite surprising that some of the groups $\operatorname{Aut}_{A} X^{*}$ are finitely generated.

Theorem 1.9.3. Let $A$ be a perfect group and suppose one of the following conditions holds:

(i) $\operatorname{Stab}_{A}(x)$ is a perfect group for all $x \in X$, and $A$ is generated by the groups $\left.\operatorname{Stab}_{A}\left(x_{1}\right) \cap \operatorname{Stab}_{A}\left(x_{2}\right)\right)^{\prime}$ with $x_{1} \neq x_{2} ;$

(ii) $A$ is a wreath product $S_{1} \imath \cdots 2 S_{n}$ where each $S_{i}$ acts on a finite set $X_{i}$, and $S_{i}$ is perfect, transitive on $X_{i}$, and distinct points of $X_{i}$ have distinct stabilizers in $S_{i}$.

Then $\operatorname{Aut}_{A} X^{*}$ is finitely generated.

The proof of this theorem relies on two different constructions. For each case a finitely generated, dense subgroup is explicitly given. It is interesting to note that these groups showed up mainly as counterexamples, exhibiting some special properties.

For (i) the construction is due to Neumann [Neu86], and was designed to answer some questions raised in [EP84] concerning just-infinite and "atomic" groups. In [Seg04], Segal constructs groups which have certain prescribed homomorphic images, and groups having very slow, yet superpolynomial, subgroup growth. A special case of Segal's examples is Case (ii). Wilson [Wil04] also used this construction to exhibit the first groups having non-uniform exponential growth. Interestingly, based on the construction of Neumann, Bartholdi [Bar03] constructed a second example of a group of non-uniform exponential growth. The first construction and the case $n=1$ of the second are given, including proofs and written in a language close to ours, in the book [Nek05].

On the other extreme we have

Theorem 1.9.4. Suppose $A$ is not perfect. Then $\operatorname{Aut}_{A} X^{*}$ is not finitely generated.

Proof. $A /[A, A]$ is non-trivial, therefore there is a surjective group homomorphism $f: G \rightarrow k$ with $k$ a finite field. We can thus apply Theorem 1.8.6 with $d=1$.

\subsection{Questions}

Question 1.10.1. Let $G \leq$ Aut $X^{*}$ be a closed self-similar group. Do we have the equivalence $\left(X^{*}\right)^{-1} * G \neq 1 \Longleftrightarrow \operatorname{dim}_{H}(G) \neq 0$ ? Note that the implication $\Longrightarrow$ is clear (see Chapter 5 for the definition of Hausdorff dimension). 
1.10. Questions

An element $g \in$ Aut $X^{*}$ is finite-state if $g @ X^{*}$ is a finite set.

Question 1.10.2. Suppose $K$ is branching and generated by finitely many finitestate elements. Does it imply that $G=K @ X^{*}$ is also finitely generated? Or even that $[G: K]$ is finite?

Question 1.10.3. Does there exist a perfect group $A$ so that $\operatorname{Aut}_{A} X^{*}$ is not finitely generated? 


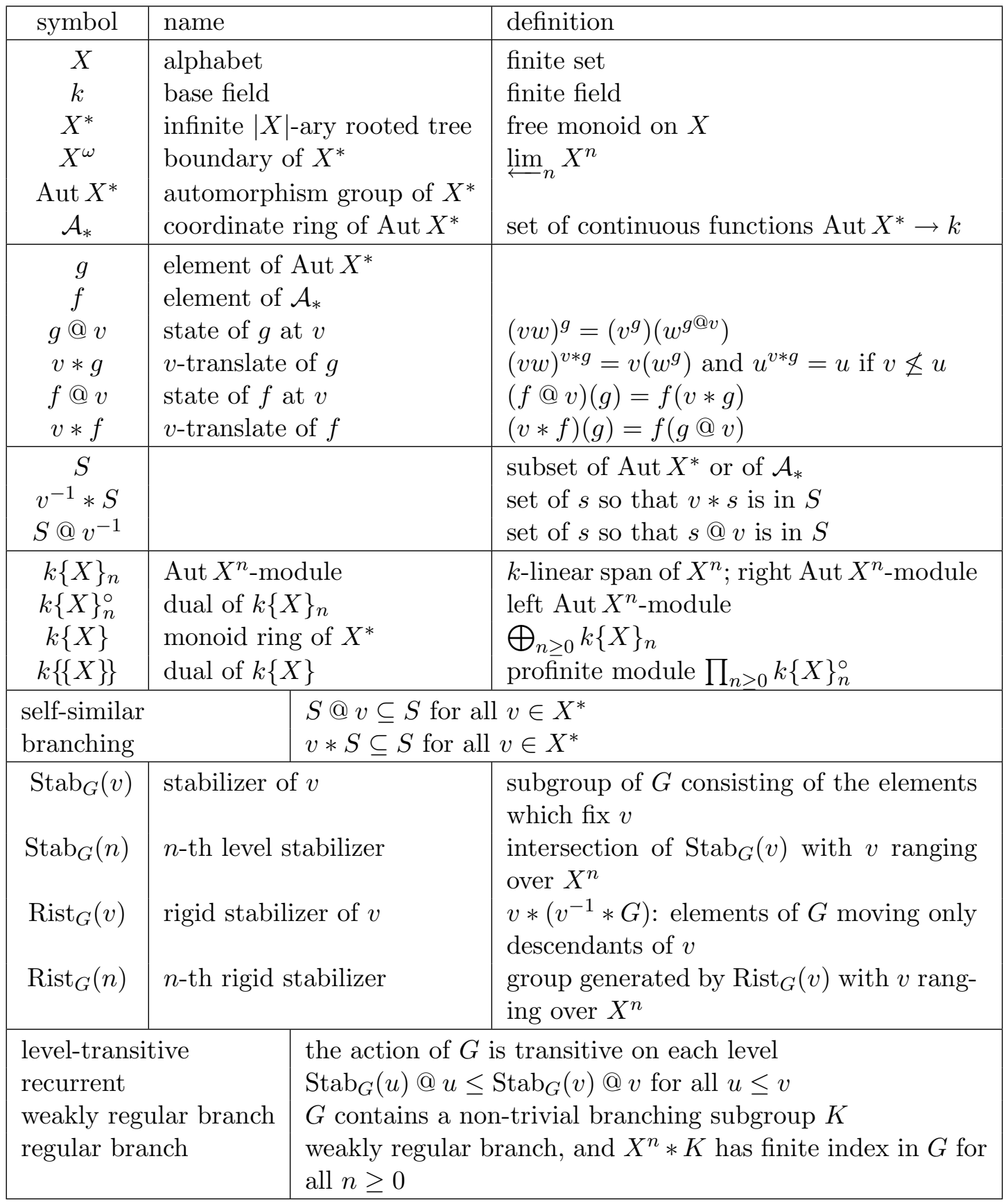

Table 1.1: Symbols, subgroups, and main properties of groups acting on regular rooted trees. 



\section{Chapter 2}

\section{The Special Case $\operatorname{Aut}_{p} X^{*}$}

We suppose $X$ has $p$ letters and consider the group $\operatorname{Aut}_{p} X^{*}=\operatorname{Aut}_{A} X^{*}$ where $A$ is a cyclic group of order $p$ acting on $X$. This group is of crucial importance for at least two reasons: many concrete examples of groups acting on rooted trees are actually subgroups of $\operatorname{Aut}_{p} X^{*}$ and, as a distinctive feature, $\operatorname{Aut}_{p} X^{*}$ is a pro- $p$ group. This allows us to refine some results of Chapter 1; see for example Theorem 2.2.9. Also, we are able to state quite general conditions to ensure a self-similar group is torsion, or torsion-free; see Sections 2.6 and 2.8. In case $p=2$, there is no distinction between $\operatorname{Aut}_{p} X^{*}$ and Aut $X^{*}$.

In Section 2.1 we explain the formalism suited to the study of $\operatorname{Aut}_{p} X^{*}$. After what we established in Chapter 1, not much more is needed. In Section 2.2 we adapt the results of Chapter 1 to our new setting. This is just a very little formal change in most cases. Nevertheless, this yields a quick overview of the results we obtained so far, illustrated with examples. Moreover, we can improve Theorem 1.8.6 for $\operatorname{Aut}_{p} X^{*}$. In Section 2.3 we introduce the crucial notion of nice generating sets. We further develop this point in Section 2.4. Section 2.5 paves the way for the last three sections: Sections 2.6 and 2.8 contain some conditions for a self-similar group to be torsion-free or torsion. For the latter we need to introduce bounded and spinal automorphisms; this is done in Section 2.7.

\subsection{Definition}

We suppose the cardinality of $X$ is a prime number $p$. We let $k$ be the field $\mathbb{F}_{p}$ with $p$ elements and we fix a bijection $\mathbb{F}_{p} \leftrightarrow X$. For an element $x \in \mathbb{F}_{p}$, we denote by $\underline{x}$ its image in $X$. Addition of 1 in $\mathbb{F}_{p}$ induces, via this bijection, a permutation of $X$ which we write $\sigma$. Let $A \leq \operatorname{Sym}(X)$ be the cyclic group of order $p$ generated by $\sigma$. In Section 1.9 we defined $\operatorname{Aut}_{A} X^{*}$ as the group consisting of the elements $g$ satisfying $\pi_{1}(g @ v) \in A$ for all $v \in X^{*}$. For this particular choice of $A$, we shall write $\mathrm{Aut}_{p} X^{*}=\operatorname{Aut}_{A} X^{*}$. Up to isomorphism, this group does not depend on the bijection $\mathbb{F}_{p} \leftrightarrow X$.

The group $\operatorname{Aut}_{p} X^{*}$ is the inverse limit of the groups $\operatorname{Aut}_{p} X^{n}$, which are $p$ Sylow subgroups of Aut $X^{n}$. Thus $\operatorname{Aut}_{p} X^{*}$ is a pro- $p$-Sylow subgroup of Aut $X^{*}$; in particular it is a pro- $p$ group. A basis of neighbourhoods of the identity is 
$\left\{\operatorname{Stab}_{\mathrm{Aut}_{p} X^{*}}(n) \mid n \geq 0\right\}$.

Let $\mathcal{P}_{n}$ be the algebra of functions $\operatorname{Aut}_{p} X^{n} \rightarrow \mathbb{F}_{p}$ and let $\mathcal{P}_{*}$ be the algebra of continuous functions $\operatorname{Aut}_{p} X^{*} \rightarrow \mathbb{F}_{p}$. We shall write $\mathcal{I}_{p}(S)$ for the annihilator of $S$ in $\mathcal{P}_{*}$, and $\mathcal{V}_{p}(I)$ for the annihilator of $I$ in $\operatorname{Aut}_{p} X^{*}$. It is easy to see that $\operatorname{Aut}_{p} X^{*}$ is both self-similar and branching (see Section 1.9). Therefore the operations @ and $*$ descend to $\operatorname{Aut}_{p} X^{*}$, and we define the dual operations on $\mathcal{P}_{*}$ as we did in Section 1.3. Self-similarity and branching are defined as in Section 1.4.

We define the activity function

$$
\text { Act } \begin{aligned}
: A & \rightarrow \mathbb{F}_{p} \\
\sigma^{n} & \mapsto \operatorname{Act}\left(\sigma^{n}\right)=n .
\end{aligned}
$$

It is clear that the algebra of functions $A \rightarrow \mathbb{F}_{p}$ is generated by Act. This follows from Lagrange interpolation. More precisely, every function $A \rightarrow \mathbb{F}_{p}$ can be represented in a unique way as a polynomial in Act, of degree at most $p-1$.

We define the linear map

$$
\begin{aligned}
{[\bullet]: \mathbb{F}_{p}\{X\} } & \rightarrow \mathcal{P}_{*} \\
m & \mapsto[m]=m * \text { Act } .
\end{aligned}
$$

A special case is $[v]=v *$ Act with $v \in X^{*}$. We say $[v](g)$ is the activity of $g$ at the vertex $v$. In case $[v](g) \neq 0$, we say $g$ is active at $v$. The action of $\mathbb{F}_{p}\{X\}$ on $\operatorname{Aut}_{p} X^{*}$ yields $v *[w]=[v w]$. Note that with our conventions, the depth of $[v]$ is $|v|+1$.

Lemma 2.1.1. $\mathcal{P}_{*}$ is generated by $[\varnothing]$ as a branching algebra.

Proof. $[\varnothing]$ generates $\mathcal{P}_{1}$, and therefore we can prove the analogue of Lemma 1.5.4, which implies the claim.

Corollary 2.1.2. Every continuous function Aut $_{p} X^{*} \rightarrow \mathbb{F}_{p}$ can be represented as a polynomial in the variables $[v]$, of degree at most $p-1$ in each variable.

\subsection{Subgroups of $\operatorname{Aut}_{p} X^{*}$}

The results of Section 1.7 remain valid when we replace Aut $X^{*}$ by $\operatorname{Aut}_{p} X^{*}, \mathcal{A}_{*}$ by $\mathcal{P}_{*}, \mathcal{I}$ by $\mathcal{I}_{p}$, and $\mathcal{V}$ by $\mathcal{V}_{p}$. We state the " $p$-versions" of these results below. We omit the proofs, since they are identical to the original ones.

We define an action

$$
\begin{aligned}
\mathcal{P}_{*} \times \operatorname{Aut}_{p} X^{*} & \rightarrow \mathcal{P}_{*} \\
(f, g) & \mapsto f^{g}
\end{aligned}
$$

of $\operatorname{Aut}_{p} X^{*}$ on $\mathcal{P}_{*}$ by algebra endomorphisms, via the formula

$$
f^{g}(h)=f(g h) .
$$

As for Aut $X^{*}$, this action preserves depth. 
Example 2.2.1. It is easy to compute the $\operatorname{Aut}_{p} X^{*}$-action on $[v]$, because $[\varnothing]$ is a group homomorphism on $\operatorname{Aut}_{p} X^{*}$. Therefore we have

$$
[v]^{g}=[v](g)+\left[v^{g}\right] .
$$

Indeed, this is just another way to write the cocycle relation $[v](g h)=[v](g)+$ $\left[v^{g}\right](h)$, see Lemma 1.3.1.

Proposition 2.2.2. Let $G$ be a closed subset of $\operatorname{Aut}_{p} X^{*}$ and write $I=\mathcal{I}_{p}(G)$. Then $G$ is a group if and only if $I^{g} \subseteq I$ for all $g \in G$.

Proposition 2.2.3. Let $G \leq \operatorname{Aut}_{p} X^{*}$ be a group generated by a set $S$ and consider a set $I \subseteq \mathcal{P}_{*}$ so that $f(1)=0$ and $f^{s}-f \in \mathcal{I}_{p}(G)$ for all $f \in I$ and $s \in S$. Then $I \subseteq \mathcal{I}_{p}(G)$.

Proposition 2.2.4. Let $G$ be a closed subgroup of $\mathrm{Aut}_{p} X^{*}$ and consider a function $f \in \mathcal{P}_{*}$. Then $f: G \rightarrow \mathbb{F}_{p}$ is a group homomorphism if and only if $f^{g} \equiv f+f(g)\left(\bmod \mathcal{I}_{p}(G)\right)$ for all $g \in G$.

Lemma 2.2.5. Let $G \leq H$ be closed subgroups of $\operatorname{Aut}_{p} X^{*}$. The following are equivalent

- $G$ has finite index in $H$;

- There is a finite set $S \subseteq \mathcal{P}_{*}$ so that $G=\mathcal{V}_{p}\left(\mathcal{I}_{p}(H)+(S)\right)$.

Example 2.2.6. We consider $p=2$. The Grigorchuk group $\mathrm{G}$ is generated by $S=\{a, b, c, d\}$, see Section A.1. Since G is self-similar, so is its closure, and $\mathcal{I}(\mathrm{G})$ is branching by Proposition 1.4.2.

Write $\Delta^{n}=\sum_{v \in X^{n}} v$ and define

$$
\begin{aligned}
P_{\Delta} & =\left[\Delta^{1}+\Delta^{2}+\Delta^{3}\right], \\
P_{0} & =\left[\underline{0}+\underline{1} *\left(\Delta^{1}+\Delta^{2}\right)\right], \\
Q & =[\underline{0}] \cdot[\underline{1}]+\left[\Delta^{1} * \underline{1}+\Delta^{1} * \underline{0} * \Delta^{1}\right] .
\end{aligned}
$$

We claim $\mathcal{I}(\mathrm{G})$ contains $P_{\Delta}, P_{0}$ and $Q$. To prove this, consider the ideals $I_{1}=$ $\left(P_{\Delta}\right), I_{2}=\left(P_{\Delta}, P_{0}\right)$ and $I_{3}=\left(P_{\Delta}, P_{0}, Q\right)$. We use Equation (2.2.1) to compute the action of $S$ on $I_{d}$. Thus we check

$$
\begin{gathered}
P_{\Delta}^{s}-P_{\Delta}=0, \\
P_{0}^{s}-P_{0} \in I_{1}, \\
Q^{s}-Q \in I_{2},
\end{gathered}
$$

for all $s \in S$. The first equation implies $I_{1} \subseteq \mathcal{I}(\mathrm{G})$, the second implies $I_{2} \subseteq \mathcal{I}(\mathrm{G})$, and the third one implies $I_{3} \subseteq \mathcal{I}(\mathrm{G})$. More details are given in the proof of Proposition A.1.1, which states that $I_{3}$ even generates $\mathcal{I}(\mathrm{G})$ as a branching ideal.

Theorem 1.8 .2 also holds in the $p$-setting, with identical proof:

Theorem 2.2.7. Let $G$ be a closed, self-similar subgroup of Aut $_{p} X^{*}$. Then $G$ is regular branch if and only if $\mathcal{I}_{p}(G)$ is finitely generated as a branching ideal. 


\subsection{Nice Generating Sets}

Corollary 2.2.8. Let $G \leq \operatorname{Aut}_{p} X^{*}$ be a (not necessarily closed) self-similar group, regular branch over $K$, and suppose $K$ contains $\operatorname{Stab}_{G}(d-1)$. Then $\mathcal{I}_{p}(G)$ is generated by $\mathcal{I}_{p}(G) \cap \mathcal{P}_{d}$ as a branching ideal.

On the other hand, the analogue of Theorem 1.8.6 is stronger for subgroups of $\operatorname{Aut}_{p} X^{*}$, because we have more control on generating sets.

Let $G$ be a closed subgroup of $\operatorname{Aut}_{p} X^{*}$. As $G$ is pro- $p$, we know that $G$ is finitely generated if and only if its Frattini quotient $G / \Phi(G)$ is finitely generated, see [RZ00, Proposition 2.8.10]. Another way to say this: there are only finitely many independent continuous homomorphisms from $G$ to $\mathbb{F}_{p}$.

Theorem 2.2.9. Let $G$ be a closed subgroup of $\operatorname{Aut}_{p} X^{*}$ which is self-similar and regular branch, and let $d$ be the depth of $\mathcal{I}_{p}(G)$. Then $G$ is topologically finitely generated if and only if there is no group homomorphism $G \rightarrow \mathbb{F}_{p}$ of $G$-depth at least $d$.

Proof. If there is such a homomorphism, the same proof as for Theorem 1.8.6 shows that $G$ is not finitely generated. If there is no such homomorphism, then there are finitely many continuous homomorphisms $G \rightarrow \mathbb{F}_{p}$, and so $G$ is finitely generated.

Example 2.2.10. We continue Example 2.2 .6 concerning the Grigorchuk group $\mathrm{G}$. Write $\overline{\mathrm{G}_{1}}=\mathcal{V}\left(X^{*} * I_{1}\right)$ and $\overline{\mathrm{G}_{2}}=\mathcal{V}\left(X^{*} * I_{2}\right)$. These are easily seen to be self-similar groups, whose ideals both have depth 4 . It is clear that $P_{0}$ (resp. $Q$ ) is a group epimorphism $\overline{\mathrm{G}_{1}} \rightarrow \mathbb{F}_{2}$ (resp. $\overline{\mathrm{G}_{2}} \rightarrow \mathbb{F}_{2}$ ) of depth 4 . Therefore these two groups are not finitely generated (in the topological sense), due to the theorem. On the other hand, $\overline{\mathrm{G}}_{3}=\mathcal{V}\left(X^{*} * I_{3}\right)$ contains $\mathrm{G}$ as a dense subgroup, see Proposition A.1.1.

\subsection{Nice Generating Sets}

The ideal associated to a subgroup of $\operatorname{Aut}_{p} X^{*}$ always admits nice generating sets; some examples can be found in Appendix A. We give the definition and then prove that these nice generating sets always exist.

Consider the vector space $\left[\mathbb{F}_{p}\{X\}_{n}\right]$ spanned by $\left\{[v] \mid v \in X^{n}\right\}$. An element $f \in \mathcal{P}_{*}$ of depth $n$ is nice if there is $f_{1} \in \mathcal{P}_{n-1}$ and $f_{2} \in\left[\mathbb{F}_{p}\{X\}_{n-1}\right]$ so that $f=f_{1}+f_{2}$. The function $f_{2}$ is called the linear part of $f$, and shall be written $\operatorname{lin}(f)$.

Proposition 2.3.1. Let $G$ be a closed subgroup of $\mathrm{Aut}_{p} X^{*}$ and let $f: G \rightarrow \mathbb{F}_{p}$ be a continuous group homomorphism. Then $f$ can be realised as a nice function.

Proof. If $f$ is the trivial homomorphism then there is nothing to prove, so we suppose $f$ is non-trivial. Thus $f$ is surjective and its kernel $N$ is a maximal subgroup of $G$; moreover $N$ is closed in $\operatorname{Aut}_{p} X^{*}$.

Let $n$ be the maximal integer so that the index of $\operatorname{Stab}_{N}(n)$ in $\operatorname{Stab}_{G}(n)$ is equal to $p$. In other words we choose $n$ maximal so that $\pi_{n}(G)=\pi_{n}(N)$. We let $T$ be a transversal of $\operatorname{Stab}_{N}(n)$ in $N$, and $R$ a transversal of $\operatorname{Stab}_{N}(n)$ in $\operatorname{Stab}_{G}(n)$. 
Note that by the choice of $n$, the set $T$ is also a transversal of $\operatorname{Stab}_{G}(n)$ in $G$. Then it is clear that any element of $G$ can be written in a unique way as srt with $s \in \operatorname{Stab}_{N}(n), r \in R$ and $t \in T$.

The map $f$ induces a group homomorphism $\operatorname{Stab}_{G}(n) \rightarrow \mathbb{F}_{p}$ with kernel $\operatorname{Stab}_{N}(n)$; this is due to the choice of $n$. Viewing $\pi_{n+1}\left(\operatorname{Stab}_{G}(n)\right)$ as a vector space over $\mathbb{F}_{p}$, the map $f$ induces a linear functional $f_{2}: \pi_{n+1}\left(\operatorname{Stab}_{G}(n)\right) \rightarrow \mathbb{F}_{p}$. This defines $f_{2} \in\left[\mathbb{F}_{p}\{X\}\right]$. We now choose a function $f_{1} \in \mathcal{P}_{n}$ so that $f_{1}(t)=-f_{2}(t)$ for all $t \in T$, and write $f^{\prime}=f_{1}+f_{2}$. We claim $f$ coincides with $f^{\prime}$ on $G$.

Using the decomposition $g=s r t$ above, it is clear that we have $f(s r t)=f(r)$, because $s$ and $t$ are in $N=\operatorname{ker} f$. Moreover, we have $f(r)=f_{2}(r)$. On the other hand, Equation (2.2.1) yields

$$
f_{2}(s r t)=f_{2}^{s}(r t)=f_{2}(s)+f_{2}(r t)=f_{2}^{r}(t)=f_{2}(r)+f_{2}(t) .
$$

Since $f_{1}$ is in $\mathcal{P}_{n}$, it only depends on $t$ : $f_{1}(s r t)=f_{1}(t)$. Therefore we have $f^{\prime}(s r t)=f_{1}(s r t)+f_{2}(s r t)=f_{1}(t)+f_{2}(r)+f_{2}(t)=f_{2}(r)$, and thus $f(g)=f^{\prime}(g)$ for all $g \in G$.

We use this proposition to prove the existence of nice generating sets. A nice generating set for the ideal $\mathcal{I}_{p}(G)$ of a group $G \leq \operatorname{Aut}_{p} X^{*}$ is a collection $S \subseteq \mathcal{P}_{*}$ satisfying the following properties:

(i) $\mathcal{V}_{p}(S)$ is the closure of $G$ and $S$ consists of nice functions;

(ii) $S$ is well-ordered, and the order is compatible with depth: $S=\left\{f_{n} \mid n=\right.$ $1,2, \ldots\}$ and $i \leq j \operatorname{implies} \operatorname{depth}\left(f_{i}\right) \leq \operatorname{depth}\left(f_{j}\right)$;

(iii) for each $n \geq 0$, the set $G_{n}=\mathcal{V}_{p}\left(\left\{f_{i}: 1 \leq i \leq n\right\}\right)$ is a group;

(iv) for all $0 \leq i \leq j$, the index of $G_{j}$ in $G_{i}$ is equal to $p^{j-i}$.

Definition 2.3.2. The function $f_{1}$ is called the principal function of $\mathcal{I}_{p}(G)$, or (abusively) of $G$.

The principal function of a group is unique up to scalar multiple, as we shall see in the proof of the next proposition.

Proposition 2.3.3. Let $G$ be a subgroup of $\operatorname{Aut}_{p} X^{*}$. Then $\mathcal{I}_{p}(G)$ has a nice generating set.

Proof. We set $G_{n, 0}=\pi_{n}^{-1}\left(\pi_{n}(G)\right)$ for all $n \geq 0$, and from this we shall build the rest of the nice generating set. For each $n \geq 0$, choose a refined chain of subgroups between $G_{n, 0}$ and $G_{n+1,0}$, and label it as

$$
G_{n, 0}>G_{n, 1}>\cdots>G_{n, d_{n}}=G_{n+1,0} .
$$

Since $G_{n, 0}$ and $G_{n+1,0}$ contain $\operatorname{Stab}(n+1)$, this can be viewed modulo $\operatorname{Stab}(n+1)$, dealing only with finite $p$-groups. It is a standard fact that a maximal subgroup of a $p$-group has index $p$ and is the kernel of an epimorphism onto $\mathbb{F}_{p}$. Therefore the index of $G_{n, i+1}$ in $G_{n, i}$ is equal to $p$ and there is a continuous group epimorphism 


\subsection{Uniseriality}

$f_{n, i+1}: G_{n, i} \rightarrow \mathbb{F}_{p}$ with kernel $G_{n, i+1}$. By Proposition 2.3.1, $f_{n, i+1}$ can be written as a nice function. We order the $f_{n, i}$ using lexicographic order on the double index. Relabelling the $f_{n, i}$ with natural numbers, it is straightforward to check that $S=\left\{f_{n, i}\right\}$ is a nice generating set for $\mathcal{I}_{p}(G)$.

\subsection{Uniseriality}

We define a function

$$
\Delta_{\bullet}:\{0, \ldots, p-1\}^{*} \rightarrow \mathbb{F}_{p}\{X\}
$$

which sends the $p$-adic expansion $w=x_{1} \ldots x_{n}$ to the element

$$
\Delta_{w}=\sum_{i_{1}, \ldots, i_{n}=0}^{p-1}\left(\begin{array}{c}
i_{1} \\
p-1-x_{1}
\end{array}\right) \cdots\left(\begin{array}{c}
i_{n} \\
p-1-x_{n}
\end{array}\right) \underline{i_{1}} \cdots \underline{i_{n}} .
$$

Clearly, $\Delta_{\bullet}$ is a monoid homomorphism:

$$
\Delta_{v} * \Delta_{w}=\Delta_{v w}
$$

We reserve the symbol $\Delta$ without subscript for the special value $\Delta=\Delta_{p-1}=$ $\sum_{x \in X} x$. We then have

$$
\Delta^{n}=\Delta * \cdots * \Delta=\sum_{v \in X^{n}} v
$$

and we set $\Delta^{0}=\varnothing$. This agrees with the notation introduced in Section 1.8. We also point out the relation $\Delta_{0^{n}}=(\underline{p-1})^{n}$.

Proposition 2.4.1. $\mathbb{F}_{p}\{X\}$ is freely generated by $\left\{\Delta_{0}, \ldots, \Delta_{p-1}\right\}$.

Proof. The algebra $\mathbb{F}_{p}\{X\}$ is freely generated by $X$. Because it is graded, any free basis of $\mathbb{F}_{p}\{X\}$ must consist of $p$ elements of depth 1. Better, any $p$ linearly independent elements of degree 1 form a basis of $\mathbb{F}_{p}\{X\}$.

A module is uniserial if it has only one composition series. Equivalently, a module is uniserial if the collection of its submodules is totally ordered. In [Ple83], Plesken already noted that $\mathbb{F}_{p}\{X\}_{n}$ is a uniserial $\operatorname{Aut}_{p} X^{n}$-module. We explicit its submodules, and state further results from Plesken's article.

We define the degree:

$$
\begin{aligned}
\operatorname{deg}:\{0, \ldots, p-1\}^{*} & \rightarrow \mathbb{N} \\
x_{1} \ldots x_{n} & \mapsto \sum_{i=1}^{n}\left(p-1-x_{i}\right) p^{i-1} .
\end{aligned}
$$

It is readily checked that this function satisfies

$$
\operatorname{deg}(v w)=\operatorname{deg}(v)+p^{|v|} \operatorname{deg}(w) .
$$


Let $M_{d}^{(n)}$ be the $\mathbb{F}_{p^{-}}$span of $\left\{\Delta_{w} \mid w\right.$ has length $n$ and $\left.\operatorname{deg}(w)<d\right\}$. One can check that $M_{d}^{(n)}$ has dimension $d$, and that it is an Aut $_{p} X^{n}$-submodule of $\mathbb{F}_{p}\{X\}_{n}$. Thus $\mathbb{F}_{p}\{X\}_{n}$ admits the composition series

$$
0=M_{0}^{(n)}<M_{1}^{(n)}<\cdots<M_{p^{n}-1}^{(n)}<M_{p^{n}}^{(n)}=\mathbb{F}_{p}\{X\}_{n}
$$

where the quotient of two consecutive modules has dimension one. Moreover, this is the unique composition series of $\mathbb{F}_{p}\{X\}_{n}$.

The property that $\mathbb{F}_{p}\{X\}_{n}$ is uniserial is not shared by all subgroups of $\operatorname{Aut}_{p} X^{n}$. Plesken found a simple characterization of the groups which possess this property. Translated in our language, his result is

Proposition 2.4.2 ([Ple83, Corollary 2]). Let $G \leq \operatorname{Aut}_{p} X^{*}$ be a group. Then $\mathbb{F}_{p}\{X\}_{n}$ is a uniserial $G$-module if and only if $\left[\Delta^{m}\right](G)=\mathbb{F}_{p}$ for all $0 \leq m<n$.

In case $\mathbb{F}_{p}\{X\}_{n}$ is uniserial, the only $G$-submodules of $\mathbb{F}_{p}\{X\}_{n}$ are given in Equation (2.4.1). We may even refine Proposition 2.4.2 using the following:

Proposition 2.4.3 ([Ple83, Proposition 5]). For $0<d<p^{n}$, the kernel of the action of $\operatorname{Aut}_{p} X^{n}$ on $M_{d+1}^{(n)} / M_{d-1}^{(n)}$ is $\operatorname{ker}\left[\Delta^{\mu(d)}\right]$, where $p^{\mu(d)}$ is the biggest power of $p$ which divides $d$.

Corollary 2.4.4. Let $G \leq \operatorname{Aut}_{p} X^{*}$ be a group. Then $M_{d}^{(n)}$ is a uniserial $G$ module if and only if $\left[\Delta^{l}\right](G)=\mathbb{F}_{p}$ for $0 \leq l \leq\left\lfloor\log _{p}(d-1)\right\rfloor$, where $\lfloor x\rfloor$ is the greatest integer less than or equal to $x$.

A consequence of the uniseriality is a unicity result for nice generating sets.

Proposition 2.4.5. Let $G \leq \operatorname{Aut}_{p} X^{*}$ be a group so that $\left[\Delta^{n}\right](G)=\mathbb{F}_{p}$ for all $n \geq 0$, and let $\left\{f_{i} \mid i>0\right\}$ and $\left\{f_{i}^{\prime} \mid i>0\right\}$ be nice generating sets for $\mathcal{I}_{p}(G)$. Then the ideals $\left(f_{1}, \ldots, f_{d}\right)$ and $\left(f_{1}^{\prime}, \ldots, f_{d}^{\prime}\right)$ coincide for all $d \geq 0$.

Proof. We use the same notation as in Proposition 2.3.3. The choice of a nice generating set induces the choice of a refined chain of subgroups $G_{n, i}$ between $\operatorname{Aut}_{p} X^{*}$ and the closure of $G$. This choice, in turn, is equivalent to the choice of a refined chain of subgroups between $\pi_{n+1}(\operatorname{Stab}(n))$ and $\pi_{n+1}\left(\operatorname{Stab}_{G}(n)\right)$ for all $n \geq 0$.

The group $\pi_{n+1}(\operatorname{Stab}(n))$ is an elementary Abelian $p$-group, and it is normal in $\pi_{n+1}(G)$. We identify it with the module $\mathbb{F}_{p}\{X\}_{n}$. Since the latter is uniserial, there is only one possible choice for the chain of subgroups. This implies that the groups $G_{n, i}$ are uniquely determined, and so are the corresponding ideals $\mathcal{I}_{p}\left(G_{n, i}\right)$. These are exactly the ideals from the claim, after renumbering.

Example 2.4.6. We continue Examples 2.2.6 and 2.2.10, which concern the Grigorchuk group G. By Proposition 2.4.5, the ideals $I_{1}, I_{2}$ and $I_{3}$ are in fact independent of the nice generating set, and thus canonically associated to $\mathrm{G}$. Therefore the self-similar groups $\overline{\mathrm{G}_{1}}=\mathcal{V}\left(X^{*} * I_{1}\right)$ and $\overline{\mathrm{G}_{2}}=\mathcal{V}\left(X^{*} * I_{2}\right)$ are also canonically associated to $\mathrm{G}$. We will study the group $\overline{\mathrm{G}_{2}}$ more closely in Chapter 7 . 


\subsection{Signature and Maximal Subgroups}

\subsection{Signature and Maximal Subgroups}

To any subgroup of $\operatorname{Aut}_{p} X^{*}$ one can associate a vector space in the following way. First, observe that the map

$$
\begin{aligned}
\Sigma: \operatorname{Aut}_{p} X^{*} & \rightarrow \mathbb{F}_{p}^{\mathbb{N}} \\
g & \mapsto\left(\left[\Delta^{0}\right](g),\left[\Delta^{1}\right](g), \ldots\right)
\end{aligned}
$$

is a group homomorphism, whose kernel is the Frattini subgroup of $\operatorname{Aut}_{p} X^{*}$. By restriction, this map associates to any group $G$ its signature $\Sigma(G)$. This subspace of $\mathbb{F}_{p}^{\mathbb{N}}$ does not change if $G$ is replaced by a conjugate in $\operatorname{Aut}_{p} X^{*}$. The co-signature $\Sigma^{\perp}(G)$ of $G$ is the set of continuous linear functionals vanishing on $\Sigma(G)$. Thus we see $\Sigma^{\perp}(G)$ as a subspace of $\mathbb{F}_{p}^{\mathbb{N}}$, or even better, as a subspace of $\left[\mathbb{F}_{p}\{\Delta\}\right]$ : the linear span of $\left\{\left[\Delta^{0}\right],\left[\Delta^{1}\right], \ldots\right\}$.

Lemma 2.5.1. Let $G$ be a closed subgroup of $\operatorname{Aut}_{p} X^{*}$. We have the equality $\Sigma^{\perp}(G)=\mathcal{I}_{p}(G) \cap\left[\mathbb{F}_{p}\{\Delta\}\right]$.

Proof. Follows directly from the definitions.

We define the left shift:

$$
\begin{aligned}
\mathbb{F}_{p}^{\mathbb{N}} & \rightarrow \mathbb{F}_{p}^{\mathbb{N}} \\
\left(x_{0}, x_{1}, x_{2}, \ldots\right) & \mapsto\left(x_{1}, x_{2}, \ldots\right)
\end{aligned}
$$

and the right shift:

$$
\begin{aligned}
\mathbb{F}_{p}^{\mathbb{N}} & \rightarrow \mathbb{F}_{p}^{\mathbb{N}} \\
\left(x_{0}, x_{1}, \ldots\right) & \mapsto\left(0, x_{0}, x_{1}, \ldots\right) .
\end{aligned}
$$

Proposition 2.5.2. If $G$ is a closed self-similar subgroup of $\operatorname{Aut}_{p} X^{*}$, then $\Sigma(G)$ is invariant under left shift, and $\Sigma^{\perp}(G)$ is invariant under right shift.

Proof. For any element $g \in G$, the element $\prod_{x \in X} g @ x$ is in $G$ (with the product in any order). We compute

$$
\left[\Delta^{n}\right]\left(\prod_{x \in X} g @ x\right)=\sum_{x \in X}\left[\Delta^{n}\right](g @ x)=\left[\Delta * \Delta^{n}\right](g)=\left[\Delta^{n+1}\right](g) .
$$

Therefore $\Sigma(G)$ is invariant under left shift.

By Proposition 1.4.2 we know that $\mathcal{I}_{p}(G)$ is branching. Also, Lemma 2.5.1 says $\Sigma^{\perp}(G)=\mathcal{I}_{p}(G) \cap\left[\mathbb{F}_{p}\{\Delta\}\right]$. Therefore if $\sum_{n \geq 0} a_{n}\left[\Delta^{n}\right]$ is in $\mathcal{I}_{p}(G)$, then $\Delta * \sum_{n>0} a_{n}\left[\Delta^{n}\right]=\sum_{n>0} a_{n}\left[\Delta^{n+1}\right]$ is also in $\mathcal{I}_{p}(G)$, and in $\left[\mathbb{F}_{p}\{\Delta\}\right]$, too. Thus $\Sigma^{\perp}(G)$ is invariant under right shift.

Proposition 2.5.3. If $G$ is a closed branching subgroup of $\operatorname{Aut}_{p} X^{*}$, then $\Sigma(G)$ is invariant under right shift, and $\Sigma^{\perp}(G)$ is invariant under left shift.

Proof. Similar as Proposition 2.5.2. 
The closed normal subgroups of Aut $X^{*}$ have been investigated in [BORT96]. Within our setup, the maximal closed subgroups of $\mathrm{Aut}_{p} X^{*}$ are easily described.

Proposition 2.5.4. A closed subgroup $G$ of $\operatorname{Aut}_{p} X^{*}$ is maximal if and only if $\Sigma^{\perp}(G)$ is one-dimensional and $\mathcal{I}_{p}(G)$ is generated by $\Sigma^{\perp}(G)$.

Proof. Since $\operatorname{Aut}_{p} X^{*}$ is pro- $p$, any maximal closed subgroup has index $p$, and it must contain the Frattini subgroup of $\operatorname{Aut}_{p} X^{*}$. Therefore one can see any maximal subgroup directly in $\Sigma\left(\operatorname{Aut}_{p} X^{*}\right)$. The claim follows.

Corollary 2.5.5. Let $G$ be a subgroup of $\operatorname{Aut}_{p} X^{*}$. Then the principal function of $G$ can be written as $f_{1}=\sum_{n \geq 0} a_{n}\left[\Delta^{n}\right]$ for some coefficients $a_{n} \in \mathbb{F}_{p}$.

Proof. By definition of nice generating sets, if $f_{1}$ is a principal function of $G$, then $G_{1}=\mathcal{V}_{p}\left(f_{1}\right)$ has index $p$ in $\operatorname{Aut}_{p} X^{*}$, so it is maximal.

\subsection{Torsion-Free Groups}

Let $G$ be a closed subgroup of $\operatorname{Aut}_{p} X^{*}$. Recall (Definition 2.3.2 and Corollary 2.5.5) that the principal function of $G$ is the non-zero function $\sum_{n \geq 0} a_{n}\left[\Delta^{n}\right]$ in $\mathcal{I}_{p}(G)$ which has minimal depth. This function is unique, up to scalar multiple.

Proposition 2.6.1. Let $G \leq \operatorname{Aut}_{p} X^{*}$ be a self-similar group and let $f=$ $\sum_{n \geq 0} a_{n}\left[\Delta^{n}\right]$ be its principal function. If $a_{0} \neq 0$ then $G$ is torsion-free.

Proof. Suppose $a_{0} \neq 0$. If $a_{n}=0$ for all $n>0$ then $G$ is trivial and thus torsionfree. Otherwise let $g \in G$ be non-trivial. We shall prove $g^{p}$ is non-trivial. Since Aut $_{p} X^{*}$ is residually- $p$, this will prove that any non-trivial $g$ has infinite order.

Replacing $g$ by $g @ v$ for some $v$, we may suppose $[\varnothing](g) \neq 0$. Because $f(g)=0$, we must have $\left[\Delta^{n}\right](g) \neq 0$ for some $n>0$. Using Equation (2.2.1), we compute

$$
[x v]\left(g^{p}\right)=[x v](g)+\left[(x v)^{g}\right](g)+\cdots+\left[(x v)^{g^{p-1}}\right](g) .
$$

Therefore the relation $\left[x * \Delta^{n-1}\right]\left(g^{p}\right)=\left[\Delta^{n}\right](g)$ holds for all $x \in X$. Thus $\left[x * \Delta^{n-1}\right]\left(g^{p}\right)$ is non-zero, and so $g^{p}$ is non-trivial.

Example 2.6.2. Let $A$ be the group generated by

$$
\tau=(1, \tau) \sigma
$$

See Section A.6. The element $\tau$ is called the "adding machine" because its action on $X^{n}$ just adds 1 in base 2. It is straightforward to check that the principal function of $A$ is $[\varnothing+\Delta]$. Therefore $A$ is torsion-free. Since $A$ is cyclic, it is isomorphic to $\mathbb{Z}$.

More generally, we have the following

Corollary 2.6.3. Any self-similar group generated by conjugates of the adding machine is torsion-free. 


\subsection{Some Specific Classes of Automorphisms}

Proof. The function $[\varnothing-\Delta]$ is a group homomorphism Aut $_{p} X^{*} \rightarrow \mathbb{F}_{p}$, and the adding machine $\tau=(1, \ldots, 1, \tau) \sigma$ lies in its kernel. Therefore so does any conjugate of the adding machine. If the group is self-similar, we can apply the proposition.

Example 2.6.4. The group $G_{\mathrm{BSV}}$ is generated by

$$
\tau=(1, \tau) \sigma \quad \text { and } \quad \mu=\left(1, \mu^{-1}\right) \sigma,
$$

see Section A.7. The automorphism $\mu$ is a conjugate of $\tau$, and the group $G_{\mathrm{BSV}}$ is self-similar. Therefore $G_{\mathrm{BSV}}$ is torsion-free.

The following example shows that the hypothesis of Proposition 2.6.1 is not a necessary condition.

Example 2.6.5. Consider the elements defined by the following recursion

$$
a=(b, b) \sigma, \quad b=(a, 1) \sigma,
$$

and let $G$ be the group generated by $a$ and $b$. Clearly $G$ is self-similar, and one can check that it satisfies $\left[\Delta^{2}\right]$ and $[\varnothing+\Delta+\underline{0} * \Delta]$, the former being the principal function of $G$. By a similar reasoning as in the proof of the proposition, the group $G$ is torsion-free, although it does not satisfy the hypothesis $a_{0} \neq 0$.

\subsection{Some Specific Classes of Automorphisms}

Following Sidki [Sid00], we define the finitary and bounded automorphisms of $X^{*}$. We also introduce a slight variation of the latter: the spinal automorphisms. We shall see in Section 2.8 that the bounded and spinal subgroups of a closed, self-similar $G \leq \operatorname{Aut}_{p} X^{*}$ often coincide, and that they can have a behaviour which is radically different from that of the whole $G$.

Definition 2.7.1. An element $g \in$ Aut $X^{*}$ is finitary if there is $n \in \mathbb{N}$ such that $g @ v=1$ for all $v \in X^{*}$ of length $\geq n$. The minimal such $n$ is the depth of $g$.

It is easily checked that the product of two finitary elements is finitary, and the inverse of a finitary element is finitary. Therefore the finitary elements form a group which we call the finitary group, written $\mathcal{F}$. The finitary subgroup of a group $G \leq$ Aut $X^{*}$ is $G \cap \mathcal{F}$. The depth of $G \cap \mathcal{F}$ is the depth of the element of greatest depth.

Definition 2.7.2. An element $g \in$ Aut $X^{*}$ is bounded if there is $k \in \mathbb{N}$ such that for all $n$ large enough, $g @ v$ is trivial for all but at most $k$ words $v \in X^{n}$. For the minimal such $k$, we say that $g$ is $k$-bounded.

Definition 2.7.3. An element $g \in$ Aut $X^{*}$ is spinal if there exists a finite set $S \subset X^{\omega}$ such that for every $v \in X^{*} \backslash \operatorname{Prefix}(S)$, the element $g @ v$ is finitary. The minimal such $S$ is the set of spines of $g$. A $k$-spinal element is a spinal element which has $k$ spines. 
Note that the 0-bounded and 0-spinal elements are just the finitary elements. Another characterization of spinality is the following.

Lemma 2.7.4. Let $g \in$ Aut $X^{*}$ be such that there is $k \in \mathbb{N}$ such that for all $n \in \mathbb{N}$, the state $g @ v$ is finitary for all but at most $k$ words $v \in X^{n}$. Then $g$ is $k^{\prime}$-spinal for some $k^{\prime} \leq k$.

Proof. For each $n \in \mathbb{N}$, let $W_{n} \subset X^{n}$ be a set of at most $k$ elements, such that $g @ v$ is finitary for all $v \in X^{n} \backslash W_{n}$. Define $S=\left\{v \in X^{\omega}: \operatorname{Prefix}(v) \cap W_{n} \neq\right.$ $\varnothing$ for all $n \in \mathbb{N}\}$. Then because $\operatorname{Prefix}(S) \cap X^{n} \subseteq W_{n}$ for all $n, S$ has at most $k$ elements.

Consider $v \in X^{*} \backslash \operatorname{Prefix}(S)$. There is $n \in \mathbb{N}$ such that either $\operatorname{Prefix}(v) \cap W_{n}=$ $\varnothing$ or $v \notin \operatorname{Prefix}\left(W_{n}\right)$. In both cases we conclude that $g @ v$ is finitary. Therefore $g$ is $k^{\prime}$-spinal for some $k^{\prime} \leq k$.

Remark 2.7.5. Comparing Lemma 2.7.4 with Definition 2.7.2, we see that every bounded element is spinal.

The following lemma gives a sufficient condition for a spinal element to be bounded.

Lemma 2.7.6. Let $g \in$ Aut $X^{*}$ be a spinal element with set of spines $S \subset X^{\omega}$ and suppose the depth of $g @ v$ is bounded by $d$ when $v$ ranges over $X^{*} \backslash \operatorname{Prefix}(S)$. Then $g$ is bounded.

Proof. The hypothesis implies that $g$ has no activity outside the $d$-neighbourhood of $S$. Thus for every $n \in \mathbb{N}$, the automorphism $g @ v$ is trivial for all but at most $|X|^{2 d} \cdot|S|$ words $v \in X^{n}$.

Lemma 2.7.7. The inverse of a $k$-spinal element is $k$-spinal. The product of a $k_{1}$-spinal element by a $k_{2}$-spinal element is $k$-spinal for some $k \leq k_{1}+k_{2}$. The same holds for $k$-bounded elements.

Proof. We will only prove the lemma for spinal elements. Exactly the same proof holds when one replaces simultaneously "spinal" by "bounded" and "finitary" by "trivial".

Let $g \in$ Aut $X^{*}$ be $k$-spinal. Then for all $n \in \mathbb{N},\left(g^{-1}\right) @ v=\left(g @ g^{-1}(v)\right)^{-1}$ is finitary for all but $k$ words $v \in X^{n}$. Therefore $g^{-1}$ is $k^{\prime}$-spinal for some $k^{\prime} \leq k$. The same argument shows that $g=\left(g^{-1}\right)^{-1}$ is $k^{\prime \prime}$-spinal for some $k^{\prime \prime} \leq k^{\prime}$. Since $k^{\prime \prime}=k$ we have proven that $g^{-1}$ is $k$-spinal.

Consider now $g_{1}, g_{2} \in$ Aut $X^{*}$ which are $k_{1}$ - and $k_{2}$-spinal respectively. Then for all $n \in \mathbb{N},\left(g_{1} g_{2}\right) @ v=\left(g_{1}\right) @ g_{2}(v)\left(g_{2}\right) @ v$ is finitary for all but at most $k_{1}+k_{2}$ words $v \in X^{n}$. Therefore $g_{1} g_{2}$ is $k$-spinal for some $k \leq k_{1}+k_{2}$.

The set of bounded elements forms the bounded group, written $\mathcal{B}$. The bounded subgroup of a group $G \leq$ Aut $X^{*}$ is $G \cap \mathcal{B}$. The set of spinal elements forms the spinal group, written $\mathcal{S}$. The spinal subgroup of a group $G \leq \operatorname{Aut} X^{*}$ is $G \cap \mathcal{S}$.

Proposition 2.7.8. Let $G \leq$ Aut $X^{*}$ be a self-similar group, and suppose the finitary subgroup of $G$ has finite depth. Then the bounded and the spinal subgroup of $G$ coincide. 


\subsection{Torsion Groups}

Proof. Let $n$ be the depth of the finitary subgroup of $G$, and let $G_{b}$ and $G_{s}$ denote the bounded and spinal subgroups of $G$. As mentioned above (Remark 2.7.5), every bounded element is spinal. Therefore $G_{b} \leq G_{s}$ holds. By Corollary 2.8.2 we know that $g @ w$ has depth at most $n$ for all $w \in X^{*} \backslash \operatorname{Prefix}(S)$, where $S$ is the set of spines of $g \in G_{s}$. Lemma 2.7 .6 yields the result.

\subsection{Torsion Groups}

Throughout this section, $\bar{G}$ denotes the closure of $G$ in $\operatorname{Aut}_{p} X^{*}$.

Proposition 2.8.1. Let $G \leq \operatorname{Aut}_{p} X^{*}$ be a self-similar, level-transitive group and suppose there is $v \in X^{n}$ and a function $f=[v]+\sum_{w \in X^{n+1}} w * f_{w}$ in $\mathcal{I}_{p}(G)$, where the $f_{w}$ are in $\mathcal{P}_{*}$. If $g \in \bar{G}$ is active at uw with $w$ of length $n$, then $g$ is active at some vertex $u w^{\prime}$ with $w^{\prime}$ longer than $w$.

Proof. Since $G$ is a group, $f^{g}$ is in $\mathcal{I}_{p}(G)$ for all $g \in G$ by Proposition 2.2.2. Now $f^{g}=\left[v^{g}\right]+[v](g)+\sum_{w \in X^{n+1}}\left(w * f_{w}\right)^{g}$ is again of the form $\left[v^{g}\right]+\sum_{w \in X^{n+1}} w *$ $f_{w}^{\prime}$, and using level-transitivity, we deduce that there is a function $h_{v}=[v]+$ $\sum_{w \in X^{n+1}} w * f_{w}$ in $\mathcal{I}_{p}(G)$ for each $v \in X^{n}$.

Suppose $g$ is active at $u v$ with $v$ of length $n$. Then $\left(u * h_{v}\right)(g)=[u v](g)+$ $\sum_{w \in X^{n+1}}\left(u w * f_{w}\right)(g)=0$ and thus $g$ is active at a descendant of the vertex $u w$ for some $w \in X^{n+1}$.

Corollary 2.8.2. If $G, v$ and $f$ are as in the proposition, then the finitary subgroup of $\bar{G}$ has depth at most $n$.

Proof. Let $g \in \bar{G}$ be a finitary element of depth $d>n$. Choose a vertex $u$ of maximal depth (i.e. $|u|=d-1)$ so that $[u](g) \neq 0$, and write $u=v w$ with $w$ of length $n$. Then $g$ has depth strictly greater than $d$ by the proposition, a contradiction.

Corollary 2.8.3. If $G, v$ and $f$ are as in the proposition, then the bounded and the spinal subgroups of $\bar{G}$ coincide.

Proof. Apply Proposition 2.7.8.

Example 2.8.4. The Grigorchuk group $G$ (see Section A.1) satisfies $P_{0}(\mathrm{G})=0$ with $P_{0}=\left[\underline{0}+\underline{1} *\left(\Delta^{1}+\Delta^{2}\right)\right]$. Therefore the finitary elements in $\overline{\mathrm{G}}$ have depth at most 1 . These are 1 and $a$. Moreover, the bounded and the spinal subgroups of $\bar{G}$ coincide.

Example 2.8.5. The Gupta-Sidki group $\ddot{\Gamma}$ (see Section A.3) satisfies $[-\underline{0}+$ $\left.\Delta_{11}\right](\ddot{\Gamma})=0$. Therefore the finitary elements in $\bar{\Gamma}$ have depth at most 1 . These are $1, a$ and $a^{2}$. Moreover, the bounded and the spinal subgroups of $\overline{\ddot{\Gamma}}$ coincide.

There are refinements of Proposition 2.8.1, for example the following 
Proposition 2.8.6. Let $G \leq \operatorname{Aut}_{p} X^{*}$ be a self-similar, level-transitive group and suppose there is $v \in X^{n}$ and a function $f=[v]+\sum_{w \in S} w * f_{w}$ in $\mathcal{I}_{p}(G)$, where the $f_{w}$ are in $\mathcal{P}_{*}$ and $S$ is a subset of $X^{n+1} \backslash v X^{n}$. If $g \in \bar{G}$ is active at $u w$ with $w$ of length $n$, then $g$ is active at some vertex $u w^{\prime}$ with $w^{\prime}$ longer than $w$ and $w \nless w^{\prime}$.

Proof. Same as Proposition 2.8.1.

Corollary 2.8.7. With $G, v$ and $f$ as in the proposition, suppose $g \in \bar{G}$ is active at the vertex uw for some $u, w \in X^{*}$ with $w$ of length $n$. Then there is $w \neq w^{\prime} \in X^{n}$ so that $g @ u w^{\prime}$ is not finitary.

Proof. By the proposition, $g$ is active at some $v$ such that $u w^{\prime}<v$ with $w \neq w^{\prime} \in$ $X^{n}$. Therefore if $g @ u w^{\prime}$ would be finitary, it would have depth at least $n+1$, and this is impossible by Corollary 2.8.2.

Considered as an abstract group, an infinite profinite group is far from being torsion. For example, "almost any" $n$-tuple of elements in $\mathrm{Aut}_{p} X^{*}$ generates a free group of rank $n$, according to [Bha95]. Looking at the spinal subgroup of a closed subgroup of $\operatorname{Aut}_{p} X^{*}$, one might get torsion as the following shows. This applies in particular to the closure of the Grigorchuk group and of the GuptaSidki group.

Theorem 2.8.8. Let $G \leq \operatorname{Aut}_{p} X^{*}$ be a self-similar, level-transitive group and suppose there is $x \in X$ and a function $f=[x]+\sum_{w \in S} w * f_{w}$ in $\mathcal{I}_{p}(G)$, where the $f_{w}$ are in $\mathcal{P}_{*}$ and $S$ is a subset of $X^{2} \backslash x X$. If every element of the signature $\Sigma(G)$ has a zero entry, then every spinal element of $\bar{G}$ has finite order.

Proof. By Corollary 2.8.2, any finitary element in $\bar{G}$ has depth at most 1, and thus order at most $p$. We use induction to prove $\left(\mathcal{H}_{k}\right)$ : if $g \in \bar{G}$ has $(<k$ spines and $[\varnothing](g) \neq 0)$ or $(\leq k$ spines and $[\varnothing](g)=0)$, then $g$ has finite order.

First of all, $\left(\mathcal{H}_{1}\right)$ holds: a 0 -spinal element is finitary and therefore torsion. Also, a 1-spinal element of $\bar{G}$ which has $[\varnothing](g)=0$ has no activity along its spine by Corollary 2.8.7. Therefore it fixes the 1-neighbourhood of the spine, and has order $p$.

Now suppose $\left(\mathcal{H}_{k}\right)$ holds for some $k \geq 1$. Consider a $k$-spinal $g \in \bar{G}$ such that $[\varnothing](g) \neq 0$. By Lemma 2.8.9 there is $n$ so that $[\varnothing]\left(g^{p^{n}} @ v\right)=0$ and $g^{p^{n}} @ v$ is $k$-spinal for all $v \in X^{n}$. Therefore $g^{p^{n}} @ v$ has finite order for all $v \in X^{n}$ by the induction hypothesis, and so $g$ has finite order.

Consider now a $(k+1)$-spinal $g \in \bar{G}$ such that $[\varnothing](g)=0$. Let $S$ be the set of spines of $g$ and set $w$ be the maximal common prefix $S$. By Corollary 2.8.7, $[v](g)=0$ for all $v \in \operatorname{Prefix}(w)$. Therefore $g$ fixes the 1-neighbourhood $N_{1}(w)$ of $w$ and because of the choice of $w$, all the states of $g$ at $u \in N_{1}(w)$ are spinal elements with at most $k$ spines. By induction hypothesis they are all of finite order, and so is $g$.

Lemma 2.8.9. Let $G$ be a closed subgroup of Aut $_{p} X^{*}$ and suppose every element of the signature $\Sigma(G)$ has a zero entry. Then for all $g \in G$, there is an integer 


\subsection{Torsion Groups}

$n$ so that $[v]\left(g^{p^{n}}\right)=0$ for all $v \in X^{n}$. If moreover $g$ is $k$-spinal then $n$ can be chosen so that $g^{p^{n}} @ v$ is $k$-spinal for all $v \in X^{n}$.

Proof. First, notice that if $g \in \operatorname{Aut}_{p} X^{*}$ satisfies $[\varnothing](g) \neq 0$, then for all $x \in X$ and all $i \geq 1$ we have the equality

$$
\left[x * \Delta^{i-1}\right]\left(g^{p}\right)=\left[\Delta^{i}\right](g) .
$$

This is a direct consequence of Equations (1.7.1) and (2.2.1). By induction we then see that if $\left[\Delta^{0}\right](g), \ldots,\left[\Delta^{n-1}\right](g)$ are all non-zero, then for all $v \in X^{n}$ and all $i \geq n$ we have

$$
\left[v * \Delta^{i-n}\right]\left(g^{p^{n}}\right)=\left[\Delta^{i}\right](g)
$$

In particular we have $[v]\left(g^{p^{|v|}}\right)=\left[\Delta^{|v|}\right](g)$ for all $v$ of length $\leq n$.

Now by hypothesis there must be a zero entry in $\Sigma(g)$, say at position $n$. In other words, $\left[\Delta^{n}\right](g)=0$. We thus have $[v]\left(g^{p^{n}}\right)=0$ for all $v \in X^{n}$. It is then easy to see that $g$ induces a $p^{n}$-cycle on $X^{n}$, and the statement for $k$-spinal elements follows.

Example 2.8.10. The Grigorchuk group $\mathrm{G}$ (see Section A.1) satisfies $P_{0}(\mathrm{G})=0$ with $P_{0}=\left[\underline{0}+\underline{1} *\left(\Delta^{1}+\Delta^{2}\right)\right]$. Therefore the spinal subgroup of $\overline{\mathrm{G}}$ is torsion. The same applies to the spinal subgroup of $\overline{\mathrm{G}_{2}}=\mathcal{V}\left(X^{*} *\left\{P_{0}, P_{0}^{a}\right\}\right)$ which we study in Chapter 7.

Example 2.8.11. The Gupta-Sidki group $\ddot{\Gamma}$ (see Section A.3) satisfies $[-\underline{0}+$ $\left.\Delta_{11}\right](\ddot{\Gamma})=0$. Therefore the spinal subgroup of $\ddot{\vec{\Gamma}}$ is torsion.

Example 2.8.12. The group $B$ defined in Section A.5 satisfies $\left[-\underline{0}-\Delta_{02}+\right.$ $\left.\Delta_{022}\right](B)=0$. Therefore the spinal subgroup of $\bar{B}$ is torsion. 


\section{Chapter 3}

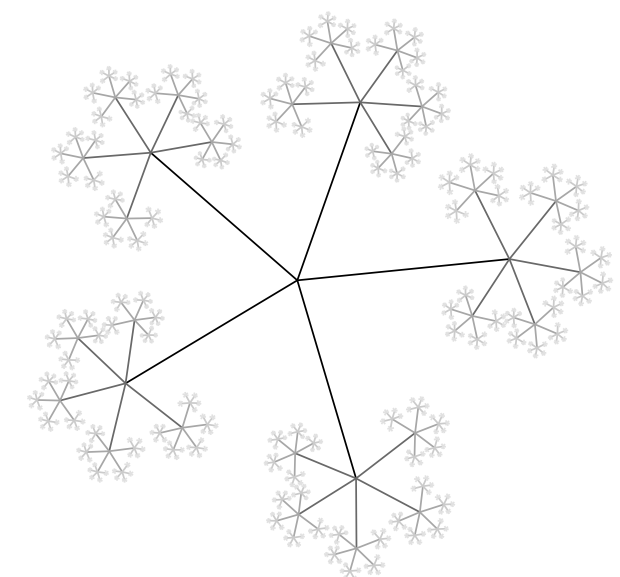

\section{Wreath Product of Affine Group Schemes}

Recall the wreath product $G \imath_{S} H$ of the groups $G$ and $H$, with $H$ acting on the set $S$, is defined as follows. We consider the set $G^{S}$ of all functions from $S$ to $G$ : this is a group under pointwise operation. Then $H$ acts on $G^{S}$ (by translation on coordinates), and the semidirect product $G^{S} \rtimes H$ corresponding to this action is, by definition, the wreath product $G ?_{S} H$.

In this chapter we introduce the wreath product of affine group schemes. Affine group schemes can be defined as $\operatorname{Spec} A$, where $A$ is a Hopf algebra. More precisely, to any commutative Hopf algebra $A$, one associates the affine group scheme $S=\operatorname{Spec} A$. This is the functor $\operatorname{Hom}_{k}(A, \bullet)$ which associates to any $k$-algebra $R$ the set $S(R)=\operatorname{Hom}_{k}(A, R)$ of $k$-algebra morphisms from $A$ to $R$. The elements of $S(R)$ are called the points of $S$ on $R$. They form a group under "convolution".

In principle, anything that holds for groups also holds for Hopf algebras. Loosely speaking, we just need to "add co-". In particular, corresponding to the product of a group, any Hopf algebra has a co-product. One thing to keep in mind is that the dual of the diagonal map $G \rightarrow G \times G$ is the multiplication $A \otimes A \rightarrow A$ in the algebra.

We define the wreath product of group schemes on the Hopf algebra side, introducing the co-wreath product of Hopf algebras. For this definition, we just rewrite the usual definition of wreath product of groups, and "reverse arrows".

In Sections 3.1, 3.2 and 3.3, we give the relevant definitions and build the wreath product of affine group schemes, step by step. In Sections 3.4 and 3.5, we prove some results which relate iterated wreath products of group schemes to the automorphism group of a rooted tree. In particular, we prove that the Lie algebra of the iterated wreath product of the Frobenius kernel is isomorphic to the Lie algebra obtained from the lower central series of $\operatorname{Aut}_{p} X^{n}$. 


\subsection{Affine Schemes}

\section{A Few Words About Hopf Algebras}

There are many books about affine algebraic groups, affine group schemes and Hopf algebras. The difficulty here is to grasp the concepts behind all this huge theory, in spite of the variety of viewpoints developed in the literature. Thus the books devoted to algebraic groups and group schemes (e.g. [Bor69, Hoc71, Wat79, Hoc81]) dwell rather quickly over Hopf algebras, concentrating on geometric matters. But still, they go all the way to the Lie algebra structure on the tangent space at the identity in terms of differentiations and of derivations (this is especially true for [Bor69, Wat79], where the restricted Lie algebra structure is made explicit). On the other side, the classical references about Hopf algebras [MM65, Swe69] concentrate on the Hopf algebras themselves, without talking about the corresponding affine group schemes they represent.

Roughly speaking, a Hopf algebra is a vector space $A$ together with five structural maps which fit together in a suitable way. These maps are: a unit $u_{A}$, a product $\mu_{A}$, a co-unit $\varepsilon_{A}$ (also called augmentation), a co-product $\delta_{A}$ and an antipode $\eta_{A}$. In particular, $\left(A, u_{A}, \mu_{A}\right)$ is an ordinary algebra. Dually, $\left(A, \varepsilon_{A}, \delta_{A}\right)$ is a co-algebra. The co-product and the augmentation are required to be algebra morphisms. This is equivalent to requiring the unit and product to be co-algebra morphisms. This is about all we shall need, and the reader is encouraged to consult the references.

\subsection{Affine Schemes}

We follow the approach of Waterhouse [Wat79], which fits very well in the gap between "classical" group theory, "classical" algebraic geometry, and the more modern approach of Grothendieck and others [Gro60].

We fix a ring $k$ (commutative, with unit). Unless otherwise stated, the algebras we consider are all over $k$, and they are commutative, with unit. Likewise, Hopf algebras shall all be commutative.

An affine scheme over $k$ is a representable functor from $k$-algebras to sets. Given an affine scheme $G$ and a $k$-algebra $R$, one can consider its $R$-points $G(R)$. This is just a set, and it is functorial in $R$. The fact that $G$ be representable means by definition that there is a $k$-algebra $A$ so that $G(R)$ is the set $\operatorname{Hom}_{k}(A, R)$ of $k$ algebra morphisms $A \rightarrow R$. Morphisms of affine schemes are natural transformations between the corresponding functors. By Yoneda's lemma, they correspond (contravariantly) to algebra morphisms.

An affine group scheme over $k$ is a representable functor from $k$-algebras to groups. If an algebra $A$ represents a group scheme $G$, then $A$ has the structure of a Hopf algebra. Morphisms of affine group schemes are natural transformations which preserve the group structure. They correspond to Hopf algebra morphisms.

Since we are only concerned with affine group schemes, we shall almost always work on the Hopf algebra side of the problem, avoiding the technicalities of scheme theory. The price we pay is that geometric considerations are relayed to the background. 


\subsubsection{Finite Constant Schemes}

Let $S$ be a finite set. The set of functions $K^{S}$ from $S$ to $k$ becomes a $k$-algebra when we consider pointwise addition an multiplication. This algebra represents the constant scheme for $S$. We denote it by $\underline{S}$.

For each element $s \in S$, consider the map

$$
\begin{aligned}
e_{s}: S & \rightarrow k, \\
t & \mapsto \begin{cases}1 & \text { if } s=t, \\
0 & \text { otherwise }\end{cases}
\end{aligned}
$$

The elements $e_{s}$ span $k^{S}$. They are idempotents: $e_{s}^{2}=e_{s}$, and the product $e_{s} e_{t}$ is equal to 0 if $s \neq t$. Therefore, if $R$ is a $k$-algebra with no idempotents except 0 and 1 , the algebra morphisms $\operatorname{Hom}_{k}\left(k^{S}, R\right)$ correspond bijectively to the elements of $S$. Indeed, such a morphism must send exactly one $e_{s}$ to 1 , and all the others to 0 . Thus the points $\underline{S}(R)$ of the scheme $\underline{S}$ over $R$ are in bijection with the set $S$.

If $G$ is a finite group, then $k^{G}$ becomes a Hopf algebra with the maps

$$
\begin{aligned}
& \varepsilon: k^{G} \rightarrow k, \quad \delta: k^{G} \rightarrow k^{G} \otimes k^{G}, \quad \eta: k^{G} \rightarrow k^{G}, \\
& e_{g} \mapsto\left\{\begin{array}{ll}
1 & \text { if } g=1, \\
0 & \text { otherwise, }
\end{array} \quad e_{g} \mapsto \sum_{\substack{h, h^{\prime} \in G \\
h h^{\prime}=g}} e_{h} \otimes e_{h^{\prime}}, \quad e_{g} \mapsto e_{g^{-1}} .\right.
\end{aligned}
$$

Here again, if $R$ is a $k$-algebra with no idempotents except 0 and 1 , the points $\underline{G}(R)$ form a group isomorphic to $G$. If $k$ is a field, then the $k$-dual of $k^{G}$ is the group algebra $k G$, endowed with the co-product $\delta: g \mapsto g \otimes g$. The Hopf algebra $k G$ is co-commutative; it is commutative if and only if $G$ is Abelian.

\subsubsection{The Frobenius Kernel}

Consider the polynomial algebra $k[X]$. Endowed with the algebra morphisms

$$
\begin{aligned}
\varepsilon: k[X] & \rightarrow k, & \delta: k[X] & \rightarrow k[X] \otimes k[X], & \eta: k[X] & \rightarrow k[X], \\
X & \mapsto 0, & X & \mapsto X \otimes 1+1 \otimes X, & X & \mapsto-X,
\end{aligned}
$$

$k[X]$ becomes a Hopf algebra, representing the additive group $\mathbb{G}_{a}$. If $p \cdot 1=0$ in $k$ for a prime number $p$, then we have $\delta\left(X^{p}\right)=X^{p} \otimes 1+1 \otimes X^{p}$, and the ideal generated by $X^{p}$ is a Hopf ideal. Thus the quotient $k[X] /\left(X^{p}\right)$ inherits the Hopf algebra structure of $k[X]$, and represents a closed subscheme of $\mathbb{G}_{a}$. It is called the Frobenius kernel, and denoted by $\boldsymbol{\alpha}_{p}$.

The scheme $\boldsymbol{\alpha}_{p}$ is infinitesimal: the augmentation ideal $(X)$ is nilpotent. Consequently, $\boldsymbol{\alpha}_{p}$ has only one point on any field $R$. On the other hand, $\boldsymbol{\alpha}_{p}$ is not isomorphic to the trivial scheme Spec $k$. For example, the map $\operatorname{Lie}\left(\boldsymbol{\alpha}_{p}\right) \rightarrow \operatorname{Lie}\left(\mathbb{G}_{a}\right)$ induced by $\boldsymbol{\alpha}_{p} \rightarrow \mathbb{G}_{a}$ is an isomorphism. 


\subsection{Exponential Objects}

We consider a category $\mathcal{C}$ with products, and two objects $S$ and $T$ in $\mathcal{C}$.

Definition 3.2.1. An object $S^{T}$ with a morphism $\phi: T \times S^{T} \rightarrow S$ is called an exponential of $S$ by $T$ if the following universal property holds: for any object $U \in \mathcal{C}$, and any morphism $f: T \times U \rightarrow S$, there is a unique morphism $\bar{f}: U \rightarrow S^{T}$ so that the diagram

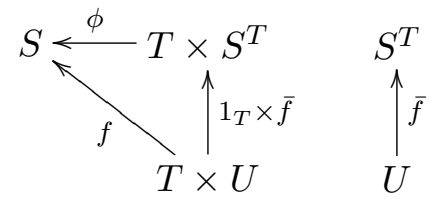

is commutative.

If the exponential of $S$ by $T$ exists, then it is unique up to unique isomorphism.

Example 3.2.2. In the category of sets, $S^{T}$ is the set of all maps $T \rightarrow S$, and $\phi$ is the evaluation: $(t, f) \mapsto \phi(t, f)=f(t)$.

We shall be interested in affine schemes, so we state the dual definition for algebras. Consider two algebras $A$ and $B$.

Definition 3.2.3. An algebra $A^{B}$ with a morphism

$$
\varphi: A \rightarrow B \otimes A^{B}
$$

is called a co-exponential of $A$ by $B$, if for any algebra $R$ and morphism $f: A \rightarrow$ $B \otimes R$, there is a unique morphism $\bar{f}: A^{B} \rightarrow R$ so that the diagram

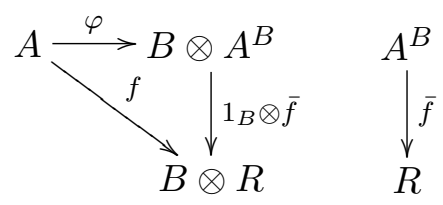

is commutative.

If the co-exponential of $A$ by $B$ exists, then it is unique up to unique isomorphism. We call the morphism $\varphi$ the co-evaluation of $A^{B}$.

The correspondence between affine schemes and algebras yields the following. If $S$ and $T$ are affine schemes represented by $A$ and $B$ respectively, and if $A^{B}$ exists, then $S^{T}$ exists and is represented by $A^{B}$.

Proposition 3.2.4. Let $S$ and $T$ be finite sets, and let $A$ and $B$ represent the constant schemes $\underline{S}$ and $\underline{T}$ respectively. The algebra representing $\underline{S^{T}}$ is isomorphic to $A^{B}$. In particular $A^{B}$ exists, and the equality $\underline{S}^{\underline{T}}=\underline{S^{T}}$ holds. 
Proof. Let $\left\{e_{s} \mid s \in S\right\}$ and $\left\{e_{t} \mid t \in T\right\}$ be the standard bases of $A$ and $B$. Let $C$ be the algebra representing $\underline{S^{T}}$, and let $e_{h}$ be the standard basis of $C$, where $h$ ranges over $S^{T}$. If $h$ is a function $\operatorname{Dom}(h) \rightarrow S$ which is defined on $\operatorname{Dom}(h) \subseteq T$, we set

$$
e_{h}=\sum_{\substack{h^{\prime} \in S^{T} \\ h=\left.h^{\prime}\right|_{\operatorname{Dom}(h)}}} e_{h^{\prime}}
$$

One can check that the product of $e_{h}$ and $e_{h^{\prime}}$ is non-zero if and only if $h$ and $h^{\prime}$ coincide on $\operatorname{Dom}(h) \cap \operatorname{Dom}\left(h^{\prime}\right)$. In this case, we have $e_{h} e_{h^{\prime}}=e_{h^{\prime \prime}}$, where $h^{\prime \prime}$ is the function defined on $\operatorname{Dom}(h) \cup \operatorname{Dom}\left(h^{\prime}\right)$ which coincides with $h$ on $\operatorname{Dom}(h)$, and with $h^{\prime}$ on $\operatorname{Dom}\left(h^{\prime}\right)$. We set

$$
\begin{aligned}
\varphi: A & \rightarrow B \otimes C \\
e_{s} & \mapsto \sum_{t \in T} e_{t} \otimes e_{t \mapsto s} .
\end{aligned}
$$

where $e_{t \mapsto s}$ is the element $e_{h}$ for $h:\{t\} \rightarrow S$ defined by $h(t)=s$. We prove that $(C, \varphi)$ satisfies the universal property of exponential objects.

Let $f: A \rightarrow B \otimes R$ be an algebra morphism, and write $f\left(e_{s}\right)=\sum_{t \in T} e_{t} \otimes r_{t s}$. We define the linear map $\bar{f}: C \rightarrow R$ by $\bar{f}\left(e_{h}\right)=\prod_{t \in \operatorname{Dom}(h)} r_{t h(t)}$. Because $f$ is an algebra morphism, we have $r_{t s} r_{t s^{\prime}}=0$ when $s \neq s^{\prime}$, and $\left(r_{t s}\right)^{2}=r_{t s}$. It is then easy to see that $\bar{f}$ is an algebra morphism, and that the composite $(1 \otimes \bar{f}) \circ \varphi$ is equal to $f$.

Finally, any morphism $\tilde{f}$ which satisfies $(1 \otimes \tilde{f}) \circ \varphi$ must send $e_{t \mapsto s}$ to $r_{t s}$. Since the elements $e_{t \mapsto s}$ generate $C$, the map $\bar{f}$ is unique.

Proposition 3.2.5. Let $k$ be a field with $\operatorname{char}(k)=p>0$, and consider the algebras $A=k\left[X_{1}, \ldots, X_{n}\right] /\left(\left\{X_{i}^{p} \mid i \in I\right\}\right)$ with $I \subseteq\{1, \ldots, n\}$, and $B=k[Y] /\left(Y^{p}\right)$. The co-exponential of $A$ by $B$ is

$$
A^{B}=k\left[X_{0,1}, \ldots, X_{p-1,1}, \ldots, X_{0, n}, \ldots, X_{p-1, n}\right] /\left(\left\{X_{0, i}^{p} \mid i \in I\right\}\right)
$$

with the co-evaluation $\varphi: X_{i} \mapsto \sum_{m=0}^{p-1} Y^{m} \otimes X_{m, i}$.

Proof. Let $C$ be the algebra $k\left[X_{0,1}, \ldots, X_{p-1,1}, \ldots, X_{0, n}, \ldots, X_{p-1, n}\right] /\left(\left\{X_{0, i}^{p} \mid i \in\right.\right.$ $I\})$. We check the universal property for $(C, \varphi)$.

First, $\varphi$ is an algebra morphism. Indeed, we only have to check

$$
\varphi\left(X_{i}\right)^{p}=\left(\sum_{m=0}^{p-1} Y^{m} \otimes X_{m, i}\right)^{p}=\sum_{m=0}^{p-1}\left(Y^{m} \otimes X_{m, i}\right)^{p}=\sum_{m=0}^{p-1} Y^{p m} \otimes\left(X_{m, i}\right)^{p}=0
$$

for all $i \in I$. Next, let $f: A \rightarrow B \otimes R$ be an algebra morphism, and write $f\left(X_{i}\right)=\sum_{m=0}^{p-1} Y^{m} \otimes a_{m, i}$. We define $\bar{f}: C \rightarrow R$ by $X_{m, i} \mapsto a_{m, i}$. Since $f$ is an algebra morphism, we have $f\left(X_{i}^{p}\right)=f\left(X_{i}\right)^{p}=\left(\sum_{m=0}^{p-1} Y^{m} \otimes a_{m, i}\right)^{p}=$ $\sum_{m=0}^{p-1} Y^{p m} \otimes\left(a_{m, i}\right)^{p}$. Therefore $\left(a_{0, i}\right)^{p}=0$ for all $i \in I$. Thus the map $\bar{f}$ extends 


\subsection{Exponential Objects}

to an algebra morphism. It is readily checked that the composition $(1 \otimes \bar{f}) \circ \varphi$ is equal to $f$.

If $\tilde{f}$ is another map satisfying $(1 \otimes \tilde{f}) \circ \varphi=f$, then $\tilde{f}$ must send $X_{m, i}$ to $a_{m, i}$, and therefore $\tilde{f}=\bar{f}$ is unique.

\subsubsection{Additional Structure}

If $G$ is a group and $S$ is a set, then $G^{S}$ becomes a group under pointwise operation. We work out the dual construction.

Let $A$ be a Hopf algebra and $B$ an algebra. Then $A^{B}$ inherits a Hopf algebra structure as follows. The compositions

$$
\begin{aligned}
& A \stackrel{\varepsilon_{A}}{\longrightarrow} k \stackrel{u_{B}}{\longrightarrow} B=B \otimes k, \\
& A \stackrel{\eta_{A}}{\longrightarrow} A \stackrel{\varphi}{\longrightarrow} B \otimes A^{B}, \\
& A \stackrel{\delta_{A}}{\longrightarrow} A \otimes A \stackrel{\varphi \otimes \varphi}{\longrightarrow} B \otimes A^{B} \otimes B \otimes A^{B} \\
& \quad \stackrel{1 \otimes T \otimes 1}{\longrightarrow} B \otimes B \otimes A^{B} \otimes A^{B} \stackrel{\mu_{B} \otimes 1 \otimes 1}{\longrightarrow} B \otimes A^{B} \otimes A^{B} .
\end{aligned}
$$

are all algebra morphisms (remember $A$ is commutative, therefore $\eta_{A}$ is a morphism of algebras), and by the universal property of co-exponential objects, they correspond to algebra morphisms

$$
\begin{aligned}
& \varepsilon: A^{B} \rightarrow k, \\
& \eta: A^{B} \rightarrow A^{B}, \\
& \delta: A^{B} \rightarrow A^{B} \otimes A^{B} .
\end{aligned}
$$

One can check that this turns $A^{B}$ into a Hopf algebra.

Proposition 3.2.6. Let $G$ be a finite group and $S$ a finite set. Let $A$ be the Hopf algebra representing the constant scheme $\underline{G}$ and let $B$ be the algebra representing the constant scheme $\underline{S}$. Then the Hopf algebra $A^{B}$ represents the constant group scheme $\underline{G^{S}}$ corresponding to the finite group $G^{S}$.

Proof. Let $C$ be the Hopf algebra representing $G^{S}$. By Proposition 3.2.4, we know that the algebras $A^{B}$ and $C$ are isomorphic. Thus we only need to check that the co-product of $C$ coincides with that of $A^{B}$. We check this for the generators $e_{s \mapsto g}$ of $A^{B}$ (see Proposition 3.2 .4 for the notation).

Recall the co-evaluation of $A^{B}$ is given by $e_{g} \mapsto \sum_{s \in S} e_{s} \otimes e_{s \mapsto g}$. We then compute the composite $\left(\mu_{B} \otimes 1 \otimes 1\right) \circ(1 \otimes T \otimes 1) \circ(\varphi \otimes \varphi) \circ \delta_{A}$ :

$$
e_{g} \mapsto \sum_{\substack{s \in S \\ g^{\prime}, g^{\prime \prime} \in G \\ g^{\prime} g^{\prime \prime}=g}} e_{s} \otimes e_{s \mapsto g^{\prime}} \otimes e_{s \mapsto g^{\prime \prime}} .
$$

Thus the co-product of $A^{B}$ is given by

$$
e_{s \mapsto g} \mapsto \sum_{\substack{g^{\prime}, g^{\prime \prime} \in G \\ g^{\prime} g^{\prime \prime}=g}} e_{s \mapsto g^{\prime}} \otimes e_{S \mapsto g^{\prime \prime}} .
$$

This agrees with the co-product of $C$. 


\subsection{Some Hopf Algebra Constructions}

\subsubsection{Co-Semidirect Product}

Let $B$ be a Hopf algebra. A left $B$-comodule algebra is an algebra $A$ together with an algebra morphism

$$
\rho: A \rightarrow B \otimes A
$$

which turns $A$ into a left $B$-comodule.

Consider two Hopf algebras $A$ and $B$, and suppose $A$ is a $B$-comodule algebra with structure map $\rho: A \rightarrow B \otimes A$. The co-semidirect product of $A$ by $B$ (along $\rho$ ), written $A \rtimes^{\circ} B$ is the Hopf algebra defined as follows. The underlying algebra of $A \rtimes^{\circ} B$ is the algebra $A \otimes B$. The co-product of $A \rtimes^{\circ} B$ is given by the composition

$$
\begin{aligned}
& A \otimes B \stackrel{\delta_{A} \otimes \delta_{B}}{\longrightarrow} A \otimes A \otimes B \otimes B \\
& \stackrel{1 \otimes 1 \otimes \rho \otimes 1}{\longrightarrow} A \otimes B \otimes B \otimes 1 \\
& \stackrel{1 \otimes \otimes}{\longrightarrow} A \otimes B \otimes A \otimes B \\
& \stackrel{1 \otimes \mu_{B} \otimes 1 \otimes 1}{\longrightarrow} A \otimes B \otimes A \otimes B .
\end{aligned}
$$

One can check that this map is co-associative, and that $\varepsilon=\varepsilon_{A} \otimes \varepsilon_{B}$ is a co-unit. We define $\eta: A \rtimes^{\circ} B \rightarrow A \rtimes^{\circ} B$ as the composition

$$
\begin{aligned}
A \otimes B & \stackrel{\rho \otimes 1}{\longrightarrow} B \otimes A \otimes B \stackrel{T \otimes 1}{\longrightarrow} A \otimes B \otimes B \\
& \stackrel{\eta_{A} \otimes \eta_{B} \otimes \eta_{B}}{\longrightarrow} A \otimes B \otimes B \stackrel{1 \otimes \mu_{B}}{\longrightarrow} A \otimes B .
\end{aligned}
$$

Again, one can check that this is an antipode for $A \rtimes^{\circ} B$.

Consider affine group schemes $G$ and $H$ represented by $A$ and $B$. Suppose $H$ acts on $G$ (i.e. $H(R)$ acts on $G(R)$, functorially in $R$ ). Then $A$ is a $B$-comodule algebra, and the semidirect product of $G$ and $H$ is the group scheme $G \rtimes H$ represented by $A \rtimes^{\circ} B$.

Proposition 3.3.1. Let $G$ and $H$ be finite groups, and suppose $H$ acts on $G$. Let $A$ and $B$ be the Hopf algebras representing the constant schemes $\underline{G}$ and $\underline{H}$ respectively. The action of $H$ on $G$ induces a co-action of $B$ on $A$, and the cosemidirect product $A \rtimes^{\circ} B$ represents the constant scheme $\underline{G \rtimes H}$ corresponding

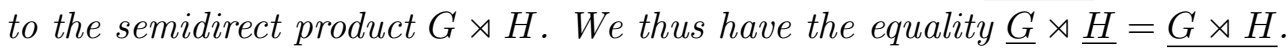

Proof. Let $C$ be the algebra representing $\underline{G \rtimes H}$. The action of $H$ on $G$ induces the co-action

$$
\begin{aligned}
A & \rightarrow B \otimes A, \\
e_{g} & \mapsto \sum_{h \in H} e_{h} \otimes e_{h^{-1}(g)} .
\end{aligned}
$$

We only need to check that the co-product of $A \rtimes^{\circ} B$ is the co-product of $C$. We compute the co-product of $A \rtimes^{\circ} B$ :

$$
e_{g} \otimes e_{h} \mapsto \sum_{\substack{g^{\prime}, g^{\prime \prime} \in G \\ g^{\prime} g^{\prime \prime}=g \\ h^{\prime}, h^{\prime \prime} \in H \\ h^{\prime} h^{\prime \prime}=h}} e_{g^{\prime}} \otimes e_{h^{\prime}} \otimes e_{\left(h^{\prime}\right)^{-1}\left(g^{\prime \prime}\right)} \otimes e_{h^{\prime \prime}}
$$




\subsection{Some Hopf Algebra Constructions}

A change of variables in the summation yields

$$
e_{g} \otimes e_{h} \mapsto \sum_{\begin{array}{c}
g^{\prime}, g^{\prime \prime} \in G \\
g^{\prime} \cdot h^{\prime}\left(g^{\prime \prime}\right)=g \\
h^{\prime}, h^{\prime \prime} \in H \\
h^{\prime} h^{\prime \prime}=h
\end{array}} e_{g^{\prime}} \otimes e_{h^{\prime}} \otimes e_{g^{\prime \prime}} \otimes e_{h^{\prime \prime}}
$$

This is the co-product of $C$.

\subsubsection{Co-Wreath Product}

Consider a group $H$ and two sets $S$ and $T$. If $H$ acts on $T$, there is an action of $H$ on $S^{T}$ defined as

$$
\left(f^{h}\right)(t)=f(h(t)) .
$$

Here is the dual statement for algebras:

Lemma 3.3.2. Let $B$ be a Hopf algebra, and $M$ and $N$ be algebras; suppose $N$ is a right $B$-comodule algebra under

$$
\rho: N \rightarrow N \otimes B
$$

and suppose $M^{N}$ exists. Then there is a natural map

$$
\psi_{B}^{*}: M^{N} \rightarrow B \otimes M^{N}
$$

which turns $M^{N}$ into a left B-comodule algebra.

Proof. Composing the co-evaluation $\varphi: M \rightarrow N \otimes M^{N}$ with $\rho \otimes 1$, we get an algebra morphism

$$
(\rho \otimes 1) \circ \varphi: M \rightarrow N \otimes B \otimes M^{N} .
$$

By the universal property of co-exponential objects, we have a commutative diagram

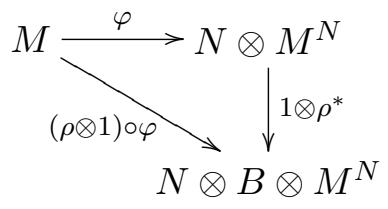

The above defines $\rho^{*}: M^{N} \rightarrow B \otimes M^{N}$. Next, we have a commutative diagram

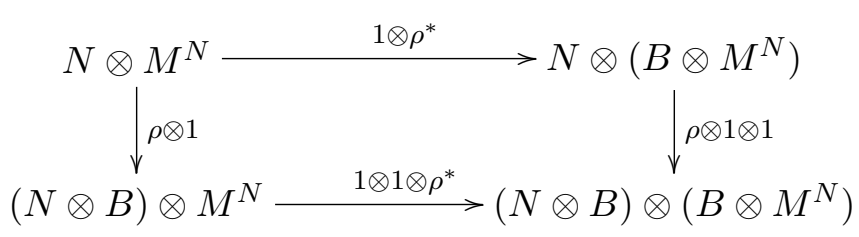

By Diagram (3.3.1), the lower left and the upper right corner are related by the identity map. Since $N$ is a right $B$-comodule algebra, we then easily see that $M^{N}$ is a left $B$-comodule algebra. 
Consider two Hopf algebras $A$ and $B$, and an algebra $M$. Suppose $M$ is a right $B$-comodule algebra with structure map $\rho: M \rightarrow M \otimes B$ and suppose $A^{M}$ exists. The co-wreath product of $A$ by $B$ (co-acting on $M$ ), written $A 2_{M}^{\circ} B$ or $A \imath^{\circ} B$, is the co-semidirect product

$$
A \imath^{\circ} B=A^{M} \rtimes^{\circ} B
$$

with the natural co-action of $B$ on $A^{M}$ given by Lemma 3.3.2.

Let $G$ and $H$ be affine group schemes represented by $A$ and $B$ respectively, and let $S$ be an affine scheme represented by $M$. Suppose $H$ acts on $S$. The wreath product of $G$ by $H$ (acting on $S$ ), written $G \imath_{S} H$ or $G \imath H$, is the group scheme represented by $A 2_{M}^{\circ} B$, if this Hopf algebra exists.

Proposition 3.3.3. Let $G$ and $H$ be finite groups, and suppose $H$ acts on the finite set $S$. Let $A, B$ and $M$ represent the constant schemes $\underline{G}, \underline{H}$ and $\underline{M}$ respectively. Then $A$ < $B$ represents the constant scheme $G$ ? $H$. In particular the equality $\underline{G} \imath \underline{H}=\underline{G\urcorner H}$ holds.

Proof. This is a consequence of Propositions 3.2.4, 3.2.6 and 3.3.1.

\subsection{Group Schemes Corresponding to Aut $X^{*}$}

In this section we consider $k$ a finite field, for comparison with Chapters 1 and 2 . Let $X$ be a finite set and let $\mathcal{A}_{1}$ be the Hopf algebra representing the constant scheme $G_{1}=\operatorname{Sym}(X)$. Write $G_{n}=\left(\cdots\left(G_{1} \succ G_{1}\right) \ldots\right) \curlywedge G_{1}$ and let $A_{n}=\left(\cdots\left(A_{1} \curlywedge^{\circ}\right.\right.$ $\left.\left.A_{1}\right) \ldots\right) \imath^{\circ} A_{1}$ be the algebra representing $G_{n}$.

Proposition 3.4.1. For all $n \geq 0$, the Hopf algebra $A_{n}$ is isomorphic to the Hopf algebra $\mathcal{A}_{n}$ from Section 1.1 .

Proof. The claim holds for $n=0$, and the induction step holds by Proposition 3.3.3.

Corollary 3.4.2. For all $n \geq 0$, if $R$ is a k-algebra with no idempotents except 0 and 1 , the group $G_{n}(R)$ is isomorphic to Aut $X^{n}$.

Consider a prime number $p$ and $k=\mathbb{F}_{p}$. Let $P_{1}$ be the Hopf algebra representing the constant group scheme $W_{1}=\mathbb{Z} / p \mathbb{Z}$. Write $W_{n}=\left(\cdots\left(W_{1} \prec W_{1}\right) \ldots\right)$ 々 $W_{1}$ and let $P_{n}=\left(\cdots\left(P_{1} \curlywedge^{\circ} P_{1}\right) \ldots\right) \imath^{\circ} P_{1}$ be the algebra representing $P_{n}$.

Proposition 3.4.3. For all $n \geq 0$, the Hopf algebra $P_{n}$ is isomorphic to the Hopf algebra $\mathcal{P}_{n}$ from Section 2.1 .

Proof. The claim holds for $n=0$, and the induction step holds by Proposition 3.3.3.

Corollary 3.4.4. For all $n \geq 0$, if $R$ is an $\mathbb{F}_{p}$-algebra with no idempotents except 0 and 1 , the group $W_{n}(R)$ is isomorphic to $\operatorname{Aut}_{p} X^{n}$. 


\subsection{Iterated Wreath Product of the Frobenius Kernel}

\subsection{Iterated Wreath Product of the Frobenius Kernel}

Let $\boldsymbol{\omega}_{n}$ be the wreath product $\left(\cdots\left(\boldsymbol{\alpha}_{p} \succ \boldsymbol{\alpha}_{p}\right) \ldots\right)$ 乙 $\boldsymbol{\alpha}_{p}$ with $n$ factors $\boldsymbol{\alpha}_{p}$. Let Lie $\left(\operatorname{Aut}_{p} X^{n}\right)$ be the Lie algebra coming from the quotients of the lower central series of $\operatorname{Aut}_{p} X^{n}$, as in Section 4.2 (see also Theorem 4.2.1).

Theorem 3.5.1. For any $\mathbb{F}_{p}$-algebra $k$ and for all $n \geq 0$, the Lie algebras $\operatorname{Lie}\left(\boldsymbol{\omega}_{n}\right)$ and Lie $\left(\operatorname{Aut}_{p} X^{n}\right) \otimes_{\mathbb{F}_{p}} k$ are isomorphic.

Before proving this theorem, we need some notation and a proposition. For this section, we set $X=\{0, \ldots, p-1\}$. For a word $w \in X^{*}$, let $w_{i}$ denote its $i$-th letter. We let $X^{<n}$ denote the union $X^{0} \cup \cdots \cup X^{n-1}$. For an element $w \in X^{*}$ of length at least $n$, we let $\pi_{n}(w)$ denote the prefix of $w$ of length $n$, that is: $\pi_{n}(w)=w_{1} \cdots w_{n}$. In particular, $\pi_{0}(w)$ is always the empty word.

We introduce a partial order on $X^{*}$, different from the prefix-order. For two words $v, w \in X^{*}$ of the same length, we write $v \preceq w$ if $v_{i} \leq w_{i}$ for all $1 \leq i \leq|w|$. If we have $v \preceq w$, then the difference $w-v \in X^{*}$ is computed componentwise.

Let $F_{n}$ be the Hopf algebra representing $\boldsymbol{\omega}_{n}$.

Proposition 3.5.2. As an algebra, $F_{n}$ is $k\left[X_{w}\right] /\left(X_{v}^{p}\right)$, with $w \in X^{<n}$ and $v$ ranges over the words $\varnothing, 0, \ldots, 0^{n-1}$. Moreover, the co-product of $F_{n}$ is given by

$$
\begin{aligned}
X_{w} \mapsto & X_{w} \otimes 1+1 \otimes X_{w} \\
& +\sum_{d=0}^{|w|-1}\left(w_{d+1}+1\right) \sum_{0^{d} \preceq i \preceq \pi_{d}(w)} X_{i} \otimes X_{\left(\pi_{d}(w)-i\right)\left(w_{d+1}+1\right) w_{d+2} \cdots w_{|w|}} .
\end{aligned}
$$

up to degree 2 and higher.

Proof. We proceed by induction. For $n=0$, the set $X^{<n}$ is empty, and $F_{0}=k$. We suppose the claim holds for some $n$, and show the induction step.

We construct the co-wreath product of $F_{n}$ by $F_{1}$. First, the co-exponential $F_{n}^{F_{1}}$ is the algebra $k\left[X_{w}\right] /\left(X_{v}^{p}\right)$, with $w \in X^{<n} \backslash\{\varnothing\}$ and $v$ ranges over $0, \ldots, 0^{n}$. As an algebra, $F_{n+1}$ is isomorphic to $F_{n}^{F_{1}} \otimes F_{1}$. Writing $F_{1}=k\left[X_{\varnothing}\right] /\left(X_{\varnothing}\right)^{p}$, the algebra $F_{n}^{F_{1}} \otimes F_{1}$ is as in the claim.

To compute the co-product of $F_{n+1}$, note that the co-evaluation $F_{n} \mapsto F_{1} \otimes$ $F_{n}^{F_{1}}$ is given by

$$
X_{w} \mapsto \sum_{m=0}^{p-1}\left(X_{\varnothing}\right)^{m} \otimes X_{m, w} .
$$

Next, $F_{1}$ co-acts on itself via its co-product:

$$
X_{\varnothing} \mapsto X_{\varnothing} \otimes 1+1 \otimes X_{\varnothing} .
$$

Thus in $F_{n+1}$, the co-product of $X_{\varnothing}$ is $X_{\varnothing} \otimes 1+1 \otimes X_{\varnothing}$. For the other variables, we first compute the co-product of $X_{m, w}$ in $F_{n}^{F_{1}}$ :

$$
\begin{aligned}
X_{m, w} \mapsto & X_{m, w} \otimes 1+1 \otimes X_{m, w} \\
& +\sum_{d=0}^{|w|-1}\left(w_{d+1}+1\right) \sum_{\substack{0 \leq i_{0} \leq m \\
0^{d} \preceq i \preceq \pi_{d}(w)}} X_{i_{0} i} \otimes X_{\left(m-i_{0}\right)\left(\pi_{d}(w)-i\right)\left(w_{d+1}+1\right) w_{d+2} \cdots w_{|w|}} .
\end{aligned}
$$


Applying Lemma 3.3 .2 , the co-action of $F_{1}$ on $F_{n}^{F_{1}}$ is given by

$$
X_{m, w} \mapsto \sum_{i=m}^{p-1}\left(\begin{array}{c}
i \\
m
\end{array}\right)\left(X_{\varnothing}\right)^{i-m} \otimes X_{i, w} \equiv 1 \otimes X_{m, w}+(m+1) X_{\varnothing} \otimes X_{(m+1) w}
$$

up to degree 2. Thus in $F_{n} 2^{\circ} F_{1}$, the co-product of $X_{m, w}$ is

$$
\begin{aligned}
X_{m, w} \mapsto & X_{m, w} \otimes 1+1 \otimes X_{m, w}+(m+1) X_{\varnothing} \otimes X_{(m+1), w} \\
& +\sum_{d=0}^{|w|-1}\left(w_{d+1}+1\right) \sum_{\substack{0 \leq i_{0} \leq m \\
0^{d} \preceq i \preceq \pi_{d}(w)}} X_{i_{0} i} \otimes X_{\left(m-i_{0}\right)\left(\pi_{d}(w)-i\right)\left(w_{d+1}+1\right) w_{d+2} \cdots w_{|w|}},
\end{aligned}
$$

neglecting terms of degree 2 and higher. This is what we wanted to prove.

Proof of Theorem 3.5.1. Computing the Lie algebra commutes with base change, so it is enough to consider the case $k=\mathbb{F}_{p}$.

By Proposition 3.5.2, the algebra $F_{n}$ is a quotient of a polynomial algebra. Therefore, to compute the Lie algebra $\operatorname{Lie}\left(\boldsymbol{\omega}_{n}\right)$, it is enough to know the coproduct of $F_{n}$ up to the first order. Indeed, a differentiation necessarily vanishes on monomials of higher degree.

Let $d_{w}: F_{n} \rightarrow \mathbb{F}_{p}$ be the differentiation which sends $X_{w}$ to 1 and $X_{v}$ to 0 for all $w \neq v \in X^{*}$. The bracket of two differentiations is given by $\left[d_{u}, d_{v}\right]=$ $\left(d_{u} \otimes d_{v}-d_{v} \otimes d_{u}\right) \circ \delta$, where $\delta$ is the co-product of $F_{n}$. It is easy to see that the bracket $\left[d_{u}, d_{v}\right]$ is trivial if $u$ and $v$ have the same length. Write $|u|=m$ and $|v|=n$, and suppose $m<n$. We compute

$$
\left[d_{u}, d_{v}\right]\left(X_{w}\right)= \begin{cases}v_{d} & \text { if } u \preceq \pi_{m}(w), \pi_{m}(w)-u=\pi_{m}(v), w_{m+1}+1=v_{m+1} \\ & \text { and }|w|=n, w_{m+2} \cdots w_{n}=v_{m+2} \cdots v_{n} \\ 0 & \text { otherwise. }\end{cases}
$$

From this we deduce

$$
\left[d_{u}, d_{v}\right]= \begin{cases}v_{m+1} \cdot d_{\left(\pi_{m}(v)+u\right)\left(v_{m+1}-1\right) v_{m+2} \cdots v_{n}} & \text { if } \pi_{m}(v)+u \preceq(p-1)^{m}, v_{m+1}>0, \\ 0 & \text { otherwise }\end{cases}
$$

In particular, for $u=(p-1)^{m}$, the bracket $\left[d_{u}, d_{v}\right]$ is non-zero if and only if $v=0^{m} x_{m+1} \cdots x_{n}$, with $x_{m+1}>0$. In this case we have

$$
\left[d_{(p-1)^{m}}, d_{0^{m} x_{m+1} \cdots x_{n}}\right]=x_{m+1} \cdot d_{(p-1)^{m}\left(x_{m+1}-1\right) x_{m+2} \cdots x_{n}} .
$$

Also, for $v=(p-1)^{n}$, the bracket $\left[d_{u}, d_{v}\right]$ is non-zero if and only if $u=0^{m}$. In this case we have

$$
\left[d_{0^{m}}, d_{(p-1)^{n}}\right]=-d_{(p-1)^{m}(p-2)(p-1)^{n-m-1}} .
$$


Consider the monoid homomorphism $a: X^{*} \rightarrow \mathbb{F}_{p}$ defined by $a(x)=\frac{(-1)^{x}}{x !}$ for $x \in X$. Set $\partial_{w}=a(w) d_{w}$ for all $w \in X^{*}$. In the basis $\partial_{w}$, the commutation relations (3.5.1) and (3.5.2) become

$$
\begin{aligned}
{\left[\partial_{0^{m}}, \partial_{(p-1)^{n}}\right] } & =-\partial_{(p-1)^{m}(p-2)(p-1)^{n-m-1}}, \\
{\left[\partial_{(p-1)^{m}}, \partial_{0^{m}} x_{n+1} \cdots x_{m}\right] } & =-\partial_{(p-1)^{m}\left(x_{m+1}-1\right) x_{m+2} \cdots x_{n}} .
\end{aligned}
$$

These are exactly the same relations as in [Bar05, Theorem 3.4], after replacing every letter $w_{i}$ of $w \in X^{*}$ by $p-1-w_{i}$. Thus, with these generators, the Lie graph of $\operatorname{Lie}\left(\boldsymbol{\omega}_{n}\right)$ coincides with that of $\operatorname{Lie}\left(\operatorname{Aut}_{p} X^{n}\right)$, pictured on [Bar05, Figure 1]. Therefore the two Lie algebras are isomorphic.

Remark 3.5.3. Incidentally, the lower central series of $\operatorname{Aut}_{p} X^{n}$ coincides with its dimension series (see Section 4.2). Thus the Lie algebra $\operatorname{Lie}\left(\operatorname{Aut}_{p} X^{n}\right)$ is restrictable. On the other hand, $\operatorname{Lie}\left(\boldsymbol{\omega}_{n}\right)$ is naturally a restricted Lie algebra. It would be interesting to know whether $\operatorname{Lie}\left(\operatorname{Aut}_{p} X^{n}\right)$ and $\operatorname{Lie}\left(\boldsymbol{\omega}_{n}\right)$ are isomorphic as restricted Lie algebras. 


\section{Chapter 4}

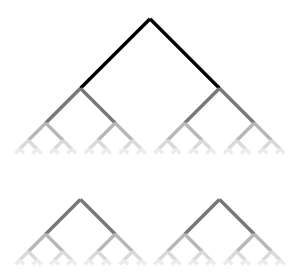

\section{Central Series and Automorphism Towers}

The lower central series of certain groups acting on rooted trees has been investigated by Kaloujnine [Kal95], Rozhkov [Roz96], Bartholdi and Grigorchuk [BG00, Bar05]. The automorphism tower of some of these groups has been tackled by Bartholdi and Sidki [BS06]. We present the dual results, describing the ideals associated to these sequences of groups. Quite amazingly, the lower central series and the automorphism tower of certain groups present similarities. This motivates us to treat both topics together in the present chapter.

\section{$4.1 \quad$ Notation}

We set $X=\mathbb{F}_{p}=k$, as in Chapter 2 .

\subsubsection{Two Splittings of $\mathbb{F}_{p}\{X\}$}

Recall the generating set $\left\{\Delta_{w}\right\}$ of the module $\mathbb{F}_{p}\{X\}$, as in Section 2.4.

Consider $d \geq 0$. Let $L_{d}$ be the low degree part of $\mathbb{F}_{p}\{X\}$ : the span of $\left\{\Delta_{w} \mid \operatorname{deg}(w)<d\right\}$. Let $H_{d}$ be the high degree part of $\mathbb{F}_{p}\{X\}$ : the span of $\left\{\Delta_{w} \mid \operatorname{deg}(w) \geq d\right\}$. It follows from these definitions that for $0 \leq d \leq p^{n}$, the intersection of $L_{d}$ with $\mathbb{F}_{p}\{X\}_{n}$ has dimension $d$. Similarly, the intersection of $H_{d}$ with $\mathbb{F}_{p}\{X\}_{n}$ has codimension $d$ in $\mathbb{F}_{p}\{X\}_{n}$. Also, we have the direct sum decomposition

$$
\mathbb{F}_{p}\{X\}=L_{d} \oplus H_{d}
$$

for all $d \geq 0$.

We define the height as the function

$$
\begin{aligned}
\mathrm{h}:\{0, \ldots, p-1\}^{*} & \rightarrow \mathbb{N} \\
x_{1} \ldots x_{n} & \mapsto \frac{p^{n}-1}{p-1}+\sum_{i=1}^{n}\left(p-1-x_{i}\right) p^{i-1} .
\end{aligned}
$$




\subsection{Notation}

It is not hard to see that $\mathrm{h}$ is bijective. Also, we have $\mathrm{h}(w)=\frac{p^{n}-1}{p-1}+\operatorname{deg}(w)$, where $n$ is the length of $w$. Furthermore, this function satisfies

$$
\mathrm{h}(v w)=\mathrm{h}(v)+p^{|v|} \mathrm{h}(w) .
$$

Consider $d \geq 0$. Let $T_{d}$ be the top part of $\mathbb{F}_{p}\{X\}$ : the span of $\left\{\Delta_{w} \mid \mathrm{h}(w)<\right.$ $d\}$. Let $B_{d}$ be the bottom part of $\mathbb{F}_{p}\{X\}$ : the span of $\left\{\Delta_{w} \mid \mathrm{h}(w) \geq d\right\}$. It follows from these definitions that $T_{d}$ has dimension $d$, and $B_{d}$ has codimension $d$ in $\mathbb{F}_{p}\{X\}$. Also, we have the direct sum decomposition

$$
\mathbb{F}_{p}\{X\}=T_{d} \oplus B_{d}
$$

for all $d \geq 0$.

\subsubsection{An Auxiliary Function}

Fix an integer $r \geq 1$. For each $0 \leq s<r$, we define the function

$$
\nu_{p}(r, s ; \bullet): \mathbb{N} \rightarrow \mathbb{N}
$$

as the only non-decreasing function satisfying

$$
\nu_{p}(r, s ; n)=n-(r-1) \frac{p^{k}-1}{p-1}-s p^{k},
$$

for all integers $k, n \geq 0$ so that

$$
r \frac{p^{k}-1}{p-1}+s p^{k} \leq n \leq r \frac{p^{k}-1}{p-1}+(s+1) p^{k}
$$

holds. It is readily checked that we have $\nu_{p}(1,0 ; n)=n$, and in general

$$
\sum_{s=0}^{r-1} \nu_{p}(r, s ; n)=n
$$

holds for all $r \geq 1$ and $n \geq 0$.

Some values of $\nu_{p}(r, s ; n)$ are given below:

\begin{tabular}{c|ccccccccccccc}
$n$ & 0 & 1 & 2 & 3 & 4 & 5 & 6 & 7 & 8 & 9 & 10 & 11 & $\cdots$ \\
\hline$\nu_{2}(2,0 ; n)$ & 0 & 1 & 1 & 2 & 3 & 3 & 3 & 4 & 5 & 6 & 7 & 7 & $\cdots$ \\
$\nu_{2}(2,1 ; n)$ & 0 & 0 & 1 & 1 & 1 & 2 & 3 & 3 & 3 & 3 & 3 & 4 & $\cdots$ \\
\hline$\nu_{3}(2,0 ; n)$ & 0 & 1 & 1 & 2 & 3 & 4 & 4 & 4 & 4 & 5 & 6 & 7 & $\cdots$
\end{tabular}

The function $\nu_{p}$ can be understood as follows. Consider $r$ copies of the infinite $p$-ary rooted tree. We mark the vertices of these trees step-by-step. At time 0, there is no marked vertex. Then at each time-step, we mark one vertex in a regular way, top to bottom, left to right. So at step 1, we mark the root of the first tree. At step 2 we mark the root of the second tree, and so on, until step $r$. At step $r+1$, we mark the first vertex of the first level of the first tree. We continue marking the first tree until time $r+p$, when the first level of the first tree is fully marked. Then we proceed to mark the first level of the second tree, and so on. At step $n$, the value $\nu_{p}(r, s ; n)$ is the number of marked vertices on the $(s+1)$-th tree. 


\subsection{Central Series}

The lower central series of a group $G$ is the sequence of subgroups $\gamma_{n}(G)$ defined by

$$
\begin{aligned}
\gamma_{1}(G) & =G, & \\
\gamma_{n}(G) & =\left[\gamma_{n-1}(G), G\right], & n>1 .
\end{aligned}
$$

The groups $\gamma_{n}(G)$ are easily seen to be characteristic in $G$. Each quotient $\gamma_{n}(G) / \gamma_{n+1}(G)$ is Abelian, and it is a standard fact that the $\mathbb{Z}$-module

$$
\mathfrak{L}(G)=\bigoplus_{n \geq 1} \gamma_{n}(G) / \gamma_{n+1}(G)
$$

is a graded Lie ring over $\mathbb{Z}$, where the Lie bracket of homogeneous elements is induced by commutation in $G$.

Fix a prime number $p$. The dimension series of a group $G$ is the sequence of subgroups $\gamma_{n}^{(p)}(G)$ defined by

$$
\begin{aligned}
& \gamma_{1}^{(p)}(G)=G, \\
& \gamma_{n}^{(p)}(G)=\left[\gamma_{n-1}^{(p)}(G), G\right] \mho_{p}\left(G_{\lceil n / p\rceil}\right), \quad n>1,
\end{aligned}
$$

where $\mho_{p}(H)$ is the group generated by $p$-th powers in $H$. This series also goes with the names of Brauer, Jennings, Lazard, Zassenhaus, ...

The groups $\gamma_{n}^{(p)}(G)$ are easily seen to be characteristic in $G$. Each quotient $\gamma_{n}^{(p)}(G) / \gamma_{n+1}^{(p)}(G)$ is elementary Abelian, and it is a standard fact that the $\mathbb{F}_{p^{-}}$ vector space

$$
\mathfrak{L}_{p}(G)=\bigoplus_{n \geq 1} \gamma_{n}^{(p)}(G) / \gamma_{n+1}^{(p)}(G)
$$

is a graded, restricted Lie algebra over $\mathbb{F}_{p}$ (see [Jac79, Section V.7] for the definition of restricted Lie algebras). On homogeneous elements, the Lie bracket is induced by commutation, and the $p$-operation is induced by raising to the $p$-th power in $G$.

\subsection{1 $\operatorname{Aut}_{p} X^{*}$}

Let $\gamma_{n}$ be the $n$-th term of the lower central series of Aut $_{p} X^{*}$. The groups $\gamma_{n}$ (or rather the finite quotients $\left.\pi_{m}\left(\gamma_{n}\right)\right)$ have been described by Kaloujnine in [Kal95] and the associated graded Lie algebra structure has been investigated in [SN05] and in [Bar05]. We partly rephrase these results using our notation.

Theorem 4.2.1 (cf. [Kal95, Théorème 6] and [Bar05, Theorem 3.4]). The lower central and the dimension series of $\operatorname{Aut}_{p} X^{*}$ coincide, and the ideal $I_{n}=\mathcal{I}_{p}\left(\gamma_{n+1}\right)$ is generated by $\left[L_{n}\right]$. 


\subsection{Central Series}

Proof. From Figure 1 of [Bar05], it is easy to deduce a nice generating set for $\mathcal{I}\left(\gamma_{n+1}\right)$. Consider the first $n$ columns of the graph, and then read bottom to top, left to right. Using the rule $w \mapsto\left[\Delta_{\bar{w}}\right]$, we get the desired generating set. Here we write $\bar{w}$ for the word $w$ where each letter $x$ is replaced by $p-1-x$.

This holds because $w\left(x_{0}\right)$ is in a sense dual to $\left[\Delta_{\bar{w}}\right]$ : by definition we have $\left[\Delta_{w}\right]\left(w^{\prime}\left(x_{0}\right)\right) \neq 0$ if and only if $w^{\prime}=\bar{w}$.

\subsubsection{The Grigorchuk Group}

Let $\mathrm{G}$ be the Grigorchuk group (see Section A.1). The lower central series of $\mathrm{G}$ has been computed by Rozhkov [Roz96], and by Bartholdi and Grigorchuk [BG00] (see also [Bar05]).

Theorem 4.2.2 (cf. [BG00, Theorem 6.4]). Let $\gamma_{n}$ be the $n$-th term of the lower central series of $\mathrm{G}$. Then $\mathcal{I}\left(\gamma_{n+1}\right)$ is generated, modulo $\mathcal{I}\left(\gamma_{n}\right)$ :

- for $n=1$, by $\left\{[\varnothing],[\Delta],\left[\Delta^{2}\right]\right\}$;

- for $n=2$, by $\left\{\left[\Delta_{0}\right],\left[\Delta_{01}\right]\right\}$;

- for $n \geq 3$, by $T_{n-1} *\left[\Delta_{0}\right]$ and $T_{\nu_{2}(2,0 ; n-2)} *\left[\Delta_{00}+\Delta_{001}\right]$.

Proof. This is a direct consequence of the original theorem, except for the notation. For convenience, note that the module $V_{n}^{d}$ and the group $N_{m}$ from [BG00] correspond to our $M_{p^{n}-d}^{(n)}$ and $X^{m-1} *\left(K^{2} \cdot(X * K)\right)$ respectively.

The group $K / N_{1}$ is isomorphic to $C_{2}$, a cyclic group of order 2. Dually we have

$$
\mathcal{I}\left(N_{1}\right)=\mathcal{I}(K)+\left(\left[\Delta_{0}\right]\right) .
$$

Next, $N_{1} / N_{2}$ is isomorphic to $C_{2}^{3}$, and the functions $\left[\Delta_{10}\right]$, $\left[\Delta_{00}\right]$ and $\left[\Delta_{001}\right]$ form a base of the dual of $N_{1} / N_{2}$. A straightforward computation shows that the annihilator of $\left[\Delta_{00}+\Delta_{001}\right]$ in this group is $(X * K) / N_{2}$, as needed.

The dimension series of $\mathrm{G}$ is also computed in [BG00] and in [Bar05].

Theorem 4.2.3 (cf. [BG00, Theorem 6.6]). Let $\gamma_{n}^{(p)}$ be the $n$-th term of the dimension series of $\mathrm{G}$. Then $\mathcal{I}\left(\gamma_{n+1}^{(p)}\right)$ is generated, modulo $\mathcal{I}\left(\gamma_{n}^{(p)}\right)$ :

- for $n=1$, by $\left\{[\varnothing],[\Delta],\left[\Delta^{2}\right]\right\}$;

- for $n=2$, by $\left\{\left[\Delta_{0}\right],\left[\Delta_{01}\right]\right\}$;

- for $n \geq 3$, by $T_{n-1} *\left[\Delta_{0}\right]$ and $T_{\left\lfloor\frac{n-2}{2}\right\rfloor} *\left[\Delta_{00}+\Delta_{001}\right]$.

Proof. This is again just a change of notation from the original theorem. 


\subsubsection{The Gupta-Sidki Group}

Consider $p=3$ and let $\ddot{\Gamma}$ be the Gupta-Sidki group (see Section A.3). The lower central series of $\ddot{\Gamma}$ has been computed in [Bar05].

We have $[\Delta](\ddot{\Gamma})=0$, allowing us to deduce, via Proposition 2.4.2, that the action of $\ddot{\Gamma}$ on $\mathbb{F}_{3}\{X\}_{n}$ is not uniserial for $n \geq 2$. Consequently, the lower central series of $\ddot{\Gamma}$ descends quicker than for more "tame" examples, and the rank of $\gamma_{n}(\ddot{\Gamma}) / \gamma_{n+1}(\ddot{\Gamma})$ is unbounded.

Let $\alpha_{n}$ be the sequence of integers defined by

$$
\alpha_{1}=1, \quad \alpha_{2}=2, \quad \alpha_{n}=2 \alpha_{n-1}+\alpha_{n-2} \quad \text { for } n \geq 3 .
$$

Asymptotically, $\alpha_{n}$ grows like $(1+\sqrt{2})^{n}$, slower than $3^{n}$. For $r=1,2$, we define the function

$$
\begin{aligned}
\widetilde{h}_{r}:\{0, \ldots, p-1\}^{*} & \rightarrow \mathbb{N}, \\
x_{1} \ldots x_{n} & \mapsto r \alpha_{n+1}+\sum_{i=1}^{n}\left(p-1-x_{i}\right) \alpha_{i} .
\end{aligned}
$$

For $d \geq 0$, we let $\widetilde{T}_{d}^{(r)}$ be the span of $\left\{\Delta_{w} \mid \widetilde{h}_{r}(w)<d\right\}$.

Theorem 4.2.4 (cf. [Bar05, Theorem 3.8]). The lower central and the dimension series of $\ddot{\Gamma}$ coincide. Writing $\gamma_{n}$ for the $n$-th term of the lower central series of $\ddot{\Gamma}$, the ideal $\mathcal{I}_{3}\left(\gamma_{n+1}\right)$ is generated, modulo $\mathcal{I}_{3}\left(\gamma_{n}\right)$ :

- for $n=1$, by $\left\{[\varnothing],\left[\Delta_{1}\right]\right\}$;

- for $\geq 2$, by $\widetilde{T}_{n}^{(1)} *\left[\Delta_{0}\right]$ and $\widetilde{T}_{n}^{(2)} *\left[\Delta_{01}\right]$.

Proof. This is a direct consequence of the original theorem, except for the notation. Set $c=[a, t]$ and $u=[a, c]$. Consider the branching subgroup $K=[\ddot{\Gamma}, \ddot{\Gamma}]$ of $\ddot{\Gamma}$. Since it contains $\operatorname{Stab}_{\ddot{\Gamma}}(2)$, it is enough to compute modulo $\operatorname{Stab}(3)$ to see all what is happening in $K /(X * K)$. This amounts to looking at functions in $\mathcal{P}_{3}$.

Write $K_{1}=\pi_{3}(K)$ and $K_{n+1}=\left[K_{n}, \pi_{3}(\Gamma)\right]$. It is easy to check that $K_{5}$ is the trivial group. Also, $K_{1} / K_{2}$ is isomorphic to $C_{3}$, a cyclic group of order 3 , generated by $c$. Dually, we have

$$
\mathcal{I}_{3}\left(K_{2}\right)=\mathcal{I}_{3}\left(K_{1}\right)+\left(\left[\Delta_{0}\right]\right) .
$$

Next, $K_{2} / K_{3}$ is isomorphic to $C_{3}^{2}$. The elements $\left[\Delta_{01}\right]$ and $\left[\Delta_{20}\right]$ form a basis of the dual of this group. The corresponding dual basis is given by the image of $u^{-1}$ and $0 * c$. Finally, $K_{n} / K_{n+1}$ is isomorphic to $C_{3}$ for $n=3,4$, and the corresponding functions are $\left[\Delta_{10}\right]$ and $\left[\Delta_{00}\right]$.

\subsubsection{The Fabrykowski-Gupta Group}

Consider $p=3$ and let $\Gamma$ be the Fabrykowski-Gupta group (see Section A.4). The lower central series of $\Gamma$ has been computed in [Bar05]. 


\subsection{Automorphism and Normalizer Towers}

Theorem 4.2.5 (cf. [Bar05, Theorem 3.13]). Let $\gamma_{n}$ be the $n$-th term of the lower central series of $\Gamma$. Then $\mathcal{I}_{3}\left(\gamma_{n+1}\right)$ is generated, modulo $\mathcal{I}_{3}\left(\gamma_{n}\right)$ :

- for $n=1$, by $\left\{[\varnothing],\left[\Delta_{2}\right]\right\}$;

- for $n=2$, by $\left[\Delta_{1}\right]$;

- for $n=3$, by $\left[T_{8}\right]$,

- for $n \geq 4$, by

$$
\begin{array}{rlr}
T_{\nu_{3}(5,0 ; n-3)} *\left[\Delta_{11}\right], & T_{\nu_{3}(5,1 ; n-3)} *\left[\Delta_{01}\right], \\
T_{\nu_{3}(5,2 ; n-3)} *\left[\Delta_{20}\right], & T_{\nu_{3}(5,3 ; n-3)} *\left[\Delta_{10}\right], \\
T_{\nu_{3}(5,4 ; n-3)} *\left[\Delta_{00}\right], & \\
\text { and } T_{\nu_{3}(2,0 ; n-2)} *\left[-\Delta_{0}+\Delta_{21}\right] . &
\end{array}
$$

Proof. This is a direct consequence of the original theorem, except for the notation. Set $c=[a, r]$ and $u=[a, c]$. Consider the branching subgroup $K=[\Gamma, \Gamma]$ of $\Gamma$. Since it contains $\operatorname{Stab}_{\Gamma}(2)$, it is enough to compute modulo $\operatorname{Stab}(3)$ to see all what is happening in $K /(X * K)$. This amounts to looking at functions in $\mathcal{P}_{3}$.

Write $K_{1}=\pi_{3}(K)$ and $K_{n+1}=\left[K_{n}, \pi_{3}(\Gamma)\right]$. It is easy to check that $K_{8}$ is the trivial group. Also, $K_{1} / K_{2}$ is isomorphic to $C_{3}$, a cyclic group of order 3 . Dually, we have

$$
\mathcal{I}_{3}\left(K_{2}\right)=\mathcal{I}_{3}\left(K_{1}\right)+\left(\left[\Delta_{1}\right]\right) .
$$

Next, $K_{2} / K_{3}$ is isomorphic to $C_{3}^{2}$. The elements $\left[\Delta_{0}\right]$ and $\left[\Delta_{21}\right]$ form a basis of the dual of this group, and the annihilator of $\left[-\Delta_{0}+\Delta_{21}\right]$ has rank 1 , generated by $(0 * c) / u$. Finally, $K_{n} / K_{n+1}$ is isomorphic to $C_{3}$ for $n=3,4,5,6,7$, and the corresponding functions are $\left[\Delta_{11}\right],\left[\Delta_{01}\right],\left[\Delta_{20}\right],\left[\Delta_{10}\right],\left[\Delta_{00}\right]$.

\subsection{Automorphism and Normalizer Towers}

Lavreniuk and Nekrashevych [LN02] proved that any automorphism of a saturated, weakly branch group is induced by conjugation in Aut $X^{*}$. Thus the automorphism group of such a group coincides with its normalizer in Aut $X^{*}$.

The automorphism tower of a group $G$ is the sequence of groups defined inductively by

$$
\operatorname{Aut}^{0} G=G, \quad \operatorname{Aut}^{n+1} G=\operatorname{Aut}\left(\operatorname{Aut}^{n} G\right) .
$$

If $G$ is a subgroup of $\operatorname{Aut}_{p} X^{*}$, we define its normalizer tower as

$$
\operatorname{Norm}^{0} G=G, \quad \operatorname{Norm}^{n+1} G=\operatorname{Norm}\left(\operatorname{Norm}^{n} G\right),
$$

where Norm designates the normalizer in $\operatorname{Aut}_{p} X^{*}$. 


\subsubsection{The Grigorchuk Group}

Let $\mathrm{G}$ be the Grigorchuk group (see Section A.1). Then we know that $\mathcal{I}(G)$ is generated, as a branching ideal, by

$$
\begin{aligned}
P_{\Delta} & =\left[\Delta+\Delta^{2}+\Delta^{3}\right], & P_{0}=\left[\underline{0}+\underline{1} *\left(\Delta+\Delta^{2}\right)\right], \\
Q & =[\underline{0}] \cdot[\underline{1}]+[\Delta * \underline{1}+\Delta * \underline{0} * \Delta] . &
\end{aligned}
$$

The group $\mathrm{G}$ is saturated and weakly branch, therefore its automorphism tower coincides with its normalizer tower.

Theorem 4.3.1 (cf. [BS06, Theorem 6.1]). Let Aut ${ }^{n} \mathrm{G}$ be the $n$-th term in the automorphism tower of $\mathrm{G}$. Then $\mathcal{I}\left(\right.$ Aut $\left.^{n} \mathrm{G}\right)$ is equal to $\left(B_{\nu_{2}(2,0 ; n-2)} * P_{\Delta}\right)+\left(B_{n-1} *\right.$ $\left.P_{0}\right)+\left(H_{n} * Q\right)$.

Proof. Write

$$
p=1 *(\text { adad }), \quad q=1 *(a d), \quad r=1 * a .
$$

Then it is straightforward to check that we have

$$
\begin{array}{lll}
P_{\Delta}(p)=0, & P_{0}(p)=0, & Q(p)=1, \\
P_{\Delta}(q)=0, & P_{0}(q)=1, & \\
P_{\Delta}(r)=1 . & &
\end{array}
$$

A comparison with the original theorem shows that the polynomials vanish in the given order.

\subsubsection{The Gupta-Sidki Group}

Let $\ddot{\Gamma}$ be the Gupta-Sidki group (see Section A.3). The automorphism tower of $\ddot{\Gamma}$ is described in [BS06]. It coincides with the normalizer tower of the group in Aut $X^{*}$, and stops after two steps. On the other hand, the normalizer tower inside $\mathrm{Aut}_{3} X^{*}$ grows more slowly. The following theorem concerns the latter tower.

Theorem 4.3.2 (cf. [BS06, Theorem 5.13]). Let $\operatorname{Norm}^{n} \ddot{\Gamma}$ be the $n$-th term in the normalizer tower of $\ddot{\Gamma}$ in Aut $_{3} X^{*}$. Then $\mathcal{I}_{3}\left(\operatorname{Norm}^{n} \ddot{\Gamma}\right)$ is contained in $\mathcal{I}_{3}(\ddot{\Gamma}) \cap$ $\left(\left[H_{n}\right]\right)$.

Proof. Since $\operatorname{Norm}^{n} \ddot{\Gamma}$ contains $\ddot{\Gamma}$, we have $\mathcal{I}_{3}\left(\operatorname{Norm}^{n} \ddot{\Gamma}\right) \subseteq \mathcal{I}_{3}(\ddot{\Gamma})$. The elements of the original theorem exactly generate the annihilator of $\left[H_{n}\right]$, whence the claim.

This theorem is not a full description of $\operatorname{Norm}^{n} \ddot{\Gamma}$. On the other hand, note that $\ddot{\Gamma}$ is probably a little more tricky than other groups, because its action on $\mathbb{F}_{3}\{X\}_{n}$ is not uniserial for $n \geq 2$. For example, this allows its lower central series to descend quicker than for $\Gamma$. 



\section{Chapter 5}

\section{Hausdorff Dimension}

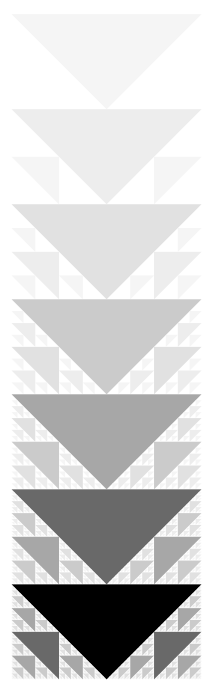

Hausdorff dimension of profinite groups has first been approached by Abercrombie [Abe94]. In [Sie08], I have computed the Hausdorff dimension of certain subgroups of Aut $X^{*}$. This article is reprinted in Appendix B. It contains the first explicit examples of finitely generated groups with irrational dimension, as well as the first example of a finitely generated group of dimension 1. Moreover, all these groups act transitively on each level of the tree. Although it has been proved by Abért and Virág [AV05] that any $\alpha \in[0,1]$ can be realized as the Hausdorff dimension of a finitely generated group, it is not known whether this still holds for finitely generated, level-transitive groups.

We introduce Hausdorff dimension in Section 5.1. Section 5.2 is dedicated to layers, a concept tightly connected to dimension. In Section 5.3 we give formulæ to compute the Hausdorff dimension from its modules of layers, and from a nice generating set. We thus relate to the rest of this work. Section 5.4 is independent from the others. It is devoted to a group which has dimension 1 and is branch.

\section{$5.1 \quad$ Definition}

We focus on subgroups of $\operatorname{Aut}_{p} X^{*}$, although Hausdorff dimension can equally well be defined for subgroups of Aut $X^{*}$. On the other hand, layers are particularly well suited for $\operatorname{Aut}_{p} X^{*}$.

Let $G$ be a closed subgroup of $\operatorname{Aut}_{p} X^{*}$. We can endow $G$ with a metric by setting

$$
d(g, h)=\frac{1}{\mid \operatorname{Aut}_{p} X^{n \mid}},
$$

where $n$ is maximal so that $\pi_{n}(g)=\pi_{n}(h)$. The Hausdorff dimension of $G$ is given by the formula [Abe94,BS97]:

$$
\operatorname{dim}_{H} G=(p-1) \liminf _{n \rightarrow \infty} \frac{\log _{p}\left|\pi_{n}(G)\right|}{p^{n}} .
$$

This yields the Hausdorff dimension of $G$ as a metric space. The normalisation of the metric is chosen so that $\operatorname{dim}_{H} \operatorname{Aut}_{p} X^{*}=1$. It has been shown in [Abe94] 


\subsection{Layers}

that the Hausdorff dimension always coincides with the lower box dimension for profinite groups, and the above equality is easily seen to yield the lower box dimension of $G$.

\section{$5.2 \quad$ Layers}

Let $G$ be a closed subgroup of $\operatorname{Aut}_{p} X^{*}$. The $n$-th layer of $G$ is

$$
L_{n}(G)=\pi_{n+1}\left(\operatorname{Stab}_{G}(n)\right) .
$$

This is an elementary Abelian $p$-group, and we view it as a submodule of $\mathbb{F}_{p}\{X\}_{n}^{\circ}$ (see Section 1.6). The action of $g \in G$ is via conjugation by $g^{-1}$. The $n$-th co-layer of $G$ is

$$
C_{n}(G)=L_{n}(G)^{\perp}
$$

the orthogonal of $L_{n}(G)$. This is a submodule of $\mathbb{F}_{p}\{X\}_{n}$. The action of $G$ on $C_{n}(G)$ is the one induced by the action of $G$ on $X^{n}$, as in Equation (1.6.1). We note the relation

$$
\operatorname{dim}_{\mathbb{F}_{p}}\left(L_{n}(G)\right)+\operatorname{dim}_{\mathbb{F}_{p}}\left(C_{n}(G)\right)=p^{n} .
$$

We form the product $L_{*}(G)=\prod_{n>0} L_{n}(G) \subseteq \mathbb{F}_{p}\{\{X\}\}$ and the sum $C_{*}(G)=$ $\bigoplus_{n \geq 0} C_{n}(G) \subseteq \mathbb{F}_{p}\{X\}$. We call these the module of layers and the module of colayers of $G$, respectively. It is immediate that $L_{*}(G)$ is the orthogonal of $C_{*}(G)$, and both are $G$-modules.

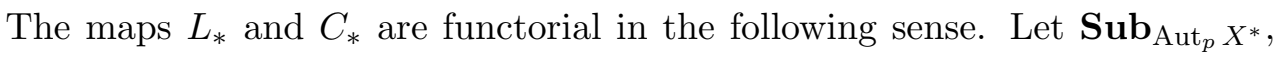
$\operatorname{Sub}_{\mathbb{F}_{p}\{\{X\}\}}$ and $\mathbf{S u b}_{\mathbb{F}_{p}\{X\}}$ be the category of closed subgroups of $\operatorname{Aut}_{p} X^{*}$, of closed subspaces of $\mathbb{F}_{p}\{\{X\}\}$, and of subspaces of $\mathbb{F}_{p}\{X\}$ respectively. In each case, the morphisms we consider are all the inclusions. Then

$$
L_{*}: \mathbf{S u b}_{\mathrm{Aut}_{p} X^{*}} \rightarrow \mathbf{S u b}_{\mathbb{F}_{p}\{\{X\}\}}, \quad C_{*}: \mathbf{S u b}_{\mathrm{Aut}_{p} X^{*}} \rightarrow \mathbf{S u b}_{\mathbb{F}_{p}\{X\}}
$$

are functors. $L_{*}$ is covariant, and $C_{*}$ is contravariant.

Proposition 5.2.1. Let $G$ be a closed subgroup of $\operatorname{Aut}_{p} X^{*}$. The following implications hold:

(i) $G$ is self-similar $\Longrightarrow L_{*}(G)$ is self-similar $\Longleftrightarrow C_{*}(G)$ is branching;

(ii) $G$ is branching $\Longrightarrow L_{*}(G)$ is branching $\Longleftrightarrow C_{*}(G)$ is self-similar.

Proof. The equivalences

$$
\begin{aligned}
L_{*}(G) \text { is self-similar } & \Longleftrightarrow C_{*}(G) \text { is branching, } \\
L_{*}(G) \text { is branching } & \Longleftrightarrow C_{*}(G) \text { is self-similar }
\end{aligned}
$$

are immediate from Equation 1.6.2. 
For the other implications, consider an integer $m \geq 0$ and $v \in X^{*}$ of length $n \leq m$. If $G$ is self-similar, then $\operatorname{Stab}_{G}(m) @ v$ is a subset of $\operatorname{Stab}_{G}(m-n)$. This implies $L_{m}(G) @ v \subseteq L_{m-n}(G)$, and so $L_{*}(G)$ is self-similar.

If $G$ is branching we consider $m \geq 0$ and $v \in X^{*}$ of length $n \geq 0$. Then $v * \operatorname{Stab}_{G}(n) \leq \operatorname{Stab}_{G}(m+n)$ implies $v * L_{m}(G) \subseteq L_{m+n}(G)$, and thus $L_{*}(G)$ is branching.

Example 5.2.2. We investigate the Grigorchuk group a little further (see Section A.1 for the definition). For $n \geq 3$, the $n$-th co-layer of the group $\mathrm{G}$ is generated by $v * \Delta_{111}, v * \Delta_{011}$ and $v * \Delta_{101}$ with $v$ ranging over $X^{n-3}$. The module $C_{*}(\mathrm{G})$ is clearly branching.

The above example shows a behaviour which turns out to be common to all subgroups of $\operatorname{Aut}_{p} X^{*}$. This is expressed in the following proposition. For reference, nice generating sets are defined in Section 2.3. They always exist by Proposition 2.3.3.

Proposition 5.2.3. Let $G$ be a closed subgroup of $\operatorname{Aut}_{p} X^{*}$, and let $S$ be a nice generating set for $\mathcal{I}_{p}(G)$. Then $C_{*}(G)$ is generated by the preimages of $\operatorname{lin}(S)$ under $[\bullet]: \mathbb{F}_{p}\{X\} \rightarrow \mathcal{P}_{*}$.

Proof. Let $S_{n} \subseteq S$ be the set of functions of depth $n$. Then $\operatorname{lin}\left(S_{n}\right)$ generates the orthogonal of $\pi_{n}\left(\operatorname{Stab}_{G}(n-1)\right)$; this is precisely $C_{n-1}(G)$.

Corollary 5.2.4. Let $G$ be a closed subgroup of $\operatorname{Aut}_{p} X^{*}$, let $S$ be a nice generating set for $\mathcal{I}_{p}(G)$, and let $d_{n}$ be the number of functions of depth $n$ in $S$. Then $d_{n+1}=\operatorname{dim}_{\mathbb{F}_{p}}\left(C_{n}(G)\right)$ for all $n \geq 0$.

Proof. By definition of a nice generating set, $\operatorname{lin}(S)$ is a linearly independent set.

Let $G \leq H$ be closed subgroups of $\operatorname{Aut}_{p} X^{*}$. We have the inclusions $L_{*}(G) \leq$ $L_{*}(H)$ and $C_{*}(G) \geq C_{*}(H)$. Viewing these as Abelian groups, the indices $\left[L_{*}(H): L_{*}(G)\right]$ and $\left[C_{*}(G): C_{*}(H)\right]$ make sense, and are equal to $p^{n}$, where $n$ is the dimension of the quotient vector space. We have

Lemma 5.2.5. For all closed subgroups $G \leq H$ of $\operatorname{Aut}_{p} X^{*}$, the equalities $[H$ : $G]=\left[L_{*}(H): L_{*}(G)\right]=\left[C_{*}(G): C_{*}(H)\right]$ hold.

Proof. The projection of $L_{*}(G)$ onto $\prod_{i=0}^{n-1} \mathbb{F}_{p}\{X\}_{i}$ has the same number of elements as $\pi_{n}(G)$, and similarly for $H$. Thus the first equality is obvious. The second equality follows by duality, from Equation (5.2.1).

Another way to state this lemma is the relation

$$
\log _{p}[H: G]=\operatorname{dim}_{\mathbb{F}_{p}}\left(L_{*}(H) / L_{*}(G)\right)=\operatorname{dim}_{\mathbb{F}_{p}}\left(C_{*}(G) / C_{*}(H)\right) .
$$

Example 5.2.6. We come back to Example 5.2.2. The module generated by $C_{*}(\mathrm{G})$ and $\varnothing, \Delta^{1}, \Delta^{2}$ is the module of co-layers of $\mathrm{G}^{\prime}$, the derived subgroup of $\mathrm{G}$. We have $\left[\mathrm{G}: \mathrm{G}^{\prime}\right]=2^{3}$.

Also, the self-similar closure of $C_{*}(\mathrm{G})$ is $C_{*}(K)$, generated by $C_{*}(\mathrm{G})$ together with $\varnothing, \Delta^{1}, \Delta^{2}$ and $\Delta_{01}$. Here $C_{*}(K)$ is the module of co-layers of $K=\left(X^{*}\right)^{-1} *$ $G$, the branching core of $G$. We have $[G: K]=2^{4}$. 


\subsection{Computing Dimensions}

\subsection{Computing Dimensions}

We define a metric on $L_{*}(G)$ by

$$
d(g, h)=\frac{1}{\left|\mathbb{F}_{p}\{X\}_{<n}^{\circ}\right|},
$$

where $n$ is maximal so that $\pi_{n}(g)=\pi_{n}(h)$. In a similar way we define a metric on $C_{*}(G)$. Write

$$
l_{n}=\operatorname{dim}_{\mathbb{F}_{p}}\left(\pi_{n}\left(L_{*}(G)\right)\right), \quad c_{n}=\operatorname{dim}_{\mathbb{F}_{p}}\left(\pi_{n}\left(C_{*}(G)\right)\right) .
$$

Proposition 5.3.1. The upper and lower box dimension of $L_{*}(G)$ and of $C_{*}(G)$ are given by the formula:

$$
\begin{aligned}
& \overline{\operatorname{dim}}_{\square} L_{*}(G)=(p-1) \limsup _{n \rightarrow \infty} \frac{l_{n}}{p^{n}}, \quad \underline{\operatorname{dim}}_{\square} L_{*}(G)=(p-1) \liminf _{n \rightarrow \infty} \frac{l_{n}}{p^{n}}, \\
& \overline{\operatorname{dim}}_{\square} C_{*}(G)=(p-1) \limsup _{n \rightarrow \infty} \frac{c_{n}}{p^{n}}, \quad \underline{\operatorname{dim}_{\square}} C_{*}(G)=(p-1) \liminf _{n \rightarrow \infty} \frac{c_{n}}{p^{n}} .
\end{aligned}
$$

Proof. We prove the formulæ for $L_{*}(G)$; the proof for $C_{*}(G)$ is the same.

The distance on $L_{*}(G)$ is ultrametric. We cover $L_{*}(G)$ with cosets of $\pi_{n}^{-1}(1)$. These have diameter $1 /\left|\mathbb{F}_{p}\{X\}_{<n}^{\circ}\right|$, and we need $l_{n}$ of them. Since this covering is disjoint, and the distance takes only the values $1 /\left|\mathbb{F}_{p}\{X\}_{<n}^{\circ}\right|$, we deduce that this is an optimal covering for this diameter. The formulæ are found by replacing $\log _{p}\left|\mathbb{F}_{p}\{X\}_{<n}^{\circ}\right|=\frac{p^{n}-1}{p-1}$.

Corollary 5.3.2. For any closed subgroup $G \leq \operatorname{Aut}_{p} X^{*}$, the equalities

$$
\overline{\operatorname{dim}}_{\square} L_{*}(G)+\underline{\operatorname{dim}}_{\square} C_{*}(G)=\underline{\operatorname{dim}}_{\square} L_{*}(G)+\overline{\operatorname{dim}}_{\square} C_{*}(G)=1
$$

hold.

Proof. Equation (5.2.1) implies

$$
l_{n}+c_{n}=\frac{p^{n}-1}{p-1}
$$

for all $n \geq 0$. The claim follows.

Corollary 5.3.3. Let $G$ be a closed subgroup of $\operatorname{Aut}_{p} X^{*}$. We have the equalities

$$
\operatorname{dim}_{H} G=\underline{\operatorname{dim}}_{\square} L_{*}(G)=1-\overline{\operatorname{dim}}_{\square} C_{*}(G) .
$$

Proof. This is a consequence of Equation (5.1.1) and of Corollary 5.3.2, using the equality $l_{n}=\log _{p}\left|\pi_{n}(G)\right|$.

Together with Proposition 5.2.3, this shows that the Hausdorff dimension of a group $G$ can be read off a nice generating set of $\mathcal{I}_{p}(G)$. This is even more true for self-similar groups. 
Let $G$ be a closed self-similar subgroup of $\operatorname{Aut}_{p} X^{*}$. Consider the sequence $r_{n}$ defined by

$$
r_{0}=\operatorname{dim}_{\mathbb{F}_{p}} C_{0}(G), \quad r_{n}=\operatorname{dim}_{\mathbb{F}_{p}}\left(C_{n}(G) /\left\langle X * C_{n-1}(G)\right\rangle\right),
$$

for $n>0$. Since $G$ is self-similar, $C_{n}(G)$ contains $X * C_{n-1}(G)$ by Lemma 5.2.1, and so the definition makes sense.

The following proposition shows that $r_{n}$ measures the number of nice functions of depth $n+1$ which are needed in a nice generating set of $\mathcal{I}_{p}(G)$, removing the functions we obtain using the fact that $\mathcal{I}_{p}(G)$ is branching.

Proposition 5.3.4. Let $G$ be a closed subgroup of $\operatorname{Aut}_{p} X^{*}$, let $S$ be a nice generating set for $\mathcal{I}_{p}(G)$, and let $d_{n}$ be the number of functions of depth $n$ in $S$. Then $r_{n}=d_{n+1}-p d_{n}$ holds for all $n \geq 0$.

Proof. This follows directly from Corollary 5.2.4.

Theorem 5.3.5. Let $G$ be a closed self-similar subgroup of $\operatorname{Aut}_{p} X^{*}$. The equality

$$
\operatorname{dim}_{H} G=1-\sum_{n \geq 0} \frac{r_{n}}{p^{n}}
$$

holds.

Proof. Using Propositions 5.2.3 and 5.3.4, we have the relation

$$
c_{n}=\sum_{i=0}^{n-1} \frac{p^{n-i}-1}{p-1} r_{i} .
$$

We obtain the result by replacing this in the formula for $\overline{\operatorname{dim}}_{\square} C_{*}(G)$. Since the sequence $\frac{r_{n}}{p^{n}}$ is non-negative, we can discard the lim sup.

This theorem is extremely efficient for regular branch groups, because in this case the sum is finite, due to Theorem 2.2.7. Here are two examples.

Example 5.3.6. Consider the Grigorchuk group G. We know that $\mathcal{I}(\mathrm{G})$ is generated by three functions of depth 4 , as a branching ideal. Therefore $r_{3}=3$ and $r_{n}=0$ for $n \neq 3$. This yields $\operatorname{dim}_{H} \mathrm{G}=1-\frac{3}{2^{3}}=\frac{5}{8}$.

Example 5.3.7. Consider the Gupta-Sidki group $\ddot{\Gamma}$. We know that, as a branching ideal, $\mathcal{I}_{3}(\ddot{\Gamma})$ is generated by one function of depth 2 and two functions of depth 3. Therefore $r_{1}=1, r_{2}=2$, and $r_{n}=0$ for $n \neq 1,2$. This yields $\operatorname{dim}_{H} \ddot{\Gamma}=1-\frac{1}{3}-\frac{2}{3^{2}}=\frac{4}{9}$. 


\subsection{Dimension 1}

\subsection{Dimension 1}

In [Sie08] I constructed an example of a 2-generated group acting on the binary tree, with Hausdorff dimension 1. In an attempt to answer a question of Grigorchuk, here is a slight variation of this example. This new group has dimension 1, and is easily seen to be branch, i.e. $\operatorname{Rist}_{G}(n)$ has finite index in $G$ for all $n$. The method is the same as in [Sie08, Section 6], where more details are given.

We first need the group $G_{n}=\left\langle a, b_{(1, n)}, \ldots, b_{(n, n)}\right\rangle$ where the generators are defined recursively by

$$
\begin{aligned}
a & =\sigma, \\
b_{(i, n)} & =\left(1, b_{(i+1, n)}\right) \\
b_{(n, n)} & =\left(a, b_{(1, n)}\right) .
\end{aligned} \quad \text { for } i=1, \ldots, n-1,
$$

We shall need three facts about the group $G_{n}$ :

- $G_{n}$ is self-similar;

- each generator has order 2 , and thus $G_{n} / G_{n}^{\prime}$ is finite;

- $\operatorname{dim}_{H} G_{n}=1-\frac{3}{2^{n+1}}$.

The first two facts are easy to check, and the third one is proved in [Sie08] and in [Šun07].

Given $t$ automorphisms $g_{1}, \ldots, g_{t}$ of $X^{*}$, we define a new one by

$$
\delta\left(g_{1}, \ldots, g_{t}\right)=\prod_{i=0}^{t-1}\left(\underline{1}^{i} \underline{0}^{t-i}\right) * g_{i+1} .
$$

Note that $\delta\left(g_{1}, \ldots, g_{t}\right)$ is in $\operatorname{Stab}(t)$. We shall also need the element $d=(a, d)$, and we set

$$
c_{n}=\delta\left(a, d, c_{n+1}, b_{(1,1)}, b_{(1,2)}, b_{(2,2)}, \ldots, b_{(1, n)}, \ldots, b_{(n, n)}\right),
$$

with $t_{n}=3+\frac{n(n+1)}{2}$.

We consider the groups $H_{n}=\left\langle a, d, c_{n}\right\rangle$ and $L_{n}=\left\langle G_{1}, \ldots, G_{n-1}, H_{n}\right\rangle$.

Proposition 5.4.1. For all $n \geq 0$, the group $H_{n}$ has Hausdorff dimension 1, and is branch.

Proof. We have the inclusion $\operatorname{Stab}_{H_{n}}\left(t_{n}\right) \leq X^{t_{n}} * L_{n+1}$. Also, using the fact that $G_{n}$ is self-similar, we have the inclusion

$$
\operatorname{Stab}_{H_{n}}\left(t_{n}+\cdots+t_{n+m}\right) \leq X^{t_{n}+\cdots+t_{n+m}} * L_{n+m+1}
$$

for all $m, n \geq 0$.

By Lemma 5.4.2, the inclusions $X^{t_{n}} * L_{n+1}^{\prime} \leq H_{n}^{\prime} \leq L_{n}^{\prime}$ hold for all $n \geq 0$. As a consequence we have

$$
X^{t_{n}+\cdots+t_{n+m}} * L_{n+m+1}^{\prime} \leq \operatorname{Rist}_{H_{n}}\left(t_{n}+\cdots+t_{n+m}\right)
$$


for all $m, n \geq 0$.

Finally, $L_{n}$ is generated by finitely many involutions, so that the index $\left[L_{n}\right.$ : $\left.L_{n}^{\prime}\right]$ is finite. Using Equation (5.4.1), we deduce that the inclusions

$$
\begin{aligned}
X^{t_{n}+\cdots+t_{n+m}} * L_{n+m+1}^{\prime} \leq \operatorname{Rist}_{H_{n}}\left(t_{n}+\cdots+\right. & \left.t_{n+m}\right) \\
& \leq \operatorname{Stab}_{H_{n}}\left(t_{n}+\cdots+t_{n+m}\right) \leq H_{n}
\end{aligned}
$$

all have finite index, and so $H_{n}$ is branch. Because $L_{n}^{\prime}$ contains $G_{n}^{\prime}$, this also proves the equality $\operatorname{dim}_{H} H_{n}=1$.

Lemma 5.4.2. Write $H=\left\langle a, d, \delta\left(a, d, g_{3}, \ldots, g_{t}\right)\right\rangle$ and $L=\left\langle a, d, g_{3}, \ldots, g_{t}\right\rangle$. The inclusion $X^{t} * L^{\prime} \leq H^{\prime}$ holds.

Proof. Write $g_{1}=a$ and $g_{2}=d$. For any $n \in \mathbb{Z}$, we have

$$
\delta\left(g_{1}, \ldots, g_{t}\right)^{(a d)^{n}}=\prod_{i=0}^{t-1}\left(\underline{1}^{i} \underline{0}^{t-i}\right)^{(a d)^{n}} * g_{i+1}^{s_{i+1}}
$$

where $s_{i}$ is an element in $\langle a, d\rangle$. The element $a d$ induces a full cycle on $X^{t}$, and for any pair $i \neq j$, we can find $n$ so that

$$
\left[\delta\left(g_{1}, \ldots, g_{t}\right)^{(a d)^{n}}, \delta\left(g_{1}, \ldots, g_{t}\right)\right]=v *\left[g_{i}^{s}, g_{j}\right]
$$

for some $v \in X^{t}$ and $s \in\langle a, d\rangle$. Notice that $\langle a, d\rangle$ is an infinite dihedral group, and consider the element $x=(a d)^{2^{t}}$. It is easy to see that $x$ fixes the $t$-th level, and acts almost diagonally: $x @ v$ is in $\{a d, d a\}$ for all $v \in X^{t}$. Thus in Equation (5.4.3), one can vary $n$ to choose $s$ as one wishes in the transversal $s\langle a d\rangle$. In particular, for all $j$, the group $H^{\prime}$ contains $v *\left[a, g_{j}\right]$ and $v^{\prime} *\left[d, g_{j}\right]$ for some $v, v^{\prime} \in X^{t}$. Conjugating these elements again by an appropriate power of $a d$, we deduce that $H^{\prime}$ contains $v *\left[a, g_{j}\right]$ and $v *\left[d, g_{j}\right]$ for all $v \in X^{t}$.

Looking back at Equation (5.4.2), we deduce

$$
\delta\left(g_{1}, \ldots, g_{t}\right)^{(a d)^{n}} \equiv \prod_{i=0}^{t-1}\left(\underline{1}^{i} \underline{0}^{t-i}\right)^{(a d)^{n}} * g_{i+1}
$$

modulo terms in $H^{\prime}$. Therefore, from Equation (5.4.3), we have

$$
\left[\delta\left(g_{1}, \ldots, g_{t}\right)^{(a d)^{n}}, \delta\left(g_{1}, \ldots, g_{t}\right)\right] \equiv v *\left[g_{i}, g_{j}\right]
$$

modulo terms in $H^{\prime}$. Using again conjugation by $a d$, we see that $H^{\prime}$ contains $v *\left[g_{i}, g_{j}\right]$ for all $v \in X^{t}$. Moreover, by Equation (5.4.4), the group $v^{-1} * H^{\prime}$ is normal in $L$. Thus $v^{-1} * H^{\prime}$ contains $L^{\prime}$ and we have $X^{t} * L^{\prime} \leq H^{\prime}$. 



\section{Chapter 6}

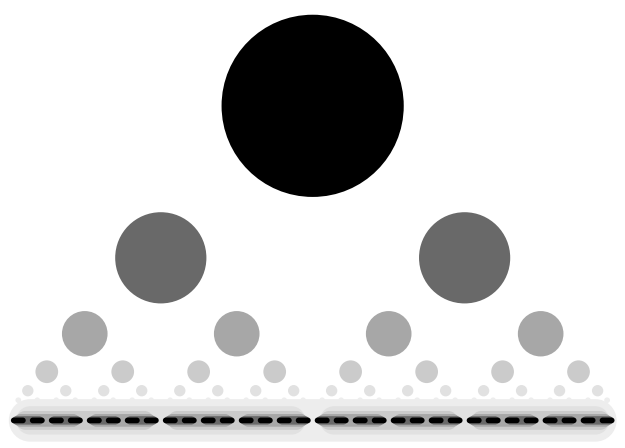

\section{Congruence Problems}

This small chapter is an addendum to [BSZ09], which is reprinted in Appendix $\mathrm{C}$. The original article is written in a language and with a notation very close to this thesis.

For regular branch groups, the main structure result describes the branch kernel:

Corollary C.2.10. Let $\Gamma$ be a level-transitive, self-similar, regular branch group and let $K$ be its maximal branching subgroup. The branch kernel $\mathcal{K}=\operatorname{ker}(\widehat{\Gamma} \rightarrow \widetilde{\Gamma})$ is isomorphic to $\widehat{\mathbb{Z}}[[\widetilde{\Gamma}]] \widehat{\otimes}_{\left.\widehat{\mathbb{Z}}_{[}\left[\widetilde{\Gamma}_{w}\right]\right]}$ A where $A$ is the inverse limit of $\left\{K /\left(K^{\prime} K^{e}\right)\right\}$ with the maps induced by the composition

$$
K / K^{\prime} \simeq\left(K / K^{\prime}\right) \times\{1\} \times \cdots \times\{1\} \rightarrow\left(K / K^{\prime}\right)^{d} \rightarrow K /\left(K^{\prime}\right)^{d} \rightarrow K / K^{\prime} .
$$

In particular, if $K / K^{\prime}$ has finite exponent dividing e, then the branch kernel has exponent dividing $e$.

We see that the branch kernel consists of copies of a (profinite) Abelian group attached to the boundary of the tree, in a profinite fashion. The highlight of this chapter is Theorem 6.1.3, which explains the structure of the rigid kernel. It shows that up to finite index, the rigid kernel is an inverse limit of copies of a finite group $\mathcal{K}_{1}$ attached to each vertex of the tree, where each copy of $\mathcal{K}_{1}$ acts on the copies located at descendant vertices.

In Section 6.2 we describe a group which has infinite rigid kernel.

\subsection{Structure of the Rigid Kernel}

Here is a simple observation, first.

Proposition 6.1.1. Let $G$ be a level-transitive, self-similar group, regular branch over a normal subgroup $K$. If $G / K$ is Abelian or elementary Abelian, then so is the rigid kernel of $G$.

Proof. The rigid kernel of $G$ can be computed as $\lim _{n} \operatorname{Stab}_{G}(n) /\left(X^{n} * K\right)$ by Corollary C.1.7. Using self-similarity, the group $\operatorname{Stab}_{G}(n) /\left(X^{n} * K\right)$ embeds into $X^{n} *(G / K)$. 


\subsection{Structure of the Rigid Kernel}

Remark 6.1.2. The assumption that $K$ be normal is not restrictive. Indeed, if $K$ is the maximal branching subgroup of $G$, then $K$ contains $\operatorname{Rist}_{G}(n)$ for some $n$ by Corollary C.1.7. Therefore, $\operatorname{Core}_{G}(K)$, the intersection of all $G$-conjugates of $K$, also contains $\operatorname{Rist}_{G}(n)$, and it is easy to show that $\operatorname{Core}_{G}(K)$ is again a branching subgroup.

Theorem 6.1.3. Let $G$ be a level-transitive, self-similar group, regular branch over a normal subgroup $K$. The rigid kernel of $G$ is a finite extension of a profinite group $\mathcal{K}_{\infty}=\lim _{\leftarrow} \mathcal{K}_{n}$, where $\mathcal{K}_{n}=\lim _{m}\left(\operatorname{Stab}_{K}(m)\left(X^{n} * K\right)\right) /\left(X^{n} * K\right)$ is a finite group and the connecting map $\mathcal{K}_{m} \rightarrow \mathcal{K}_{n}$ yields an exact sequence

$$
1 \rightarrow X^{n} * \mathcal{K}_{m-n} \rightarrow \mathcal{K}_{m} \rightarrow \mathcal{K}_{n} \rightarrow 1
$$

Also, the action of $\mathcal{K}_{m}$ preserves each copy of $\mathcal{K}_{m-n}$. Moreover, the canonical maps $\mathcal{K}_{\infty} \rightarrow \mathcal{K}_{n}$ satisfy the short exact sequence of profinite groups

$$
1 \rightarrow X^{n} * \mathcal{K}_{\infty} \rightarrow \mathcal{K}_{\infty} \rightarrow \mathcal{K}_{n} \rightarrow 1
$$

and the action of $\mathcal{K}_{\infty}$ is continuous and preserves each copy of $\mathcal{K}_{\infty}$.

Corollary 6.1.4. The rigid kernel of $G$ is finite if and only if $X * K$ is a congruence subgroup in $K$.

Proof. Using the short exact sequence several times, we see that $\mathcal{K}_{n}$ is an extension of copies of $\mathcal{K}_{1}$. In particular the order of $\mathcal{K}_{n}$ is $\left|\mathcal{K}_{1}\right|$ raised to the power $\frac{|X|^{n}-1}{|X|-1}$. Thus $\mathcal{K}_{\infty}$ is infinite if and only if $\mathcal{K}_{1}$ is non-trivial. This happens exactly when $X * K$ is not a congruence subgroup in $K$.

Corollary 6.1.5. If the action is trivial in every exact sequence $1 \rightarrow X^{n} *$ $\mathcal{K}_{m-n} \rightarrow \mathcal{K}_{m} \rightarrow \mathcal{K}_{n} \rightarrow 1$, then we have

$$
\mathcal{K}_{\infty}=\lim _{n} \prod_{\substack{v \in X^{*} \\|v|<n}} v * \mathcal{K}_{1} .
$$

Proof. By induction, the equality $\mathcal{K}_{n}=\prod_{\substack{v \in X^{*} \\|v|<n}}\left(v * \mathcal{K}_{1}\right)$ holds.

Proof of Theorem 6.1.3. By Corollary C.1.7, the filters $\left\{X^{n} * K \mid n \geq 0\right\}$ and $\left\{\operatorname{Rist}_{G}(n) \mid n \geq 0\right\}$ are cofinal. Thus the rigid kernel of $G$ can be computed as $\varliminf_{n} \operatorname{Stab}_{G}(n) /\left(X^{n} * K\right)$. Note that this is an extension of $\varliminf_{n} \operatorname{Stab}_{K}(n) /\left(X^{n} * K\right)$ by $\lim _{n} \operatorname{Stab}_{G}(n) / \operatorname{Stab}_{K}(n)$, the latter being a finite group of order at most $\left[G: \overleftarrow{K}^{n}\right.$.

We now prove the claim about the group

$$
\mathcal{K}_{\infty}={\underset{n}{n}}_{\lim _{n}} \operatorname{Stab}_{K}(n) /\left(X^{n} * K\right)={\underset{\leftrightarrows}{m, n}}_{\lim }\left(\operatorname{Stab}_{K}(m)\left(X^{n} * K\right)\right) /\left(X^{n} * K\right) .
$$

Define $\mathcal{K}_{n}=\lim _{\longleftarrow}\left(\operatorname{Stab}_{K}(m)\left(X^{n} * K\right)\right) /\left(X^{n} * K\right)$, so that we have $\mathcal{K}_{\infty}=\lim _{n} \mathcal{K}_{n}$. These groups are shown on Figure 6.1. We prove that the groups $\mathcal{K}_{m}$ satisfy the recurrence relation

$$
1 \rightarrow X^{n} * \mathcal{K}_{m-n} \rightarrow \mathcal{K}_{m} \rightarrow \mathcal{K}_{n} \rightarrow 1
$$




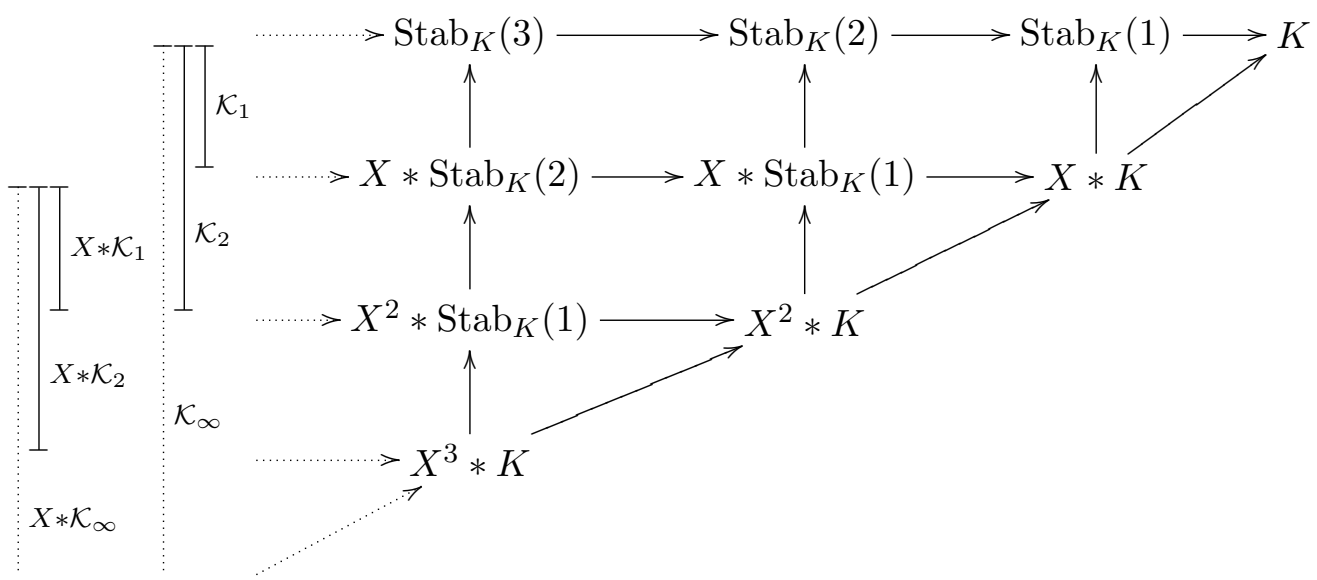

Figure 6.1: Some of the groups involved in the proof of Theorem 6.1.3. All the maps are inclusions.

Set $m \geq n \geq 0$ and consider the intersection of $\mathcal{K}_{m}$ with $\left(X^{n} * K\right) /\left(X^{m} * K\right)$. This is precisely $\lim _{l}\left(\left(X^{n} * \operatorname{Stab}_{K}(l-n)\right)\left(X^{m} * K\right)\right) /\left(X^{m} * K\right)=X^{n} * \mathcal{K}_{m-n}$. Moreover, $X^{n} * \mathcal{K}_{m-n}$ is normal in $\mathcal{K}_{m}$, because $X^{n} * K$ is normal in $K$. The quotient $\mathcal{K}_{m} /\left(X^{n} * \mathcal{K}_{m-n}\right)$ is isomorphic to $\lim _{l}\left(\operatorname{Stab}_{K}(l)\left(X^{n} * K\right)\right) /\left(X^{n} * K\right)$; this is $\mathcal{K}_{n}$ by definition. It is clear from the definition of $\mathcal{K}_{m}$ that its action must preserve each copy of $\mathcal{K}_{m-n}$.

Inverse limit of the exact sequences yields the claims about $\mathcal{K}_{\infty}$.

\subsection{An Example}

Set $X=\{1,2,3,4\}$ and consider the following tree automorphisms:

$$
\begin{array}{ll}
a_{1}=\left(a_{1}, 1,1,1\right) \sigma_{234}, & a_{2}=\left(1, a_{2}, 1,1\right) \sigma_{341}, \\
a_{3}=\left(1,1, a_{3}, 1\right) \sigma_{412}, & a_{4}=\left(1,1,1, a_{4}\right) \sigma_{123},
\end{array}
$$

where $\sigma_{i j k}$ cyclically permutes $i, j$ and $k$. We let $H$ be the group generated by $a_{1}, a_{2}, a_{3}, a_{4}$. We note that the automorphism $s=(s, s, s, s) \sigma_{1234}$, which cyclically permutes the letters of every word in $X^{*}$, permutes the generators of $H$, and is thus an automorphism of $H$. It is an outer automorphism, since the induced automorphism on $\pi_{1}(H)=\operatorname{Alt}(4)$ is outer.

Clearly $H$ is self-similar. It is also level-transitive and recurrent (that is, $\operatorname{Stab}_{H}(x) @ x=H$ for all $\left.x \in X\right)$. Moreover, $H$ is contracting, and using "germs" (as in Section D.6 for example), it is not hard to see that the Abelianization of $H$ is isomorphic to $(\mathbb{Z} / 3 \mathbb{Z})^{4}$.

Proposition 6.2.1. The group $H$ is regular branch over $K=[H, H]$. 


\subsection{An Example}

Proof. $K$ has finite index in $H$, and since $H$ is self-similar, it is enough to show that $K$ is branching.

Let $S$ be a set which generates $K$ as a normal subgroup of $H$. Since $H$ is leveltransitive and recurrent, it is enough to show that for all $s \in S, K$ contains $x * t$ for some $x \in X$ and some conjugate $t$ of $s$. Moreover, using the automorphism permuting the generators of $H$, it is enough to show that this holds for $\left[a_{1}, a_{2}\right]$ and $\left[a_{1}, a_{3}\right]$. We have

$$
\begin{aligned}
& {\left[a_{3}^{-a_{1}}, a_{3}^{-a_{2}}\right] \cdot\left(a_{2}^{-1} a_{1}\right)^{3}=3 *\left(\left[a_{1}, a_{2}\right]^{a_{2}}\right),} \\
& {\left[a_{2}^{a_{1}^{-1}}, a_{2}^{a_{3}}\right] \cdot\left(a_{1} a_{3}\right)^{-3}=2 *\left(\left[a_{1}, a_{3}\right]^{-a_{3}^{-1}}\right) .}
\end{aligned}
$$

Thus the proposition holds.

Lemma 6.2.2. The group $K$ contains $\left(a_{i}, 1,1, a_{i}^{-1}\right)$ for all $i \in\{1,2,3,4\}$.

Proof. We compute

$$
\left[a_{3}^{a_{1}}, a_{4}\right]=\left(a_{1}, a_{1}, a_{1},\left[a_{3}, a_{4}\right]\right) \equiv\left(a_{1}, a_{1}, a_{1}, 1\right) \quad(\bmod X * K) .
$$

Since $K$ contains $X * K$, this proves $\left(a_{1}, a_{1}, a_{1}, 1\right)$ is in $K$. Conjugating this element by $a_{2}$, we get $\left(1, a_{1}, a_{1}, a_{1}\right)$, modulo $X * K$. Therefore $\left(a_{1}, 1,1, a_{1}^{-1}\right)$ is in $K$. Using the automorphism which permutes the generators of $H$ and conjugation in $H$, we see that $K$ contains in fact $\left(x * a_{i}\right)\left(y * a_{i}^{-1}\right)$ for all $x, y \in X$ and $i \in\{1,2,3,4\}$.

Theorem 6.2.3. The rigid kernel of $H$ is $(\mathbb{Z} / 3 \mathbb{Z})^{\infty}$.

Proof. The quotient $H / K$ is an elementary Abelian 3-group, therefore so is the rigid kernel of $H$ by Proposition 6.1.1. To prove that it is infinite, it is enough to

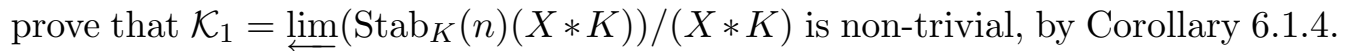

Set $\beta=a_{1} a_{2} a_{3} a_{4}$ and $\alpha=\left(\beta, 1,1, \beta^{-1}\right)$. Using Lemma 6.2 .2 and the fact that $K$ is branching, we see that the element $\alpha$ is in $K$. Moreover, the image of $\beta$ in $H / K$ is non-trivial, therefore the image of $\alpha$ is non-trivial in $K /(X * K)$. We now prove that for all $n \geq 0, \alpha$ is congruent to an element of $\operatorname{Stab}_{K}(n)$ modulo $X * K$. For this it is sufficient to prove that for all $n \geq 0, \beta$ is congruent to an element of $\operatorname{Stab}_{H}(n)$ modulo $K$. Using Lemma 6.2.2 again, we compute

$$
\beta=\left(a_{1} a_{3}, 1,1, a_{2} a_{4}\right) \equiv\left(a_{1} a_{2} a_{3} a_{4}, 1,1,1\right)=1 * \beta \quad(\bmod K) .
$$

This proves that $\mathcal{K}_{1}$ contains $\mathbb{Z} / 3 \mathbb{Z}$; in particular $\mathcal{K}_{1}$ is non-trivial. 


\section{Chapter 7}

\section{Twisted Twins}

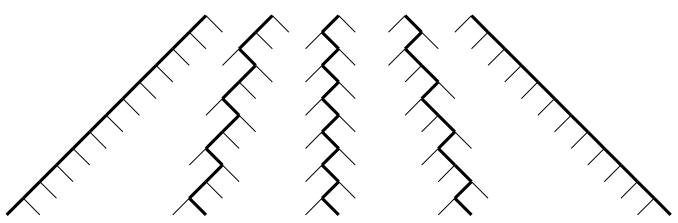

In [BS09] we studied "the" twisted twin of the Grigorchuk group. It is in fact the nicest of a huge collection of twins, which are all contained in one big group $\mathrm{G}_{2}$. The present chapter is dedicated to this group.

In Section 7.1 we define $G_{2}$ as the spinal subgroup of a particular closed subgroup of Aut $X^{*}$. We summarize the results from Section 2.8 which apply to this group, and some results concerning the order of elements in the closure of the Grigorchuk group are given as well. In Section 7.2 we prove $G_{2}$ is generated by its 1-spinal elements. We also prove that $G_{2}$ is regular branch. Finally we give a presentation of $G_{2}$ in Section 7.3. As a corollary we show that the Abelianization of this group has infinite rank.

We consider $p=2$, and $k=\mathbb{F}_{2}=X$. For $x \in X$, we write $\bar{x}=1-x$.

\subsection{The Closure of the Grigorchuk Group}

Let G be the "first Grigorchuk group", see Section A.1 for the definition. It is established in [Gri00, Proposition 10] that $\mathrm{G}$ has the congruence property (any finite subgroup of $\mathrm{G}$ contains $\operatorname{Stab}_{\mathrm{G}}(n)$ for some $n$ large enough). Hence the profinite completion of $\mathrm{G}$ coincides with its closure $\overline{\mathrm{G}}$ in Aut $X^{*}$. Since $\overline{\mathrm{G}}$ is selfsimilar and regular branch, this group is easily described in terms of equations. Write

$$
\begin{aligned}
P_{\Delta} & =\left[\Delta^{1}+\Delta^{2}+\Delta^{3}\right], \\
P_{0} & =\left[\underline{0}+\underline{1} *\left(\Delta^{1}+\Delta^{2}\right)\right], \\
Q & =[\underline{0}] \cdot[\underline{1}]+\left[\Delta^{1} * \underline{1}+\Delta^{1} * \underline{0} * \Delta^{1}\right] .
\end{aligned}
$$

In Proposition A.1.1 we prove the equality

$$
\overline{\mathrm{G}}=\mathcal{V}\left(\left\{v * P_{\Delta}, v * P_{0}, v * Q \mid v \in X^{*}\right\}\right) .
$$

We define the group $\overline{\mathrm{G}_{2}}$ as

$$
\overline{\mathrm{G}_{2}}=\mathcal{V}\left(\left\{v * P_{\Delta}, v * P_{0} \mid v \in X^{*}\right\}\right) .
$$




\subsection{The Closure of the Grigorchuk Group}

Obviously $\bar{G}$ is a subgroup of $\overline{G_{2}}$. We let $G_{2}$ denote the bounded subgroup of $\overline{G_{2}}$. The group $\overline{\mathrm{G}_{2}}$ satisfies the hypotheses needed to apply the various propositions in Section 2.8. We summarize some consequences:

Fact 1. The finitary subgroup of $\overline{G_{2}}$ is the cyclic group of order 2 generated by a.

Fact 2. Suppose $g \in \overline{\mathrm{G}_{2}}$ is active at the vertex $v x$ for some $v \in X^{*}, x \in X$. Then $g @ v \bar{x}$ is not finitary.

Fact 3. The bounded subgroup $G_{2}$ of $\overline{G_{2}}$ coincides with the spinal subgroup of $\overline{\mathrm{G}_{2}}$.

Fact 4. Every element of $G_{2}$ has finite order.

Also, as a topological group, $\overline{\mathrm{G}_{2}}$ is not finitely generated (see Example 2.2.10). It is striking that the torsion property is not specific to $G$, but is shared by all the elements of $G_{2}$. We note that there are elements in $\bar{G}$ which have finite order, but are not in $\mathrm{G}_{2}$ :

Example 7.1.1. We construct an automorphism $t \in \overline{\mathrm{G}}$ which is not spinal, and thus not in $\mathrm{G}_{2}$. Set $x=[a, b]$. It is easy to see that $x$ is 2 -spinal and of order 8 . Since $x$ is in the branching subgroup of $\mathrm{G}$, the element $t=(x, t)$ belongs to $\overline{\mathrm{G}}$, is of order 8 , but is not spinal.

Recall that $g \in$ Aut $X^{*}$ is finite-state if $\left\{g @ v \mid v \in X^{*}\right\}$ is finite.

Proposition 7.1.2. There is $g \in \overline{\mathrm{G}}$ which is finite-state, and has infinite order.

Proof. Write $x=[a, b]$ and define $x_{0}=x$ and $x_{n+1}=\left(011110 * x_{n}\right) x$ for $n \geq 0$. We define $x_{\infty}$ as the only element which satisfies $x_{\infty}=\left(011110 * x_{\infty}\right) x$. The automorphism $x_{\infty}$ is finite-state as can be seen on Figure 7.1.

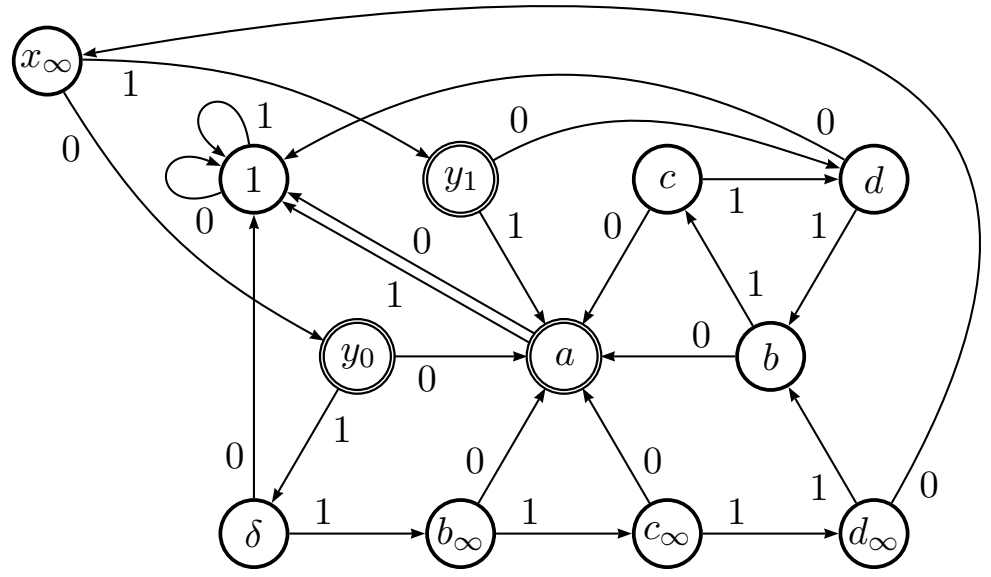

Figure 7.1: The finite automaton describing $x_{\infty}$. The states $y_{0}, y_{1}$ and $a$ are the only active ones. 
$x_{\infty}$ is in $\overline{\mathrm{G}}$ because each $x_{n}$ belongs to the branching subgroup $K$ of $\mathrm{G}$, and $x_{\infty}=\lim _{n \rightarrow \infty} x_{n}$ holds in Aut $X^{*}$. A straightforward computation shows that $\left(x_{\infty}\right)^{4}$ is in $\operatorname{Stab}(3)$, and $\left(\left(x_{\infty}\right)^{4}\right) @ 0 v=b_{\infty}$ for all $v \in X^{2}$. On the other hand, $b_{\infty}$ and $x_{\infty}$ have the same order because we have $\left(b_{\infty}\right) @ 110=x_{\infty}$ and $b_{\infty}$ fixes 110. Therefore $x_{\infty}$ has infinite order.

Remark 7.1.3. The automorphisms $t$ from Example 7.1 .1 and $x_{\infty}$ above share something in common. They are both finite-state, not bounded, but of growth degree 1 . This means that the number of non-trivial states at level $n$ grows linearly with $n$. It would be interesting to know whether one can add some automorphisms of degree 1 to $G_{2}$, so that the resulting group still be torsion.

\subsection{Generators and Branchness of $\mathrm{G}_{2}$}

Consider $w \in X^{\omega}$ and $x \in X$. We define the elements $b_{w}, c_{w}$ and $d_{w}$ as follows.

$b_{x w}=\left\{\begin{array}{ll}\left(c_{w}, a\right) & \text { if } x=0, \\ \left(a, c_{w}\right) & \text { if } x=1,\end{array} \quad c_{x w}=\left\{\begin{array}{ll}\left(d_{w}, a\right) & \text { if } x=0, \\ \left(a, d_{w}\right) & \text { if } x=1,\end{array} \quad d_{x w}= \begin{cases}\left(b_{w}, 1\right) & \text { if } x=0 \\ \left(1, b_{w}\right) & \text { if } x=1\end{cases}\right.\right.$

Let $g$ be one of the letters $b, c, d$. Then $g_{w}$ is a 1-spinal element, with spine $w$. Every $g_{w}$ has order 2, and the relation $g_{x w}=\left(g_{\bar{x} w}\right)^{a}$ holds for all $x \in X, w \in X^{\omega}$. Moreover $b_{w} c_{w} d_{w}=1$ for all $w \in X^{\omega}$. Also, with $w=1^{\infty}$, the automorphisms $b_{w}, c_{w}, d_{w}$ are the usual generators of the Grigorchuk group.

Lemma 7.2.1. The set of 1-spinal elements of $\overline{\mathrm{G}_{2}}$ is $\left\{b_{w}, c_{w}, d_{w} \mid w \in X^{\omega}\right\} \cdot\{1, a\}$.

Proof. Let $g \in \overline{\mathrm{G}_{2}}$ be a 1-spinal element, and let $s \in X^{\omega}$ be its spine.

First, note that $g$ has no activity outside the 1-neighbourhood of $s$. Indeed, due to Fact 1, $g @ v$ is in $\langle a\rangle$ for all $v \in X^{*} \backslash \operatorname{Prefix}(s)$. Also, $g$ has no activity along its spine, except maybe at $\varnothing$. This follows immediately from Fact 2.

Next, if $v \in X^{*}$ and $x, y, z \in X$ are such that $v x y z<s$, then among $g @ v \bar{x}$, $g @ v x \bar{y}$ and $g @ v x y \bar{z}$, exactly one is the identity, and the other two are equal to $a$. Indeed $g @ v \bar{x}, g @ v x \bar{y}$ and $g @ v x y \bar{z}$ are all in $\langle a\rangle$. Because $g$ satisfies $v * P_{\Delta}$, and $g$ has no activity along $s$, we have $([v \bar{x}]+[v x \bar{y}]+[v x y \bar{z}])(g)=0$. Hence either $[v \bar{x}](g),[v x \bar{y}](g)$ and $[v x y \bar{z}](g)$ are all zero, but in this case $g$ is easily seen to be finitary, or exactly one of $[v \bar{x}](g),[v x \bar{y}](g),[v x y \bar{z}](g)$ is zero.

The elements in the claim are the only remaining possibilities.

Lemma 7.2.2. The set of 1-spinal elements of $\overline{\mathrm{G}}$ is $\{1, a\} \cdot\left\{b_{w}, c_{11 w}, d_{1 w}\right\} \cdot\{1, a\}$ where $w$ ranges over the words which can be expressed as $w=x_{1} 11 x_{2} 11 x_{3} 11 \cdots$ for some $x_{i} \in X$.

Proof. Since $\overline{\mathrm{G}}$ is a subgroup of $\overline{\mathrm{G}_{2}}$, the set of 1 -spinal elements of $\overline{\mathrm{G}}$ must be a subset of $T=\left\{b_{w}, c_{w}, d_{w} \mid w \in X^{\omega}\right\} \cdot\{1, a\}$ by the previous lemma. Comparing Equations (7.1.1) and (7.1.2), we only need to select the elements of $T$ which satisfy $v * Q$ for all $v \in X^{*}$. 
7.2. Generators and Branchness of $\mathrm{G}_{2}$

A direct computation shows

$$
\begin{aligned}
& Q\left(b_{x y w}\right)=\bar{y}, \quad(x * Q)\left(b_{x y z w}\right)=\bar{z}, \quad(x y * Q)\left(b_{x y z t w}\right)=0, \\
& Q\left(c_{x y w}\right)=\bar{y}, \quad(x * Q)\left(c_{x y z w}\right)=0, \quad(x y * Q)\left(c_{x y z t w}\right)=\bar{t}, \\
& Q\left(d_{x y w}\right)=0, \quad(x * Q)\left(d_{x y z w}\right)=\bar{z}, \quad(x y * Q)\left(d_{x y z t w}\right)=\bar{t},
\end{aligned}
$$

for all $x, y, z, t \in X$ and $w \in X^{\omega}$. Hence we see that we must keep exactly the elements $\left\{b_{x 11 w}, c_{x 1 w}, d_{x w}\right\} \cdot\{1, a\}$ where $w$ can be expressed as $w=x_{1} 11 x_{2} 11 \cdots$ for some $x_{i} \in X$. The claim is just a reformulation, using the equality $g_{x w}^{a}=g_{\bar{x} w}$ for $g \in\{b, c, d\}$.

Theorem 7.2.3. $\mathrm{G}_{2}$ is generated by its 1-spinal elements, and is regular branch.

We split the theorem into two propositions. We define the groups

$$
\begin{aligned}
& H=\left\langle a, b_{w}, c_{w}, d_{w} \mid w \in X^{\omega}\right\rangle \leq \mathrm{G}_{2}, \\
& K=\left\langle\left[a, b_{w}\right],\left[b_{w}, c_{w^{\prime}}\right],\left[b_{w}, d_{w^{\prime}}\right],\left[c_{w}, d_{w^{\prime}}\right], b_{w} c_{w^{\prime}} d_{w^{\prime \prime}} \mid w, w^{\prime}, w^{\prime \prime} \in X^{\omega}\right\rangle^{H},
\end{aligned}
$$

where the superscript stands for normal closure in $H$.

Proposition 7.2.4. The group $H$ is regular branch over $K$.

Proposition 7.2.5. The group $\mathrm{G}_{2}$ coincides with $H$.

These propositions together imply Theorem 7.2.3.

Proof of Proposition 7.2.4. Let $B \leq H$ be the subgroup $\left\langle b_{w}, c_{w} d_{w^{\prime}}\right| w, w^{\prime} \in$ $\left.X^{\omega}\right\rangle^{H}$ and fix $w_{0} \in X^{\omega}$. Then $H / B$ is generated by the images of $a, d_{w_{0}}$. Since $\left\langle a, d_{w_{0}}\right\rangle$ is a dihedral group of order 8 , it follows that $B$ has index at most 8 in $H$.

Now $B / K$ is generated by the image of $b_{w_{0}}$, hence $K$ is of index at most 2 in $B$, and at most 16 in $H$. We prove that $K$ contains $K \times K$. Because $H$ is level-transitive and recurrent, it is sufficient to prove that $(1, x)$ is in $K$ for all $x$ which generate $K$ as a normal subgroup of $H$. Straightforward computations show that the relations

$$
\begin{aligned}
{\left[c_{0 w}, d_{1 w}\right] } & =\left(1,\left[a, b_{w}\right]\right), & & {\left[d_{1 w}, b_{1 w^{\prime}}\right]=\left(1,\left[b_{w}, c_{w^{\prime}}\right]\right), } \\
{\left[d_{1 w}, c_{1 w^{\prime}}\right] } & =\left(1,\left[b_{w}, d_{w^{\prime}}\right]\right), & & {\left[b_{1 w}, c_{1 w^{\prime}}\right]=\left(1,\left[c_{w}, d_{w^{\prime}}\right]\right), } \\
d_{1 w} b_{1 w^{\prime}} c_{1 w^{\prime \prime}} & =\left(1, b_{w} c_{w^{\prime}} d_{w^{\prime \prime}}\right), & &
\end{aligned}
$$

hold for all $w, w^{\prime}, w^{\prime \prime} \in X^{\omega}$. Hence $K \times K \leq K$.

We now show that $K \times K$ has finite index in $K$. From the equalities $d_{1 w}=$ $\left(1, b_{w}\right)$ and $b_{1 w} c_{1 w^{\prime}}=\left(1, c_{w} d_{w^{\prime}}\right)$ we see that $H$ contains $B \times B=\left\langle d_{1 w}, b_{1 w} c_{1 w^{\prime}}\right|$ $\left.w, w^{\prime} \in X^{\omega}\right\rangle^{H}$. The quotient $H /(B \times B)$ is generated by the images of $a$ and $c_{w_{0}}$, thus $B \times B$ has finite index in $H$. Therefore $K \times K$ has finite index in $H$, and a fortiori in $K$. 
We still need a few results before proving Proposition 7.2.5. Because of the equality $b_{0 w}^{a}=b_{1 w}$ (and similarly for $c_{w}$ and $d_{w}$ ), the group $H$ is generated by $\left\{a, b_{1 w}, c_{1 w}, d_{1 w} \mid w \in X^{\omega}\right\}$. We fix $w_{0} \in X^{\omega}$ and write $d=d_{w_{0}}$. Define the map $\varphi$ on this generating set by

$$
\begin{aligned}
& a \mapsto c_{0 w_{0}}=(d, a), \\
& c_{1 w} \mapsto b_{11 w}=\left(a, c_{1 w}\right), \\
& b_{1 w} \mapsto d_{11 w}=\left(1, b_{1 w}\right), \\
& d_{1 w} \mapsto c_{11 w}=\left(a, d_{1 w}\right) \text {, }
\end{aligned}
$$

for all $w \in X^{\omega}$.

Proposition 7.2.6. The map $\varphi$ extends to a homomorphism $H \rightarrow H$.

Proof. Consider the map $\varphi_{0}$, the projection $\varphi$ to the first factor:

$$
a \mapsto d, \quad b_{1 w} \mapsto 1, \quad c_{1 w} \mapsto a, \quad d_{1 w} \mapsto a .
$$

It is enough to show that $\varphi_{0}$ extends to a group homomorphism. The group generated by $a$ and $d$ is a dihedral group of order 8 , and it is easy to check that $\varphi_{0}$ factors through $\pi_{3}: H \rightarrow H / \operatorname{Stab}_{H}(3)$.

Let $D$ be the dihedral group of order 8 generated by $a$ and $d$. We note that for all $g \in H$ we have $\varphi(g)=(\delta, g)$ for some $\delta \in D$.

Lemma 7.2.7. If $g$ is an element of $\overline{\mathrm{G}_{2}}$ with $g @ 0 \in D$ and $g @ 1=1$, then $g$ belongs to $H$.

Proof. If $g$ belongs to $\overline{\mathrm{G}_{2}}$, then it must satisfy $P_{\Delta}$ and $P_{0}$. A simple check on the 8 elements of $D$ shows that only the identity and $d a d a$ are allowed for $g @ 0$. A straightforward computation shows $c_{0 w_{0}} c_{11 w} c_{0 w_{0}} c_{10 w}=\left(d_{a d a}, 1\right) \in H$.

Corollary 7.2.8. Suppose $g$ is an element of $\overline{\mathrm{G}_{2}}$ with $g @ x \in H$ for all $x \in X$. Then $g$ is in $H$.

Proof. Since $H$ contains $a$, we may suppose $g$ is not active at the root. We compute

$$
h=\varphi(g @ 0)^{-a} g \varphi(g @ 1)^{-1}=\left((g @ 0)^{-1}, \delta\right)(g @ 0, g @ 1)\left(\delta^{\prime},(g @ 1)^{-1}\right)=\left(\delta^{\prime}, \delta\right)
$$

for some $\delta, \delta^{\prime} \in D$. Since $D \leq H$ we further compute $\varphi(\delta)^{-1} h=\left(\delta^{\prime \prime}, 1\right)$ for some $\delta^{\prime \prime} \in D$. The lemma shows that $\left(\delta^{\prime \prime}, 1\right)$ belongs to $H$, and we conclude that $g$ is in $H$.

Proof of Proposition 7.2.5. It is enough to prove the inclusion $\mathrm{G}_{2} \leq H$.

Let $\tau: \mathrm{G}_{2} \rightarrow \mathbb{N} \times \mathbb{N}$ be the map $g \mapsto(k, n)$ where $k$ is the number of spines of $g$, and $n$ is 0 if $g$ is finitary or 1-spinal, or equal to the length of the maximal common prefix of the spines of $g$ if $k \geq 2$. We consider lexicographic ordering on $\mathbb{N} \times \mathbb{N}$ and proceed by induction on $\tau(g)$. The cases $\tau(g)=(0,0)$ and $\tau(g)=(1,0)$ hold by Fact 1 and Lemma 7.2.1. Up to multiplication by $a$ one can assume $[\varnothing](g)=0$.

Suppose $\tau(g)=(k, 0)$ for some $k \geq 2$. Then $\tau(g @ x)<(k, 0)$ for all $x \in$ $X$ and thus $g @ x$ is in $H$ by the induction hypothesis. Therefore $g$ is in $H$ by Corollary 7.2.8. Suppose now $\tau(g)=(k, n)$ for some $n>0$. Then up to conjugation by $a$ we may suppose $g @ 0$ is finitary, and we have $\tau(g @ 1)=(k, n-1)$. Therefore $g @ 1$ belongs to $H$ by the induction hypothesis, and so $g$ is in $H$ by Corollary 7.2.8. 


\subsection{A Presentation of $\mathrm{G}_{2}$}

\subsection{A Presentation of $\mathrm{G}_{2}$}

Using the method described in [Bar03], we provide a presentation of $\mathrm{G}_{2}$. Write $c=c_{11 w_{0}}$ and $\bar{c}=c_{10 w_{0}}$.

Theorem 7.3.1. Let $w_{0}$ be any point of $X^{\omega}$. The group $\mathrm{G}_{2}$ admits the $L$ presentation

$$
\begin{aligned}
& \mathrm{G}_{2}=\left\langle a, b_{w}, c_{w}, d_{w}|\tilde{\varphi}| a^{2}, b_{w}^{2}, c_{w}^{2}, d_{w}^{2}, b_{w} c_{w} d_{w},\right. \\
& {\left[d_{1 w}^{a}, d_{1 w^{\prime}}^{t}\right],\left[d_{1 w}^{a},\left(c \beta^{\prime}\right)^{t}\right],\left[(c \beta)^{a},\left(c \beta^{\prime}\right)^{t}\right], b_{0 w}^{a} b_{1 w}, c_{0 w}^{a} c_{1 w}, d_{0 w}^{a} d_{1 w},} \\
& \left.c^{a} b_{10 w} \bar{c}^{a} b_{11 w}, \bar{c}^{a} b_{10 w} c^{a} b_{11 w}, c^{a} c_{10 w} \bar{c}^{a} c_{11 w}, \bar{c}^{a} c_{10 w} c^{a} c_{11 w}, d_{10 w}^{c^{a}} d_{11 w}\right\rangle,
\end{aligned}
$$

where $\tilde{\varphi}$ is the endomorphism of the free group on $\left\{a, b_{w}, c_{w}, d_{w}\right\}$ induced by

$$
\begin{aligned}
& a \mapsto c^{a}, \quad b_{1 w} \mapsto d_{11 w}, \quad c_{1 w} \mapsto b_{11 w}, \quad d_{1 w} \mapsto c_{11 w}, \\
& b_{0 w} \mapsto c^{a} d_{11 w} c^{a}, \quad c_{0 w} \mapsto c^{a} b_{11 w} c^{a}, \quad d_{0 w} \mapsto c^{a} c_{11 w} c^{a} .
\end{aligned}
$$

In the above $w$ and $w^{\prime}$ range over $X^{\omega} ; t$ over $\left\{1, c, c^{a}, c c^{a}\right\} ; \beta$ over $\left\{b_{1 w}, c_{1 w}\right\} ; \beta^{\prime}$ over $\left\{b_{1 w^{\prime}}, c_{1 w^{\prime}}\right\}$.

Corollary 7.3.2. The Abelianization of $\mathrm{G}_{2}$ is $(\mathbb{Z} / 2 \mathbb{Z})^{\infty}$.

Proof. $\mathrm{G}_{2}$ is generated by involutions, hence $\mathrm{G}_{2} / \mathrm{G}_{2}{ }^{\prime}$ is an elementary Abelian 2 -group. It is easy to get an $L$-presentation for the Abelianization of $G_{2}$, as a $\mathbb{Z} / 2 \mathbb{Z}$-module:

$$
\begin{array}{r}
\mathrm{G}_{2} / \mathrm{G}_{2}{ }^{\prime}=\left\langle a, b_{w}, c_{w}, d_{w}|\widehat{\varphi}| b_{w}+c_{w}+d_{w}, b_{0 w}+b_{1 w}, c_{0 w}+c_{1 w}, d_{0 w}+d_{1 w},\right. \\
\left.b_{10 w}+b_{11 w}+c+\bar{c}, c_{10 w}+c_{11 w}+c+\bar{c}, d_{10 w}+d_{11 w}\right\rangle
\end{array}
$$

where $\widehat{\varphi}$ is the endomorphism of the free Abelian group on $\left\{a, b_{w}, c_{w}, d_{w}\right\}$ induced by

$$
a \mapsto c, \quad b_{w} \mapsto d_{1 w}, \quad c_{w} \mapsto b_{1 w}, \quad d_{w} \mapsto c_{1 w},
$$

for all $w, w^{\prime} \in X^{\omega}$.

The last few relations are consequences of the first ones via $\widehat{\varphi}$, so we can rewrite this presentation as

$$
\mathrm{G}_{2} / \mathrm{G}_{2}{ }^{\prime}=\left\langle a, b_{w}, c_{w}, d_{w}|\widehat{\varphi}| b_{w}+c_{w}+d_{w}, b_{0 w}+b_{1 w}, c_{0 w}+c_{1 w}, d_{0 w}+d_{1 w}\right\rangle .
$$

Using $\widehat{\varphi}$, we see that $b_{w}$ is equal to $b_{w^{\prime}}$ if and only if $w$ and $w^{\prime}$ are cofinal, and similarly for $c_{w}$ and $d_{w}$. Therefore we need the generator $a$, and two generators for each cofinality class of $X^{\omega}$. Thus the group $\mathrm{G}_{2} / \mathrm{G}_{2}{ }^{\prime}$ is not even countably generated.

It is immediate that the restriction of $\varphi$ to $K$ sends $x$ to $(1, x)$. Before proving Theorem 7.3.1, we need some preliminary results. 
Definition 7.3.3. Let $G \leq$ Aut $X^{*}$ be a self-similar group. The contraction nucleus, or simply the nucleus of $G$ is the minimal set $N \subseteq G$ such that for every $g \in G$ there is an integer $n$ such that $g @ w \in N$ for all $w \in X^{*}$ of length at least $n$.

Lemma 7.3.4. The nucleus of $\mathrm{G}_{2}$ is $N=\left\{1, a, b_{w}, c_{w}, d_{w} \mid w \in X^{\omega}\right\}$.

Proof. We know that $N$ generates $\mathrm{G}_{2}$. Since $\mathrm{G}_{2}$ consists of spinal automorphisms, the elements of its nucleus are at most 1-spinal. Also, $N$ is clearly minimal.

We recall the general strategy in obtaining presentations by generators and relations for self-similar groups; for more details see [Bar03] or [Sid87].

The only relations of length $\leq 3$ among elements of $N$ are $a^{2}=b_{w}^{2}=c_{w}^{2}=$ $d_{w}^{2}=b_{w} c_{w} d_{w}=1$ for all $w \in X^{\omega}$. Thus we would like to consider the group $\left\langle a, b_{w}, c_{w}, d_{w} \mid a^{2}, b_{w}^{2}, c_{w}^{2}, d_{w}^{2}, b_{w} c_{w} d_{w}\right\rangle$. We decide to take more relations, and to set

$$
F=\left\langle a, b_{w}, c_{w}, d_{w} \mid a^{2}, b_{w}^{2}, c_{w}^{2}, d_{w}^{2}, b_{w} c_{w} d_{w}, b_{0 w}^{a}=b_{1 w}, c_{0 w}^{a}=c_{1 w}, d_{0 w}^{a}=c_{1 w}\right\rangle .
$$

The reason for this is to ensure $\bar{\psi}(\bar{\varphi}(g))=(*, g)$ for all $g \in F$. This will become clear later.

The decomposition map $\psi: \mathrm{G}_{2} \rightarrow \mathrm{G}_{2}$ ? $\operatorname{Sym}(X)$ restricts to a map $N \rightarrow$ $N^{X} \times \operatorname{Sym}(X)$, which induces a homomorphism $\bar{\psi}: F \rightarrow F$ ? $\operatorname{Sym}(X)$. We write $F$ 々 $\operatorname{Sym}(X)=F_{1}$ for short. Set $R_{0}=1 \triangleleft F$ and $R_{n+1}=\psi^{-1}\left(R_{n}^{X}\right)$ for all $n \geq 0$.

Lemma 7.3.5. $\mathrm{G}_{2}=F / \bigcup_{n \geq 0} R_{n}$.

Proof. By our choice of relations in $F$, the decomposition $\bar{\psi}$ is contracting on $F$, with nucleus $N$. Given $g \in F$ : if $g$ belongs to $R_{n}$ for some $n$, then it is clear that $g$ is trivial in $\mathrm{G}_{2}$. Conversely, if $g$ is trivial in $G$, there exists $n$ such that all $g$ 's level- $n$ states belong to $N$ and act trivially; so they are all 1 ; so $g$ belongs to $R_{n}$.

Proof of Theorem 7.3.1. Using the Reidemeister-Schreier rewriting method, we can construct a normal generating set for $R_{1}$. Indeed, $\bar{\psi}$ induces an injective map $F / R_{1} \rightarrow F_{1}$, whose image has index 4 . It is easy to write down a presentation for $F_{1}$, for example

$$
\begin{aligned}
& F_{1}=\left\langle S^{X}, \sigma\right| \sigma^{2},\left[s_{1}^{(0)}, s_{2}^{(1)}\right],\left(s^{(0)}\right)^{\sigma}=s^{(1)},\left(s^{(1)}\right)^{2}, b_{w}^{(1)} c_{w}^{(1)} d_{w}^{(1)}, \\
&\left.\left(b_{0 w}^{(1)}\right)^{a^{(1)}} b_{1 w}^{(1)},\left(c_{0 w}^{(1)}\right)^{a^{(1)}} c_{1 w}^{(1)},\left(d_{0 w}^{(1)}\right)^{a^{(1)}} d_{1 w}^{(1)}: s, s_{1}, s_{2} \in S ; w \in X^{\omega}\right\rangle
\end{aligned}
$$

with $S=\left\{a, b_{w}, c_{w}, d_{w}: w \in X^{\omega}\right\}$ and $S^{X}=\left\{s^{(x)}: s \in S, x \in X\right\}$. Here the superscript indicates the copy of $F$ inside the base group of $F_{1}$.

We choose the set $T=\left\{1, a^{(0)}, d^{(0)}, a^{(0)} d^{(0)}\right\}$ with $d^{(0)}=d_{1 w_{0}}^{(0)}$ as a transversal for $\bar{\psi}(F)$ in $F_{1}$, see Figure 7.2 . We obtain quite explicitly

$$
\begin{aligned}
& R_{1}=\left\langle\left[d_{1 w}^{a}, d_{1 w^{\prime}}^{t}\right],\left[d_{1 w}^{a},\left(c \beta^{\prime}\right)^{t}\right],\left[(c \beta)^{a},\left(c \beta^{\prime}\right)^{t}\right], b_{0 w}^{a} b_{1 w}, c_{0 w}^{a} c_{1 w}, d_{0 w}^{a} d_{1 w},\right. \\
& \left.c^{a} b_{10 w} \bar{c}^{a} b_{11 w}, \bar{c}^{a} b_{10 w} c^{a} b_{11 w}, c^{a} c_{10 w} \bar{c}^{a} c_{11 w}, \bar{c}^{a} c_{10 w} c^{a} c_{11 w}, d_{10 w}^{c^{a}} d_{11 w}\right\rangle^{F}
\end{aligned}
$$




\subsection{A Presentation of $\mathrm{G}_{2}$}

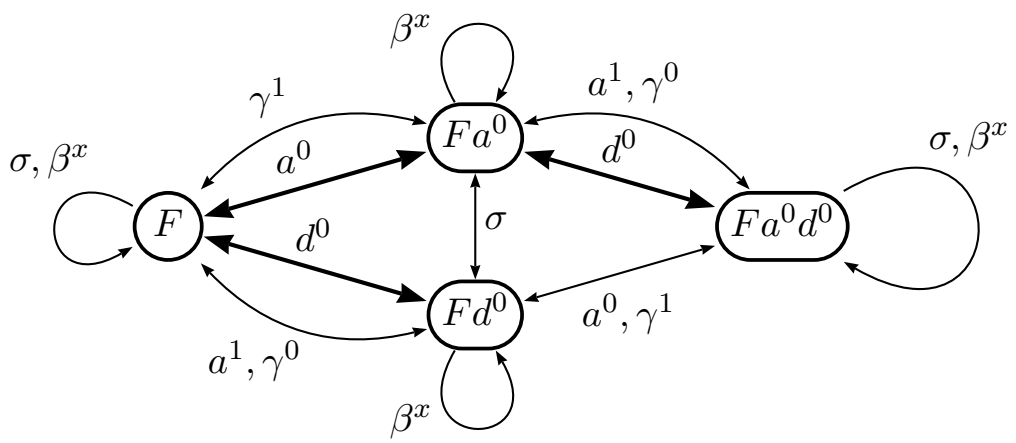

Figure 7.2: The right cosets of $F_{1} \bmod \bar{\psi}(F)$. The bold arrows correspond to the chosen Schreier system.

with $w, w^{\prime} \in X^{\omega}, c=c_{11 w_{0}}, \bar{c}=c_{10 w_{0}}, \beta, \beta^{\prime} \in\left\{b_{1 w}, c_{1 w} \mid w \in X^{\omega}\right\}$, and $t$ ranges over $\left\{1, c, c^{a}, c c^{a}\right\}$.

We now consider the homomorphism $\bar{\varphi}: F \rightarrow F$ defined by

$$
a \mapsto c^{a}, \quad b_{1 w} \mapsto d_{11 w}, \quad c_{1 w} \mapsto b_{11 w}, \quad d_{1 w} \mapsto c_{11 w} .
$$

It is clear that for all $g \in F$, we have $\bar{\psi}(\bar{\varphi}(g))=(*, g)$ for some $* \in F$. By Proposition 7.2.6, for every relation $r$ we have $\bar{\psi}(\bar{\varphi}(r))=\left(r^{\prime}, r\right)$ for some relation $r^{\prime} \in\langle a, d\rangle$. It happens that $(a d)^{4}$ is in $R_{1}$ and that $\langle a, d\rangle$ is a dihedral group of order 8 in $\mathrm{G}_{2}$. Therefore $r^{\prime}$ is in $R_{1}$ and $R_{n}$ is normally generated by $\bigcup_{i<n} \bar{\varphi}^{i}\left(R_{1}\right)$ for all $n>0$. Also, $\tilde{\varphi}(r)$ is clearly a consequence for $r$ in $\left\{a^{2}, b_{w}^{2}, c_{w}^{2}, d_{w}^{2}, b_{w} c_{w} d_{w}, b_{0 w}^{a} b_{1 w}, c_{0 w}^{a} c_{1 w}, d_{0 w}^{a} c_{1 w}\right\}$. Thus we proved the claim. 


\section{Appendix A}

\section{Examples}

We present eight examples of groups. Each of them is generated by a finite automaton, and is therefore finitely generated and self-similar. For each group $G$, we give information about the ideal $\mathcal{I}(G)$ or $\mathcal{I}_{p}(G)$.

Other properties of these groups are the following. Proofs are either very easy, or can be found in the references given for each particular group.

- The groups $\mathrm{G}, \mathfrak{G}, \ddot{\Gamma}, \Gamma, B, A, G_{\mathrm{BSV}}, \mathrm{H}$ are all self-similar, level-transitive and recurrent.

- The groups $\mathrm{G}, \mathfrak{G}, \ddot{\Gamma}, \Gamma, B, \mathrm{H}$ are regular branch. The group $G_{\mathrm{BSV}}$ is only weakly regular branch.

- The groups $\mathrm{G}, \ddot{\Gamma}, \Gamma, B$ have the congruence subgroup property, whereas the groups $\mathfrak{G}$ and $\mathrm{H}$ do not.

- The groups $\mathrm{G}, \mathfrak{G}, \ddot{\Gamma}, B$ are torsion, and the groups $A$ and $G_{\mathrm{BSV}}$ are torsionfree.

- All these examples are amenable, by [AAV09].

\section{A.1 The Grigorchuk Group}

The "first Grigorchuk group" was introduced by Grigorchuk in [Gri80] as a new Burnside group (see also [Ale72]). When Grigorchuk announced [Gri83] that the group has intermediate growth, it definitely attracted people's attention to groups acting on rooted trees.

Nowadays it remains a fundamental example, because so much is known about it. A very good introduction to this group can be found in the last chapter of [H00]. A more recent survey is [Gri05].

\section{Generators}

The Grigorchuk group is $\mathrm{G}=\langle a, b, c, d\rangle$, with

$$
a=\sigma, \quad b=(a, c), \quad c=(a, d), \quad d=(1, b) .
$$

The corresponding automaton is displayed on Figure A.1. 

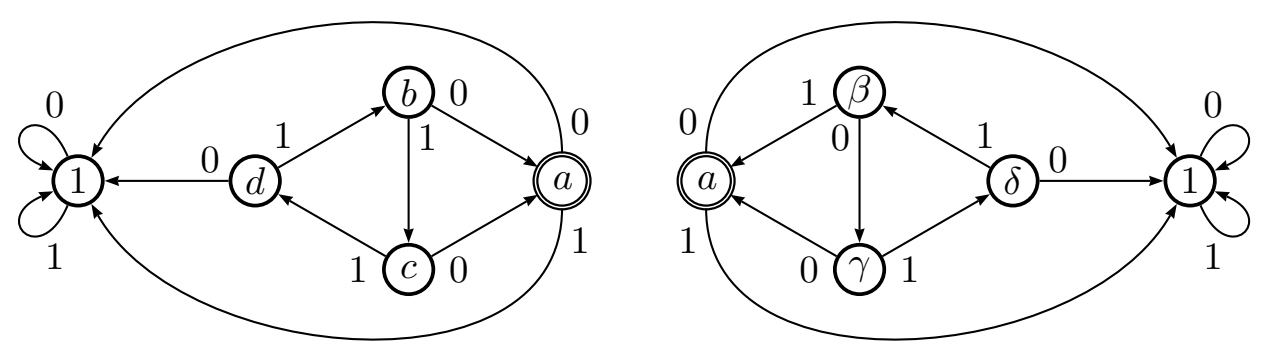

Figure A.1: The automata generating $\mathrm{G}$ (left) and $\mathfrak{G}$ (right).

\section{Equations}

Define

$$
\begin{aligned}
P_{\Delta} & =\left[\Delta^{1}+\Delta^{2}+\Delta^{3}\right], \\
P_{0} & =\left[\underline{0}+\underline{1} *\left(\Delta^{1}+\Delta^{2}\right)\right], \\
Q & =[\underline{0}] \cdot[\underline{1}]+\left[\Delta^{1} * \underline{1}+\Delta^{1} * \underline{0} * \Delta^{1}\right] .
\end{aligned}
$$

Proposition A.1.1. The ideal $\mathcal{I}(\mathrm{G})$ is generated by $\left\{P_{\Delta}, P_{0}, Q\right\}$ as a branching ideal.

Proof. Consider the ideals $I_{1}=\left(P_{\Delta}\right), I_{2}=\left(P_{\Delta}, P_{0}\right)$ and $I_{3}=\left(P_{\Delta}, P_{0}, Q\right)$. It is straightforward to check that $P_{\Delta}, P_{0}$ and $Q$ vanish on 1 .

We use Proposition 2.2.3 thrice to get the inclusion $I_{3} \subseteq \mathcal{I}(\mathrm{G})$. For this we use Equation (2.2.1) to compute the action of $S=\{a, b, c, d\}$ on $I_{d}$. We have

$$
P_{\Delta}^{g}=P_{\Delta}+P_{\Delta}(g)
$$

for all $g \in$ Aut $X^{*}$. In particular $P_{\Delta}^{s}=P_{\Delta}$ holds for all $s \in S$. We therefore have $I_{1} \subseteq \mathcal{I}(\mathrm{G})$. Further, we compute $P_{0}^{s}=P_{0}$ for $s \in\{b, c, d\}$ and $P_{0}^{a}-P_{0}=P_{\Delta}$. Therefore the inclusion $I_{2} \subseteq \mathcal{I}(\mathrm{G})$ holds. Finally, we have $Q^{s}=Q$ for $s \in\{a, d\}$ and $Q^{s}-Q=P_{0}$ for $s \in\{b, c\}$, hence $I_{3} \subseteq \mathcal{I}(\mathrm{G})$.

A direct computation shows that $\pi_{4}(\mathrm{G})$ has index $2^{3}$ in Aut $X^{4}$. Therefore $I_{3} \cap \mathcal{P}_{4}$ coincides with $\mathcal{I}(\mathrm{G}) \cap \mathcal{P}_{4}$. We use [Gri00, Proposition 9] which says in particular that $G$ is regular branch over a subgroup $K$ which contains $\operatorname{Stab}_{G}(3)$. By Corollary 2.2.8, the result follows.

Remark A.1.2. The description from Proposition A.1.1 of the elements of $\bar{G}$ coincides with the one given in [AHKŠ07, Appendix], as one can check directly. In particular, this gives the list of "forbidden patterns" of $\bar{G}$, in the sense of [Gri05, Section 7].

\section{A.2 The Twisted Twin of the Grigorchuk Group}

This group was introduced in [BS09] as a variation of the Grigorchuk group, which fails to have the congruence subgroup property. Interestingly, both groups have the same closure in Aut $X^{*}$. 


\section{Generators}

The twisted twin of the Grigorchuk group is $\mathfrak{G}=\langle a, \beta, \gamma, \delta\rangle$, with

$$
a=\sigma, \quad \beta=(\gamma, a), \quad c=(a, \delta), \quad \delta=(1, \beta) .
$$

The automaton is shown on Figure A.1.

\section{Equations}

The group $\mathfrak{G}$ has the same closure as $G$, and thus satisfies exactly the same equations.

Proposition A.2.1. The ideal $\mathcal{I}(\mathfrak{G})$ is is the same as $\mathcal{I}(\mathrm{G})$.

Proof. One could imitate the proof of Proposition A.1.1. We choose another way instead.

Using Proposition A.1.1, it is easy to see that $\mathfrak{G}$ is a subgroup of $\bar{G}$. Now $\bar{G}$ is at most 3-generated since $G$ is dense in it. This implies that the Frattini quotient of $\bar{G}$ has at most dimension 3. In fact it has dimension 3 , because it maps onto the Frattini quotient of $\pi_{3}(\mathrm{G})$, which has dimension 3. The dual of this space is generated by $[\varnothing],[\Delta]$ and $\left[\Delta^{2}\right]$. From this it is easy to check that $\mathfrak{G}$ generates the Frattini quotient of $\bar{G}$, and is thus dense in $\bar{G}$.

\section{A.3 The Gupta-Sidki Group}

Gupta and Sidki introduced in [GS83a] a series of Burnside groups for each prime $p \geq 3$. These groups are similar in flavour to the Grigorchuk group. For the first time the authors described the groups using their action on a rooted tree. What we call "the" Gupta-Sidki group is the case $p=3$.

\section{Generators}

The Gupta-Sidki group is $\ddot{\Gamma}=\langle a, t\rangle$, with

$$
a=\sigma, \quad t=\left(a, a^{-1}, t\right) .
$$

Figure A.2 shows the corresponding automaton.

\section{Equations}

Set $p=3$ and define

$$
f_{\Delta}=[\Delta], \quad f_{1}=\left[-\Delta_{1}+\Delta_{21}\right], \quad f_{2}=\left[-\underline{0}+\Delta_{11}\right] .
$$

Proposition A.3.1. The ideal $\mathcal{I}_{3}(\ddot{\Gamma})$ is generated by $\left\{f_{\Delta}, f_{1}, f_{2}\right\}$ as a branching ideal. 

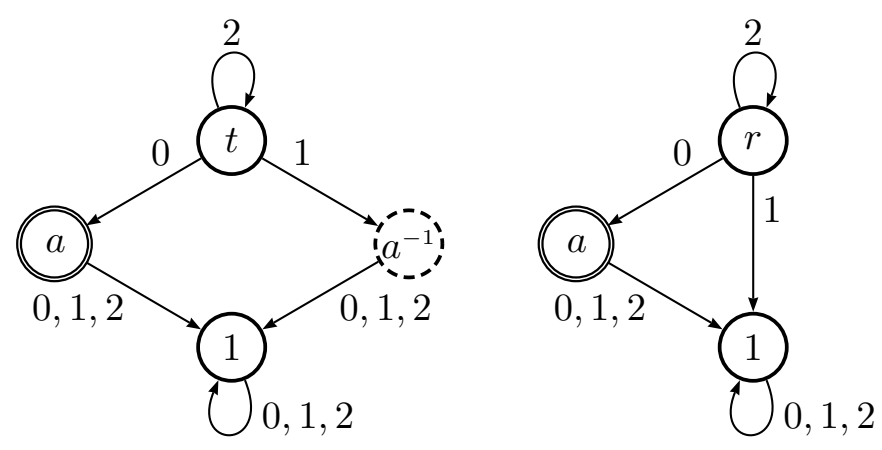

Figure A.2: The automata generating $\ddot{\Gamma}$ (left) and $\Gamma$ (right).

Proof. $f_{\Delta}$ can be viewed as a group homomorphism Aut $X^{*} \rightarrow \mathbb{F}_{3}$. It is clear that the generators of $\ddot{\Gamma}$ lie in the kernel of this map, hence so does the whole of $\ddot{\Gamma}$. We thus have $f_{\Delta} \in \mathcal{I}_{3}(\ddot{\Gamma})$.

Since $\ddot{\Gamma}$ is self-similar, $\mathcal{I}_{3}(\ddot{\Gamma})$ is branching, and we deduce that $x * f_{\Delta}$ is also in $\mathcal{I}_{3}(\ddot{\Gamma})$ for all $x \in X$. We now compute the action of $S=\{a, t\}$ on $f_{1}$ and $f_{2}$. We have

$$
\begin{array}{ll}
f_{1}^{a}-f_{1}=f_{\Delta}, & f_{1}^{t}-f_{1}=-\underline{0} * f_{\Delta}+\underline{1} * f_{\Delta} \\
f_{2}^{a}-f_{2}=f_{\Delta}-f_{1}, & f_{2}^{t}-f_{2}=\underline{1} * f_{\Delta} .
\end{array}
$$

Using Proposition 2.2.3, we deduce from the first line that $f_{1}$ is in $\mathcal{I}_{3}(\ddot{\Gamma})$. Then the second line implies $f_{2} \in \mathcal{I}_{3}(\ddot{\Gamma})$. One can check that this is enough for depth 3. Indeed, the index of $\pi_{3}(\ddot{\Gamma})$ in $\mathrm{Aut}_{3} X^{3}$ is $2^{6}$. There are four equations given by $f_{\Delta}$ and $x * f_{\Delta}$, and two which are $f_{1}$ and $f_{2}$.

We use [BG02, Proposition 8.2] which implies that $\ddot{\Gamma}$ is regular branch over $\ddot{\Gamma}^{\prime}$, and that the latter contains $\operatorname{Stab}_{\ddot{\Gamma}}(2)$. Corollary 2.2 .8 yields the result.

\section{A.4 The Fabrykowski-Gupta Group}

This group was announced [FG85,FG91] as a new example of a finitely generated group of intermediate growth.

\section{Generators}

The Fabrykowski-Gupta group is $\Gamma=\langle a, r\rangle$, with

$$
a=\sigma, \quad r=(a, 1, r) .
$$

The automaton is displayed on Figure A.2.

\section{Equations}

Set $p=3$ and define

$$
f_{1}=\left[-\Delta_{2}+\Delta_{22}\right], \quad f_{2}=\left[\Delta_{2}-\Delta_{1}+\Delta_{12}\right], \quad f_{3}=\left[-\Delta_{2}+\Delta_{1}-\Delta_{0}+\Delta_{02}\right] .
$$


Proposition A.4.1. The ideal $\mathcal{I}_{3}(\Gamma)$ is generated by $\left\{f_{1}, f_{2}, f_{3}\right\}$ as a branching ideal.

Proof. $f_{1}$ can be viewed as a group homomorphism Aut $X^{*} \rightarrow \mathbb{F}_{3}$. It is clear that the generators of $\Gamma$ lie in the kernel of this map, hence so does the whole of $\Gamma$. We thus have $f_{1} \in \mathcal{I}_{3}(\Gamma)$.

We now compute the action of $S=\{a, r\}$ on $f_{2}$ and $f_{3}$. We have

$$
\begin{array}{ll}
f_{2}^{a}-f_{2}=-f_{1}, & f_{2}^{r}-f_{2}=0, \\
f_{3}^{a}-f_{3}=f_{1}-f_{2}, & f_{3}^{r}-f_{3}=0 .
\end{array}
$$

Using Proposition 2.2.3, we deduce from the first line that $f_{2}$ is in $\mathcal{I}_{3}(\Gamma)$. Then the second line implies $f_{3} \in \mathcal{I}_{3}(\Gamma)$. One can check that this is enough for depth 3. Indeed, the index of $\pi_{3}(\Gamma)$ in $\operatorname{Aut}_{3} X^{3}$ is $2^{3}$.

We use [BG02, Proposition 6.1] which implies that $\Gamma$ is regular branch over $\Gamma^{\prime}$, and that the latter contains $\operatorname{Stab}_{\Gamma}(2)$. Therefore Corollary 2.2 .8 yields the result.

\section{A.5 Another Burnside Group}

Here is another spinal group, which we provide as an example for Theorem 2.8.8. We note that [BŠ01, Theorem 7.1] shows that the group is torsion. On the other hand, Theorem 2.8 .8 shows that the whole bounded subgroup of $\bar{B}$ is torsion. In particular, it is easy to construct torsion "twisted twins" of $B$.

Comparing Propositions A.4.1 and A.5.1, we see that $B$ can be seen as a mutant brother of $\Gamma$. The precise mutation mechanism is not clear however.

\section{Generators}

We define the group $B=\left\langle a, b_{1}, b_{2}, b_{3}, b_{4}\right\rangle$, with

$$
\begin{aligned}
a=\sigma, & b_{1}=\left(a, 1, b_{2}\right), & & b_{2}=\left(a, 1, b_{3}\right) \\
& b_{3}=\left(a^{-1}, 1, b_{4}\right), & & b_{4}=\left(1,1, b_{1}^{-1}\right) .
\end{aligned}
$$

The equalities $b_{1} b_{2}=b_{3}$ and $b_{2} b_{3}=b_{4}$ are easy to check. Thus $B$ is generated by $a, b_{1}, b_{2}$ for example.

\section{Equations}

Set $p=3$ and define

$$
\begin{array}{ll}
h_{1}=\left[-\Delta_{2}-\Delta_{22}+\Delta_{222}\right], & h_{2}=\left[\Delta_{2}-\Delta_{1}-\Delta_{12}+\Delta_{122}\right], \\
h_{3} & =\left[-\Delta_{2}+\Delta_{1}-\Delta_{0}-\Delta_{02}+\Delta_{022}\right]
\end{array}
$$

Proposition A.5.1. The ideal $\mathcal{I}_{3}(B)$ is generated by $\left\{h_{1}, h_{2}, h_{3}\right\}$ as a branching ideal. 


\section{A.5. Another Burnside Group}

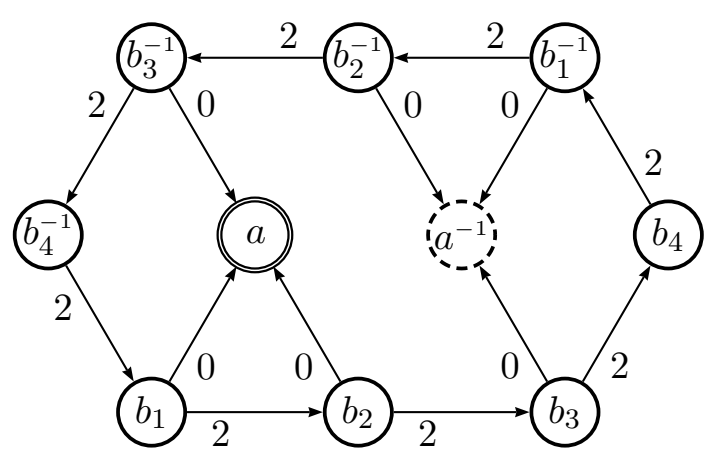

Figure A.3: The automaton generating $B$. The missing arrows connect to the trivial state, which is not displayed.

Proof. $h_{1}$ can be viewed as a group homomorphism Aut $X^{*} \rightarrow \mathbb{F}_{3}$. It is clear that the generators of $B$ lie in the kernel of this map, hence so does the whole of $B$. We thus have $h_{1} \in \mathcal{I}_{3}(B)$.

We now compute the action of $S=\left\{a, b_{1}, b_{2}\right\}$ on $h_{2}$ and $h_{3}$. We have

$$
\begin{array}{lll}
h_{2}^{a}-h_{2}=-h_{1}, & h_{2}^{b_{1}}-h_{2}=0, & h_{2}^{b_{2}}-h_{2}=0, \\
h_{3}^{a}-h_{3}=h_{1}-h_{2}, & h_{3}^{b_{1}}-h_{3}=0, & h_{3}^{b_{2}}-h_{3}=0 .
\end{array}
$$

Using Proposition 2.2.3, we deduce from the first line that $h_{2}$ is in $\mathcal{I}_{3}(B)$. Then the second line implies $h_{3} \in \mathcal{I}_{3}(B)$. One can check that this is enough for depth 4. Indeed, the index of $\pi_{4}(B)$ in $\operatorname{Aut}_{3} X^{4}$ is $2^{3}$.

By Proposition A.5.2, we may apply Corollary 2.2 .8 which yields the result.

Proposition A.5.2. The group $B$ is regular branch over $B^{\prime}$, and $B^{\prime}$ contains $\operatorname{Stab}_{B}(3)$.

Proof. The elements $b_{1}$ and $b_{2}$ commute, hence $B^{\prime}$ is generated by $\left[a, b_{1}\right]$ and $\left[a, b_{2}\right]$ as a normal subgroup. We compute

$$
\left[b_{1}, b_{4}^{-a}\right]=\underline{0} *\left[a, b_{1}\right], \quad\left[b_{1}, b_{1}^{a}\right]=\underline{0} *\left[a, b_{2}\right] .
$$

Therefore $B^{\prime}$ contains $X * B^{\prime}$. Since $B / B^{\prime}$ is generated by $a, b_{1}, b_{2}$, we have $\left[B: B^{\prime}\right] \leq 3^{3}$. On the other hand, the map $[\varnothing] \times[\Delta] \times\left[\Delta^{2}\right]$ is easily seen to be a group epimorphism from $B$ onto $\mathbb{F}_{3} \times \mathbb{F}_{3} \times \mathbb{F}_{3}$. This implies $\left[\pi_{3}(B): \pi_{3}\left(B^{\prime}\right)\right]=3^{3}$ and $\left[B: B^{\prime}\right]=3^{3}$. Therefore $B^{\prime}$ contains $\operatorname{Stab}_{B}(3)$. Since $B$ is self-similar, we have the inclusions $X^{n} * B^{\prime} \leq \operatorname{Stab}_{B}(n) \leq X^{n} * B$. The indices are finite, and therefore $X^{n} * B^{\prime}$ has finite index in $B$ for all $n \geq 0$. Thus $B$ is regular branch over $B^{\prime}$. 


\section{A.6 The Binary Adding Machine}

This is certainly the easiest example of a finitely generated, infinite, self-similar group.

\section{Generators}

We define $A=\langle\tau\rangle$, with

$$
\tau=(1, \tau) \sigma
$$

The automorphism $\tau$ is also a generator of $G_{\mathrm{BSV}}$, and can be seen on Figure A.4.

\section{Equations}

Set $p=2$ and define

$$
D_{n}=\left(\left[\underline{0}^{n}\right]+1\right)\left(\left[\underline{1}^{n}\right]+1\right), \quad R_{n}=D_{n}+1+\left[\underline{0}^{n+1}+\underline{1}^{n+1}\right]
$$

for $n \geq 0$. We set $D_{-1}=1$ to have $R_{-1}=0$. Note that $R_{0}$ is simply $[\varnothing+\Delta]$. Let $I_{n}$ be the branching ideal generated by $\left\{R_{0}, \ldots, R_{n-1}\right\}$, and set $I_{\infty}=\bigcup I_{n}$.

Proposition A.6.1. The equality $\mathcal{I}(A)=I_{\infty}$ holds.

Proof. By induction, we prove that $I_{n} \subseteq \mathcal{I}(A)$ holds for all $n \geq 0$. We have $I_{0}=(0) \subseteq \mathcal{I}(A)$, and we assume $I_{n} \subseteq \mathcal{I}(A)$ holds for some $n$. Then using Corollary A.6.4 below, together with Proposition 2.2.3, we have $R_{n} \in \mathcal{I}(A)$. Since $A$ is self-similar, $\mathcal{I}(A)$ is branching and therefore $I_{n+1} \subseteq \mathcal{I}(A)$ holds.

For the inclusion $\mathcal{I}(A) \subseteq I_{\infty}$, we note that $R_{n}$ is a nice function for all $n$. Define the sets $S_{0}=\varnothing$ and $S_{n+1}=\left(X * S_{n}\right) \cup\left\{R_{n}\right\}$ for $n \geq 0$. Then $S_{n}$ consists of nice functions, is a generating set for $I_{\infty} \cap \mathcal{P}_{n+1}$ modulo $I_{\infty} \cap \mathcal{P}_{n}$, and it is easy to see that the linear part $\operatorname{lin}\left(S_{n}\right)$ is a set of linearly independent functions. Since $S_{n}$ has $2^{n}-1$ elements, we deduce that $\pi_{n+1}\left(\operatorname{Stab}_{\mathcal{V}\left(I_{\infty}\right)}(n)\right)$ has 2 elements for all $n \geq 0$. Thus $\mathcal{V}\left(I_{\infty}\right)$ is (contained in) the closure of $A$ and we have $\mathcal{I}(A)=I_{\infty}$.

Lemma A.6.2. For all $n \geq 0$, the equalities $D_{n} \equiv\left(\underline{1} * D_{n-1}\right)([\varnothing]+1) \equiv(\underline{0} *$ $\left.D_{n-1}\right)([\varnothing]+1)$ hold modulo $I_{n}$.

Proof. It is enough to prove $D_{n} \equiv\left(\underline{1} * D_{n-1}\right)([\varnothing]+1)$. The other equality then holds by symmetry.

Modulo $R_{n-1}$ we have $\left[\underline{0}^{n}\right] \equiv\left[\underline{1}^{n}\right]+1+D_{n-1}$. Replacing this in the definition of $D_{n}$ we get $D_{n} \equiv\left(\left[\underline{1}^{n}\right]+D_{n-1}\right)\left(\left[\underline{1}^{n}\right]+1\right)$. The term $\left[\underline{1}^{n}\right]\left(\left[\underline{1}^{n}\right]+1\right)$ vanishes because $x(x+1)=x^{2}+x=0$ holds for all $x \in \mathbb{F}_{2}$. Therefore we are left with $D_{n} \equiv D_{n-1}\left(\left[\underline{1}^{n}\right]+1\right)\left(\bmod R_{n}\right)$ and induction yields

$$
D_{n} \equiv\left(\left[\underline{1}^{n}\right]+1\right)\left(\left[\underline{1}^{n-1}\right]+1\right) \cdots([\varnothing]+1) \quad\left(\bmod R_{n}\right) .
$$

The claim is now obvious.

Corollary A.6.3. For all $n \geq 0$, the equality $D_{n}^{t}-D_{n} \equiv \underline{1} * D_{n-1}$ holds modulo $I_{n}$. 
Proof. We have $D_{n}^{t} \equiv\left(\underline{0} * D_{n-1}\right)^{t}([\varnothing]+1)^{t}$. We separately compute $\left(\underline{0} * D_{n-1}\right)^{t}=$ $\underline{0}^{t} * D_{n-1}^{t @ 0}=\underline{1} * D_{n-1}$ and $[\varnothing]^{t}=[\varnothing]+1$. This yields $D_{n}^{t} \equiv\left(\underline{1} * D_{n-1}\right)[\varnothing]$, and the result follows.

Corollary A.6.4. For all $n \geq 0$, the equality $R_{n}^{t}-R_{n} \equiv 0$ holds modulo $I_{n}$.

Proof. We compute $\left(1+\left[\underline{0}^{n+1}+\underline{1}^{n+1}\right]\right)^{t}=\left[\underline{1}^{*} \underline{0}^{n}+\underline{0}^{n+1}\right]$. Using the previous corollary, we get $R_{n}^{t}-R_{n} \equiv \underline{1} * D_{n-1}+1+\left[\underline{1} * \underline{0}^{n}+\underline{1}^{n+1}\right]=\underline{1} * R_{n-1}$.

\section{Another Adding Machine}

There is only one conjugate of $\tau$ which also generates a self-similar group:

$$
\mu=\left(1, \mu^{-1}\right) \sigma
$$

This is the second generator of the group $G_{\mathrm{BSV}}$, whose generating automaton is depicted on Figure A.4.

Define the functions

$$
S_{n}=\left[\underline{0}^{n}\right] \cdot\left[\underline{1}^{n}\right]+\left[\underline{0}^{n+1}+\underline{1}^{n+1}\right],
$$

let $J_{n}$ be the branching ideal generated by $\left\{S_{0}, \ldots, S_{n-1}\right\}$ and set $J_{\infty}=\bigcup J_{n}$. Similarly to Proposition A.6.1, we have

Proposition A.6.5. The equality $\mathcal{I}(\langle\mu\rangle)=J_{\infty}$ holds.

\section{A.7 The Brunner-Sidki-Vieira Group}

This group was introduced in [BSV99] as a just nonsolvable, torsion-free group. It is generated by two adding machines.

\section{Generators}

The Brunner-Sidki-Vieira group is $G_{\mathrm{BSV}}=\langle\tau, \mu\rangle$, with

$$
\tau=(1, \tau) \sigma, \quad \mu=\left(1, \mu^{-1}\right) \sigma .
$$

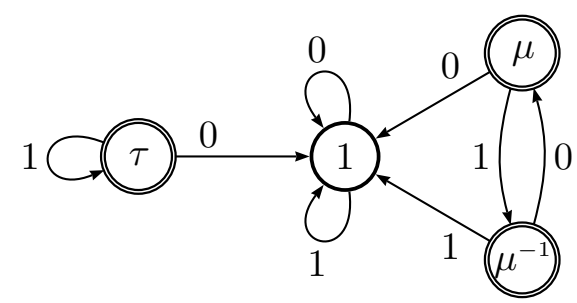

Figure A.4: The automaton generating $G_{\mathrm{BSV}}$. 


\section{Equations}

Define

$$
\begin{aligned}
& P_{0}=[\varnothing+\Delta], \\
& P_{1}=[\underline{0}] \cdot[\underline{1}]+[\underline{00}] \cdot[\underline{01}]+[\underline{10}] \cdot[\underline{11}]+\sum_{x, y \in X}[x y \bar{x}] .
\end{aligned}
$$

Let $I$ be the branching ideal generated by $P_{0}$ and $P_{1}$.

Proposition A.7.1. The ideal $\mathcal{I}\left(G_{\mathrm{BSV}}\right)$ contains $I$.

Proof. $P_{0}$ can be seen as a group epimorphism Aut $X^{*} \rightarrow \mathbb{F}_{2}$. The two generators of $G_{\mathrm{BSV}}$ lie in the kernel of $P_{0}$, therefore $P_{0}$ is in $\mathcal{I}\left(G_{\mathrm{BSV}}\right)$. Since $G_{\mathrm{BSV}}$ is self-similar, $\mathcal{I}\left(G_{\mathrm{BSV}}\right)$ contains the branching ideal $J$ generated by $P_{0}$. We then compute $P_{1}^{\tau} \equiv P_{1}$ and $P_{1}^{\mu} \equiv P_{1}$ modulo $J$, and by Proposition 2.2 .3 , this proves $P_{1}$ is in $\mathcal{I}\left(G_{\mathrm{BSV}}\right)$. Using self-similarity again yields the claim.

The group $G_{\mathrm{BSV}}$ is weakly regular branch, but not regular branch. Therefore, even as a branching ideal, $\mathcal{I}\left(G_{\mathrm{BSV}}\right)$ has no finite generating set. Luckily, there is a nice form for the infinite set of equations of the adding machine. Unfortunately, I have not found such a nice form for those of $G_{\mathrm{BSV}}$.

\section{A.8 The Hanoi Tower Group}

This group has been first considered by Grigorchuk and Sunic [GŠ06]. It is related to the "Towers of Hanoi" game in the sense that the Schreier graph of the action of $\mathrm{H}$ on $X^{n}$ is the space of moves in a 3-peg game with $n$ disks.

\section{Generators}

Consider $X=\{1,2,3\}$. The Hanoi Tower group is $\mathrm{H}=\langle a, b, c\rangle$, with

$$
a=(a, 1,1) \sigma_{23}, \quad b=(1, b, 1) \sigma_{13}, \quad c=(1,1, c) \sigma_{12} .
$$

Here $\sigma_{i j}$ is the permutation of $X$ exchanging $i$ and $j$. The automaton generating $\mathrm{H}$ is displayed on Figure A.5.

\section{Equations}

Consider $k=\mathbb{F}_{2}$ and let sign $: \operatorname{Sym}(X) \rightarrow \mathbb{F}_{2}$ be the signature homomorphism. Define sign : Aut $X^{*} \rightarrow \mathbb{F}_{2}$ as the composition of $\pi_{1}:$ Aut $X^{*} \rightarrow$ Aut $X \simeq$ $\operatorname{Sym}(X)$ with sign. We define the linear map

$$
\begin{aligned}
{[\bullet]: k\{X\} } & \rightarrow \mathcal{A}_{*} \\
v & \mapsto v * \text { sign, }
\end{aligned}
$$

and set $S=[\varnothing+\Delta]$. 
A.8. The Hanoi Tower Group

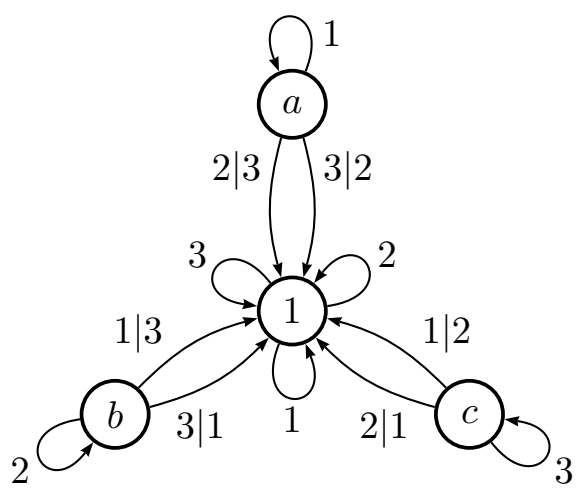

Figure A.5: The automaton generating $\mathrm{H}$.

Proposition A.8.1. The ideal $\mathcal{I}(\mathrm{H})$ is generated by $S$ as a branching ideal.

Proof. It is clear that $S$ is a group epimorphism Aut $X^{*} \rightarrow \mathbb{F}_{2}$, and that $\mathrm{H}$ lies in the kernel of this map. Since $H$ is self-similar, $\mathcal{I}(H)$ contains the branching ideal generated by $S$.

For the converse inclusion we count the elements of $\pi_{n}(\mathrm{H})$. By [BSZ09, Lemma 3.24], this finite group has the same number of elements as $\pi_{n}\left(\mathcal{V}\left(X^{*} *\right.\right.$ $S))$.

We note that $\mathrm{H}$ is regular branch. However, because its branching subgroup is not a congruence subgroup, we cannot apply Corollary 1.8.4. 


\title{
Appendix B
}

\section{Hausdorff Dimension of Some Groups Acting on the Binary Tree}

\begin{abstract}
Based on the work of Abercrombie [Abe94], Barnea and Shalev [BS97. gave an explicit formula for the Hausdorff dimension of a group acting on a rooted tree. We focus here on the binary tree $\mathcal{T}$. Abért and Virág [AV05] showed that there exist finitely generated (but not necessarily level-transitive) subgroups of Aut $\mathcal{T}$ of arbitrary dimension in $[0,1]$.

In this article we explicitly compute the Hausdorff dimension of the leveltransitive spinal groups. We then show examples of 3-generated spinal groups which have transcendental Hausdorff dimension, and exhibit a construction of 2-generated groups whose Hausdorff dimension is 1 .
\end{abstract}

\section{B.1 Introduction}

Although it is known [AV05] that finitely generated subgroups of Aut $\mathcal{T}$ may have arbitrary Hausdorff dimension, there are only very few explicit computations in the literature. Further, all known examples have rational dimension, starting with the "first Grigorchuk group" whose dimension is $5 / 8$, as we will see below. In this article we give two explicit constructions of finitely generated groups. We obtain groups of dimension 1 on one hand, and groups whose dimension is transcendental on the other hand. This is achieved by computing the dimension of the so-called spinal groups acting on the binary tree, which are generalizations of the Grigorchuk groups.

We begin by recalling the definition of Hausdorff dimension in the case of groups acting on the binary tree. In Section B.3, we define the spinal groups we are interested in. Section B.4 is devoted to the statement of Theorem B.4.4 which gives a formula for computing the Hausdorff dimension of any spinal group acting level-transitively on the binary tree; the proof is deferred to Section B.7. Finally, the construction of 3-generated spinal groups of irrational Hausdorff dimension is given in Section B.5, and groups with Hausdorff dimension 1 are exhibited in Section B.6. 


\section{B.2. Hausdorff Dimension}

\section{Acknowledgements}

I wish to thank Laurent Bartholdi who suggested these questions to me as well as many ideas for answering them.

\section{B.2 Hausdorff Dimension}

Let $\mathcal{T}$ be the infinite binary rooted tree, and let Aut $\mathcal{T}$ denote its automorphism group. It is known [BS97] that the Hausdorff dimension of a closed subgroup $G$ of Aut $\mathcal{T}$ is

$$
\operatorname{dim}_{H} G=\liminf _{m \rightarrow \infty} \frac{\log |G \bmod m|}{\log |\operatorname{Aut} \mathcal{T} \bmod m|},
$$

where the "mod $m$ " notation stands for the action on the first $m$ levels of the tree (i.e. $(G \bmod m)=G / \operatorname{Stab}[G] m$, where $\operatorname{Stab}[G] m$ is the fixator of the $m$ th level of the tree). Moreover, one easily computes $\mid$ Aut $\mathcal{T} \bmod m \mid=2^{2^{m}-1}$. This yields the more explicit formula

$$
\operatorname{dim}_{H} G=\liminf _{m \rightarrow \infty} \frac{\log _{2}|G \bmod m|}{2^{m}} .
$$

Below we will identify the vertices of $\mathcal{T}$ with the set of finite words over the alphabet $X=\{0,1\}$. We recall that there is a canonical decomposition of the elements $g \in \operatorname{Aut} \mathcal{T}$ as

$$
g=\ll g @ 0, g @ 1 \gg \sigma,
$$

with $g @ x \in \operatorname{Aut} \mathcal{T}$ and $\sigma \in \operatorname{Sym} X$. We will often identify $\operatorname{Sym} X$ with $C_{2}$, the cyclic group of order 2 , or with the additive group of $\mathbb{Z} / 2 \mathbb{Z}$, the finite field with 2 elements.

\section{B.3 Spinal Groups}

We only deal here with a specific case of the more general definition of spinal groups which can be found in [BŠ01].

Before defining the spinal group $G_{\omega}$, we need a root group $A$ which we will always take to be $A=\langle a\rangle=C_{2}$, and a level group $B$, which will be the $n$-fold direct power of $C_{2}$. We think of $B$ as an $n$-dimensional vector space over $\mathbb{Z} / 2 \mathbb{Z}$. Let $\omega=\omega_{1} \omega_{2} \ldots$ be a fixed infinite sequence of non-trivial elements from $B^{*}$, the dual space of $B$, and let $\Omega$ denote the set of such sequences. We let the non-trivial element $a$ of $A$ act on $\mathcal{T}$ by exchanging the two maximal subtrees. Next, we let each element $b \in B$ act via the recursive formulæ $b=\ll \omega_{1}(b), b @ 1 \gg$ and $b @ 1^{k}=\ll \omega_{k+1}(b), b @ 1^{k+1} \gg$. The spinal group $G_{\omega}$ is the group generated by $A \cup B$. Note that we required each $\omega_{i}$ to be non-trivial, and this implies that $G_{\omega}$ is level-transitive (i.e. $G_{\omega}$ acts transitively on $X^{n}$ for all $n \in \mathbb{N}$ ).

The syllable form of $\omega$ is $\omega_{1}^{a_{1}} \omega_{2}^{a_{2}} \ldots$ where $\omega_{i} \neq \omega_{i+1}$ and the $a_{i}$ denote multiplicities. In contrast to this we say that $\omega=\omega_{1} \omega_{2} \ldots$ is in developed form. We designate by $s_{k}$ the sum of the $k$ first terms of the sequence $\left(a_{i}\right)$, i.e. the length of the $k$-syllable prefix of $\omega$. 
Chapter B. Hausdorff Dimension of Some Groups Acting on the Binary Tree

\section{B.4 Main Theorem}

Before stating the main theorem, we need a few more definitions.

Definition B.4.1. The shift $\sigma: \Omega \rightarrow \Omega$ is defined on a sequence $\omega=\omega_{1} \omega_{2} \ldots$ by $\sigma \omega=\omega_{2} \omega_{3} \ldots$

Definition B.4.2. For a positive integer $m$ and a sequence $\omega=\omega_{1}^{a_{1}} \omega_{2}^{a_{2}} \ldots$ in syllable form, we define $s^{-1}(m)$ as the only integer that satisfies $s_{s^{-1}(m)-1}+1<$ $m \leq s_{s^{-1}(m)}+1$.

In other words, $s^{-1}(m)$ is the number of syllables of the prefix of $\omega$ of length $m-1$. If we write $\omega=\omega_{1}^{a_{1}} \omega_{2}^{a_{2}} \ldots$ in syllable form, then $\left\{\omega_{1}, \ldots, \omega_{s^{-1}(m)}\right\}$ is the set of elements of $B^{*}$ which appear in $(G \bmod m)$.

Definition B.4.3. Let $\omega=\omega_{1}^{a_{1}} \omega_{2}^{a_{2}} \ldots$ be in syllable form. Given $m \in \mathbb{N}$, we define $\operatorname{dim}(\omega \bmod m)$ as the dimension of the vector space spanned by $\omega_{1}, \ldots, \omega_{s^{-1}(m)}$. We also define $\underline{\operatorname{dim}}(\omega)$ by

$$
\underline{\operatorname{dim}}(\omega)=\liminf _{n \rightarrow \infty}\left\{\lim _{m \rightarrow \infty} \operatorname{dim}\left(\sigma^{n} \omega \bmod m\right)\right\} .
$$

The next theorem expresses the Hausdorff dimension of any level-transitive spinal group acting on the binary tree. Its proof is given in Section B.7.

Theorem B.4.4. Consider $\omega=\omega_{1}^{a_{1}} \omega_{2}^{a_{2}} \ldots$ in syllable form with $\underline{\operatorname{dim}}(\omega)=n \geq 2$. The Hausdorff dimension of $G_{\omega}$ is given by

$$
\operatorname{dim}_{H} G_{\omega}=\frac{1}{2} \liminf _{k \rightarrow \infty}\left(\frac{\Sigma_{k}}{2^{s_{k}}}+\frac{1}{2^{s_{k}}} \sum_{i=2}^{n-1} 2^{s_{\lambda_{i}}}\left(2-\frac{1}{2^{a_{\lambda_{i}}}}\right)+\frac{1}{2^{a_{k}}}\left(1-\frac{1}{2^{a_{k-1}}}\right)\right),
$$

where $\Sigma_{k}=\sum_{j=1}^{k} 2^{s_{j-1}} a_{j}$, and for each $i \in\{2, \ldots, n-1\}$ we let $\lambda_{i}(k)$ be the smallest integer such that

$$
\operatorname{dim}\left(\sigma^{s_{\lambda_{i}(k)}} \omega \bmod \left(s_{k+1}-s_{\lambda_{i}(k)}\right)\right)=i .
$$

Remark B.4.5. In case the sequence $\omega$ is eventually constant, i.e. if $\operatorname{dim}(\omega)=1$, then Proposition B.7.12 can be used to show that $\log _{2}\left|G_{\omega} \bmod m\right|$ grows linearly with $m$, whence $\operatorname{dim}_{H} G_{\omega}=0$.

In the special case where $B=C_{2} \times C_{2}$ and $\omega$ is not eventually constant, one can use the following corollary to compute $\operatorname{dim}_{H} G_{\omega}$ :

Corollary B.4.6. If $\underline{\operatorname{dim}}(\omega)=2$, then

$$
\operatorname{dim}_{H} G_{\omega}=\frac{1}{2} \liminf _{k \rightarrow \infty}\left(\frac{\Sigma_{k}}{2^{s_{k}}}+\frac{1}{2^{a_{k}}}\left(1-\frac{1}{2^{a_{k-1}}}\right)\right) .
$$

Example B.4.7. Consider $B=C_{2} \times C_{2}$. Let $\omega_{1}, \omega_{2}, \omega_{3}$ be the three non-trivial elements of $B^{*}$, and consider $\omega=\omega_{1} \omega_{2} \omega_{3} \omega_{1} \omega_{2} \omega_{3} \ldots$ The group $G_{\omega}$ is the "first Grigorchuk group", first introduced in [Gri80] (see also [Ale72]). Since the corresponding integer sequence is given by $a_{k}=1$ for all $k$, then $s_{k}=k$ and $\Sigma_{k}=2^{k}-1$. The last corollary yields

$$
\operatorname{dim}_{H} G_{\omega}=\frac{5}{8}
$$




\section{B.5. Finitely Generated Groups of Irrational Hausdorff Dimension}

Example B.4.8. The Hausdorff dimension of some spinal groups is computed in [Šun07]. Consider $B=C_{2}^{n}$, and fix a functional $\phi \in B^{*}$ and an automorphism $\rho$ (i.e. an invertible linear transformation) of $B$. We consider the sequence $\omega=\omega_{1} \omega_{2} \ldots$ defined by $\omega_{1}=\phi$ and $\omega_{n}=\rho^{*}\left(\omega_{n-1}\right)$ for $n>1$, where $\rho^{*}$ denotes the adjoint automorphism of $\rho$. We restrict attention to the case where $\rho$ and $\phi$ are such that $\underline{\operatorname{dim}}(\omega)=n$ (this is a rephrasing of the condition in [Šun07], Proposition 2). This implies that every sequence of $n$ consecutive terms of $\omega$ generates $B^{*}$. Each syllable of $\omega$ has length 1, so we can apply Theorem B.4.4 with $a_{i}=1$ and $s_{i}=i$ for all $i$, and $\lambda_{i}(k)=k-i$ for $i \in\{2, \ldots, n-1\}$ and $k \geq n$. This yields

$$
\operatorname{dim}_{H} G_{\omega}=1-\frac{3}{2^{n+1}}
$$

as was found in [Šun07].

\section{B.5 Construction of Finitely Generated Groups of Ir- rational Hausdorff Dimension}

Throughout this section we restrict to the case $\operatorname{dim}(\omega)=2$. Let $D \subset[0,1]$ be the set of possible Hausdorff dimension for $G_{\omega}$. More precisely, $D$ is defined as

$$
D=\left\{\lambda \in \mathcal{R} \mid \exists \omega \in \Omega \text { with } \underline{\operatorname{dim}}(\omega)=2 \text { and } \operatorname{dim}_{H} G_{\omega}=\lambda\right\} .
$$

Although it is not an easy thing to tell whether a given $x \in[0,1]$ lies in $D$, we are able to show the following. Let $C$ denote the Cantor set constructed by removing the second quarter of the unit interval, and iterating this process on the obtained intervals. It is easy to see that $C$ is compact and totally disconnected, and contains transcendental elements.

Theorem B.5.1. The set $D$ contains several copies of $C$, each one being the image of $C$ under an affine map with rational coefficients.

Corollary B.5.2. The set D contains transcendental elements.

Proof of Theorem B.5.1. We first define the functions

$$
\begin{aligned}
f_{a, n}: \mathcal{R} & \rightarrow \mathcal{R} \\
x & \mapsto \frac{x}{2^{a}}+\frac{a+n}{2^{a}} .
\end{aligned}
$$

We will only pay attention to the case where $a$ is a strictly positive integer and $n \in \mathbb{Z}$, and we will simply write $f_{a}$ instead of $f_{a, 0}$.

Consider $\omega=\omega_{1}^{a_{1}} \omega_{2}^{a_{2}} \ldots$ Starting from Corollary B.4.6, we can see that

$$
\begin{aligned}
\operatorname{dim}_{H} G_{\omega} & =\frac{1}{2} \liminf _{k \rightarrow \infty}\left(\frac{\Sigma_{k}}{2^{s_{k}}}+\frac{1}{2^{a_{k}}}\left(1-\frac{1}{2^{a_{k-1}}}\right)\right) \\
& =\frac{1}{2} \liminf _{k \rightarrow \infty}\left(f_{a_{k}, 1} \circ f_{a_{k-1},-1} \circ f_{a_{k-2}} \circ \cdots \circ f_{a_{1}}\right)(0) .
\end{aligned}
$$


Chapter B. Hausdorff Dimension of Some Groups Acting on the Binary Tree

Observe that the functions $f_{1}$ and $f_{2}$ define an iterated function system whose invariant set $C^{\prime}$ is the image of $C$ under the map $x \mapsto \frac{x+2}{3}$. Indeed $x_{1}=1$ and $x_{2}=\frac{2}{3}$ are the fixed points of $f_{1}$ and $f_{2}$ respectively. Write $\Delta=x_{1}-x_{2}$. Then $f_{1}\left(\left[x_{2}, x_{2}+\Delta\right]\right)=\left[x_{2}+\frac{\Delta}{2}, x_{2}+\Delta\right]$ and $f_{2}\left(\left[x_{2}, x_{2}+\Delta\right]\right)=\left[x_{2}, x_{2}+\frac{\Delta}{4}\right]$.

Now fix a point $\widehat{x} \in C^{\prime}$. There exists a sequence $\left(b_{i}\right) \in\{1,2\}^{\mathbb{N}}$ such that

$$
\widehat{x}=\lim _{k \rightarrow \infty}\left(f_{b_{0}} \circ \cdots \circ f_{b_{k}}\right)(y)
$$

for any point $y \in \mathcal{R}$. We call the sequence $\left(b_{0}, b_{1}, \ldots\right)$ the code of $\widehat{x}$. Notice that the main differences between (B.5.1) and (B.5.2) are the ordering of the factors, and the limit which is a liminf in (B.5.1).

Fix $s \in \mathbb{N}, s>2$. We define the sequence $\left(a_{i}\right) \in\{1,2, s\}^{\mathbb{N}}$ by

$$
\left(a_{1}, a_{2}, \ldots\right)=\left(b_{0}, s, b_{1}, b_{0}, s, b_{2}, b_{1}, b_{0}, s, \ldots\right)
$$

The sequence $\left(a_{i}\right)$ thus consists of prefixes of the sequence $\left(b_{i}\right)$ of increasing length, written backwards, and separated by $s$. We set $\omega=\omega_{1}^{a_{1}} \omega_{2}^{a_{2}} \omega_{1}^{a_{3}} \omega_{2}^{a_{4}} \ldots$ We will show that $\operatorname{dim}_{H} G_{\omega}$ is the image of $\widehat{x}$ under an affine map with rational coefficients. Recall that $\operatorname{dim}_{H} G_{\omega}$ is given by the liminf of

$$
\frac{1}{2}\left(f_{a_{k}, 1} \circ f_{a_{k-1},-1} \circ f_{a_{k-2}} \circ \cdots \circ f_{a_{1}}\right)(0) .
$$

Notice that the maps $f_{a, n}$ are order-preserving, and observe that $\left(f_{\alpha, 1} \circ f_{\beta,-1}\right)(0) \geq$ $\frac{3}{4}$ if $\alpha \in\{1,2\}$ and $b \geq 1$, while $\left(f_{\alpha, 1} \circ f_{\beta,-1}\right)(1)<\frac{3}{4}$ if $\alpha=s$ and $\beta \geq 1$. Therefore the lowest values in (B.5.3) are attained when $a_{k}=s$. We conclude that

$$
\operatorname{dim}_{H} G_{\omega}=\frac{1}{2} \lim _{k \rightarrow \infty}\left(f_{s, 1} \circ f_{b_{0,-1}} \circ f_{b_{1}} \circ \cdots \circ f_{b_{k}}\right)(0)=\frac{1}{2}\left(f_{s, 1} \circ f_{b_{0},-1} \circ f_{b_{0}}^{-1}\right)(\widehat{x}) .
$$

It should be noted that the maps $f_{s, 1} \circ f_{b_{0},-1} \circ f_{b_{0}}^{-1}$ do not have disjoint images. Nevertheless, it can be checked that

$$
f_{1,-1} \circ f_{1}=f_{2,-1} .
$$

This implies that a point $x \in C^{\prime}$ whose code is $2 b_{1} b_{2} \ldots$ is mapped to the same point as the point whose code is $11 b_{1} b_{2} \ldots$ On the other hand, the set of points whose code starts with a 1 is just $f_{1}\left(C^{\prime}\right)$. Under the maps $f_{s, 1} \circ f_{b_{0},-1} \circ f_{b_{0}}^{-1}$, the set $f_{1}\left(C^{\prime}\right)$ is sent to $\left(f_{s, 1} \circ f_{1,-1}\right)\left(C^{\prime}\right)$, and the maps $f_{s, 1} \circ f_{1,-1}$ are affine with rational coefficients and have disjoint images for all $s>2$. Thus $D$ contains a countable infinity of disjoint copies of $C^{\prime}$.

\section{B.6 Construction of Full-Dimensional Finitely Gen- erated Groups}

We begin with a few easy statements which will be useful.

Proposition B.6.1. Let $H \leq G$ be subgroups of Aut $X^{*}$. Then $\operatorname{dim}_{H} H \leq$ $\operatorname{dim}_{H} G$. Moreover, $\operatorname{dim}_{H} H=\operatorname{dim}_{H} G$ if the index of $H$ in $G$ is finite. 
Proof. $H \leq G$ implies $|H \bmod n| \leq|G \bmod n|$ for all $n \geq 0$. Thus $\operatorname{dim}_{H} H \leq$ $\operatorname{dim}_{H} G$. Moreover, if $k=|G: H|$ is finite then $k|H \bmod n| \geq|G \bmod n|$ for all $n \geq 0$. This yields the second claim.

Definition B.6.2. Let $H$ and $G$ be subgroups of Aut $X^{*}$ and $n$ be a positive integer. We write $H^{2^{n}} \preceq G$ if $G$ contains $2^{n}$ copies of $H$ acting on the $2^{n}$ subtrees of level $n$.

Proposition B.6.3. Let $H$ and $G$ be subgroups of Aut $X^{*}$, such that $H^{2^{n}} \preceq G$. Then $\operatorname{dim}_{H} H \leq \operatorname{dim}_{H} G$.

Proof. It is straightforward that $H^{2^{n}} \preceq G \Longrightarrow|H \bmod (m-n)|^{2^{n}} \leq|G \bmod m|$ for $m \geq n$. The conclusion follows.

We now turn to the construction of a full-dimensional group. Let $a_{1}=\sigma \in$ Aut $X^{*}$ be the permutation exchanging the two maximal subtrees and let $a_{n}$ be defined recursively as

$$
a_{n}=\ll 1, a_{n-1} \gg \sigma
$$

It is straightforward that $a_{n}$ is of order $2^{n}$. It can be viewed as a finite-depth version of the familiar adding machine $t=\ll 1, t \gg \sigma$. The important thing is that $a_{n}$ acts as a full cycle on the $n$-th level vertices of the tree, and trivially below the $n$-th level.

Next, for any element $g \in$ Aut $X^{*}$ and any word $w \in X^{*}$, we define $w *$ $g \in$ Aut $X^{*}$ as the element which acts as $g$ on the subtree $w X^{*}$, and trivially everywhere else. The following identity can be checked directly:

$$
(w * g)^{h}=w^{h} * g^{(h @ w)} .
$$

Let $g_{1}, \ldots, g_{n}$ be any elements in Aut $X^{*}$. We define the element

$$
\delta\left(g_{1}, \ldots, g_{n}\right)=\prod_{i=0}^{n-1}\left(1^{i} 0^{n-i}\right) * g_{i+1} .
$$

Notice that $1^{i} 0^{n-i}=\left(1^{n}\right)^{\left(a_{n}\right)^{2^{i}}}$, and that the product above can be taken in any order as the elements all commute (they act on different subtrees).

Lemma B.6.4. Let $G=\left\langle g_{1}, \ldots, g_{n}\right\rangle$ and $H=\left\langle a_{n}, \delta\left(g_{1}, \ldots, g_{n}\right)\right\rangle$. Then $\left(G^{\prime}\right)^{2^{n}} \preceq$ $H^{\prime}$.

Proof. The following equalities are immediate consequences of the definitions:

$$
\begin{aligned}
\delta\left(g_{1}, \ldots, g_{n}\right)^{\left(a_{n}\right)^{k}} & =\prod_{i=0}^{n-1}\left(1^{n}\right)^{\left(a_{n}\right)^{2^{i}+k}} * g_{i+1}, \\
{\left[\delta\left(g_{1}, \ldots, g_{n}\right), a_{n}^{k}\right]\left[\delta\left(g_{1}, \ldots, g_{n}\right)^{-1}, a_{n}^{k}\right] } & =\left(1^{n}\right)^{\left(a_{n}\right)^{2^{i}}} *\left[g_{j+1}, g_{i+1}\right]^{g_{j+1}^{-1}} .
\end{aligned}
$$


The second relation holds whenever there are integers $i, j \in\{0, \ldots, n-1\}$ such that $2^{i}-2^{j}=k$. These two equalities imply

$$
w *\left[g_{i}, g_{j}\right] \in H^{\prime}
$$

for all $w \in X^{n}$ and $i, j \in\{1, \ldots, n\}$. This in turn implies

$$
\left(G^{\prime}\right)^{2^{n}} \preceq H^{\prime} .
$$

Let $B_{n}=C_{2}^{n}$ be the direct product of $n$ copies of $C_{2}$. Let $\omega_{1}, \ldots, \omega_{n}$ be a basis of $B_{n}^{*}$ and consider the spinal group $G_{n}$ defined through the sequence $\omega_{1} \ldots \omega_{n} \omega_{1} \ldots \omega_{n} \ldots$ In other words $G_{n}=\left\langle a, b_{(1, n)}, \ldots, b_{(n, n)}\right\rangle$, with

$$
\begin{aligned}
& a=a_{1}=\sigma, \\
& b_{(i, n)}=\ll 1, b_{(i+1, n)} \gg \quad \text { for } i=1, \ldots, n-1, \\
& b_{(n, n)}=\ll a, b_{(1, n)} \gg \text {. }
\end{aligned}
$$

It follows from Corollary B.4.6 that $\operatorname{dim}_{H} G_{n}=1-\frac{3}{2^{n+1}}$. Define the elements

$$
\tilde{b}_{n}=\delta\left(a, b_{(1, n-3)}, \ldots, b_{(n-3, n-3)}, a_{n+1}, \tilde{b}_{n+1}\right)
$$

for $n \geq 3$, and write $H_{n}=\left\langle a_{n}, \tilde{b}_{n}\right\rangle$.

Theorem B.6.5. $H_{n}$ has Hausdorff dimension equal to 1 , for all $n \geq 3$.

Proof. Lemma B.6.4 yields

$$
\left(H_{m+1}^{\prime}\right)^{2^{m}} \preceq H_{m}^{\prime}, \quad\left(G_{m-3}^{\prime}\right)^{2^{m}} \preceq H_{m}^{\prime}
$$

for all $m \geq 3$. Thus $\operatorname{dim}_{H} H_{m}^{\prime} \geq \operatorname{dim}_{H} G_{m-3}^{\prime}$ by Proposition B.6.3, and $\operatorname{dim}_{H} H_{n}^{\prime} \geq$ $\operatorname{dim}_{H} H_{m}^{\prime}$ for all $3 \leq n \leq m$. Proposition B.6.1 allows us to state

$$
\operatorname{dim}_{H} H_{n} \geq \operatorname{dim}_{H} H_{n}^{\prime} \geq \operatorname{dim}_{H} G_{m}^{\prime}=\operatorname{dim}_{H} G_{m}
$$

for all $3 \leq n \leq m+3$. The last equality holds because $G_{m}$ is generated by $m$ elements of order 2 , so $\left|G_{m}: G_{m}^{\prime}\right|$ is finite. This yields $\operatorname{dim}_{H} H_{n}=1$ for all $n \geq 3$, since $\operatorname{dim}_{H} G_{m}=1-\frac{3}{2^{m+1}}$.

Remark B.6.6. We could easily extend this construction by taking any sequence of finitely generated groups $G_{n} \operatorname{such}$ that $\lim \sup \left(\operatorname{dim}_{H} G_{n}^{\prime}\right)=1$.

\section{B.7 Proof of the Main Theorem}

The remainder of the article is devoted to the proof of Theorem B.4.4. Our first goal is to find a recursive formula for $\left|G_{\omega} \bmod m\right|$. We begin with a few simple but very useful lemmata.

Let $\pi:$ Aut $\mathcal{T} \rightarrow($ Aut $\mathcal{T} \bmod 1) \simeq C_{2}$ be the natural epimorphism. 
Lemma B.7.1 (Folklore). The map $\phi_{n}:$ Aut $\mathcal{T} \rightarrow C_{2}$ given by $g \mapsto \prod_{w \in X^{n}} \pi(g @ w)$ is an epimorphism for all $n \in \mathbb{N}$.

For a group $G \leq \operatorname{Aut} \mathcal{T}$, we let $\operatorname{Stab}[G] n$ be the subgroup of $G$ consisting of the elements that fix the first $n$ levels of the tree. When $G=\operatorname{Aut} \mathcal{T}$ we simply write $\operatorname{Stab} n$. For $v \in X^{m}$, we define $v \phi_{n}: \operatorname{Stab} m \rightarrow C_{2}$ by $v \phi_{n}(g)=$ $\prod_{w \in X^{n}} \pi(g @(v w))$.

Corollary B.7.2. The map $v \phi_{n}:$ Stab $m \rightarrow C_{2}$ is an epimorphism for all $v \in$ $X^{m}$ and $m, n \in \mathbb{N}$.

Proof. This is straightforward because Stab $m \simeq(\operatorname{Aut} \mathcal{T})^{X^{m}}$.

In the following we let $\langle\omega \bmod m\rangle$ (resp. $\langle\omega\rangle)$ designate the vector space spanned by $\omega_{1}, \ldots, \omega_{s^{-1}(m)}$ (resp. $\left.\omega_{1}, \omega_{2}, \ldots\right)$.

Consider $\psi \in\langle\omega\rangle$ and $x \in X$. We define the homomorphisms $\bar{\psi}^{x}: \operatorname{Stab}\left[G_{\omega}\right] 1 \rightarrow$ $C_{2}$ as follows. Write $\psi=\omega_{i_{1}}+\cdots+\omega_{i_{k}}$ where the $\omega_{i_{j}}$ are pairwise distinct and all appear at least once in $\omega$. Let $\left(n_{j}+1\right)$ be the position of the first occurrence of $\omega_{i_{j}}$ in $\omega$. If $n_{j}>0$ for all $j$ then we define $\bar{\psi}^{x}=\sum_{j=1}^{k} x \phi_{n_{j}}$ (we implicitly identify $C_{2}$ with $\mathbb{Z} / 2 \mathbb{Z}$ ). Otherwise if $n_{1}=0$ we set $\bar{\psi}^{x}=\bar{x} \phi_{0}+\sum_{j=2}^{k} x \phi_{n_{j}}$, with $\bar{x}=1-x$.

By construction, $\bar{\psi}^{x}$ is a homomorphism. What may be less obvious is the following lemma (Notice that the group $\operatorname{Stab}\left[G_{\omega}\right] 1$ is generated by $\left\{b, b^{a}: b \in B\right\}$ ).

Lemma B.7.3. Consider $\psi \in\langle\omega\rangle$. Then the map $\bar{\psi}^{0}: \operatorname{Stab}\left[G_{\omega}\right] 1 \rightarrow C_{2}$ (resp. $\bar{\psi}^{1}$ ) is the homomorphism induced by $b \mapsto \psi(b)$ and $b^{a} \mapsto 1$ (resp. $b \mapsto 1$ and $\left.b^{a} \mapsto \psi(b)\right)$ for $b \in B$. In particular $\bar{\psi}^{x}$ is surjective.

Proof. This is easy to check in case $\psi$ appears in the sequence $\omega$. The general case is just a linear combination of the terms of $\omega$.

Remark B.7.4. One can define $\bar{\psi}^{x}:\left(\operatorname{Stab}\left[G_{\omega}\right] 1 \bmod m\right) \rightarrow C_{2}$ for any $\psi \in$ $\langle\omega \bmod m\rangle$ in the same way and the lemma still holds.

To proceed further we need to define some specific subgroups of $G_{\omega}$. Let $\psi$ be an element of $B^{*}$. We define the subgroup $T_{\omega}(\psi)=\langle\operatorname{ker} \psi\rangle^{G_{\omega}}$, where the superscript designates normal closure in $G_{\omega}$. It should be noted that $T_{\omega}(\psi) \leq$ $\operatorname{Stab}\left[G_{\omega}\right] 1$ for every $\psi \in B^{*}$. We will now state and prove two technical lemmata, which lead to Proposition B.7.7.

Lemma B.7.5. Let $\omega=\omega_{1}^{a_{1}} \omega_{2}^{a_{2}} \ldots$ be in syllable form; let $m \geq 1$ be an integer; let $\psi_{0}$ be a non-trivial element in $B^{*}$ such that $\psi_{0} \neq \omega_{1}$. Then

$$
\log _{2}\left|\left(G_{\omega} \bmod m\right) /\left(T_{\omega}\left(\psi_{0}\right) \bmod m\right)\right|= \begin{cases}1 & \text { if } m \leq s_{k}+1 \\ 3 & \text { if } m>s_{k}+1\end{cases}
$$

where $k$ is the greatest integer such that $\psi_{0}$ is linearly independent from $\omega_{1}, \ldots, \omega_{k}$. 
Chapter B. Hausdorff Dimension of Some Groups Acting on the Binary Tree

Proof. Case $m \leq s_{k}+1$. Let $\left\{\psi_{1}, \ldots, \psi_{\lambda}\right\}$ be a basis of $\langle\omega \bmod m\rangle$. Since $\psi_{0} \notin\langle\omega \bmod m\rangle$, the set $\left\{\psi_{0}, \ldots, \psi_{\lambda}\right\}$ is a basis of some subspace of $B^{*}$. Let $\left\{b_{0}, \ldots, b_{\lambda}\right\} \subset B$ be a dual basis, i.e. $b_{0}, \ldots, b_{\lambda}$ satisfy $\psi_{i}\left(b_{j}\right)=\delta_{i j}$ for all $i, j \in$ $\{0, \ldots, \lambda\}$.

We have $b_{1}, \ldots, b_{\lambda} \in \operatorname{ker} \psi_{0}$, so $b_{1}, \ldots, b_{\lambda} \in T_{\omega}\left(\psi_{0}\right)$. Since $\left(\operatorname{Stab}\left[G_{\omega}\right] 1 \bmod \right.$ $m$ ) is generated as a normal subgroup by the images of $b_{1}, \ldots, b_{\lambda}$, we have $\left(T_{\omega}\left(\psi_{0}\right) \bmod m\right)=\left(\operatorname{Stab}\left[G_{\omega}\right] 1 \bmod m\right)$. Therefore $\left(G_{\omega} \bmod m\right) /\left(T_{\omega}\left(\psi_{0}\right) \bmod \right.$ $m)=C_{2}$.

Case $m>s_{k}+1$. Let $\left\{\psi_{0}, \ldots, \psi_{\lambda}\right\}$ be a basis of $\langle\omega\rangle$ and let $\left\{b_{0}, \ldots, b_{\lambda}\right\}$ be a dual basis. Write $H=\operatorname{Stab}\left[G_{\omega}\right] 1=\left\langle b_{0}, \ldots, b_{\lambda}\right\rangle^{G_{\omega}}$. Then obviously $\left(G_{\omega} \bmod \right.$ $m) /(H \bmod m)=C_{2}$.

We will now prove that $(H \bmod m) /\left(T_{\omega}\left(\psi_{0}\right) \bmod m\right)=C_{2} \times C_{2}$. This group is generated by the images of $b_{0}$ and $b_{0}^{a}$. A straightforward computation shows that $b_{0}^{2}=\left[b_{0}, b_{0}^{a}\right]=1$. Therefore $(H \bmod m) /\left(T_{\omega}\left(\psi_{0}\right) \bmod m\right)$ is a quotient of $C_{2} \times C_{2}$. Consider the map $\Psi: g \mapsto\left({\overline{\psi_{0}}}^{0}(g),{\overline{\psi_{0}}}^{1}(g)\right)$. It is a surjective group homomorphism $(H \bmod m) \rightarrow C_{2} \times C_{2}$, but $\Psi\left(T_{\omega}\left(\psi_{0}\right)\right)$ is trivial. Therefore $\Psi\left(T_{\omega}\left(\psi_{0}\right) \bmod m\right)$ has index 4 in $\Psi(H \bmod m)$, which finishes the proof.

Lemma B.7.6 (Case $\left.\phi=\omega_{1}\right)$. Let $\omega=\omega_{1}^{a_{1}} \omega_{2}^{a_{2}} \ldots$ be in syllable form and set $m \geq 1$. Then

$$
\log _{2}\left|\left(G_{\omega} \bmod m\right) /\left(T_{\omega}\left(\omega_{1}\right) \bmod m\right)\right|= \begin{cases}1 & \text { if } m=1, \\ m+1 & \text { if } 1<m \leq a_{1}+1, \\ a_{1}+2 & \text { if } a_{1}+1<m \leq s_{k}+1, \\ a_{1}+3 & \text { if } s_{k}+1<m,\end{cases}
$$

where $k$ is the greatest integer such that $\omega_{1}$ is linearly independent from $\omega_{2}, \ldots, \omega_{k}$.

Proof. The case $m=1$ is very simple because $\left(T_{\omega}\left(\omega_{1}\right) \bmod 1\right)$ is the trivial group and $\left(G_{\omega} \bmod 1\right)=C_{2}$.

Define $H=\operatorname{Stab}\left[G_{\omega}\right] a_{1}+1$. If $1<m \leq a_{1}+1$, since $T_{\omega}\left(\omega_{1}\right) \leq H$, we know that $\left(T_{\omega}\left(\omega_{1}\right) \bmod m\right)$ is trivial. It is clear that $\left(G_{\omega} \bmod m\right)$ is isomorphic to a dihedral group of order $2^{m+1}$ because $\left(G_{\omega} \bmod m\right)$ is generated by two involutions $a$ and $b_{1}$, and $a b_{1}$ has order $2^{m}$ in $\left(G_{\omega} \bmod m\right)$.

Case $a_{1}+1<m \leq s_{k}+1$. Let $\left\{\omega_{i_{1}}, \ldots, \omega_{i_{\lambda}}\right\}$ be a basis of $\langle\omega\rangle$, with $\omega_{i_{1}}=\omega_{1}$. Let $\left\{b_{1}, \ldots, b_{\lambda}\right\}$ be a dual basis. It is readily checked that the group $\left\langle a, b_{1}\right\rangle$ is dihedral of order $2^{a_{1}+3}$, and a straightforward computation shows that $a^{2}=$ $b_{1}^{2}=1$ and $\left(a b_{1}\right)^{2^{a_{1}+1}} \in \operatorname{Stab}\left[G_{\omega}\right] s_{k}+1$. Since $\left(G_{\omega} \bmod m\right) /\left(T_{\omega}\left(\omega_{1}\right) \bmod m\right)$ is generated by the images of $a$ and $b_{1}$, we conclude that this group is dihedral of order $2^{a_{1}+2}$.

Finally, if $s_{k}+1<m$, it is sufficient to prove $(H \bmod m) /\left(T_{\omega}\left(\omega_{1}\right) \bmod m\right) \simeq$ $C_{2}$. By the above we know that this group is generated by the image of $\left(a b_{1}\right)^{2^{a_{1}+1}}$, and that this element is of order 2. Hence $(H \bmod m) /\left(T_{\omega}\left(\omega_{1}\right) \bmod m\right)$ is a quotient of $C_{2}$. Express $\omega_{1}$ as a linear combination of $\omega_{2}, \ldots, \omega_{k+1}: \omega_{1}=\omega_{i_{1}}+$ $\cdots+\omega_{i_{\lambda}}$. Let $n_{j}$ be the position of the last occurrence of $\omega_{i_{j}}$ in $(\omega \bmod m)$. Fix $v=0^{a_{1}+1}$ and define $\overline{\omega_{1}}:(H \bmod m) \rightarrow C_{2}$ by $\overline{\omega_{1}}=\sum_{j=1}^{\lambda} v \phi_{n_{j}}$. Then $\overline{\omega_{1}}$ is surjective but $\overline{\omega_{1}}\left(T_{\omega}\left(\omega_{1}\right) \bmod m\right)$ is trivial. This completes the proof. 
Proposition B.7.7. Let $\omega=\omega_{1}^{a_{1}} \omega_{2}^{a_{2}} \ldots$ be in syllable form and set $m>a_{1}$. Then

$\log _{2}\left|G_{\omega} \bmod m\right|=2+a_{1}+\delta(m)+2^{a_{1}}\left(\log _{2}\left|G_{\sigma^{a_{1}}} \bmod \left(m-a_{1}\right)\right|-2 \delta(m)-1\right)$, with

$$
\delta(m)= \begin{cases}0 & \text { if } \omega_{1} \text { is linearly independent from } \omega_{2}, \ldots, \omega_{s^{-1}(m)}, \\ 1 & \text { otherwise }\end{cases}
$$

Proof. For $m>a_{1}$, Lemma B.7.6 gives

$$
\log _{2}\left|\left(G_{\omega} \bmod m\right) /\left(T_{\omega}\left(\omega_{1}\right) \bmod m\right)\right|= \begin{cases}a_{1}+2 & \text { if } m \leq s_{k}+1, \\ a_{1}+3 & \text { if } m>s_{k}+1,\end{cases}
$$

where $k$ is the greatest integer such that $\omega_{1}$ is linearly independent from $\omega_{2}, \ldots, \omega_{k}$. We can rewrite this equation as

$$
\log _{2}\left|G_{\omega} \bmod m\right|=a_{1}+2+\delta(m)+\log _{2}\left|T_{\omega}\left(\omega_{1}\right) \bmod m\right| .
$$

Next, iteration of the relation $T_{\omega}\left(\omega_{1}\right)=T_{\sigma \omega}\left(\omega_{1}\right) \times T_{\sigma \omega}\left(\omega_{1}\right)$ gives

$$
T_{\omega}\left(\omega_{1}\right)=\underbrace{T_{\sigma^{a_{1} \omega}}\left(\omega_{1}\right) \times \cdots \times T_{\sigma^{a_{1} \omega}}\left(\omega_{1}\right)}_{2^{a_{1}}}
$$

therefore

$$
\log _{2}\left|T_{\omega}\left(\omega_{1}\right) \bmod m\right|=2^{a_{1}} \log _{2}\left|T_{\sigma^{a_{1}} \omega}\left(\omega_{1}\right) \bmod \left(m-a_{1}\right)\right| .
$$

But Lemma B.7.5 yields

$$
\log _{2}\left|\left(G_{\sigma^{a_{1}} \omega} \bmod \left(m-a_{1}\right)\right) /\left(T_{\sigma^{a_{1}} \omega}\left(\omega_{1}\right) \bmod \left(m-a_{1}\right)\right)\right|= \begin{cases}1 & \text { if } m \leq s_{k^{\prime}}+1, \\ 3 & \text { if } m>s_{k^{\prime}}+1,\end{cases}
$$

where $k^{\prime}$ is the greatest integer such that $\omega_{1}$ is linearly independent from $\omega_{2}, \ldots, \omega_{k^{\prime}}$, i.e. $k=k^{\prime}$. Therefore we can rewrite the preceding equation as

$$
\log _{2}\left|G_{\sigma^{a_{1} \omega}} \bmod \left(m-a_{1}\right)\right|=1+2 \delta(m)+\log _{2}\left|T_{\sigma^{a_{1} \omega}}\left(\omega_{1}\right) \bmod \left(m-a_{1}\right)\right| .
$$

Equations (B.7.1), (B.7.2) and (B.7.3) give the result.

Now the technical part is over, and the following statements and their proof, including the proof of Theorem B.4.4, are easy consequences of what has been shown above.

Proposition B.7.8. Let $\omega=\omega_{1}^{a_{1}} \omega_{2}^{a_{2}} \ldots$ be in syllable form and consider $\lambda \in \mathbb{N}$ and $m>s_{\lambda}$. Then

$$
\begin{aligned}
\log _{2}\left|G_{\omega} \bmod m\right|=3+2^{s_{0}}(1+ & \left.a_{1}+\delta_{1}-2 \delta_{0}\right)+\cdots+2^{s_{\lambda-1}}\left(1+a_{\lambda}+\delta_{\lambda}-2 \delta_{\lambda-1}\right) \\
& +2^{s_{\lambda}}\left(\log _{2}\left|G_{\sigma^{s_{\lambda}} \omega} \bmod \left(m-s_{\lambda}\right)\right|-2 \delta_{\lambda}-1\right)
\end{aligned}
$$


Chapter B. Hausdorff Dimension of Some Groups Acting on the Binary Tree

with

$$
\delta_{j}=\delta_{j}(m)= \begin{cases}0 & \text { if } \omega_{j} \text { is linearly independent from } \omega_{j+1}, \ldots, \omega_{s^{-1}(m)}, \\ 1 & \text { otherwise. }\end{cases}
$$

(We set $\delta_{0}=1$ and $\left.s_{0}=0\right)$.

Proof. This follows directly from $\lambda$ applications of Proposition B.7.7.

Corollary B.7.9. Let $\lambda$ be such that the space spanned by $\omega_{\lambda+1}, \ldots, \omega_{s^{-1}(m)}$ contains all $\omega_{j}$ with $1 \leq j \leq s^{-1}(m)$. Then

$$
\log _{2}\left|G_{\omega} \bmod m\right|=3+\Sigma_{\lambda}+2^{s_{\lambda}}\left(\log _{2}\left|G_{\sigma^{s} \lambda \omega} \bmod \left(m-s_{\lambda}\right)\right|-3\right),
$$

with

$$
\Sigma_{\lambda}=2^{s_{0}} a_{1}+\cdots+2^{s_{\lambda-1}} a_{\lambda} .
$$

Lemma B.7.10. Let $\omega=\omega_{1}^{a_{1}} \omega_{2}^{a_{2}} \ldots$ be in syllable form. Given $m>a_{1}+1$, if $\lambda$ is the smallest integer such that $\omega_{\lambda}$ is linearly independent from $\omega_{\lambda+1}, \ldots, \omega_{s^{-1}(m)}$, then

$\log _{2}\left|G_{\omega} \bmod m\right|=3+\Sigma_{\lambda}+2^{s_{\lambda}+1}-2^{s_{\lambda-1}}+2^{s_{\lambda}}\left(\log _{2}\left|G_{\sigma^{s_{\lambda}} \omega} \bmod \left(m-s_{\lambda}\right)\right|-3\right)$.

Proof. This is just a consequence of Proposition B.7.8 and Corollary B.7.9.

Remark B.7.11. If $1<m \leq a_{1}+1$ then $\left(G_{\omega} \bmod m\right)$ is just a dihedral group of order $m+1$, whence

$$
\log _{2}\left|G_{\omega} \bmod m\right|=m+1 .
$$

We are naturally led to the following proposition.

Proposition B.7.12. Consider $\omega=\omega_{1}^{a_{1}} \omega_{2}^{a_{2}} \ldots$ in syllable form with $\operatorname{dim}(\omega \bmod$ $m)=n$. Then

$\log _{2}\left|G_{\omega} \bmod m\right|=3+\Sigma_{s^{-1}(m)-1}+\sum_{i=2}^{n-1}\left(2^{s_{\lambda_{i}}+1}-2^{s_{\lambda_{i}-1}}\right)-2^{s_{\lambda_{1}-1}}+2^{s_{\lambda_{1}}}\left(m-s_{\lambda_{1}}\right)$,

where $\lambda_{j}$ is the smallest integer such that $\operatorname{dim}\left(\sigma^{s_{\lambda_{j}}} \omega \bmod \left(m-s_{\lambda_{j}}\right)\right)=j$, for each $j \in\{1, \ldots, n-1\}$.

Proof. We simply apply the previous lemma $n-1$ times to obtain

$$
\begin{aligned}
& \log _{2}\left|G_{\omega} \bmod m\right| \\
& \quad=3+\Sigma_{\lambda_{1}}+\sum_{i=1}^{n-1}\left(2^{s_{\lambda_{i}}+1}-2^{s_{\lambda_{i}-1}}\right)+2^{s_{\lambda_{1}}}\left(\log _{2}\left|G_{\sigma^{{ } \lambda_{1} \omega}} \bmod \left(m-s_{\lambda_{1}}\right)\right|-3\right) .
\end{aligned}
$$




\section{B.7. Proof of the Main Theorem}

Next, we have $\operatorname{dim}\left(\sigma^{s_{1}} \omega \bmod \left(m-s_{\lambda_{1}}\right)\right)=1$ and $\lambda_{1}=s^{-1}(m)-1$. Remark B.7.11 yields

$$
\log _{2}\left|G_{\omega} \bmod m\right|=3+\Sigma_{s^{-1}(m)-1}+\sum_{i=1}^{n-1}\left(2^{s_{\lambda_{i}}+1}-2^{s_{\lambda_{i}-1}}\right)+2^{s_{\lambda_{1}}}\left(m-s_{\lambda_{1}}-2\right) .
$$

The result is obtained by extracting the first term of the sum.

We are now ready to prove Theorem B.4.4, which we restate here.

Theorem B.7.13. Consider $\omega=\omega_{1}^{a_{1}} \omega_{2}^{a_{2}} \ldots$ in syllable form with $\underline{\operatorname{dim}}(\omega)=n \geq$

2. The Hausdorff dimension of $G_{\omega}$ is computed as

$$
\operatorname{dim}_{H} G_{\omega}=\frac{1}{2} \liminf _{k \rightarrow \infty}\left(\frac{\Sigma_{k}}{2^{s_{k}}}+\frac{1}{2^{s_{k}}} \sum_{i=2}^{n-1} 2^{s_{\lambda_{i}}}\left(2-\frac{1}{2^{a_{\lambda_{i}}}}\right)+\frac{1}{2^{a_{k}}}\left(1-\frac{1}{2^{a_{k-1}}}\right)\right),
$$

where for each $i \in\{2, \ldots, n-1\}$ we let $\lambda_{i}(k)$ be the smallest integer such that

$$
\operatorname{dim}\left(\sigma^{s_{\lambda_{i}(k)}} \omega \bmod \left(s_{k+1}-s_{\lambda_{i}(k)}\right)\right)=i .
$$

Proof. Starting with Equation (B.7.4), we write $k=s^{-1}(m)$. Recalling $\lambda_{1}=$ $k-1$, we compute

$$
\begin{aligned}
& \frac{\log _{2}\left|G_{\omega} \bmod m\right|}{2^{m}}= \\
& \frac{1}{2^{m-s_{k}}}\left(\frac{3}{2^{s_{k}}}+\frac{\Sigma_{k-1}}{2^{s_{k}}}+\frac{1}{2^{s_{k}}} \sum_{i=2}^{n-1}\left(2^{s_{\lambda_{i}}+1}-2^{s_{\lambda_{i}-1}}\right)-\frac{1}{2^{s_{k}-s_{k-2}}}+\frac{1}{2^{a_{k}}}\left(m-s_{k-1}\right)\right) .
\end{aligned}
$$

If we fix $k$ and consider $m$ such that $s_{k-1}+1<m \leq s_{k}+1$, the $\lambda_{i}$ 's do not depend on $m$. We easily check that the expression is minimal when $m=s_{k}+1$. Therefore

$$
\operatorname{dim}_{H} G_{\omega}=\frac{1}{2} \liminf _{k \rightarrow \infty}\left(\frac{\Sigma_{k}}{2^{s_{k}}}+\frac{1}{2^{s_{k}}} \sum_{i=2}^{n-1}\left(2^{s_{\lambda_{i}}+1}-2^{s_{\lambda_{i}-1}}\right)-\frac{1}{2^{a_{k}+a_{k-1}}}+\frac{1}{2^{a_{k}}}\right) .
$$

The result follows. 


\title{
Appendix C
}

\section{The Congruence Subgroup Problem for Branch Groups ${ }^{1}$}

\begin{abstract}
We state and study the congruence subgroup problem for groups acting on rooted tree, and for branch groups in particular. The problem is reduced to the computation of the congruence kernel, which we split into two parts: the branch kernel and the rigid kernel. In the case of regular branch groups, we prove that the first one is Abelian while the second has finite exponent. We also establish some rigidity results concerning these kernels.
\end{abstract}

We work out explicitly known and new examples of non-trivial congruence kernels, describing in each case the group action. The Hanoi tower group receives particular attention due to its surprisingly rich behaviour.

\section{C.1 Introduction}

One often encounters infinite mathematical objects that are arbitrarily well approximated by finite (quotient) objects. The question then arises as to which properties of the infinite object one can read in the finite objects. As a classical example [Sel51], the equation $3 x^{3}+4 y^{3}+5 z^{3}=0$ admits non-trivial solutions in $\mathbb{Z} / n \mathbb{Z}$ for all $n$, but no non-trivial solution in $\mathbb{Z}$.

As another example, consider the integers $\mathbb{Z}$ approximated by the finite rings $\mathbb{Z} / 2^{n} \mathbb{Z}$. The integer 3 is invertible in each of these finite quotients, but not in $\mathbb{Z}$. More pedantically, the ideal $3 \mathbb{Z}$ has index 3 in $\mathbb{Z}$ but its closure in $\varliminf^{\lim } \mathbb{Z} / 2^{n} \mathbb{Z}$ has index 1.

We concentrate on group theory. We have a group $G$ acting on a set $X$; suppose this set admits $G$-equivariant finite quotients $X_{n}$, on which $G$ induces permutation groups $G_{n}$. Saying that $G$ 's action on $X$ is arbitrarily well approximated by actions on the $X_{n}$ amounts to saying that $G$ embeds in the profinite group $\bar{G}=\lim _{n} G_{n}$. On the other hand, $G$ also embeds in its profinite completion $\widehat{G}$, which maps naturally onto $\bar{G}$. The congruence problem asks to compare these

\footnotetext{
${ }^{1}$ Joint work with Laurent Bartholdi and Pavel Zalesskii
} 


\section{C.1. Introduction}

profinite groups, or more quantitatively to describe the kernel of the map $\widehat{G} \rightarrow \bar{G}$, the congruence kernel.

Let $G$ be a group acting faithfully on a locally finite rooted tree $T$. Then $G$ is residually finite since the intersection $\bigcap_{n} \operatorname{Stab}_{G}(n)$ of the pointwise stabilizers of the levels $T_{n}$ is trivial. It follows that $G$ is Hausdorff with respect to two topologies: the profinite topology and the topology defined by level stabilizers $\operatorname{Stab}_{G}(n)$. We shall call the latter congruence topology. Therefore, as in the classical congruence subgroup problem [BLS64] we can ask whether these two topologies coincide. We can follow this analogy further, completing $G$ with respect to these two topologies, and define a congruence kernel $C$ as the kernel of the natural epimorphism of the completions $\widehat{G} \longrightarrow \bar{G}$ (it is worth mentioning that $\bar{G}$ coincides with the closure of $G$ in the profinite group Aut $(T))$. Then the congruence subgroup problem can be formulated as computation of the congruence kernel.

Denote by $\operatorname{Rist}_{G}(v)$ and call the rigid stabilizer of $v$ the subgroup of $G$ consisting of the elements that move only vertices which are in the subtree rooted at $v$. Let $\operatorname{Rist}_{G}(n)$ be the group generated by $\operatorname{Rist}_{G}(v)$ for all $v$ at level $n$. We say that $G$ is a branch group if it admits an action on a rooted tree $T$ such that $\operatorname{Rist}_{G}(n)$ is of finite index for every $n$. The class of branch groups has been studied intensively during the last two decades [BGŠ03]. We also say the group $G$ is level-transitive if it acts transitively on each level $T_{n}$. Note that we make that assumption in Theorems A-F, but we do not require it as part of the definition of a branch group, so as to cover potentially interesting examples such as those in [Nek07].

If $T$ is a regular rooted tree, meaning the number of descendants of every vertex is constant, we say $G \leq \operatorname{Aut}(T)$ is self-similar if for every $g \in G$ and $v \in T$ the composition $T \rightarrow T_{v} \rightarrow T_{v^{g}} \rightarrow T$ defines an element of $G$; here $T_{v}$ denotes the subtree below $v$.

For a branch group, rigid stabilizers of levels define another profinite topology that we shall call branch topology. This topology is stronger than the congruence and weaker than the profinite topology. Denote by $\widetilde{G}$ the completion of $G$ with respect to the branch topology. Then for the branch group $G$ we have the following kernels:

Congruence kernel: $\operatorname{ker}(\widehat{G} \rightarrow \bar{G})$;

Branch kernel: $\operatorname{ker}(\widehat{G} \rightarrow \widetilde{G})$;

Rigid kernel: $\operatorname{ker}(\widetilde{G} \rightarrow \bar{G})$.

In this paper we are concerned with determining the structure of these kernels. However the preliminary question of great importance is

Question C.1.1. Do any of the kernels depend on the branch representation of $\Gamma ?$

In other words, are these kernels invariants of the group $G$, or only of its action on a tree?

The situation is quite clear in the case of self-similar, regular branch groups. A group $G$ acting faithfully on a rooted tree is regular branch if there is a subgroup 
$K$ in $G$ such that $K$ contains a copy $v * K$ of $K$ acting on the subtree rooted at $v$ for every vertex $v$ of the tree, and the subgroup generated by the $v * K$ for all $v$ of given length has finite index in $G$. For a regular branch group the congruence kernel does not depend on the representation. We shall prove the following

Theorem C - A. Let $\Gamma$ be a group and consider two level-transitive, self-similar, regular branch representations $\Gamma \rightarrow$ Aut $T_{i}$ with corresponding closures $\bar{\Gamma}_{i}$. The congruence kernel $\mathcal{K}_{1}=\operatorname{ker}\left(\widehat{\Gamma} \rightarrow \bar{\Gamma}_{1}\right)$ is equal to the congruence kernel $\mathcal{K}_{2}=$ $\operatorname{ker}\left(\widehat{\Gamma} \rightarrow \bar{\Gamma}_{2}\right)$.

This theorem uses the following structure result:

Theorem C - B. Let $\Gamma$ be a level-transitive, self-similar, regular branch group. Then the branch kernel is Abelian and the rigid kernel has finite exponent.

Summarizing the information for the congruence kernel we can state the following

Theorem C - C. Let $\Gamma$ be a level-transitive, self-similar, regular branch group. Then the congruence kernel $\operatorname{ker}(\widehat{\Gamma} \rightarrow \bar{\Gamma})$ does not depend on a branch representation and is Abelian by finite exponent.

For general branch groups (i.e. not regular branch) the situation is possibly more complicated. We have the following partial result concerning the independence of the representation:

Theorem C - D. Let $\Gamma$ be a level-transitive branch group and consider two leveltransitive branch representations $\rho_{i}: \Gamma \rightarrow$ Aut $T_{i}$ of $\Gamma$ with $i=1,2$. Write $\widetilde{\Gamma}_{i}$ and $\bar{\Gamma}_{i}$ for the corresponding branch and congruence completion and denote the branch kernels by $\mathcal{K}_{i}=\operatorname{ker}\left(\widehat{\Gamma} \rightarrow \widetilde{\Gamma}_{i}\right)$.

Then $\mathcal{K}_{i}$ is in the congruence kernel $\operatorname{ker}\left(\widehat{\Gamma} \rightarrow \bar{\Gamma}_{j}\right)$ for all $i, j \in\{1,2\}$.

That is, the branch kernel is contained in the congruence kernel of any other representation. This theorem is heavily based on the observation that the branch kernel is Abelian. Moreover, it can be considered as a profinite $\widetilde{\Gamma}$-module that is described by the following

Theorem C - E. Let $\Gamma \leq$ Aut $T$ be a level-transitive branch group. There is an Abelian group A such that the branch kernel $\mathcal{K}=\operatorname{ker}(\widehat{\Gamma} \rightarrow \widetilde{\Gamma})$ is isomorphic to $\widehat{\mathbb{Z}}[[\widetilde{\Gamma}]] \widehat{\otimes}_{\left.\widehat{\mathbb{Z}}_{[}\left[\widetilde{\Gamma}_{w}\right]\right]} A$ qua profinite $A[[\widetilde{\Gamma}]]$-module. Here $\widetilde{\Gamma}_{w}$ is the stabilizer in $\widetilde{\Gamma}$ of a point $w \in \partial T$ of the boundary of the tree, where the action of $\widetilde{\Gamma}$ on $T$ is given by the natural map $\widetilde{\Gamma} \rightarrow \bar{\Gamma} \leq$ Aut $T$.

Moreover, $A$ can be explicitly computed as

$$
\lim _{\substack{v<w \\ k \geq 0}} \operatorname{Rist}_{\Gamma}(v) /\left(\operatorname{Rist}_{\Gamma}(v)^{\prime} \operatorname{Rist}_{\Gamma}(v)^{k}\right)
$$

Corollary C - F. Let $\Gamma$ be a just-infinite, level-transitive, self-similar, regular branch group. The branch kernel $\mathcal{K}=\operatorname{ker}(\widehat{\Gamma} \rightarrow \widetilde{\Gamma})$ has finite exponent. 


\section{C.1. Introduction}

The first main examples, the Grigorchuk and Gupta-Sidki groups, were shown in [BG02] to have a trivial congruence kernel. This information was vital in obtaining a description of the top of the subgroup lattice of these groups - the lattice was computed in a finite quotient, and validated by a quantitative version of the congruence property. Pervova constructed in [Per07] the first examples of groups generated by automata without the congruence property. We revisit her examples, proving that these groups have trivial rigid kernel, and giving additional information on the branch kernel (as in particular its structure as a $\Gamma$-module). We compute the kernels for the Hanoi tower group, which is the first example where both the rigid and the branch kernel are non-trivial.

\section{C.1.1 Basic Definitions and Notation}

We introduce the necessary vocabulary describing groups acting on rooted trees. The main notions are summarized in Table C.1.

\begin{tabular}{|c|c|c|c|}
\hline symbol & \multicolumn{2}{|l|}{ name } & \\
\hline $\begin{array}{c}w * g \\
G_{\# v} \\
K \\
G_{\#} \\
X^{n} * K\end{array}$ & \multicolumn{2}{|c|}{$\begin{array}{l}w \text {-translate of } g \\
\text { branching subgroup }\end{array}$} & $\begin{array}{l}\text { projection of } g \text { on the } w \text {-th coordinate in Aut } X^{*} \rightarrow \\
\text { Aut } X^{*} \imath \text { Aut } X^{n} \\
\text { element of } \operatorname{Rist}(w) \text { with }(w * g) @ w=g \\
\text { those } g \in G \text { with } v * g \in G \\
\text { subgroup with } v * K \leq K \text { for all } v \in X^{*} \\
\text { maximal branching subgroup of } G \\
\text { product of } v * K \text { for all } v \in X^{n}\end{array}$ \\
\hline $\begin{array}{l}\operatorname{Stab}_{G}(n) \\
\operatorname{Rist}_{G}(w)\end{array}$ & \multicolumn{2}{|c|}{$\begin{array}{l}\text { stabilizer of } w \\
n \text {-th level stabilizer } \\
\text { rigid stabilizer of } w \\
n \text {-th rigid stabilizer }\end{array}$} & $\begin{array}{l}\text { subgroup of } G \text { consisting of the elements which fix } \\
w \\
\text { intersection of } \operatorname{Stab}_{G}(w) \text { with } w \text { ranging over } X^{n} \\
\text { subgroup of } G \text { consisting of the elements moving } \\
\text { only descendants of } w \\
\text { group generated by } \operatorname{Rist}_{G}(w) \text { with } w \text { ranging over } \\
X^{n}\end{array}$ \\
\hline \multicolumn{2}{|c|}{$\begin{array}{l}\text { level-transitive } \\
\text { self-similar } \\
\text { recurrent } \\
\text { weakly branch } \\
\text { branch } \\
\text { weakly regular branch } \\
\text { regular branch }\end{array}$} & \multicolumn{2}{|c|}{$\begin{array}{l}\text { the action of } G \text { is transitive on each level } \\
\text { the image of } G \rightarrow \operatorname{Aut} X^{*} \imath \operatorname{Sym}(X) \text { is contained in } G \imath \operatorname{Sym}(X \\
\operatorname{Stab}_{G}(v) @ v=G \text { for all } v \in X^{*} \\
\operatorname{Rist}_{G}(v) \neq 1 \text { for all } v \in X^{*} \\
\operatorname{Rist}_{G}(n) \text { has finite index in } G \text { for all } n \geq 0 \\
G \text { contains a non-trivial branching subgroup } \\
\text { weakly regular branch, and } X^{n} * K \text { has finite index in } G \text { for } \\
\text { all } n \geq 0\end{array}$} \\
\hline
\end{tabular}

Table C.1: Symbols, subgroups, and main properties of groups acting on rooted trees. 


\section{Rooted Trees}

A rooted tree is a directed graph which is connected, and such that there is a unique vertex with no incoming edge (the root) and all the other vertices have exactly one incoming edge. The level of a vertex $v$ is the combinatorial distance between the root and $v$. A rooted tree is locally finite if every vertex has finite valency. The boundary of a rooted tree $T$ is the set $\partial T$ consisting of infinite directed paths starting from the root. It is a Cantor set if $T$ is locally finite but has infinite boundary.

A locally finite rooted tree $T$ is spherically homogeneous if there are positive integers $d_{i}$ such that every vertex of level $i$ has exactly $d_{i}$ outgoing edges. The sequence $d_{i}$ is the signature of $T$. A regular rooted tree is a spherically homogeneous tree whose signature is a constant sequence. A $d$-ary rooted tree is a regular rooted tree with signature $d_{i}=d$. We shall identify the vertices of a $d$-ary rooted tree with the elements of the free monoid $X^{*}$ with basis $X$, where $X$ is a set with $d$ elements. We shall use the notation $X^{*}$ for regular rooted trees, and use $T$ to denote arbitrary spherically homogeneous rooted trees. In this notation, the root corresponds to the identity of $X^{*}$, and we shall let $X^{n}$ designate the set of vertices of level $n$. We shall often refer to words of length $n$ to designate the elements of $X^{n}$, i.e. the vertices of level $n$. The boundary of $X^{*}$ is $X^{\omega}$, the Cantor set of (right) infinite sequences over the alphabet $X$.

Consider two vertices $u, v$ of a rooted tree $T$. We say $u$ is a prefix of $v$ (or $v$ is a descendant of $u$ ) if there is a directed path going from $u$ to $v$. In such a situation we write $u \leq v$. The set of descendants of $u$ form the subtree rooted at $u$.

\section{Automorphisms of Rooted Trees}

An automorphism of a rooted tree is a directed graph automorphism, i.e. a permutation of the vertices which preserves the edges and their orientation. It is easy to see that an automorphism preserves the level of every vertex.

Let $T$ be a rooted tree and consider a vertex $v$ of $T$. The rigid stabilizer of $v$ is the subgroup of Aut $T$ consisting of the elements that move only vertices which are in the subtree rooted at $v$. We denote this subgroup by $\operatorname{Rist}(v)$. If $T$ is spherically homogeneous then $\operatorname{Rist}(u)$ is isomorphic to $\operatorname{Rist}(v)$ when $u$ and $v$ are on the same level.

Let $v$ be a vertex of $T$. The stabilizer of $v$ is the group $\operatorname{Stab}(v)$ of elements of Aut $T$ which fix $v$. The $n$-th level stabilizer, written $\operatorname{Stab}(n)$, is the subgroup of Aut $T$ consisting of the elements that fix all the vertices of the $n$-th level. It is a normal subgroup of Aut $T$, isomorphic to $\prod_{v} \operatorname{Rist}(v)$ where $v$ ranges over the vertices of level $n$. The group Aut $T$ is the projective limit of the projective system $\operatorname{Aut} T / \operatorname{Stab}(n)$. If $T$ is locally finite then the groups $\operatorname{Aut} T / \operatorname{Stab}(n)$ are finite and Aut $T$ is profinite.

If $T \simeq X^{*}$ is a regular rooted tree, we shall often use the notation Aut $X^{*}$ for the automorphism group of $X^{*}$, and Aut $X^{n}$ for the finite quotient Aut $X^{*} / \operatorname{Stab}(n)$. 


\section{C.1. Introduction}

\section{The Automorphism Group of a Rooted Tree}

Let $T_{0}$ be a spherically homogeneous rooted tree with signature $\left(d_{i}\right)$. Let $T_{n}$ be a rooted tree isomorphic to a subtree of $T_{0}$ rooted at a vertex of level $n$ ( $T_{n}$ is independent of the choice of the vertex because $T_{0}$ is spherically homogeneous). There is a natural isomorphism Aut $T_{0} \simeq \operatorname{Aut} T_{1}$ S $\operatorname{Sym}\left(d_{0}\right)=\left(\operatorname{Aut} T_{1}\right)^{d_{0}} \rtimes \operatorname{Sym}\left(d_{0}\right)$, and more generally

$$
\begin{aligned}
\operatorname{Aut} T_{0} & \stackrel{\sim}{\longrightarrow} \operatorname{Aut} T_{n} \imath W_{n}, \\
g & \mapsto\left((g @ w)_{w \in L_{n}}, \sigma\right),
\end{aligned}
$$

where $W_{n}=\left(\cdots\left(\operatorname{Sym}\left(d_{n-1}\right) \prec \operatorname{Sym}\left(d_{n-2}\right)\right) \cdots\right) \prec \operatorname{Sym}\left(d_{0}\right)$ is a permutation group acting on $D_{n}=\prod_{i=0}^{n-1} d_{i}$ points, and $L_{n}$ is the set of vertices of level $n$. Note that the above defines $g @ w$ for any vertex $w$ of the tree as the image of $g$ under the composition Aut $T_{0} \rightarrow$ Aut $T_{n} \prec W_{n} \rightarrow\left(\text { Aut } T_{n}\right)^{D_{n}} \rightarrow$ Aut $T_{n}$, where the first and the last arrows are group homomorphisms, whereas the middle one is just a set map. We define $w * g=(1, \ldots, 1, g, 1, \ldots, 1)$ where $g$ appears in position $w$.

If $X^{*}$ is a $d$-ary rooted tree, then Aut $X^{*}$ is isomorphic to the iterated wreath product $\lim ^{n} \imath^{n} \operatorname{Sym}(d)$. In this case one can view $g \mapsto g @ w$ and $g \mapsto w * g$ as maps from Aut $X^{*}$ into itself. In this situation, a subgroup $G \leq \operatorname{Aut} X^{*}$ is called self-similar if $G @ w$ is contained in $G$ for all $w \in X^{*}$.

\section{Sylvan Representations}

Let $G$ be a group. A sylvan representation of $G$ is a group homomorphism $\rho: G \rightarrow$ Aut $T$ where $T$ is a locally finite rooted tree. The representation $\rho$ is faithful if $\rho$ is injective. The representation $\rho$ is level-transitive if the action of $\rho(G)$ is transitive on each level of $T$. Clearly $T$ must be spherically homogeneous for this condition to be satisfied.

Let $\rho$ be a faithful representation of $G$, and identify $G$ with its image in Aut $T$. Define $\operatorname{Rist}_{G}(v)=\operatorname{Rist}(v) \cap G$ and $G_{\# v}=\operatorname{Rist}_{G}(v) @ v$. Let $\operatorname{Rist}_{G}(n)$ be the group generated by $\operatorname{Rist}_{G}(v)$ for all $v$ of level $n$. The group $\operatorname{Rist}_{G}(n)$ is a normal subgroup of $G$. The representation $\rho$ is weakly branch if $\operatorname{Rist}_{G}(v)$ is non-trivial for all $v \in T$. The representation $\rho$ is branch if $\operatorname{Rist}_{G}(n)$ has finite index in $G$ for all $n \geq 0$. A group $G$ is (weakly) branch if there exists a faithful (weakly) branch representation of $G$. The representation $\rho$ is weakly regular branch if there is a subgroup $K \neq 1$ of $G$ such that $v * K \leq K$ for all $v \in X^{*}$. The representation $\rho$ is regular branch if moreover $\prod_{v \in X^{n}} v * K$ has finite index in $G$ for all $n \geq 0$. A group $G$ is (weakly) regular branch if there exists a faithful (weakly) regular branch representation of $G$. In this situation the subgroup $K$ shall be called a branching subgroup of $G$, and we shall denote by $X^{n} * K$ the product of all $v * K$ with $v \in X^{n}$. Note that, contrary to previous literature, we do not require level-transitivity as part of the definition.

Lemma C.1.2. Let $G$ be a (weakly) regular branch group. Then $G$ is (weakly) branch. 
Proof. Identify $G$ with its image in Aut $X^{*}$. Now $\operatorname{Rist}_{G}(v)$ obviously contains $v * K$, and thus a weakly regular branch group is regular branch. Further, $\operatorname{Rist}_{G}(n)$ contains $X^{n} * K$, which has finite index in $G$ if $G$ is regular branch.

Lemma C.1.3. Let $G$ be a regular branch group with faithful sylvan representation $\rho$. Then there is a unique maximal subgroup

$$
G_{\#}=\bigcap_{v \in X^{*}} G_{\# v}
$$

of $G$ that is a branching subgroup with respect to $\rho$.

Proof. Once again, identify $G$ with its image under $\rho$. Let $K$ be a branching subgroup. Then $v * K \leq K \leq G$ for all $v \in X^{*}$, so $v * K \leq \operatorname{Rist}_{G}(v)=v * G_{\# v}$. It follows that $K \leq G_{\# v}$ for all $v \in X^{*}$, so $K \leq G_{\#}$ and $G_{\#}$ is maximal.

Conversely, take $g \in G_{\#}$ and $v \in X^{*}$; then $g \in G_{\# u v}$ for all $u \in X^{*}$, so $v * g \in v * G_{\# u v} \leq G_{\# u}$, so $v * g \in G_{\#}$, and $G_{\#}$ is a branching subgroup.

A group $G \leq$ Aut $X^{*}$ is recurrent if $\left(\operatorname{Stab}_{G}(v)\right) @ v$ equals $G$ for all $v \in X^{*}$.

Lemma C.1.4. Let $G$ be a recurrent, regular branch group. Then $G_{\#} \unlhd G$.

Proof. We have $\operatorname{Rist}_{G}(v) \unlhd \operatorname{Stab}_{G}(v)$, so $G_{\# v} \unlhd \operatorname{Stab}_{G}(v) @ v=G$ for all $v \in$ $X^{*}$.

The following technical lemma has several important consequences.

Lemma C.1.5. Let $G \leq$ Aut $X^{*}$ be a self-similar, level-transitive, regular branch group. Then there is an integer $n$ so that $G_{\# u v}=G_{\# v}$ for all $u, v \in X^{*}$ with $|v| \geq n$.

Proof. Since $G$ is self-similar, we have $G_{\#} \leq G_{\# u v} \leq G_{\# v}$ for all $u, v \in X^{*}$. Since $G$ is level-transitive, $\left[G_{\# v}: G_{\#}\right]$ depends only on $|v|$; call this index $a_{m}$ if $|v|=m$. Then the $a_{m}$ form a non-increasing sequence of non-negative integers, so it must stabilize starting from some integer $n$.

Lemma C.1.6. For $v \in X^{*}$, the following properties are equivalent:

(i) $G_{\# v} \leq G_{\# u v}$ for all $u \in X^{*}$;

(ii) $u * \operatorname{Rist}_{G}(v) \leq \operatorname{Rist}_{G}(u v)$ for all $u \in X^{*}$;

(iii) $\operatorname{Rist}_{G}(v) \leq G_{\#}$.

Proof. (i) $\Rightarrow$ (iii) because $\operatorname{Rist}_{G}(v)=v * G_{\# v} \leq v * G_{\# u v} \leq G_{\# u}$ for all $u \in X^{*}$.

(iii) $\Rightarrow\left(\right.$ ii) because $G_{\#} \leq G$ by definition, so $u * \operatorname{Rist}_{G}(v) \leq G_{\#} \leq G$.

$($ ii $) \Rightarrow(i)$ because $u v * G_{\# v}=u * \operatorname{Rist}_{G}(v) \leq \operatorname{Rist}_{G}(u v)=u v * G_{\# u v}$.

Corollary C.1.7. Let $G$ be a self-similar, level-transitive, regular branch group. Then there exists $n \geq 0$ such that $X^{m} * G_{\#} \geq \operatorname{Rist}_{G}(m+n)$ for all $m \geq 0$. 


\section{C.2. The Congruence Subgroup Problem}

Proof. Let $n$ be given by Lemma C.1.5. Then $\operatorname{Rist}_{G}(v) \leq G_{\#}$ for all $v \in X^{n}$. Since $G$ is self-similar, $\operatorname{Rist}_{G}(u v) \leq u * \operatorname{Rist}_{G}(v) \leq u * G_{\#}$ for all $u \in X^{*}$.

Corollary C.1.8. Suppose moreover that $G$ is recurrent. Then $X^{m} * G_{\#}=$ $\operatorname{Rist}_{G}(m)$ for all $m \geq n$.

Proof. We have $G_{\#}=\bigcap_{v \in X^{*}} G_{\# v}$; and $G_{\# v} \unlhd G$, and depends only on $|v|$; so the $G_{\# v}$ are all equal to $G_{\#}$ when $|v| \geq n$. Then $\operatorname{Rist}_{G}(w)=w * G_{\# w}=w * G_{\#}$ when $|w| \geq n$.

We also define $\operatorname{Stab}_{G}(n)=\operatorname{Stab}(n) \cap G$. These are normal subgroups of $G$ and they form a filtration of $G$ with trivial intersection. Since $G / \operatorname{Stab}_{G}(n)$ is finite for all $n$, the group $G$ is residually finite. Note that Rist $(n)=\operatorname{Stab}(n)$ for the full automorphism group of the rooted tree, but in general the inclusion $\operatorname{Rist}_{G}(n) \leq \operatorname{Stab}_{G}(n)$ is proper.

\section{C.2 The Congruence Subgroup Problem}

\section{C.2.1 General Approach}

Consider a branch representation of an abstract group $\Gamma$ on a rooted tree $T$. We consider three topologies on $\Gamma$. We define these topologies by specifying a basis of neighbourhoods of the identity as follows.

\begin{tabular}{c|c|c} 
basis of neighbourhoods of 1 & name & completion \\
\hline$\left\{\operatorname{Stab}_{\Gamma}(n) \mid n \in \mathbb{N}\right\}$ & congruence topology & $\bar{\Gamma}$ \\
$\left\{\operatorname{Rist}_{\Gamma}(n) \mid n \in \mathbb{N}\right\}$ & branch topology & $\widetilde{\Gamma}$ \\
$\left\{N \mid N \unlhd_{f} \Gamma\right\}$ & profinite topology & $\widehat{\Gamma}$
\end{tabular}

The notation $N \unlhd_{f} \Gamma$ means $N$ is a normal subgroup of finite index in $\Gamma$. Since $\operatorname{Stab}_{\Gamma}(n)$ contains $\operatorname{Rist}_{\Gamma}(n)$ which has finite index in $\Gamma$, we deduce that the congruence topology is coarser than the branch topology, which is coarser than the profinite topology. It might happen that some of these topologies coincide but in general they are all different. These differences are characterized by the kernels of the natural maps:

$$
\begin{aligned}
\text { Congruence kernel }: \operatorname{ker}(\widehat{\Gamma} \rightarrow \bar{\Gamma}) & =\bigcap_{n \geq 0} \widehat{\operatorname{Stab}_{\Gamma}(n)}, \\
\text { Branch kernel }: \operatorname{ker}(\widehat{\Gamma} \rightarrow \widetilde{\Gamma}) & =\bigcap_{n \geq 0} \widehat{\operatorname{Rist}_{\Gamma}(n)}, \\
\text { Rigid kernel }: \operatorname{ker}(\widetilde{\Gamma} \rightarrow \bar{\Gamma}) & =\bigcap_{n \geq 0} \widehat{\operatorname{Stab}_{\Gamma}(n)},
\end{aligned}
$$

where, for a subgroup $H$ of $\Gamma$, we denote by $\widehat{H}$ its closure in $\widehat{\Gamma}$, and similarly we denote by $\widetilde{H}$ the closure of $H$ in $\widetilde{\Gamma}$. Another way of expressing this is the 
following:

$$
\begin{aligned}
& \operatorname{ker}(\widehat{\Gamma} \rightarrow \bar{\Gamma})=\lim _{\left(\operatorname{Stab}_{\Gamma}(n) N\right) / N,} \\
& \operatorname{ker}(\widehat{\Gamma} \rightarrow \widetilde{\Gamma})=\lim _{\left(\operatorname{Rist}_{\Gamma}(n) N\right) / N,} \\
& \operatorname{ker}(\widetilde{\Gamma} \rightarrow \bar{\Gamma})=\lim _{\operatorname{Stab}_{\Gamma}(n) / \operatorname{Rist}_{\Gamma}(n),}
\end{aligned}
$$

where $n$ ranges over the non-negative integers and $N$ over the finite-index subgroups of $\Gamma$. Consider $M \leq N$ two finite-index normal subgroups of $\Gamma$ and two integers $m \geq n$. The connecting $\operatorname{map} \operatorname{Stab}_{\Gamma}(m) M / M \rightarrow \operatorname{Stab}_{\Gamma}(n) N / N$ is given by

$$
\operatorname{Stab}_{\Gamma}(m) M / M \rightarrow \operatorname{Stab}_{\Gamma}(n) N / M \rightarrow \operatorname{Stab}(n) N / N
$$

where the first map is the inclusion and the second is the quotient map. The other connecting maps in (C.2.1) are defined analogously. We are in a position to state

Problem C.2.1 (Congruence subgroup problem). Compute the congruence, branch and rigid kernels.

A preliminary question of great importance however is as follows: Consider a branch group $\Gamma$.

Question C.2.2. Do any of the kernels depend on the branch representation of $\Gamma ?$

We shall address in this paper both of the questions.

\section{C.2.2 Branch Kernel}

\section{General Properties}

We extracted the following lemma from the proof of [Gri00, Theorem 4].

Lemma C.2.3. Consider an element $g$ of a group $\Gamma \leq$ Aut $T$ and let $v \leq w \in T$ be vertices so that $v$ is fixed by $g$, while $(w)^{g} \neq w$. Then the normal closure of $g$ in $\Gamma$ contains $\operatorname{Rist}_{\Gamma}(w)^{\prime}$.

Corollary C.2.4. Let $\Gamma \leq$ Aut $T$ be a level-transitive branch group and let $N \neq$ $\{1\}$ be a normal subgroup of $\Gamma$. Then $N$ contains $\operatorname{Rist}_{\Gamma}(n)^{\prime}$ for some $n \geq 0$.

Proof. Choose $1 \neq g \in N$ and vertices $v$ and $w$ as in the lemma. Then $N$ contains $\operatorname{Rist}_{\Gamma}(w)^{\prime}$. Because $\Gamma$ is level-transitive, $N$ also contains $\operatorname{Rist}_{\Gamma}(n)^{\prime}$ where $n$ is the depth of $w$.

We can drop the level-transitivity assumption under certain circumstances. We first need some definitions and one more lemma. Consider a regular rooted tree $X^{*}$. We say an element $g \in \operatorname{Aut} X^{*}$ is finite-state if the set $\left\{g @ w \mid w \in X^{*}\right\}$ is finite. Observe that all the elements of a group generated by finite-state elements are again finite-state. 


\section{C.2. The Congruence Subgroup Problem}

Lemma C.2.5. Let $\Gamma \leq$ Aut $X^{*}$ be a weakly branch group generated by a finite number of finite-state elements. Then $\Gamma$ does not fix any ray $w \in X^{\omega}$.

Proof. Let $S$ be a finite set of finite-state elements which generate $\Gamma$. The set of fixed points of an automorphism $s \in S$ is a regular language in $X^{*}$. The set $F$ of common fixed points of the elements of $S$, being the intersection of a finite number of regular languages, is again a regular language.

Thus by the Pumping Lemma [HU79, Chapter 3], either $F$ is finite and we are done, or there is an integer $n$ such that any $w \in F$ of length at least $n$ can be decomposed as $w=u v z$ with $|u v| \leq n$ and $|v|>0$, so that $u v^{k} z$ belongs to $F$ for all $k \geq 0$. This implies that the ray $u v^{\omega}$ is fixed by all the elements of $S$. We show that this leads to a contradiction.

Indeed, suppose that the elements of $S$, and thus $\Gamma$ itself, fix the ray $w=u v^{\omega}$. Then there are positive integers $k$ and $n$ such that for each generator $s \in S$ we have $s @ u v^{m}=s @ u v^{m+k}$ for all $m \geq n$. This is true for all the generators, hence it is true for the whole of $\Gamma$ : for each element $g \in \Gamma$, we have $g @ u v^{m}=g @ u v^{m+k}$ for all $m \geq n$. Consider now a vertex $x=u v^{m} w$ which is not a prefix of $u v^{n}$ for any $n$. If there existed $g \in G$ with $g @ x \neq 1$ and $g @ y=1$ for all $y$ not below $x$, then in particular $g @ u v^{m} \neq 1$, so $g @ u v^{m+k} \neq 1$, a contradiction.

A subset $C$ of $X^{*}$ is a cut-set if for each $w \in X^{\omega}$ there is exactly one element in $C$ which is a prefix of $w$.

Proposition C.2.6. Let $\Gamma$ be a weakly branch group generated by a finite number of finite-state elements. Then any subgroup $N$ of finite index in $\Gamma$ contains $\operatorname{Rist}_{\Gamma}(n)^{\prime}$ for some $n$.

Proof. $N$ contains a normal subgroup of finite index, so we assume $N$ is normal in $\Gamma$. First, we claim that there is a cut-set $C \subset X^{*}$ such that no element of $C$ is fixed by $N$. Indeed, the non-existence of such a cut-set is equivalent to $N$ fixing a ray, but this is impossible by Lemma C.2.5 (note that the hypotheses of the lemma are inherited by finite-index subgroups).

Next, for each element $w$ of $C$, we can find $g \in N$ which moves $w$. Let $v$ be the longest prefix of $w$ which is fixed by $g$ and let $x$ be such that $v x$ is a prefix of $w$. Then applying Lemma C.2.3, we see that $N$ contains $\operatorname{Rist}_{\Gamma}(v x)^{\prime}$, which in turn contains $\operatorname{Rist}_{\Gamma}(w)^{\prime}$. Thus $N$ contains $\operatorname{Rist}_{\Gamma}(n)^{\prime}$ where $n$ is the length of the longest word in $C$.

Lemma C.2.7. Let $\Gamma \leq$ Aut $T$ be a branch group and let $N \neq\{1\}$ be a normal subgroup of finite index in $\Gamma$. Then $N$ contains $\operatorname{Rist}_{\Gamma}(n)^{e}$ for some $n, e \geq 0$.

Proof. The group $\Gamma / N$ is finite, hence it has finite exponent $e$. In other words $\Gamma^{e} \leq N$, and since $\operatorname{Rist}_{\Gamma}(n)$ is a subgroup of $\Gamma$ we have the inclusions $\operatorname{Rist}_{\Gamma}(n)^{e} \leq$ $\Gamma^{e} \leq N$ for all $n \geq 0$.

Theorem C.2.8. Let $\Gamma \leq$ Aut $T$ be a branch group; assume either that $G$ is level-transitive, or that $G$ is generated by finitely many finite-state elements. The branch kernel $\mathcal{K}=\operatorname{ker}(\widehat{\Gamma} \rightarrow \widetilde{\Gamma})$ is Abelian. Moreover, if there is a positive integer 
e such that $\operatorname{Rist}_{\Gamma}(n) / \operatorname{Rist}_{\Gamma}(n)^{\prime}$ is of exponent dividing e for all $n \geq 0$, then $\mathcal{K}$ is of exponent dividing $e$.

Proof. Either Corollary C.2.4 or Proposition C.2.6, together with Lemma C.2.7, prove that the filters $\left\{N \mid N \unlhd_{f} \Gamma\right\}$ and $\left\{\operatorname{Rist}_{\Gamma}(n)^{\prime} \operatorname{Rist}_{\Gamma}(n)^{e} \mid n, e \in \mathbb{N}\right\}$ are cofinal. Hence $\mathcal{K}$ is the inverse limit of the system

$$
\operatorname{Rist}_{\Gamma}(n) /\left(\operatorname{Rist}_{\Gamma}(n)^{\prime} \operatorname{Rist}_{\Gamma}(n)^{e}\right) .
$$

These groups are Abelian, hence the limit is Abelian. If there is $e \geq 0$ such that $\operatorname{Rist}_{\Gamma}(n) / \operatorname{Rist}_{\Gamma}(n)^{\prime}$ is of exponent dividing $e$ for all $n \geq 0$ then $\mathcal{K}$ is the inverse limit of the system $\operatorname{Rist}_{\Gamma}(n) / \operatorname{Rist}_{\Gamma}(n)^{\prime}$, hence $\mathcal{K}$ is of exponent dividing $e$.

Recall that if $\widetilde{G}=\lim _{\longleftarrow} G / N$ is a profinite group and $A$ is a ring, then $A[[\widetilde{G}]]=$ $\lim A[G / N]$ is a profinite ring, called the complete group algebra of $\widetilde{G}$. See Sections $\overleftarrow{5.2}, 5.3$ and 5.5 of [RZ00] for more about free profinite modules, complete group algebras and complete tensor products.

Theorem $\mathbf{C}$ - E. Let $\Gamma \leq$ Aut $T$ be a level-transitive branch group. There is an Abelian group $A$ such that the branch kernel $\mathcal{K}=\operatorname{ker}(\widehat{\Gamma} \rightarrow \widetilde{\Gamma})$ is isomorphic to $\widehat{\mathbb{Z}}[[\widetilde{\Gamma}]] \widehat{\bigotimes}_{\widehat{\mathbb{Z}}\left[\left[\widetilde{\Gamma}_{w}\right]\right]}$ A qua profinite $A[[\widetilde{\Gamma}]]$-module. Here $\widetilde{\Gamma}_{w}$ is the stabilizer in $\widetilde{\Gamma}$ of a point $w \in \partial T$ of the boundary of the tree, where the action of $\widetilde{\Gamma}$ on $T$ is given by the natural map $\widetilde{\Gamma} \rightarrow \bar{\Gamma} \leq$ Aut $T$.

Moreover, $A$ can be explicitly computed as

$$
\lim _{\substack{v<w \\ k \geq 0}} \operatorname{Rist}_{\Gamma}(v) /\left(\operatorname{Rist}_{\Gamma}(v)^{\prime} \operatorname{Rist}_{\Gamma}(v)^{k}\right)
$$

Proof. The kernel $\mathcal{K}$ is the intersection $\bigcap_{n \geq 0} \widehat{\operatorname{Rist}_{\Gamma}(n)}$ by definition. Since $\Gamma$ is level-transitive, $\operatorname{Rist}_{\Gamma}(n)$ is the Cartesian product of $\operatorname{Rist}_{\Gamma}(v)$ for all $v$ of level n. Define $A_{w}=\bigcap_{v<w} \widehat{\operatorname{Rist}_{\Gamma}(v)}$, so that $\mathcal{K}$ embeds in the Cartesian product $\prod_{w \in \partial T} A_{w}$. By Proposition C.2.6 and Lemma C.2.7, we have

$$
A_{w}=\lim _{v<w, k \geq 0} \operatorname{Rist}_{\Gamma}(v) /\left(\operatorname{Rist}_{\Gamma}(v)^{\prime} \operatorname{Rist}_{\Gamma}(v)^{k}\right)
$$

In particular, the group $A_{w}$ is Abelian and it can be considered as a profinite ring.

Put $A_{v}:=\operatorname{Rist}_{\Gamma}(v) / \operatorname{Rist}_{\Gamma}(v)^{\prime}$ and consider it as a ring. Let $\Gamma_{v}$ be the stabilizer of the vertex $v$ in $\Gamma$. Then $\operatorname{Rist}_{\Gamma}(n) /\left(\operatorname{Rist}_{\Gamma}(n)\right)^{\prime}$ can be considered as a cyclic $A_{v} \Gamma$-module $\mathbb{Z}[\Gamma] \otimes_{\mathbb{Z}\left[\Gamma_{v}\right]} A_{v}$ with $v$ of length $n$. Therefore one has $\mathcal{K}=\bigcap_{n \geq 0} \widehat{\operatorname{Rist}_{\Gamma}(n)}=\underset{\longleftarrow}{\lim _{\operatorname{Rist}}(n)}=\varliminf_{\longleftarrow} \operatorname{Rist}_{\Gamma}(n) /\left(\operatorname{Rist}_{\Gamma}(n)^{\prime} \operatorname{Rist}_{\Gamma}(n)^{k}\right)$, and so $\mathcal{K}=\lim _{v<w, k \geq 0} \mathbb{Z}[\Gamma] \otimes_{\mathbb{Z}\left[\Gamma_{v}\right]}\left(A_{v} / k A_{v}\right)$. $\overleftarrow{U}_{\text {sing }}$ complete tensor products, one can rewrite this as $\widehat{\mathbb{Z}}[[\widehat{\Gamma}]] \widehat{\otimes}_{\widehat{\mathbb{Z}}\left[\left[\widehat{\Gamma}_{w}\right]\right]} A_{w}$, where $\widehat{\Gamma}_{w}$ is the inverse limit $\bigcap_{v<w} \widehat{\Gamma_{v}}$. Letting $\widehat{\Gamma}$ act on $T$ via the natural map $\widehat{\Gamma} \rightarrow \bar{\Gamma} \leq$ Aut $T$, the group $\widehat{\Gamma}_{w}$ is the stabilizer of $w$ in $\widehat{\Gamma}$.

Finally, by definition of $A_{w}$, the branch kernel acts trivially on it. Therefore one can simplify $\widehat{\mathbb{Z}}[[\widehat{\Gamma}]] \widehat{\otimes}_{\widehat{\mathbb{Z}}\left[\left[\widehat{\Gamma}_{w}\right]\right]} A_{w}=\widehat{\mathbb{Z}}[[\widetilde{\Gamma}]] \widehat{\otimes}_{\widehat{\mathbb{Z}}\left[\left[\widetilde{\Gamma}_{w}\right]\right]} A_{w}$. 


\section{C.2. The Congruence Subgroup Problem}

Remark C.2.9. As an $A$-module, $\widehat{\mathbb{Z}}[[\widetilde{\Gamma}]] \widehat{\otimes}_{\widehat{\mathbb{Z}}\left[\left[\widetilde{\Gamma}_{w}\right]\right]} A$ is isomorphic to $A\left[\left[\widetilde{\Gamma} / \widetilde{\Gamma}_{w}\right]\right] \simeq$ $A[[\partial T]]$. However, in general, there might be an action of $\widetilde{\Gamma}_{w}$ on $A$, as happens for example in Theorem C.3.29.

Corollary C.2.10. Let $\Gamma$ be a level-transitive, self-similar, regular branch group and let $K$ be its maximal branching subgroup. The branch kernel $\mathcal{K}=\operatorname{ker}(\widehat{\Gamma} \rightarrow \widetilde{\Gamma})$ is isomorphic to $\widehat{\mathbb{Z}}[[\widetilde{\Gamma}]] \widehat{\otimes}_{\widehat{\mathbb{Z}}\left[\left[\widetilde{\Gamma}_{w}\right]\right]} A$ where $A$ is the inverse limit of $\left\{K /\left(K^{\prime} K^{e}\right)\right\}$ with the maps induced by the composition

$$
K / K^{\prime} \simeq\left(K / K^{\prime}\right) \times\{1\} \times \cdots \times\{1\} \rightarrow\left(K / K^{\prime}\right)^{d} \rightarrow K /\left(K^{\prime}\right)^{d} \rightarrow K / K^{\prime} .
$$

In particular, if $K / K^{\prime}$ has finite exponent dividing e, then the branch kernel has exponent dividing $e$.

Proof. Corollary C.1.7 implies that the collections $\left\{X^{n} * K: n \geq 0\right\}$ and $\left\{\operatorname{Rist}_{\Gamma}(n)\right.$ : $n \geq 0\}$ are cofinal. Therefore one can write the branch kernel as $\lim \left(X^{n} *\right.$ $K) /\left(X^{n} *\left(K^{\prime} K^{e}\right)\right)$. Rewriting the proof above starting from this point yields the corollary.

Remark C.2.11. There is always an endomorphism $\sigma$ of $K$ defined by $g \mapsto$ $(g, 1, \ldots, 1)$. In the most favourable cases, $\sigma$ even extends to an endomorphism of the whole group $\Gamma$. The map $\sigma$ induces an endomorphism of $K / K^{\prime}$, and the connecting map above is just the inclusion $\sigma\left(K / K^{\prime}\right) \rightarrow K / K^{\prime}$. Thus one can rewrite the definition of $A$ as $A=\lim _{e \geq 0, n \geq 0} \sigma^{n}\left(K /\left(K^{\prime} K^{e}\right)\right)$. If $K / K^{\prime}$ is finite, the previous expression simplifies to $A=\bigcap_{n \geq 0} \sigma^{n}\left(K / K^{\prime}\right)$.

Corollary $\mathbf{C}$ - F. Let $\Gamma$ be a just-infinite, level-transitive, self-similar, regular branch group. The branch kernel $\mathcal{K}=\operatorname{ker}(\widehat{\Gamma} \rightarrow \widetilde{\Gamma})$ has finite exponent.

Proof. Indeed $K^{\prime}$ is normal in $\Gamma$, and hence has finite index in $\Gamma$, and a fortiori in $K$. The claim follows from Corollary C.2.10.

\section{Independence of the Representation}

We use a result of Abért [Abé05]. Let $G$ be a group acting on a set $X$. We say that $G$ separates $X$ if for any finite subset $Y \subseteq X$ the pointwise stabilizer $G_{Y}$ does not stabilize any point outside $Y$. Recall that a group $G$ satisfies a group law if there is a non-trivial element $w$ in a free group $F$ which maps to the identity under any homomorphism $F \rightarrow G$.

Theorem C.2.12 ([Abé05, Theorem 1]). If a group $G$ separates $X$ then $G$ does not satisfy any group law.

Corollary C.2.13. Let $\Gamma \leq$ Aut $T$ be a level-transitive branch group and let $N$ be a non-trivial normal subgroup of $\Gamma$. Then $N$ cannot satisfy any group law.

Proof. If a non-trivial normal subgroup $N$ of $\Gamma$ satisfies a law, then $\Gamma$ satisfies a law. Indeed by Corollary C.2.4 the group $N$ contains the commutator subgroup of the rigid stabilizer of some level, so this rigid stabilizer must satisfy a law. 
However, it is of finite index in $\Gamma$, so $\Gamma$ satisfies a law. This shows that in our case it is sufficient to prove that $\Gamma$ satisfies no law.

$\Gamma$ acts on the boundary $\partial T$ of $T$ and by Theorem C.2.12 it is enough to show that $\Gamma$ separates $\partial T$. Consider a finite subset $Y \subset \partial T$ and a point $w \in \partial T \backslash Y$. Choose a prefix $v$ of $w$ such that no descendant of $v$ is in $Y$. Then $\operatorname{Rist}_{\Gamma}(v)$ is nontrivial because $\Gamma$ is branch and level-transitive, thus we can choose a non-trivial element $g \in \operatorname{Rist}_{\Gamma}(v)$. The automorphism $g$ moves a vertex $u \geq v$. Conjugating $g$ by an element $h \in \Gamma$ which moves $u$ to a prefix of $w$ gives an element $g^{\prime}$ in $\operatorname{Rist}_{\Gamma}(v)$ which moves $w$, as needed.

We are now able to prove Theorem $\mathrm{C}-\mathrm{D}$, which shows that for a leveltransitive, branch representation, the branch kernel is contained in the congruence kernel of any other level-transitive, branch representation.

We do not know any example of a group having representations with different rigid kernels.

Theorem $\mathbf{C}-\mathbf{D}$. Let $\Gamma$ be a level-transitive branch group and consider two leveltransitive branch representations $\rho_{i}: \Gamma \rightarrow$ Aut $T_{i}$ of $\Gamma$ with $i=1,2$. Write $\widetilde{\Gamma}_{i}$ and $\bar{\Gamma}_{i}$ for the corresponding branch and congruence completion and denote the branch kernels by $\mathcal{K}_{i}=\operatorname{ker}\left(\widehat{\Gamma} \rightarrow \widetilde{\Gamma}_{i}\right)$.

Then $\mathcal{K}_{i}$ is in the congruence kernel $\operatorname{ker}\left(\widehat{\Gamma} \rightarrow \bar{\Gamma}_{j}\right)$ for all $i, j \in\{1,2\}$.

Proof. $\mathcal{K}_{i}$ is a normal subgroup of $\widehat{\Gamma}$ and it is Abelian by Theorem C.2.8. The image $\overline{\mathcal{K}}_{1}^{(2)}$ of $\mathcal{K}_{1}$ in $\bar{\Gamma}_{2}$ is a normal subgroup and it is Abelian too. Because of Corollary C.2.13 the group $\overline{\mathcal{K}}_{1}^{(2)}$ is trivial because $\bar{\Gamma}_{2}$ is branch. Therefore $\mathcal{K}_{1}$ lies in the kernel of the map $\widehat{\Gamma} \rightarrow \bar{\Gamma}_{2}$. The argument is clearly symmetric, and so $\mathcal{K}_{2}$ is in the kernel of the map $\widehat{\Gamma} \rightarrow \bar{\Gamma}_{1}$.

\section{C.2.3 Rigid Kernel}

Here we only consider the case of self-similar, regular branch groups.

\section{General Properties}

Theorem C.2.14. Let $\Gamma$ be a level-transitive, self-similar, regular branch group. Then the kernel of $\widetilde{\Gamma} \rightarrow \bar{\Gamma}$ has finite exponent.

Proof. Let $K$ be the maximal branching subgroup of $\Gamma$. By Corollary C.1.7 the collections $\left\{X^{n} * K: n \geq 0\right\}$ and $\left\{\operatorname{Rist}_{\Gamma}(n): n \geq 0\right\}$ are cofinal. Therefore the rigid kernel can be computed as $\lim _{-} \operatorname{Stab}_{\Gamma}(n) / \operatorname{Core}_{\Gamma}\left(X^{n} * K\right)$, where for a subgroup $H \leq \Gamma$ we define $\operatorname{Core}_{\Gamma}(H) \overleftarrow{=} \bigcap_{g \in \Gamma} H^{g}$.

Note that $\operatorname{Core}_{\Gamma}\left(X^{n} * K\right)$ contains $X^{n} * \operatorname{Core}_{\Gamma}(K)$ because the latter is contained in $X^{n} * K$ and normal in $\Gamma$. The image of $\operatorname{Stab}_{\Gamma}(n)$ through the wreath decomposition $\Gamma \rightarrow \Gamma^{X^{n}} \rtimes\left(\Gamma / \operatorname{Stab}_{\Gamma}(n)\right)$ is contained in $\Gamma^{X^{n}}$. Therefore $\operatorname{Stab}_{\Gamma}(n) / \operatorname{Core}_{\Gamma}\left(X^{n} * K\right)$ is a subquotient of $\left(\Gamma / \operatorname{Core}_{\Gamma}(K)\right)^{X^{n}}$ for all $n \geq 0$. This proves that the rigid kernel of $\Gamma$ has exponent bounded by $e$ where $e$ is the exponent of $\Gamma / \operatorname{Core}_{\Gamma}(K)$, which is finite by Lemma C.1.2 and Corollary C.1.7. 


\section{C.3. Examples}

\section{Independence of the Representation}

Theorem $\mathbf{C}-\mathbf{A}$. Let $\Gamma$ be a group and consider two level-transitive, self-similar, regular branch representations $\Gamma \rightarrow$ Aut $T_{i}$ with corresponding closures $\bar{\Gamma}_{i}$. The congruence kernel $\mathcal{K}_{1}=\operatorname{ker}\left(\widehat{\Gamma} \rightarrow \bar{\Gamma}_{1}\right)$ is equal to the congruence kernel $\mathcal{K}_{2}=$ $\operatorname{ker}\left(\widehat{\Gamma} \rightarrow \bar{\Gamma}_{2}\right)$.

Proof. Combining Theorems C.2.8 and C.2.14 we see that $\mathcal{K}_{1}$ and $\mathcal{K}_{2}$ are both extensions of an Abelian group by a group of finite exponent. Thus $\mathcal{K}_{1}$ and $\mathcal{K}_{2}$ satisfy a group law (big powers commute). Corollary C.2.13 shows that the image of $\mathcal{K}_{1}$ in $\bar{\Gamma}_{2}$ is trivial, thus $\mathcal{K}_{1} \leq \mathcal{K}_{2}$ and vice versa.

\section{C.3 Examples}

We describe in Sections C.3.1 and C.3.2 some groups with trivial rigid kernel, but with non-trivial branch kernel. In contrast, we describe in Section C.3.3 the Hanoi tower group, which has both branch and rigid kernel non-trivial.

\section{C.3.1 Pervova's Examples}

Pervova [Per07] has constructed the first examples of groups acting on rooted trees which fail to have the congruence subgroup property, i.e. the kernel of $\widehat{\Gamma} \rightarrow \bar{\Gamma}$ is non-trivial. Her examples act on a $p$-ary rooted tree where $p$ is an odd prime. We detail the congruence kernels only for $p=3$ for simplicity but the other examples are similar.

Define the rooted tree automorphisms $a, b, c$ via the recursions

$$
a=\sigma_{123}, \quad b=\left(a, a^{-1}, b\right), \quad c=\left(c, a, a^{-1}\right) .
$$

We let $\Gamma$ be the group generated by $a, b, c$. Considering furthermore the element $d=(d, d, d) \sigma_{123}$, we see that $\langle a, c\rangle=\langle a, b\rangle^{d}$ is the infinite torsion group considered by Gupta and Sidki [GS83b]. In fact, the same proof shows that $\Gamma$ is a torsion group. Clearly the group $\Gamma$ is self-similar, level-transitive and recurrent.

Lemma C.3.1. $\Gamma$ is regular branch, with branching subgroup $\Gamma^{\prime}$.

Proof. It suffices to check the computations

$$
\begin{aligned}
& 1 *[a, b]=\left[b^{-a} b^{-a^{2}}, b^{-1} b^{-a}\right], \\
& 1 *[a, c]=\left[c^{-1} c^{-a}, c^{-a^{2}} c^{-1}\right], \\
& 1 *[b, c]=\left[b^{a}, c\right] .
\end{aligned}
$$

We have [Per07, Lemma 1.4]:

$$
\Gamma / \Gamma^{\prime} \simeq C_{3} \times C_{3} \times C_{3},
$$

but on the other hand it is easy to see that $\bar{\Gamma} / \bar{\Gamma}^{\prime} \simeq C_{3} \times C_{3}$; indeed $c^{-1} b=$ $[a, b](1 *[a, b])\left(1^{2} *[a, b]\right) \cdots$ belongs to $\bar{\Gamma}^{\prime}$. This proves that $\Gamma^{\prime}$ is not a congruence subgroup. 
Proposition C.3.2 ([Per07, Proposition 1.3]). We have the inclusion $\Gamma^{\prime} \leq \Gamma_{\# v}$ for all vertices $v$. $b^{-1} c$.

From this we show slightly more. Let $K$ be the group generated by $\Gamma^{\prime}$ and

Proposition C.3.3. We have the inclusion $K \leq \Gamma_{\# v}$ for all vertices $v$.

Proof. It is enough to show that $K$ contains $1 *\left(b^{-1} c\right)$. A direct computation shows $[a, b] b^{-1} c=b^{-a} c=1 *\left(b^{-1} c\right) \in K$.

Proposition C.3.4. The groups $\widetilde{\Gamma}$ and $\bar{\Gamma}$ coincide.

Proof. We show that $K$ contains $\operatorname{Stab}_{\Gamma}(2)$. This proves that the topologies induced by $\left\{\operatorname{Stab}_{\Gamma}(n) \mid n \in \mathbb{N}\right\}$ and $\left\{\operatorname{Rist}_{\Gamma}(n) \mid n \in \mathbb{N}\right\}$ are the same.

Clearly $K$ has index 9 in $\Gamma$, and a direct computation shows that $\pi_{2}(\Gamma) / \pi_{2}(K)$ is a group of order 9 , where $\pi_{2}$ is the canonical map $\Gamma \rightarrow \Gamma / \operatorname{Stab}_{\Gamma}(2)$. Thus $K$ contains $\operatorname{Stab}_{\Gamma}(2)$.

Theorem C.3.5 ([Per07, Theorem 3.2]). Any finite index subgroup $N$ of $\Gamma$ contains $X^{n} * \Gamma^{\prime}$ for some $n$.

In [Per07, Theorem 3.4], the congruence kernel of $\Gamma$ is described as an inverse limit of elementary Abelian p-groups. Using our Corollary C.2.10, the structure of the kernel follows from an easy computation, and we get the $\bar{\Gamma}$-module action almost for free.

Corollary C.3.6. The branch and congruence kernels of $\Gamma$ are $(\mathbb{Z} / 3 \mathbb{Z})\left[\left[X^{\omega}\right]\right]$.

Proof. Both kernels are equal by Proposition C.3.4, so we concentrate on the former. We use Corollary C.2.10 to deduce that the congruence kernel is $\widehat{\mathbb{Z}}[[\widetilde{\Gamma}]] \widehat{\otimes}_{\widehat{\mathbb{Z}}\left[\left[\widetilde{\Gamma}_{w}\right]\right]} A$ where $A$ is the inverse limit of $K / \Gamma^{\prime}$ with the connecting map

$$
K / \Gamma^{\prime} \rightarrow K / \Gamma^{\prime} \times\{1\} \times\{1\} \rightarrow K /\left(\Gamma^{\prime}\right)^{3} \rightarrow K / \Gamma^{\prime} .
$$

$K / \Gamma^{\prime}$ is a cyclic group of order 3 generated by $b^{-1} c$, and a straightforward computation shows $1 *\left(b^{-1} c\right)=[a, b] b^{-1} c \equiv b^{-1} c\left(\bmod \Gamma^{\prime}\right)$. Therefore $A$ is the inverse limit of $\mathbb{Z} / 3 \mathbb{Z}$ with the connecting map being the identity, whence $A \simeq \mathbb{Z} / 3 \mathbb{Z}$. Moreover the action of $\operatorname{Stab}_{\Gamma}(v)$ on $v *\left(K / \Gamma^{\prime}\right)$ is trivial. Therefore $\widehat{\mathbb{Z}}[[\widetilde{\Gamma}]] \widehat{\otimes}_{\widehat{\mathbb{Z}}\left[\left[\widetilde{\Gamma}_{w}\right]\right]} A=(\mathbb{Z} / 3 \mathbb{Z})\left[\left[\widetilde{\Gamma} / \widetilde{\Gamma}_{w}\right]\right]=(\mathbb{Z} / 3 \mathbb{Z})\left[\left[\bar{\Gamma} / \bar{\Gamma}_{w}\right]\right]=(\mathbb{Z} / 3 \mathbb{Z})\left[\left[X^{\omega}\right]\right]$.

\section{C.3.2 The Twisted Twin of Grigorchuk's Group}

We sketch here the computation of the congruence kernel for a new example of group, which is a twisted relative of Grigorchuk's first group [Gri80]. More details will appear in [BS09].

We define the automorphisms of the binary tree

$$
\begin{array}{llrl}
a & =\sigma_{12}, & b & =(c, a) \\
c & =(a, d), & d & =(1, b) .
\end{array}
$$




\section{C.3. Examples}

We let $H$ be the group generated by $a, b, c, d$. The group $H$ is a just-infinite, torsion, level-transitive, self-similar, recurrent, regular branch group.

Define $K$ as the normal closure of $\{[a, b],[b, c],[c, d],[d, b], b c d\}$ in $H$. We have

Proposition C.3.7 ([BS09]). The group $K$ contains $K \times K$, and $K$ contains $\operatorname{Stab}_{H}(3)$.

Corollary C.3.8. The groups $\widetilde{H}$ and $\bar{H}$ coincide.

Proposition C.3.9 ([BS09]). Any finite-index normal subgroup $N$ of $H$ contains $X^{n} *[K, H]$ for some $n \geq 0$.

Let $\sigma$ be the endomorphism of $H$ induced by $a \mapsto c, b \mapsto d^{a}, c \mapsto b, d \mapsto c^{a}$.

Theorem C.3.10. The branch and congruence kernels of $H$ are $(\mathbb{Z} / 4 \mathbb{Z})\left[\left[X^{\omega}\right]\right]$.

Proof. Both kernels are equal by Corollary C.3.8, so we concentrate on the former. We shall compute the group $A$ from Corollary C.2.10 using an improvement of Remark C.2.11. Namely, we have an endomorphism $\sigma$ of $H$ inducing $g \mapsto(g, 1)$ on $K$. Moreover, by Proposition C.3.9, we only have to consider the group $K /[K, H]$. This group inherits the endomorphism $\sigma$, and thus we have $A=\bigcap_{n \geq 0} \sigma^{n}(K /[K, H])$. It is proved in [BS09] that the group $K /[K, H]$ is isomorphic to $C_{4} \times C_{2}$, the $C_{4}$ being generated by $b c d$ and the $C_{2}$ by $[a, b]$. Further computations show that $\sigma$ sends $b c d$ to $b c d[a, b]$ and $[a, b]$ to $(b c d)^{2} \bmod [K, H]$. Thus $\sigma(K /[K, H])$ is a cyclic group of order 4 , generated by $b c d[a, b]$, and it is invariant under $\sigma$ (it is not fixed pointwise, however).

Finally, the action by conjugation of $H$ on $K /[K, H]$ is obviously trivial.

\section{C.3.3 The Hanoi Tower Group}

This group was introduced by Sunic and Grigorchuk; see [GŠ06] or [OWR06, page 1477]. It models the "Towers of Hanoi" game in the sense that the graph of the action of $\Gamma$ on $X^{n}$ describes the space of moves of $n$ disks on a 3-peg game.

We define the following automorphisms of the 3 -ary rooted tree:

$$
a=(a, 1,1) \sigma_{23}, \quad b=(1, b, 1) \sigma_{13}, \quad c=(1,1, c) \sigma_{12} .
$$

We let $\Gamma$ be the group generated by $a, b, c$. Since the definition is symmetric in $a, b, c$, any permutation of the letters $a, b, c$ yields an automorphism of $\Gamma$.

The Abelianization $\Gamma / \Gamma^{\prime}$ of $\Gamma$ is isomorphic to $C_{2} \times C_{2} \times C_{2}$, but we have $\bar{\Gamma} / \bar{\Gamma}^{\prime} \simeq C_{2}$. Therefore $\Gamma^{\prime}$ is not a congruence subgroup. However, the situation is more subtle than in the previous examples.

Theorem C.3.11. - The kernel of $\widehat{\Gamma} \rightarrow \widetilde{\Gamma}$ is free profinite Abelian;

- The kernel of $\widetilde{\Gamma} \rightarrow \bar{\Gamma}$ is a Klein group of order 4;

- The kernel of $\widehat{\Gamma} \rightarrow \bar{\Gamma}$ is metabelian and torsion-free, but is not nilpotent. 


\section{A Presentation of $\Gamma$}

The main tool for this section is a recursive presentation of $\Gamma$.

We recall the general strategy in obtaining presentations by generators and relations for self-similar groups; for more details see [Bar03] or [Sid87].

We note that $\Gamma$ is contracting with nucleus $N=\{1, a, b, c\}$. By definition, this means that for any $g \in \Gamma$, there is an $n \geq 0$ so that $g @ w$ is in $N$ for all $w$ of length at least $n$. The only relations of length $\leq 3$ among elements of $N$ are $a^{2}=b^{2}=c^{2}=1$. We consider thus the group $F=\left\langle a, b, c \mid a^{2}, b^{2}, c^{2}\right\rangle$. The decomposition map $\Gamma \rightarrow \Gamma$ ? $\operatorname{Sym}(X)$ restricts to a map $N \rightarrow N^{X} \times \operatorname{Sym}(X)$, which induces a homomorphism $\psi: F \rightarrow F$ ? $\operatorname{Sym}(X)$. Set $K_{0}=1 \triangleleft F$ and $K_{n+1}=\psi^{-1}\left(K_{n}^{X}\right)$ for all $n \geq 0$.

Lemma C.3.12. $\Gamma=F / \bigcup_{n} K_{n}$.

Proof. By our choice of relations in $F$, the decomposition $\psi$ is contracting on $F$, with nucleus $\{1, a, b, c\}$. Given $w \in F$ : if $w$ belongs to $K_{n}$ for some $n$, then it is clear that $w$ is trivial in $\Gamma$. Conversely, if $w$ is trivial in $\Gamma$, there exists $n$ such that all $w$ 's level- $n$ states belong to $N$ and act trivially; so they are all 1 ; so $w$ belongs to $K_{n}$.

It is easy, using the Reidemeister-Schreier rewriting method, to construct a normal generating set for $K_{1}$. Indeed, $\psi$ induces an injective map $F / K_{1} \rightarrow$ $F$ ? $\operatorname{Sym}(X)$, whose image has index 32 . We introduce some notation:

$$
d=[a, b], \quad e=[b, c], \quad f=[c, a], \quad g=d^{c}, \quad h=e^{a}, \quad i=f^{b} .
$$

Then we obtain quite explicitly

$$
\begin{aligned}
K_{1}=\left\langle d^{-1} e f i^{-1} g e^{-1}, h e^{-1} d^{-1} f d i^{-1}, e^{-1} g^{-1} f^{-1} e g f,\right. \\
\\
\left.\quad e^{-1} d h e^{-2} d^{-1} h^{2}, h g d^{-2} f^{-1} g f e^{-1}\right\rangle^{F} .
\end{aligned}
$$

We also consider the homomorphism $\tau: F \rightarrow F$ defined by

$$
a \mapsto a, \quad b \mapsto b^{c}, \quad c \mapsto c^{b} .
$$

Lemma C.3.13. Let $\tau^{\prime}$ be any homomorphism $F \rightarrow D_{\infty}=\left\langle x, y \mid x^{2}, y^{2}\right\rangle$ that sends a to 1 and $b, c$ to conjugates of $x, y$ respectively. Then $\tau^{\prime}\left(K_{1}\right)=1$.

Proof. We have $\tau^{\prime}(d)=\tau^{\prime}(f)=\tau^{\prime}(g)=\tau^{\prime}(i)=1$, and $\tau^{\prime}(e)=\tau^{\prime}(h)$. It remains to check that each normal generator of $K_{1}$ contains (with sign) as many $e$ 's as $h$ 's.

It follows that, for all normal generators $r$ of $K_{1}$, we have $\psi(\tau(r))=(r, 1,1)$. Therefore, $K_{n}$ is normally generated by $\bigcup_{i<n} \tau^{i}\left(K_{1}\right)$ for all $n>0$. We conclude:

\section{Proposition C.3.14.}

$$
\left.\Gamma=\langle a, b, c| a^{2}, b^{2}, c^{2}, \tau^{n}\left(w_{1}\right), \tau^{n}\left(w_{2}\right), \tau^{n}\left(w_{3}\right), \tau^{n}\left(w_{4}\right), \tau^{n}\left(w_{5}\right) \text { for all } n \geq 0\right\rangle,
$$

where $w_{1}, \ldots, w_{5}$ are the five normal generators of $K_{1}$ above. 


\section{C.3. Examples}

Remark C.3.15. One can check that $w_{5}$ is a consequence of $w_{1}, w_{2}, w_{3}, w_{4}, \tau\left(w_{1}\right)$ in $F$, and thus one has the simpler presentation

$$
\left.\Gamma=\langle a, b, c| a^{2}, b^{2}, c^{2}, \tau^{n}\left(w_{1}\right), \tau^{n}\left(w_{2}\right), \tau^{n}\left(w_{3}\right), \tau^{n}\left(w_{4}\right) \text { for all } n \geq 0\right\rangle .
$$

We deduce from Proposition C.3.14 a short proof of a result by Sunic:

Proposition C.3.16 (Sunic). The map $a \mapsto 1, b \mapsto b, c \mapsto c$ extends to an endomorphism of $\Gamma$.

Proof. This map is an instance of a map $\tau^{\prime}$ in the lemma; all relators of $\Gamma$ are mapped to one under it.

\section{The Branch Kernel of $\Gamma$}

Proposition C.3.17. The group $\Gamma^{\prime} / \Gamma^{\prime \prime}$ is isomorphic to $\mathbb{Z}^{3} \times C_{3}$, where $\mathbb{Z}^{3}$ is generated by $[a, b],[b, c],[c, a]$ and $C_{3}$ is generated by $[[a, b], c]$.

Proof. $\Gamma^{\prime}$ is generated by $d, e, f, g, h, i$ in the notation introduced above. The exponent counts of these generators in the relations $r_{1}, \ldots, r_{5}$ are given by the matrix

$$
\left(\begin{array}{llllll}
-1 & & 1 & 1 & & -1 \\
& -1 & 1 & & 1 & -1 \\
& & & & & \\
& -3 & & & 3 & \\
-2 & -1 & & 2 & 1 &
\end{array}\right) \rightsquigarrow \cdots \rightsquigarrow\left(\begin{array}{llllll}
1 & & 2 & -1 & & -2 \\
& 1 & 2 & & -1 & -2 \\
& & 3 & & & -3
\end{array}\right) ;
$$

its Smith normal form has two ones and a three on its diagonal; the three generators of infinite rank of $\Gamma^{\prime} / \Gamma^{\prime \prime}$ may be chosen as $d, e, f$, while $e h^{-1}$ generates a $C_{3}$ therein, and equals $d g^{-1}$ and $f i^{-1}$.

Proposition C.3.18. The branch kernel of $\Gamma$ is isomorphic to $\widehat{\mathbb{Z}}^{3}\left[\left[X^{\omega}\right]\right]$.

Proof. We consider the group $\Gamma^{\prime} / \Gamma^{\prime \prime} \simeq \mathbb{Z}^{3} \times C_{3}$ with the endomorphism defined by the composition

$$
\Gamma^{\prime} / \Gamma^{\prime \prime} \stackrel{\sim}{\rightarrow} \Gamma^{\prime} / \Gamma^{\prime \prime} \times\{1\} \times\{1\} \rightarrow \Gamma^{\prime} /\left(\Gamma^{\prime \prime}\right)^{3} \rightarrow \Gamma^{\prime} / \Gamma^{\prime \prime},
$$

and we would like to compute the $\operatorname{limit} \underset{\lim }{\longleftarrow} \Gamma^{\prime} / \Gamma^{\prime \prime}$. Recall that $d g^{-1}=e h^{-1}=$ $f i^{-1} \bmod \Gamma^{\prime \prime}$, and note that in $\Gamma$, the relation $\left(d g^{-1}, 1,1\right)=h^{-1} d e^{3} g^{-1} f h^{-1} f^{-1} e^{-1}$ holds. This element is equal to $d g^{-1} e^{2} h^{-2}=1 \bmod \Gamma^{\prime \prime}$.

Next, we compute $(d, 1,1)=h^{-1} d e$, and similarly $(e, 1,1)=e^{-1} i f^{-1}$ and $(f, 1,1)=e f h^{-1}$ in $\Gamma$. These elements are equal to $d e h^{-1}, e^{-2} h$ and $f e h^{-1}$ respectively, $\bmod \Gamma^{\prime \prime}$. This shows that the endomorphism of $\Gamma^{\prime} / \Gamma^{\prime \prime}$ is given by left multiplication by the matrix

$$
\left(\begin{array}{cccc}
1 & 0 & 0 & 0 \\
0 & -1 & 0 & 0 \\
0 & 0 & 1 & 0 \\
1 & -1 & 1 & 0
\end{array}\right)
$$

with rows and columns indexed by $\left\{d, e, f, e h^{-1}\right\}$. Thus, using Corollary C.2.10, we have proved the proposition. 


\section{The Rigid Kernel of $\Gamma$}

Let $A$ be the group $\Gamma / \Gamma^{\prime} \simeq(\mathbb{Z} / 2 \mathbb{Z})^{3}$, generated by $\bar{a}, \bar{b}, \bar{c}$. Viewing $A$ as a ring (with component-wise multiplication), we denote by $A\left[\left[X^{\omega}\right]\right]$ the free profinite $A$-module on the profinite space $X^{\omega}=\lim _{\longleftarrow} X^{n}$. Then $A\left[\left[X^{\omega}\right]\right]$ is in particular an Abelian profinite group, and it is endowed with a natural action of Aut $X^{*}$. If $m=\sum_{w \in X^{\omega}} w * m_{w}$ is an element of $A\left[\left[X^{\omega}\right]\right]$ and $g$ is in Aut $X^{*}$, then the action of $g$ on $m$ is given by

$$
g \cdot m=\sum_{w \in X^{\omega}} w^{g^{-1}} * m_{w}
$$

Thus we can consider the extension $W=A\left[\left[X^{\omega}\right]\right] \rtimes$ Aut $X^{*}$ which is again a profinite group. It is straightforward to check that $W$ is isomorphic (as a profinite group) to the inverse limit of the groups $W_{n}=A\left[X^{n}\right] \rtimes$ Aut $X^{n}$ with the natural maps $W_{n+1} \rightarrow W_{n}$ given by

$$
\left(\sum_{w \in X^{n}, x \in X} w x * m_{w x}, g\right) \mapsto\left(\sum_{w \in X^{n}, x \in X} w * m_{w x}, g \mid X^{n}\right) .
$$

We define the following elements of $W$ :

$$
\alpha=\left(1^{\omega} * \bar{a}, a\right), \quad \beta=\left(2^{\omega} * \bar{b}, b\right), \quad \gamma=\left(3^{\omega} * \bar{c}, c\right),
$$

and we let $G$ be the subgroup of $W$ generated by $\alpha, \beta, \gamma$.

Proposition C.3.19. The natural epimorphism $W \rightarrow$ Aut $X^{*}$ restricts to an isomorphism $\pi: G \rightarrow \Gamma$.

Proof. The map $\pi$ is obviously an epimorphism. To complete the proof, we construct an inverse $\psi: \Gamma \rightarrow G$. To this end we set $\psi(g)=\left(\psi_{M}(g), g\right)$ and we still have to define $\psi_{M}(g)$. We first set

$$
\psi(a)=\alpha, \quad \psi(b)=\beta, \quad \psi(c)=\gamma,
$$

and $\psi_{M}(1)=0$. It is readily checked that the formula

$$
\psi_{M}(g)=\sum_{w \in X^{n}} w * \psi_{M}(g @ w)
$$

holds for all $n \geq 0$ and $g \in N$. By imposing it to hold for all $g$ in $\Gamma$, the formula defines $\psi_{M}(g)$ for all $g \in \Gamma$ because of the contracting property. Indeed for an element $g \in \Gamma$, we can choose $n$ big enough so that $g @ w$ is in $N$ for all $w \in X^{n}$. Then $\psi_{M}(g)=\sum_{w \in X^{n}} w * \psi_{M}(g @ w)$ is well-defined. We have thus defined $\psi(g)=\left(\psi_{M}(g), g\right)$ for all $g \in \Gamma$.

Obviously we have $\pi \circ \psi=\mathrm{id}_{\Gamma}$, and the composition $\psi \circ \pi$ is the identity when restricted to $\psi(N)$. Since $\psi(N)$ generates $G$, we will show by induction on the length of $g$ that the relation $\psi(\pi(g))=g$ holds for all $g \in G$ (we consider the length of $g$ in the word metric of $G$ with respect to the generating set $\psi(N)$ ). 


\section{C.3. Examples}

Consider $g=(m, \bar{g}) \in G$ and $h=(n, \bar{h}) \in \psi(N)$. Then we have $\pi(g h)=\bar{g} \bar{h}$ and we must show $\psi(\bar{g} \bar{h})=g h=(m+\bar{g} \cdot n, \bar{g} \bar{h})$. Thus all we need to prove is $\psi_{M}(\bar{g} \bar{h})=m+\bar{g} \cdot n$. Choose $k$ big enough so that $\bar{g} @ w$ and $(\bar{g} \bar{h}) @ w$ all belong to $N$ for all $w \in X^{k}$. Then by definition of $\psi$ we have

$$
\begin{gathered}
\psi_{M}(\bar{g} \bar{h})=\sum_{w \in X^{k}} w * \psi_{M}((\bar{g} \bar{h}) @ w)=\sum_{w \in X^{k}} w * \psi_{M}\left(\bar{g} @ w \bar{h} @ w^{\bar{g}}\right) \\
=\sum_{w \in X^{k}} w *\left(\psi_{M}(\bar{g} @ w)+\psi_{M}\left(\bar{h} @ w^{\bar{g}}\right)\right) \\
=\sum_{w \in X^{k}} w * \psi_{M}(\bar{g} @ w)+\sum_{w \in X^{k}} w^{\bar{g}^{-1}} * \psi_{M}(\bar{h} @ w)=m+\bar{g} \cdot n .
\end{gathered}
$$

For the third equality we used the fact that the relation $\psi_{M}(g h)=\psi_{M}(g)+\psi_{M}(h)$ holds when $g, h$ and $g h$ are all in $N$. For the last equality we used the relation $\psi_{M}(h)^{g^{-1}}$, which also holds when $g, h$ and $g h$ are all in $N$.

We now identify $G$ and $\Gamma$ using the above isomorphism.

Proposition C.3.20. The closure of $\Gamma$ in $W$ is isomorphic to the branch completion of $\Gamma$.

Proof. In the group $\Gamma$, a fundamental system of neighbourhoods of 1 is $\left\{X^{n} * \Gamma^{\prime} \mid\right.$ $n \geq 0\}$ for the branch topology, and $\left\{N_{n} \mid n \geq 0\right\}$ for the subspace topology in $W$. Here $N_{n}$ denotes the kernel of the canonical map $W \rightarrow W_{n}$, intersected with $\Gamma$. We need to show that these two filters are cofinal.

In fact we have the equality $N_{n}=X^{n} * \Gamma^{\prime}$ for all $n \geq 0$. Indeed, notice that we have the isomorphism $\Gamma /\left(X^{n} * \Gamma^{\prime}\right) \simeq\left(\operatorname{Stab}_{\Gamma}(n) /\left(X^{n} * \Gamma^{\prime}\right)\right) \rtimes\left(\Gamma / \operatorname{Stab}_{\Gamma}(n)\right)$, and $\operatorname{Stab}_{\Gamma}(n) /\left(X^{n} * \Gamma^{\prime}\right)$ is a subgroup of $\left(\Gamma / \Gamma^{\prime}\right)^{X^{n}} \simeq A\left[X^{n}\right]$. Thus $\Gamma \rightarrow \Gamma /\left(X^{n} * \Gamma^{\prime}\right)$ and $\Gamma \rightarrow W_{n}$ are in fact one and the same map. Hence their kernels coincide.

Further, we consider the ring $R$ of continuous functions $W \rightarrow \mathbb{Z} / 2 \mathbb{Z}$. In particular we define the following elements of $R$. Consider a subset $S \subseteq \operatorname{Sym}(X)$. We define $\chi_{S}: W \rightarrow \mathbb{Z} / 2 \mathbb{Z}$ as the composition of $W \rightarrow \operatorname{Aut} X^{*} \rightarrow \operatorname{Sym}(X)$ with the characteristic function $\operatorname{Sym}(X) \rightarrow \mathbb{Z} / 2 \mathbb{Z}$ of $S$. For example if $S=$ $\{(1,2),(2,3),(3,1)\}$ then $\chi_{S}$ is the homomorphism $[\sigma]: W \rightarrow \mathbb{Z} / 2 \mathbb{Z}$ which gives the signature of the permutation on top of the tree. We also define the function $[a]: A\left[\left[X^{\omega}\right]\right] \rightarrow \mathbb{Z} / 2 \mathbb{Z}$ as the composition $A\left[\left[X^{\omega}\right]\right] \rightarrow A\left[X^{0}\right] \simeq A$ with the functional $[a]: A \rightarrow \mathbb{Z} / 2 \mathbb{Z}$ which takes the value 1 on $\bar{a}$ and 0 on $\bar{b}$ and $\bar{c}$. We extend this function to $W$ by $[a](m, g)=[a](m)$. We define similarly $[b]$ and $[c]$. Next we define the operation $w * \cdot R \rightarrow R$ by $(w * f)(m, g)=f(m @ w, g @ w)$ for all $w \in X^{*}$ (where $m @ w$ is the coefficient of $m$ with index $w$ in the canonical decomposition $\left.A\left[\left[X^{\omega}\right]\right] \simeq \bigoplus_{w \in X^{n}} A\left[\left[X^{\omega}\right]\right]\right)$. We extend this definition by linearity on the left factor to obtain an operation $*:(\mathbb{Z} / 2 \mathbb{Z})\left[X^{*}\right] \otimes R \rightarrow R$. 
Now we define some specific elements of $R$.

$$
\begin{aligned}
P_{1} & =\chi_{\operatorname{Sym}(X) \backslash \operatorname{Stab}(1)}+(2+3) *([b]+[c]) \\
& =\chi_{\operatorname{Sym}(X) \backslash \operatorname{Stab}(1)}+2 *[b]+2 *[c]+3 *[b]+3 *[c], \\
Q_{1,2} & =\chi_{(1,2) \cdot \operatorname{Stab}(1)}+(1+3) *([b]+[c]) \\
& =\chi_{(1,2) \cdot \operatorname{Stab}(1)}+1 *[b]+1 *[c]+3 *[b]+3 *[c], \\
Q_{1,3} & =\chi_{(1,3) \cdot \operatorname{Stab}(1)}+(2+1) *([b]+[c]) \\
& =\chi_{(1,3) \cdot \operatorname{Stab}(1)}+2 *[b]+2 *[c]+1 *[b]+1 *[c],
\end{aligned}
$$

and we define similarly $P_{2}, P_{3}, Q_{2,1}, Q_{2,3}, Q_{3,1}, Q_{3,2}$. One can check that $Q_{1,2}+$ $Q_{1,3}=P_{1}$ (and similarly for $\left.P_{2}, P_{3}\right)$. We define $\Sigma=[\sigma]+[a]+[b]+[c]$. Then one has $(\varnothing+1+2+3) * \Sigma=(\varnothing+1+2+3) *[\sigma]$ because $(\varnothing+1+2+3) *[a]=[a]$ and similarly for $b, c$.

A subset $S \subseteq W$ is called self-similar if $s @ w \in S$ for all $s \in S, w \in X^{*}$. As one does in algebraic geometry, we write $\mathcal{V}(S)$ for the set of elements $g$ of $W$ so that $P(g)=0$ for all $P \in S$. Define

$$
\widetilde{\Gamma}=\mathcal{V}\left(X^{*} *\left\{\Sigma, P_{1}, P_{2}, P_{3}, Q_{1,2}, Q_{2,3}, Q_{3,1}\right\}\right) .
$$

We prove:

Theorem C.3.21. The group $\widetilde{\Gamma}$ is the branch completion of $\Gamma$.

Note that, for now, it is not even clear that $\widetilde{\Gamma}$ is a group. We prove Theorem C.3.21 in the following lemmata.

Lemma C.3.22. The set $\widetilde{\Gamma}$ is a closed self-similar subgroup of $W$. Moreover, $\Gamma$ is a subgroup of $\widetilde{\Gamma}$.

Proof. Obviously, $\widetilde{\Gamma}$ is self-similar and closed. We need to prove it is a group. Using the self-similarity, it is enough to prove that the projection of the set $\mathcal{V}\left(\left\{\Sigma, P_{1}, P_{2}, P_{3}, Q_{1,2}, Q_{2,3}, Q_{3,1}\right\}\right)$ into $W_{1}$ is a subgroup of $W_{1}$. This is can be done by hand or using a computer, but is in any case straightforward.

Now $\Gamma$ is clearly a subgroup of $\widetilde{\Gamma}$ because $a, b$ and $c$ are in $\widetilde{\Gamma}$.

Remark C.3.23. One can check the relations $P_{1}+P_{2}+P_{3}=Q_{1,2}+Q_{2,1}=$ $Q_{2,3}+Q_{3,2}=Q_{3,1}+Q_{1,3}$. Thus one has $\widetilde{\Gamma}=\mathcal{V}\left(X^{*} *\left\{\Sigma, Q_{1,2}, Q_{1,3}, Q_{2,1}, Q_{2,3}\right\}\right)$, and one can check that $X^{*} *\left\{\Sigma, Q_{1,2}, Q_{1,3}, Q_{2,1}, Q_{2,3}\right\}$ is a minimal generating set for the ideal $I \subset R$ of functions vanishing on $\widetilde{\Gamma}$.

Lemma C.3.24. Let $H$ be the projection of $\widetilde{\Gamma}$ to Aut $X^{*}$. Then the group $\operatorname{Stab}_{H}(n) / \operatorname{Stab}_{H}(n+1)$ has 6 elements when $n=0$, and $2^{2 \cdot 3^{n-1}} \cdot 3^{3^{n}}$ elements for all $n \geq 1$. Moreover, the same holds for $\operatorname{Stab}_{\Gamma}(n) / \operatorname{Stab}_{\Gamma}(n+1)$.

Proof. We first prove that $\operatorname{Stab}_{H}(n) / \operatorname{Stab}_{H}(n+1)$ has at most $2^{2 \cdot 3^{n-1}} \cdot 3^{3^{n}}$ elements for all $n \geq 1$. By definition of $\widetilde{\Gamma}$, the function $(\varnothing+1+2+3) * \Sigma=$ $(\varnothing+1+2+3) *[\sigma]$ must vanish on $\widetilde{\Gamma}$. We set $R=(1+2+3) *[\sigma]$. For every 


\section{C.3. Examples}

element $g \in \widetilde{\Gamma}$ we must have $(w *([\sigma]+R))(g)=0$ for all $w \in X^{*}$. Thus in particular for all $n \geq 1$ and for every element $h$ of $\operatorname{Stab}_{H}(n)$ we have $(w * R)(h)=0$ for all $w \in X^{n-1}$. This implies that $\operatorname{Stab}_{H}(n) / \operatorname{Stab}_{H}(n+1)$ is a subgroup of index at least $2^{3^{n-1}}$ in $\operatorname{Sym}(X)^{X^{n}}$, whence the upper bound.

Now $H$ contains $\Gamma$, therefore it is enough to prove that $\operatorname{Stab}_{\Gamma}(n) / \operatorname{Stab}_{\Gamma}(n+1)$ has at least the desired number of elements. Obviously the group $\Gamma / \operatorname{Stab}_{\Gamma}(1)$ is isomorphic to $\operatorname{Sym}(X)$ and thus has 6 elements. We now consider $n \geq 1$ and show that $\operatorname{Stab}_{\Gamma^{\prime}}(n) / \operatorname{Stab}_{\Gamma^{\prime}}(n+1)$ has at least $2^{2 \cdot 3^{n-1}} \cdot 3^{3^{n}}$ elements.

Notice that $\Gamma^{\prime} / \operatorname{Stab}_{\Gamma^{\prime}}(1)$ is isomorphic to $C_{3}$, generated by the image of $[a, b]=(a b, a, b) \sigma_{123}$ for example. Since $\Gamma^{\prime}$ contains $X^{n} * \Gamma^{\prime}$ for all $n \geq 0$, this also proves that $\operatorname{Stab}_{\Gamma^{\prime}}(n) / \operatorname{Stab}_{\Gamma^{\prime}}(n+1)$ contains a subgroup isomorphic to $C_{3}^{X^{n}}$. Next, we claim $\operatorname{Stab}_{\Gamma^{\prime}}(1) / \operatorname{Stab}_{\Gamma^{\prime}}(2)$ contains a subgroup isomorphic to a Klein four-group $V$. Indeed, a straightforward computation shows

$$
\begin{aligned}
& {[a, b][a, c]=\left((a c, 1, b) \sigma_{13},(1,1,1),(1, b c, a) \sigma_{23}\right),} \\
& {[b, c][b, a]=\left((b, 1, c a) \sigma_{13},(c, b a, 1) \sigma_{12},(1,1,1)\right),}
\end{aligned}
$$

These two elements generate $V$ in the quotient $\operatorname{Stab}_{\Gamma^{\prime}}(1) / \operatorname{Stab}_{\Gamma^{\prime}}(2)$. Using the branching again, this proves that $\operatorname{Stab}_{\Gamma^{\prime}}(n) / \operatorname{Stab}_{\Gamma^{\prime}}(n+1)$ contains a subgroup isomorphic to $V^{X^{n-1}}$ for all $n \geq 1$, which terminates the proof.

Corollary C.3.25. The closure $\bar{\Gamma}$ of $\Gamma$ in the group Aut $X^{*}$ is equal to $H$.

Proof. $\Gamma$ is a subgroup of $H$ and for each $n$, the quotients $H / \operatorname{Stab}_{H}(n)$ and $\Gamma / \operatorname{Stab}_{\Gamma}(n)$ have the same order, hence they are equal. Since $H$ is closed in Aut $X^{*}$, the proposition follows.

Let $\widetilde{\Gamma}_{n}$ be the projection of $\widetilde{\Gamma}$ to $W_{n}$ and let $\widetilde{M}_{n}$ be the kernel of the projection $\widetilde{\Gamma}_{n} \rightarrow$ Aut $X^{n}$. In the same way we write $\Gamma_{n}$ for the projection of $\Gamma$ to $W_{n}$, and we let $M_{n}$ be the kernel of the projection $\Gamma_{n} \rightarrow$ Aut $X^{n}$. The groups $\widetilde{M}_{n}$ and $M_{n}$ are subgroups of $A\left[X^{n}\right]$, and thus are elementary Abelian 2-groups of rank at most $3^{n+1}$.

Lemma C.3.26. (i) The groups $\widetilde{M}_{n}$ and $M_{n}$ have rank 3 when $n=0$, and rank $2 \cdot 3^{n-1}+2$ for all $n \geq 1$.

(ii) The intersection of $M_{n}$ with $\left(\Gamma_{n}\right)^{\prime}$ has rank 0 when $n=0$, and rank $2 \cdot 3^{n-1}$ for all $n \geq 1$.

Proof. We first prove that $2 \cdot 3^{n-1}+2$ is an upper bound for the rank of $\widetilde{M}_{n}$ with $n \geq 1$. The functions in $\left\{w * R\left|w \in X^{*},\right| w \mid \leq n-1, R \in\left\{Q_{1,2}, Q_{1,3}, Q_{2,1}, Q_{2,3}\right\}\right\}$ and $\left\{w * \Sigma \mid w \in X^{n-1}\right\}$ give $2\left(3^{n}-1\right)+3^{n-1}$ homogeneous linear equations on $\operatorname{ker}\left(W_{n} \rightarrow\right.$ Aut $\left.X^{n}\right) \simeq A^{X^{n}}$. Moreover, it is straightforward to check that they are all linearly independent. Thus $\widetilde{M}_{n}$ has rank at most $3^{n+1}-\left(2\left(3^{n}-1\right)+3^{n-1}\right)=$ $2 \cdot 3^{n-1}+2$, as we claimed.

Since $\Gamma$ is a subgroup of $\widetilde{\Gamma}$, we have the inclusion $M_{n} \subseteq \widetilde{M}_{n}$. Obviously $M_{0} \simeq \Gamma_{0}$ is isomorphic to $A$, which is of rank 3. Also, $\Gamma_{0}^{\prime}$ is the trivial group. 
We now consider $n \geq 1$. On the elements of the group $M_{n} \cap \Gamma_{n}^{\prime}$, the functions $[a],[b]$ and $[c]$ must vanish. This gives only two more homogeneous linear equations on $M_{n}$, because $[a]+[b]+[c]=\Sigma$ when restricted to the group $M_{n}$. This shows that $M_{n} \cap \Gamma_{n}^{\prime}$ has rank at most $2 \cdot 3^{n-1}$. Consider the elements

$$
[a, b][b, c]^{a}=(1, a b c, b a c), \quad[b, c][c, a]^{b}=(c b a, 1, b c a), \quad[c, a][a, b]^{c}=(c a b, a c b, 1) .
$$

Their images in $M_{1} \cap \Gamma_{1}^{\prime}$ are the non-trivial elements of a Klein four-group $V$. Since $\Gamma^{\prime}$ contains $X^{n} * \Gamma^{\prime}$ for all $n \geq 0$, we see that $M_{n} \cap \Gamma_{n}^{\prime}$ contains $V^{X^{n-1}}$, which has $\operatorname{rank} 2 \cdot 3^{n-1}$.

Finally, define

$$
x_{a}=b^{a} c=(a, a, b c), \quad x_{b}=c^{b} a=(c a, b, b), \quad x_{c}=a^{c} b=(c, a b, c) .
$$

The images of these element in $M_{1}$ generate a Klein four-group $V$. But none of these elements belong to $\Gamma^{\prime}$, and therefore this group has trivial intersection with $M_{1} \cap \Gamma_{1}^{\prime}$. Moreover, we have

$$
x_{a} x_{b} x_{c} \equiv x_{a}^{2} \equiv x_{b}^{2} \equiv x_{c}^{2} \equiv 1 \quad\left(\bmod \Gamma^{\prime}\right),
$$

and

$$
x_{a}^{[c, a]} \equiv(c b, a, a) \equiv\left(x_{a}, a, a\right) \quad\left(\bmod \Gamma^{\prime}\right) .
$$

Thus we have the relation

$$
x_{c} \equiv x_{a} x_{b} \equiv\left(x_{a} x_{b}, a b, a b\right) \equiv\left(x_{c}, x_{c}, x_{c}\right) \quad\left(\bmod \Gamma^{\prime}\right),
$$

and similarly $x_{a} \equiv\left(x_{a}, x_{a}, x_{a}\right)$ and $x_{b} \equiv\left(x_{b}, x_{b}, x_{b}\right)$. We conclude that $M_{n}$ contains a Klein group generated by the image of $\prod_{w \in X^{n}} w * x_{s}$, with $s \in\{a, b, c\}$. And this group has trivial intersection with $M_{n} \cap \Gamma_{n}^{\prime}$.

Corollary C.3.27. The group $\Gamma$ is dense in $\widetilde{\Gamma}$.

Proof. $\Gamma$ is a subgroup of $\widetilde{\Gamma}$ and for each $n$, the quotients $\widetilde{\Gamma}_{n}$ and $\Gamma_{n}$ have the same order, hence they are equal. Since $\widetilde{\Gamma}$ is the inverse limit of its quotients $\widetilde{\Gamma}_{n}$, the proposition follows.

Proof of Theorem C.3.21. The group $\Gamma$ embeds densely in $\widetilde{\Gamma}$, which is a closed subgroup of $W$. Proposition C.3.20 concludes.

Proposition C.3.28. The branch kernel $\operatorname{ker}(\widetilde{\Gamma} \rightarrow \bar{\Gamma})$ is a Klein group of order 4.

Proof. Reading carefully the proof of Lemma C.3.26, one can see that Equation (C.3.1) and the corresponding ones for $x_{a}$ and $x_{b}$ are the key for the computation of the inverse limit

$$
\varliminf_{m \geq 0}\left(\bigcap_{n \geq 0} \operatorname{Stab}_{\Gamma}(n)\left(X^{m} * \Gamma^{\prime}\right) /\left(X^{m} * \Gamma^{\prime}\right)\right)=\underset{n \geq 0}{\lim _{n \geq 0}} \widetilde{\operatorname{Stab}_{\Gamma}(n)} .
$$




\section{C.3. Examples}

Indeed, write $\bar{x}_{a}=\bar{b} \bar{c} \in A$, and similarly $\bar{x}_{b}=\bar{a} \bar{c}$ and $\bar{x}_{c}=\bar{a} \bar{b}$. Then the group $\bigcap_{n>0} \operatorname{Stab}_{\Gamma}(n)\left(X^{m} * \Gamma^{\prime}\right) /\left(X^{m} * \Gamma^{\prime}\right)$ can be seen as a subgroup of $A\left[X^{n}\right]$, namely the Klein group whose non-trivial elements are $X^{m} * \bar{x}_{a}, X^{m} * \bar{x}_{b}$ and $X^{m} * \bar{x}_{c}$ (with the notation $X^{m} * s=\sum_{w \in X^{m}} w * s$ ). The map $X^{m} \rightarrow X^{k}$ sends $X^{m} * s \rightarrow X^{k} * s$ for all $s \in A$ and $m \geq k$, and therefore the rigid kernel of $\Gamma$ is the subgroup of $A\left[\left[X^{\omega}\right]\right]$ generated by $X^{\omega} * \bar{x}_{a}, X^{\omega} * \bar{x}_{b}$ and $X^{\omega} * \bar{x}_{c}$ : a Klein group of order 4 .

\section{The Congruence Kernel of $\Gamma$}

We put together the results of the last two sections to prove just a bit more than Theorem C.3.11:

Theorem C.3.29. The congruence kernel of $\Gamma$ is an extension of a Klein group $V$ by $\widehat{\mathbb{Z}}^{3}\left[\left[X^{\omega}\right]\right]$. The action of $V$ is diagonal. Each non-trivial element of $V$ acts as a half-turn along a coordinate axis on $\widehat{\mathbb{Z}}^{3}$.

Proof. From Equation (C.3.1), it is clear that the action of $V$ is diagonal on each level. Comparing the proof of Proposition C.3.18 and of Lemma C.3.26, we see that it is sufficient to look at the action of $b c$ on $d, e, f$, up to cyclic permutation of $a, b, c$, and $d, e, f$. One has

$$
d^{b c} \simeq d^{-1}, \quad \quad e^{b c}=e, \quad f^{b c} \simeq f^{-1},
$$

where the $\simeq$ sign indicates that the equality holds $\bmod \Gamma^{\prime \prime}$, and up to the $C_{3}$ factor inside $\Gamma^{\prime} / \Gamma^{\prime \prime}$. Since this $C_{3}$ disappears in the inverse limit defining the branch kernel, it is irrelevant here.

Theorem C.3.30. The congruence kernel of $\Gamma$ is torsion-free; in particular, the extension $\widehat{\mathbb{Z}}^{3}\left[\left[X^{\omega}\right]\right] \cdot V$ is not split.

Proof. By Theorem C.3.29, $V$ acts on the quotient $\widehat{\mathbb{Z}}^{3}\left[X^{0}\right]=\widehat{\mathbb{Z}}^{3}$ generated by $d, e, f$. We prove that the corresponding extension

$$
1 \rightarrow \widehat{\mathbb{Z}}^{3} \rightarrow K \rightarrow V \rightarrow 1
$$

is torsion-free; the claim follows.

Using cyclic permutation of $a, b, c$, it is enough to prove that any lift of $b c \in V$ to $K$ has infinite order. Such a lift can be written as $x=d^{\alpha} e^{\beta} f^{\gamma}(b c)$ for some $\alpha, \beta, \gamma \in \widehat{\mathbb{Z}}$. We compute

$$
x^{2}=\left(d^{\alpha} e^{\beta} f^{\gamma}(b c)\right)^{2}=e^{2 \beta}(b c)^{2}=e^{2 \beta+1} .
$$

Since the equation $2 \beta+1=0$ has no solution in $\widehat{\mathbb{Z}}$, we see that $x^{2} \in \widehat{\mathbb{Z}}^{3}$ is not trivial, and therefore $x$ has infinite order. 


\title{
Appendix D
}

\section{The Twisted Twin of the Grigorchuk Group ${ }^{1}$}

\begin{abstract}
We study a twisted version of Grigorchuk's first group, and stress its similarities and differences to its model.

In particular, we show that it admits a finite endomorphic presentation, has infinite-rank multiplier, and does not have the congruence property.
\end{abstract}

\section{D.1 Introduction}

The first Grigorchuk group $\Gamma$ appeared in [Gri80] in the early 1980's, as an example of a finitely generated, infinite, torsion group. It was soon shown to be just-infinite, and to have intermediate word growth [Gri83], answering Milnor's question [Mil68]. To this date it remains the fundamental example of such a group, and a rich source of examples throughout group theory.

The profinite completion $\bar{\Gamma}$ of $\Gamma$ was quickly seen to be an interesting pro-2 group, deserving further study [Gri00]. It is then natural to ask which properties of $\Gamma$ are actually associated with its completion, and which depend on the precise nature of $\Gamma$ (sitting as a specific, dense subgroup of $\bar{\Gamma}$ ).

The usual description of $\Gamma$ is as a "group generated by automata". As such, it acts on a binary rooted tree, and its elements are described by finite-state transducer automata. One may close $\Gamma$ using the topology of the automorphism group of the binary rooted tree, and this topology coincides with the profinite topology on $\Gamma$. Sunic and Grigorchuk asked in 2007, in Cardiff, whether the closure $\bar{\Gamma}$ of $\Gamma$ contained other finite-state transducer automata, and in particular other with only one non-trivial cycle (so-called "bounded automata").

In answering that question, the second author discovered a "twisted" version $G$ of $\Gamma$, and the purpose of the present paper is to study some of its algebraic properties. In summary, $G$ also acts on the infinite binary tree, and shares many properties with $\Gamma$ :

- $G$ is torsion, just-infinite, and has the same closure as $\Gamma$ in the group of automorphisms of the tree;

\footnotetext{
${ }^{1}$ Joint work with Laurent Bartholdi
} 
- $G$ has a finite endomorphic presentation

$$
\left.G=\langle a, \beta, \gamma, \delta| a^{2}, \beta^{2}, \gamma^{2}, \delta^{2}, \varphi^{n}(R) \text { for all } n \geq 0\right\rangle,
$$

where $R=\left\{\left[\delta^{a}, \delta\right],\left[\delta, \gamma^{a} \beta\right],\left[\delta,\left(\gamma^{a} \beta\right)^{\gamma}\right],\left[\gamma^{a} \beta, \gamma \beta^{a}\right]\right\}$ and $\varphi$ is the endomorphism of the free group on $a, \beta, \gamma, \delta$ induced by

$$
a \mapsto \gamma^{a}, \quad \beta \mapsto \delta, \quad \gamma \mapsto \beta^{a}, \quad \delta \mapsto \gamma,
$$

see Theorem D.4.1. Moreover, its relators are independent in $H_{2}(G, \mathbb{Z})$ which, qua $(\mathbb{Z} / 2 \mathbb{Z})[\varphi]$-module, is freely generated by $R$, see Theorem D.8.9;

- $G$ has bounded width; more precisely, if $\left(\gamma_{n}\right)$ denote its lower central series, then $\gamma_{n} / \gamma_{n+1}$ is an elementary Abelian 2-group of rank 2 if $\frac{3}{4} 2^{i}+1 \leq n \leq 2^{i}$ for some $i$, and of rank 4 otherwise, see Corollary D.7.5;

- $G$ and $\Gamma$ are not isomorphic; $\Gamma$ does not have the congruence property, and more precisely its congruence kernel is isomorphic to $(\mathbb{Z} / 4 \mathbb{Z})\left[\left[\{0,1\}^{\omega}\right]\right]$ as a profinite $\bar{G}$-module.

Since $G$ is a group of "bounded" automorphisms of the binary tree (see the next reference for a definition), it follows from [BKN08] that $G$ is amenable. We were unable to estimate the word growth of $G$, however.

This paper is related to [BSZ09] which studies more systematically the congruence problem for groups generated by automata.

Another purpose of this paper is to assemble, in a systematic manner, the various tools and techniques used to study a new example of group generated by automata. We believe that the very computational nature of these groups lends itself to computer calculations, and we make liberal use of GAP [GAP08] to complete several steps.

We use the Knuth-Bendix procedure implemented in the package KBMAG [KBMAG09] to improve a presentation of our group. Many proofs reduce to computations in finite permutation groups; they are easily performed in GAP. Some other proofs are more involved, often relying on an induction process. The induction basis boils down to verifications in nilpotent quotients of L-presented groups and happily, such quotients are now efficiently implemented in the package NQL [NQL09], see [BEH08].

We insist however that these computations could very well have been done, and written down, by hand, at the cost of trebling the length of this paper; and that the statements of this paper are true and not empirical facts.

The methods presented here can all be applied with only slight modifications to the Grigorchuk group, or to other groups generated by automata, yet to come under the spotlight. We therefore include the GAP command sequences we have used in an appendix.

\section{D.2 Basic Definitions and Notation}

We introduce the necessary vocabulary describing groups acting on rooted trees. The main notions are summarized in Table D.1. 
We consider the alphabet $X=\{0,1\}$ and we identify the free monoid $X^{*}$ over $X$ with the vertices of an infinite binary tree. We let Aut $X^{*}$ denote the automorphism group of the tree $X^{*}$.

Let $v$ be a vertex of $X^{*}$. The stabilizer of $v$ is the group $\operatorname{Stab}(v)$ of elements of Aut $X^{*}$ which fix $v$. The $n$-th level stabilizer, written $\operatorname{Stab}(n)$, is the subgroup of Aut $X^{*}$ consisting of the elements that fix all the vertices of the $n$-th level. It is a normal subgroup of Aut $X^{*}$. The quotient Aut $X^{n}=\operatorname{Aut} X^{*} / \operatorname{Stab}(n)$ is a finite 2-group isomorphic to the $n$-th fold iterated wreath product of $\operatorname{Sym}(X)$, a cyclic group of order 2. Moreover, the group Aut $X^{*}$ is the projective limit of the projective system Aut $X^{n}$, and hence Aut $X^{*}$ is a profinite group. A basis of neighbourhoods of the identity is given by the collection $\{\operatorname{Stab}(n): n \geq 0\}$.

For $n \geq 0$, we define the map

$$
\begin{aligned}
\psi_{n}: \text { Aut } X^{*} & \rightarrow \text { Aut } X^{*} \imath \text { Aut } X^{n} \\
g & \mapsto\left(g @ v \mid v \in X^{n}\right) \sigma .
\end{aligned}
$$

We shall often write $\psi$ for $\psi_{1}$. The above defines the symbol $g @ v$. Namely, $g @ v$ is the projection of $g$ onto the $v$-th coordinate under $\psi_{n}$, where $n$ is the length of $v$. A subset $S \subseteq$ Aut $X^{*}$ is called self-similar if $S @ v$ is contained in $S$ for all $v \in X^{*}$. For a vertex $v \in X^{*}$ and an automorphism $g \in$ Aut $X^{*}$, we let $v * g$ denote the automorphism satisfying

$$
\psi_{|v|}(v * g)=(1, \ldots, 1, g, 1, \ldots, 1),
$$

so that $(v * g) @ v=g$.

Consider a group $G \leq$ Aut $X^{*}$. The group $G$ is level-transitive if the action of $G$ is transitive on each level $X^{n}$. The group $G$ is recurrent if $\operatorname{Stab}_{G}(v) @ v=G$ for all $v \in X^{*}$. A branching subgroup is a subgroup $K \leq G$ so that $X^{n} * K=$ $\prod_{v \in X^{n}} v * K$ is a subgroup of $K$ for all $n \geq 0$. The group $G$ is weakly regular branch if it has a non-trivial branching subgroup. The group $G$ is regular branch if it is weakly regular branch and $X^{n} * K$ has finite index in $G$ for all $n \geq 0$. In such a situation we say that $G$ is regular branch over $K$.

\section{D.3 Definition of the Group}

We define recursively the following elements of Aut $X^{*}$ :

$$
a=\sigma, \quad \psi(\beta)=(\gamma, a), \quad \psi(\gamma)=(a, \delta), \quad \psi(\delta)=(1, \beta),
$$

where $\sigma$ is the automorphism which permutes the two maximal subtrees. We let $G$ be the group generated by $a, \beta, \gamma, \delta$. We shall call this group the twisted twin of the Grigorchuk group. The motivation for this terminology is that the automaton defining $G$ is very similar to the one defining the Grigorchuk group, and moreover both groups have identical closure in Aut $X^{*}$ (see Proposition A.2.1). However, $G$ is not isomorphic to the Grigorchuk group as we shall see.

Let $N$ be the generating set $\{1, a, \beta, \gamma, \delta\}$ of $G$. Clearly $N$ is self-similar, and therefore so is $G$. Next, $G$ acts transitively on $X$, and it is recurrent on the 


\section{D.3. Definition of the Group}

\begin{tabular}{|c|c|c|c|}
\hline symbol & \multicolumn{2}{|l|}{ name } & definition \\
\hline $\begin{array}{c}X^{*} \\
X^{n} \\
\psi_{n} \\
g @ v\end{array}$ & \multicolumn{2}{|c|}{$\begin{array}{l}\text { infinite binary tree } \\
n \text {-th level of } X^{*} \\
\text { wreath decomposition } \\
\text { state of } g \text { at } v\end{array}$} & $\begin{array}{l}\text { canonical map Aut } X^{*} \rightarrow \operatorname{Aut} X^{*} \imath \text { Aut } X^{n} \\
\text { projection of } g \text { on the } v \text {-th coordinate in } \\
\psi_{n}\left(\text { Aut } X^{*}\right) \\
\text { element of } \operatorname{Rist}(v) \text { with }(v * g) @ v=g \\
\text { subgroup with } v * K \leq K \text { for all } v \in X^{*} \\
\text { product of } v * K \text { for all } v \in X^{n}\end{array}$ \\
\hline $\operatorname{Stab}_{G}(v)$ & \multicolumn{2}{|c|}{$\begin{array}{l}\text { stabilizer of } v \\
n \text {-th level stabilizer }\end{array}$} & $\begin{array}{l}\text { subgroup of } G \text { consisting of the elements } \\
\text { which fix } v \\
\text { intersection of } \operatorname{Stab}_{G}(v) \text { with } v \text { ranging } \\
\text { over } X^{n}\end{array}$ \\
\hline \multicolumn{2}{|c|}{$\begin{array}{l}\text { level-transitive } \\
\text { self-similar } \\
\text { recurrent } \\
\text { weakly regular branch } \\
\text { regular branch }\end{array}$} & \multicolumn{2}{|c|}{$\begin{array}{l}\text { the action of } G \text { is transitive on each level } \\
\text { the image of } G \rightarrow \operatorname{Aut} X^{*} \imath \operatorname{Sym}(X) \text { is contained in } \\
G \prec \operatorname{Sym}(X) \\
\operatorname{Stab}_{G}(v) @ v=G \text { for all } v \in X^{*} \\
G \text { contains a non-trivial branching subgroup } \\
\text { weakly regular branch, and } X^{n} * K \text { has finite index in } \\
G \text { for all } n \geq 0\end{array}$} \\
\hline
\end{tabular}

Table D.1: Symbols, subgroups, and main properties of groups acting on rooted trees.

first level: $\operatorname{Stab}_{G}(x) @ x=G$ for all $x \in X$. Therefore $G$ is level-transitive and recurrent. In particular, this implies that $G$ is infinite.

Define the group $K \leq G$ by

$$
K=\langle[a, \beta],[\beta, \gamma],[\beta, \delta],[\gamma, \delta], \beta \gamma \delta\rangle^{G}
$$

where the exponent stands for normal closure in $G$.

Proposition D.3.1. The group $G$ is regular branch over $K$. Moreover, $K$ contains $\operatorname{Stab}_{G}(3)$.

Proof. Write $B=\langle\beta, \gamma \delta\rangle^{G}$. The group $G / B$ is generated by the images of $a$ and $\delta$, and therefore has order at most $|\langle a, \delta\rangle|=8$.

The group $B / K$ is generated by the image of $\beta$, hence it is of order at most 2. Therefore $K$ is of index at most 16 in $G$. A direct computation shows that $K / \operatorname{Stab}_{K}(3)$ has index 16 in $G / \operatorname{Stab}_{G}(3)$. This proves that $K$ has index 2 in $B$, that $B$ has index 8 in $G$, and that $K$ contains $\operatorname{Stab}_{G}(3)$.

We now prove that $X * K$ is contained in $K$. For this we simply show that $1 * g$ is contained in $K$ for all $g \in\{[a, \beta],[\beta, \gamma],[\beta, \delta],[\gamma, \delta], \beta \gamma \delta\}$ :

$$
\begin{array}{rlrl}
{[\beta, \delta]} & =1 *[a, \beta], & {[\delta, \beta] \cdot[\delta,[a, \beta]]^{\beta}} & =1 *[\beta, \gamma], \\
{[\delta, \gamma]} & =1 *[\beta, \delta], & {[[a, \beta], \gamma]^{\beta} \cdot[\beta, \gamma]} & =1 *[\gamma, \delta], \\
([a, \beta] \beta \gamma \delta)^{\delta}=\delta \beta^{a} \gamma & =1 * \beta \gamma \delta . &
\end{array}
$$


The last thing to prove is that $X^{n} * K$ has finite index in $K$. Since $G$ is selfsimilar, $\operatorname{Stab}_{G}(n+3)$ is a subgroup of $X^{n} * \operatorname{Stab}_{G}(3)$. Now $X^{n} * K$ contains $X^{n} * \operatorname{Stab}_{G}(3)$, and therefore it also contains $\operatorname{Stab}_{G}(n+3)$, which has finite index in $G$.

Remark D.3.2. Further computations show that the Abelianization of $K$ is $C_{2} \times$ $\left(C_{4}\right)^{3} \times C_{8}$ and that $K /(X * K)$ is a cyclic group of order 4 .

\section{D.4 A Presentation}

Recall that an (invariant) L-presentation is an expression of the form

$$
\langle X|\Phi| R\rangle
$$

where $X$ is a set, $\Phi$ is a collection of endomorphisms of the free group $F_{X}$ with basis $X$, and $R$ is a set of elements of $F_{X}$. The group defined by this L-presentation is then $\langle X \mid\{\phi(r)\}\rangle$ where $\phi$ ranges over the monoid generated by $\Phi$, and $r$ ranges over $R$. Obviously, each endomorphism in $\Phi$ induces an endomorphism of the L-presented group.

L-presentations were introduced in [Bar03]; a fundamental example was Lysionok's presentation of the Grigorchuk group [Lys85].

The main result of this section is the following

Theorem D.4.1. The group $G$ admits the L-presentation

$$
G=\left\langle a, \beta, \gamma, \delta|\tilde{\varphi}| a^{2}, \beta^{2}, \gamma^{2}, \delta^{2},\left[\delta^{a}, \delta\right],\left[\delta, \gamma^{a} \beta\right],\left[\delta,\left(\gamma^{a} \beta\right)^{\gamma}\right],\left[\gamma^{a} \beta, \gamma \beta^{a}\right]\right\rangle,
$$

where $\tilde{\varphi}$ is the endomorphism of the free group on $a, \beta, \gamma, \delta$ induced by

$$
a \mapsto \gamma^{a}, \quad \beta \mapsto \delta, \quad \gamma \mapsto \beta^{a}, \quad \delta \mapsto \gamma .
$$

Let $\varphi$ be the following map, defined on the generators of $G$ :

$$
\begin{array}{rlrl}
a & \mapsto \gamma^{a}=\psi^{-1}(\delta, a), & \beta \mapsto \delta=\psi^{-1}(1, \beta), \\
\gamma \mapsto \beta^{a}=\psi^{-1}(a, \gamma), & \delta \mapsto \gamma=\psi^{-1}(a, \delta) .
\end{array}
$$

Proposition D.4.2. The map $\varphi$ extends to an endomorphism of $G$.

Proof. From the above it is enough to prove that the map defined by

$$
a \mapsto \delta, \quad \beta \mapsto 1, \quad \gamma \mapsto a, \quad \delta \mapsto a
$$

extends to a homomorphism $G \rightarrow G$. The group generated by $a$ and $\delta$ is a dihedral group of order 8 , and one can check that the map above factors through $G \rightarrow G / \operatorname{Stab}_{G}(3)$.

It is immediate that the restriction of $\varphi$ to $K$ sends $x$ to $\psi^{-1}(1, x)$. We shall now prove Theorem D.4.1 and then some results which follow from this theorem. 


\section{D.4. A Presentation}

Definition D.4.3. A self-similar group $G \leq$ Aut $X^{*}$ is contracting if there is a finite set $N \subseteq G$ such that for every $g \in G$ there is an integer $n$ such that $g @ w \in N$ for all $w \in X^{*}$ of length at least $n$. The minimal set $N$ with this property is the nucleus of $G$.

Lemma D.4.4. The group $G$ is contracting with nucleus $N=\{1, a, \beta, \gamma, \delta\}$.

Proof. There are several ways of proving that $G$ is contracting. The most direct one is to notice that $N$ generates $G$; and that, if $g$ is in $N^{2}$, then $g @ w$ is in $N$ for all $w$ of length at least 3 .

It is then easy to prove that $N$ is the nucleus of $G$, by induction on the length of the elements (see [Nek05] where this is done in detail, and in greater generality).

We recall the general strategy in obtaining presentations by generators and relations for self-similar groups; for more details see [Bar03] or [Sid87].

The only relations of length $\leq 3$ among elements of $N$ are $a^{2}=\beta^{2}=\gamma^{2}=$ $\delta^{2}=1$. We thus consider the group $F=\left\langle a, \beta, \gamma, \delta \mid a^{2}, \beta^{2}, \gamma^{2}, \delta^{2}\right\rangle$. The decomposition map $\psi: G \rightarrow G$ ¿ $\operatorname{Sym}(X)$ restricts to a map $N \rightarrow N^{X} \times \operatorname{Sym}(X)$, which induces a homomorphism $\bar{\psi}: F \rightarrow F$ ¿ $\operatorname{Sym}(X)$. Thanks to the relations of length $\leq 3$ we took in $F$, the recursion $\bar{\psi}$ is also contracting, with nucleus $\{1, a, \beta, \gamma, \delta\}$; see again [Nek05] for details. Set $R_{0}=1 \triangleleft F$ and $R_{n+1}=\psi^{-1}\left(R_{n}^{X}\right)$ for all $n \geq 0$.

Lemma D.4.5. $G=F / \bigcup_{n \geq 0} R_{n}$.

Proof. By our choice of relations in $F$, the decomposition $\bar{\psi}$ is contracting on $F$, with nucleus $\{1, a, \beta, \gamma, \delta\}$. Given $w \in F$ : if $w$ belongs to $R_{n}$ for some $n$, then it is clear that $w$ is trivial in $G$. Conversely, if $w$ is trivial in $G$, there exists $n$ such that all $w$ 's level- $n$ states belong to $N$ and act trivially; so they are all 1 ; so $w$ belongs to $R_{n}$.

Using the Reidemeister-Schreier rewriting method, we can construct a normal generating set for $R_{1}$. Indeed, $\bar{\psi}$ induces an injective map $F / R_{1} \rightarrow F$ 乙 $\operatorname{Sym}(X)$, whose image has infinite index. However, we may chose the infinite dihedral group generated by $a$ and $\delta$ on the first copy of $F$ as a transversal for $\bar{\psi}(F)$ in $F$ 々 $\operatorname{Sym}(X)$. Then we obtain quite explicitly

$$
R_{1}=\left\langle\left[\delta^{a}, \delta^{w}\right],\left[\delta^{a},\left(\gamma \beta^{a}\right)^{w}\right],\left[\left(\gamma \beta^{a}\right)^{a},\left(\gamma \beta^{a}\right)^{w}\right]\right\rangle^{F},
$$

where $w$ ranges over the elements of the infinite dihedral group generated by $\gamma$ and $\gamma^{a}$.

We now consider the homomorphism $\bar{\varphi}: F \rightarrow F$ defined by

$$
a \mapsto \gamma^{a}, \quad \beta \mapsto \delta, \quad \gamma \mapsto \beta^{a}, \quad \delta \mapsto \gamma .
$$

By Proposition D.4.2, for all normal generators $r$ of $R_{1}$ we have $\bar{\psi}(\bar{\varphi}(r))=\left(r^{\prime}, r\right)$ for some relation $r^{\prime} \in\langle a, \delta\rangle$. It happens that $(a \delta)^{4}$ is in $R_{1}$ and that $\langle a, \delta\rangle$ is a dihedral group of order 8 in $G$. Therefore $r^{\prime}$ is in $R_{1}$ and $R_{n}$ is normally generated by $\bigcup_{i<n} \bar{\varphi}^{i}\left(R_{1}\right)$ for all $n>0$. We conclude: 


\section{Proposition D.4.6.}

$$
\left.G=\langle a, \beta, \gamma, \delta| a^{2}, \beta^{2}, \gamma^{2}, \delta^{2}, \tilde{\varphi}^{n}(r) \text { for all } n \geq 0\right\rangle,
$$

where $r$ ranges over the normal generators of $R_{1}$ above.

Proof of Theorem D.4.1. One of the $r$ above is $\left[\delta^{a}, \delta\right]$. Applying $\tilde{\varphi}$ we get $\tilde{\varphi}\left(\left[\delta^{a}, \delta\right]\right) \equiv$ $\left(\gamma \gamma^{a}\right)^{4}=1$. Therefore it is enough to consider 8 values of $w$, for example the following ones:

$$
w \in\left\{1, \gamma, \gamma^{a}, \gamma \gamma^{a}, \gamma^{a} \gamma, \gamma \gamma^{a} \gamma, \gamma^{a} \gamma \gamma^{a}, \gamma \gamma^{a} \gamma \gamma^{a}\right\}
$$

Also, $\tilde{\varphi}(s)$ is clearly a consequence for $s$ in $\left\{a^{2}, \beta^{2}, \gamma^{2}, \delta^{2}\right\}$. Thus we proved the following

$$
G=\left\langle a, \beta, \gamma, \delta|\tilde{\varphi}| a^{2}, \beta^{2}, \gamma^{2}, \delta^{2},\left[\delta^{a}, \delta^{w}\right],\left[\delta^{a},\left(\gamma \beta^{a}\right)^{w}\right],\left[\gamma \beta^{a},\left(\gamma \beta^{a}\right)^{w}\right]\right\rangle,
$$

with $w$ ranging over the set above. Then we use a computer to prove that the normal closure of the relations in the claim together with their iterates under $\tilde{\varphi}$ contains all the relations of the presentation (D.4.1). This is done using GAP, in Lemma D.9.1.

Remark D.4.7. In the same way, one can also recover the usual presentation of the Grigorchuk group (see [Lys85]):

$$
\left\langle a, b, c, d|\phi| a^{2}, b^{2}, c^{2}, d^{2}, b c d,\left[d^{a}, d\right],\left[d^{a}, d^{c^{a} c}\right]\right\rangle
$$

where $\phi$ is given by

$$
a \mapsto c^{a}, \quad b \mapsto d, \quad c \mapsto b, \quad d \mapsto c .
$$

Knowing a presentation (even an infinite one) is very useful in practice. For example, this makes the following computations straightforward.

Proposition D.4.8. The group $K$ contains $\gamma_{3}(G)$.

Proof. Using Theorem D.4.1, we see that $G / K$ is (a quotient of) the group presented by

$$
\left\langle a, \gamma, \delta \mid a^{2}, \gamma^{2}, \delta^{2},[\gamma, \delta],[a, \gamma \delta],\left[\delta, \delta^{a}\right],\left[\gamma, \delta^{a}\right],\left[\gamma, \gamma^{a}\right]\right\rangle
$$

A Todd-Coxeter procedure shows that this group has order 16 and is nilpotent of class 2 .

Define the group

$$
A=\langle a\rangle^{G}
$$

Proposition D.4.9. The group A contains $\gamma_{3}(G)$ and has index 16 in $G$. 


\section{D.5. The Congruence Kernel}

Proof. From Theorem D.4.1 we see that the group $G / A$ admits the presentation

$$
\left\langle\beta, \gamma, \delta \mid \beta^{2}, \gamma^{2}, \delta^{2},[\delta, \gamma \beta],[\gamma, \beta \delta],[\beta, \delta \gamma]\right\rangle .
$$

A Todd-Coxeter procedure shows that this group has order 16 and is nilpotent of class 2 .

The following Proposition is needed in the article [BSZ09].

Proposition D.4.10. The group $[K, G]$ has index 128 in $G$ and is generated by $\gamma_{3}(G)$ and $[a, \beta \gamma \delta]$. Moreover, $[K, G]$ contains $\gamma_{3}(G)$ and the quotient $K /[K, G]$ is isomorphic to $C_{4} \times C_{2}$, where $C_{4}$ is generated by $\beta \gamma \delta$ and $C_{2}$ is generated by $[a, \beta]$.

Proof. Since $K$ contains $\gamma_{3}(G)$, the group $[K, G]$ contains $\gamma_{4}(G)$. Therefore, all computations can be made in $G / \gamma_{4}(G)$, which is a finite 2-group. The rest is routine check using NQL [NQL09].

Let $C$ be the group generated by $[K, G]$ and $[a, \beta][\beta, \gamma]$.

Proposition D.4.11. The group $C$ is normal, of index 64 in $G$. Moreover, $C$ contains $\gamma_{3}(G)$ and the quotient $K / C$ is isomorphic to $C_{4}$, generated by $\beta \gamma \delta$.

Proof. Since $[K, G]$ contains $\gamma_{3}(G)$, all computations can be made in $G / \gamma_{3}(G)$, which is a finite 2-group. The rest is routine check using NQL [NQL09].

\section{D.5 The Congruence Kernel}

\section{D.5.1 A Basis of Neighbourhoods of the Identity in $\widehat{G}$}

Definition D.5.1. Let $X$ be a set and let $\left\{A_{i}\right\}_{i \in I}$ and $\left\{B_{j}\right\}_{j \in J}$ be two filters in $X$. We say $\left\{A_{i}\right\}$ is cofinal to $\left\{B_{j}\right\}$ if for every $j \in J$, there is $i \in I$ such that the inclusion $A_{i} \leq B_{j}$ holds. We say $\left\{A_{i}\right\}$ and $\left\{B_{j}\right\}$ are cofinal if $\left\{A_{i}\right\}$ is cofinal to $\left\{B_{j}\right\}$ and $\left\{B_{j}\right\}$ is cofinal to $\left\{A_{i}\right\}$.

Definition D.5.2. Let $G$ be a subgroup of Aut $X^{*}$. A subgroup $H \leq G$ is called a congruence subgroup if $H$ contains $\operatorname{Stab}_{G}(n)$ for some $n \geq 0$.

Consider the three collections $\mathcal{C}=\left\{\operatorname{Stab}_{G}(n): n \geq 0\right\}, \mathcal{B}=\left\{X^{n} * K\right.$ : $n \geq 0\}$ and $\mathcal{P}=\{N \leq G: N$ is normal of finite index in $G\}$. Taken as basis of neighbourhoods of the identity in $G$, they define the congruence, branch and profinite topology, respectively.

We already know that $\mathcal{C}$ and $\mathcal{B}$ are cofinal by Proposition D.3.1. That is, every $X^{n} * K$ is a congruence subgroup. This implies that the congruence and the branch topologies coincide, and therefore the rigid kernel of $G$ is trivial, in the terminology introduced in [BSZ09].

The completion of $G$ with respect to $\mathcal{C}$ is $\bar{G}=\lim _{\longleftarrow} G / \operatorname{Stab}_{G}(n)$; this group is the closure of $G$ in Aut $X^{*}$. The completion of $G$ with respect to $\mathcal{P}$ is $\widehat{G}=$ $\lim _{\mathcal{P}} G / N$, this is the profinite completion of $G$. 
Recall the group $C$ from Proposition D.4.11. We now prove that the collection $\mathcal{P}$ and the sequence $\left\{X^{n} * C: n \in \mathbb{N}\right\}$ are cofinal. We shall see later that the groups $X^{n} * C$ are not congruence subgroups. Therefore the branch kernel of $G$ is non-trivial.

Proposition D.5.3. Any non-trivial normal subgroup of $G$ contains $X^{n} * C$ for all $n$ big enough.

Corollary D.5.4. $G$ is just-infinite.

The proof of the proposition splits into three lemmas. The following has been extracted from the proof of [Gri00, Theorem 4].

Lemma D.5.5. Let $G \leq$ Aut $X^{*}$ be a level-transitive, self-similar, regular branch group over $K$. Then any non-trivial normal subgroup of $G$ contains $X^{n} * K^{\prime}$ for all $n$ big enough.

Since the above lemma applies for $G$, the next two prove Proposition D.5.3.

Lemma D.5.6. The group $K^{\prime}$ contains $X^{n} *[K, G]$ for all $n$ big enough.

Proof. We shall prove the statement for $n=5$. Since $K^{\prime}$ contains $K^{\prime} \times K^{\prime}$, the claim follows.

$K^{\prime}$ is characteristic in $K$ which is normal in $G$, thus $K^{\prime}$ is normal in $G$. Hence it is sufficient to show that $1^{5} *[k, s]$ is in $K^{\prime}$ for all $k \in K$ and $s \in\{a, \beta, \gamma, \delta\}$. We define the following elements of $K$ :

$$
\begin{aligned}
g_{a} & =[a, \beta]^{4 \gamma \beta^{a}}, & g_{\beta} & =[\gamma, \delta]^{4}, \\
g_{\gamma} & =[\beta, \delta]^{4 \beta^{a}}, & g_{\delta} & =[a, \beta]^{4 \gamma} .
\end{aligned}
$$

Then $g_{s} \in \operatorname{Stab}_{K}\left(1^{5}\right)$ and $\left(g_{s}\right) @ 1^{5}=s$ for all $s \in\{a, \beta, \gamma, \delta\}$. Therefore $\left[1^{5} *\right.$ $\left.k, g_{s}\right]=1^{5} *[k, s]$ is in $K^{\prime}$ for all $k \in K$ and $s \in\{a, \beta, \gamma, \delta\}$.

Lemma D.5.7. The group $[K, G]$ contains $X * C$.

Proof. Since $C$ is a subgroup of $K$, and $C$ is normal in $G$ and generated by $[K, G]$ and $x=[a, \beta][\beta, \gamma]$, it is enough to show that $1 * x=\varphi(x)$ is contained in $[K, G]$. One can check that $1 * x$ is trivial in $G / \gamma_{3}$.

\section{D.5.2 The Kernel}

The congruence kernel of $G$ is non-trivial, and therefore $G$ does not have the congruence subgroup property:

Theorem D.5.8. The congruence kernel $\operatorname{ker}(\widehat{G} \rightarrow \bar{G})$ is isomorphic to $(\mathbb{Z} / 4 \mathbb{Z})\left[\left[X^{\omega}\right]\right]$ as a profinite $\bar{G}$-module.

Proof. We use the method presented in [BSZ09] to compute the kernel. It is proved that one can compute the congruence kernel as $A\left[\left[\bar{G} / \overline{G_{w}}\right]\right]$, where $A$ is the finite group $\bigcap_{n \geq 0} \varphi^{n}(K / C)$ and $\overline{G_{w}}$ is the stabilizer of a point of the boundary of the tree in $\bar{G}$. 
$K / C$ is a cyclic group of order 4 , and $\varphi$ induces the non-trivial automorphism of this group. Therefore $A \simeq \mathbb{Z} / 4 \mathbb{Z}$. Next, the action of $G$ by conjugation on $K /[K, G]$ is clearly trivial. Since $K / C$ is a quotient of that group, $G$ acts trivially on it. Therefore the action of $\bar{G}$ on the congruence kernel is given by the canonical action on the boundary $X^{\omega}$ of $X^{*}$, and the kernel is $(\mathbb{Z} / 4 \mathbb{Z})\left[\left[X^{\omega}\right]\right]$.

\section{D.6 Germs}

We construct a homomorphism from $G$ to a finite group, which does not factor through Aut $X^{n}$ for any $n$. More precisely, we give explicit functions $G \rightarrow \mathbb{F}_{2}$, where $\mathbb{F}_{2}$ is the finite field with two elements (endowed with the discrete topology), which are continuous for the profinite topology on $G$, but not for the congruence topology. This yields some insights about the germs of the action on the boundary of the tree.

Consider the group $C$ of Proposition D.4.11 and write $\Gamma=G / C$, a group of order $2^{6}$. Define, for all $n \in \mathbb{N}$, the homomorphism $\pi_{n}: G \rightarrow \Gamma$ ? Aut $X^{n}$ as $\psi: G \rightarrow G$ 々 Aut $X^{n}$ followed by the natural quotient map.

Theorem D.6.1. Every epimorphism from $G$ onto a finite group factors through $\pi_{n}$ for all $n$ big enough.

The explicit computation of the maps $\pi_{n}$ is a bit technical, and shall be given after the proof of Theorem D.6.11. Recall that $N=\{1, a, \beta, \gamma, \delta\}$ is the nucleus of $G$; see Definition D.4.3.

Lemma D.6.2. For all $g, h \in G$, there is an integer $n$ such that $g @ w, h @ w$ and $(g h) @ w$ all belong to $N$ for all words $w$ of length at least $n$.

Proof. By definition there is $n_{g}$ such that $g @ w$ is in $N$ for all $w$ of length at least $n_{g}$. Similarly there is $n_{h}$ for $h$ and $n_{g h}$ for $g h$. The claim obviously holds with $n=\max \left\{n_{g}, n_{h}, n_{g h}\right\}$.

Define the permutation $\tau$ of the set $\{\beta, \gamma, \delta\}$ as

$$
\beta \mapsto \gamma, \quad \gamma \mapsto \delta, \quad \delta \mapsto \beta,
$$

so that $\tau^{3}$ is the identity. To each $\xi \in\{\beta, \gamma, \delta\}$ we associate a function $G \rightarrow \mathbb{F}_{2}$ defined by

$$
[\xi](g)= \begin{cases}1 & \text { if } g=\xi, \\ 0 & \text { if } g \neq \xi \text { but } g \in N \\ \sum_{x \in X}\left[\xi^{\tau}\right](g @ x) & \text { otherwise. }\end{cases}
$$

It is clear that the relation

$$
[\xi](g)=\sum_{w \in X^{n}}\left[\xi^{\tau^{n}}\right](g @ w)
$$

holds for all $\xi \in\{\beta, \gamma, \delta\}, g \in G$, and all $n \geq 0$. 
The restriction of the function $[\xi]$ to $N$ is the characteristic function of $\xi$. We now prove that $[\xi](g)$ is the number (modulo 2) of occurrences of $\xi$ in a decomposition of $g$ as a product of elements of $N$. This statement is made precise in the following lemma.

Lemma D.6.3. Let $\xi$ be one of $\beta, \gamma, \delta$. Then $\xi: G \rightarrow \mathbb{F}_{2}$ is a well-defined group homomorphism.

Proof. The group $G$ is contracting, therefore the computation of $[\xi](g)$ only involves a finite number of steps; hence $[\xi]$ is well-defined.

Note that another way to compute $[\xi](g)$ is as follows. Choose a multiple $n$ of 3 such that $g @ w$ is in $N$ for all $w \in X^{n}$. Then $[\xi](g)$ is the number (modulo 2 ) of words $w$ of length $n$ such that $g @ w=\xi$. This is equivalent to the original definition of $[\xi]$ by Equation (D.6.1).

The fact that $[\xi]$ is a group homomorphism follows from the above observation. Because $N$ generates $G$ it is sufficient to show that for all $s \in N$ and $g \in G$ the relation $[\xi](s g)=[\xi](s)+[\xi](g)$ holds. In other words we must show that the following holds for all $s \in N$ and $g \in G$ :

$$
[\xi](s g)-[\xi](g)= \begin{cases}1 & \text { if } s=\xi \\ 0 & \text { otherwise }\end{cases}
$$

Consider $s \in N$ and $g \in G$ and let $n$ be a multiple of 3 big enough so that $s @ w, g @ w$ and $(s g) @ w$ are all in $N$ for all $w \in X^{n}$ (cf. Lemma D.6.2). Note that we have the relation

$$
(s g) @ v=(s @ v)\left(g @ v^{s}\right) .
$$

If $s \neq \xi$ it is clear that $s @ w$ is different from $\xi$ for all $w$ of length $n$, and therefore $[\xi](g)=[\xi](s g)$. If $s=\xi$ then there is exactly one word $v$ of length $n$ such that $s @ v=\xi$. Then $[\xi]((s g) @ w)=[\xi]\left(g @ w^{s}\right)$ for all $w \neq v$ of length $n$, but we have $[\xi]((s g) @ v)=[\xi]\left(g @ v^{s}\right)+1(\bmod 2)$. Therefore $[\xi](s g)=\sum_{w \in X^{n}}[\xi]((s g) @ w)=$ $[\xi](g)+1(\bmod 2)$ in this case.

We define the notation $[x \xi](g)=[\xi](g @ x)$ for $x \in X, \xi \in\{\beta, \gamma, \delta\}$ and $g \in G$. Also, we let $[\varnothing]: G \rightarrow \mathbb{F}_{2}$ be the canonical epimorphism $G \rightarrow G / \operatorname{Stab}_{G}(1)$, after identifying $G / \operatorname{Stab}_{G}(1)$ with the additive group of $\mathbb{F}_{2}$.

Lemma D.6.4. The following relations hold

\begin{tabular}{|c|cccc|}
\hline$f$ & $f(a g)-f(g)$ & $f(\beta g)-f(g)$ & $f(\gamma g)-f(g)$ & $f(\delta g)-f(g)$ \\
\hline$[\varnothing] \cdot[\beta]$ & {$[\beta](g)$} & {$[\varnothing](g)$} & 0 & 0 \\
{$[\varnothing] \cdot[\gamma]$} & {$[\gamma](g)$} & 0 & {$[\varnothing](g)$} & 0 \\
{$[\varnothing] \cdot[\delta]$} & {$[\delta](g)$} & 0 & 0 & {$[\varnothing](g)$} \\
\hline
\end{tabular}

for all $g \in G$.

Proof. These are straightforward consequences of the definitions and of LemmaD.6.3.

For example, we have $([\varnothing] \cdot[\beta])(a g)=([\varnothing](a)+[\varnothing](g)) \cdot([\beta](a)+[\beta](g))=$ $([\varnothing] \cdot[\beta])(g)+[\beta](g)$. 
D.6. Germs

Lemma D.6.5. The relation

$$
[x \xi](s g)=[x \xi](s)+[x \xi](g)+[\varnothing](s) \cdot\left[\xi^{\tau^{-1}}\right](g)
$$

holds for all $x \in X, \xi \in\{\beta, \gamma, \delta\}$ and $s, g \in G$.

Proof. We compute $[x \xi](s g)=[\xi]((s g) @ x)=[\xi]\left((s @ x)\left(g @ x^{s}\right)\right)=[\xi](s @ x)+$ $[\xi]\left(g @ x^{s}\right)=[x \xi](s)+\left[x^{s} \xi\right](g)$. The claim follows from the relation $[\xi](g)=$ $\sum_{x \in X}\left[x \xi^{\tau}\right](g)$.

In the following we write $\bar{x}=1-x$.

Corollary D.6.6. The relations $[x \xi](a g)=[\bar{x} \xi](g)$ and $[x \xi](a g)-[x \xi](g)=$ $\left[\xi^{\tau^{-1}}\right](g)$ hold for all $g \in G$.

Corollary D.6.7. The relation $[x \xi](s g)=[x \xi](g)+[x \xi](s)$ holds for all $s \in$ $\{\beta, \gamma, \delta\}$ and all $g \in G$.

Corollary D.6.8. The following relations hold

\begin{tabular}{|c|cccc|}
\hline$f$ & $f(a g)-f(g)$ & $f(\beta g)-f(g)$ & $f(\gamma g)-f(g)$ & $f(\delta g)-f(g)$ \\
\hline$[1 \gamma]$ & {$[\beta](g)$} & 0 & 0 & 0 \\
{$[0 \delta]$} & {$[\gamma](g)$} & 0 & 0 & 0 \\
{$[0 \beta]$} & {$[\delta](g)$} & 0 & 0 & 0 \\
\hline
\end{tabular}

for all $g \in G$.

Define recursively the following functions

$$
\begin{aligned}
f_{1}(g)= & {[0 \delta](g)+[1 \gamma](g)+[0 \delta](g) \cdot[1 \delta](g)+\sum_{x \in X}\left([x \beta](g) \cdot[\bar{x} \delta](g)+f_{2}(g @ x)\right), } \\
f_{2}(g)= & {[0 \beta](g)+[1 \gamma](g)+[0 \gamma](g) \cdot[1 \gamma](g)+\sum_{x \in X} f_{3}(g @ x), } \\
f_{3}(g)= & {[0 \delta](g)+[1 \gamma](g)+[0 \gamma](g) \cdot[1 \gamma](g) } \\
& +\sum_{x \in X}\left([x \beta](g) \cdot[\bar{x} \gamma](g)+[x \beta](g) \cdot[\bar{x} \delta](g)+f_{1}(g @ x)\right) .
\end{aligned}
$$

Lemma D.6.9. The functions $f_{i}: G \rightarrow \mathbb{F}_{2}$ are well-defined for all $i \in\{1,2,3\}$.

Proof. This follows again form the fact that $G$ is contracting. Indeed, each of the functions $f_{i}$ consists of infinitely many terms. On the other hand it is easy to see that $f_{i}(g)=0$ for all $g \in N$ because all the terms of these infinite sums vanish. Therefore the evaluation of $f_{i}(g)$ only involves finitely many non-zero terms.

Proposition D.6.10. The following relations hold

\begin{tabular}{|c|cccc|}
\hline$f$ & $f(a g)-f(g)$ & $f(\beta g)-f(g)$ & $f(\gamma g)-f(g)$ & $f(\delta g)-f(g)$ \\
\hline$f_{1}$ & {$[\beta](g)+[\gamma](g)$} & {$[\gamma](g)+[\delta](g)$} & 0 & {$[\gamma](g)$} \\
$f_{2}$ & {$[\beta](g)+[\delta](g)$} & {$[\delta](g)$} & {$[\beta](g)+[\delta](g)$} & 0 \\
$f_{3}$ & {$[\beta](g)+[\gamma](g)$} & 0 & {$[\beta](g)$} & {$[\beta](g)+[\gamma](g)$} \\
\hline
\end{tabular}

for all $g \in G$. 
Proof. We begin with the first column, using Corollary D.6.6. The quadratic terms of each $f_{i}$ contribute to nothing in $f_{i}(a g)-f_{i}(g)$. Moreover, $\sum_{x \in X} f_{i}((a g) @$ $x)=\sum_{x \in X} f_{i}\left(@ x^{a}\right)=\sum_{x \in X} f_{i}(@ x)$, and therefore this term also gives no contribution. The linear terms clearly yield the result.

We use Corollary D.6.7 to prove the other nine relations. The proof goes by induction, using the fact that $G$ is contracting. We compute $f_{1}(\beta g)-f_{1}(g)$ as follows. We note that $[x \xi](\beta)=0$ unless $x=0$ and $\xi=\gamma$. Therefore we have

$$
f_{1}(\beta g)-f_{1}(g)=f_{2}(\gamma \cdot g @ 0)-f_{2}(g @ 0)+f_{2}(a \cdot g @ 1)-f_{2}(g @ 1) .
$$

Now we already computed $f_{2}(a \cdot g @ 1)-f_{2}(g @ 1)=([\beta]+[\delta])(g @ 1)=([1 \beta]+$ $[1 \delta])(g)$. Thus we get

$$
\begin{aligned}
f_{1}(\beta g)-f_{1}(g) & =[1 \beta](g)+[1 \delta](g)+f_{2}(\gamma \cdot g @ 0)-f_{2}(g @ 0) \\
& =[\delta](g)+[\gamma](g)+f_{2}(\gamma \cdot g @ 0)-f_{2}(g @ 0)-[\beta](g @ 0)-[\delta](g @ 0) .
\end{aligned}
$$

This is exactly what we wanted, if the relation $f_{2}(\gamma g)-f_{2}(g)=[\beta](g)+[\delta](g)$ holds. In the same way, one shows that the latter holds provided $f_{3}(\delta g)-f_{3}(g)=$ $[\beta](g)+[\gamma](g)$ holds. This, in turn, holds if $f_{1}(\beta g)-f_{1}(g)=[\gamma](g)+[\delta](g)$ holds, and we are back where we started. For the other relations, the induction step follows in a similar same way.

Thus, we are proving all the relations in a cyclic manner. For the basis of the induction it is enough to consider the case $g \in N$. Better, we only need to compute $f_{i}(s g)-f_{i}(g)$ for all $s, g \in N$ so that $s g$ is in $N$ (cf. Lemma D.6.2). Now $f_{i}(s g)-f_{i}(g)$ is obviously 0 if $g$ is trivial, because $f_{i}(s)=0$ for all $s \in N$. The only case left is $s=g$, and it is clear that $f_{i}(s g)-f_{i}(g)$ also vanishes in this situation.

Theorem D.6.11. Let $\Gamma$ be the nilpotent group of order $2^{6}$ given by the following presentation:

$\Gamma=\left\langle a, b, c, d \mid a^{2}, b^{2}, c^{2}, d^{2},[a, b]^{2},[a, c]^{2},[a, b]=[b, c]=[c, d]=[d, b],[a, d]=[a, b][a, c]\right\rangle$,

and consider the map $\pi: G \rightarrow \Gamma$ defined by $\pi(g)=a^{[\varnothing](g)} b^{[\beta](g)} c^{[\gamma](g)} d^{[\delta](g)}[a, b]^{e(g)}[a, c]^{f(g)}$ with $e(g)=\left(f_{3}+[\varnothing] \cdot([\beta]+[\delta])+[0 \beta]+[0 \delta]\right)(g)$ and $f(g)=([\varnothing] \cdot([\gamma]+[\delta])+[0 \beta]+$ $[0 \delta])(g)$. Then $\pi$ is a surjective group homomorphism. Moreover, the kernel of $\pi$ is $C$.

Proof. The identity is mapped to the identity under $\pi$. Thus it is enough to show that $\pi$ is multiplicative. Using Lemma D.6.4, Corollary D.6.8 and Proposition D.6.10, it is straightforward to check that we have the relations

\begin{tabular}{|c|cccc|}
\hline$\phi$ & $\phi(a g)-\phi(g)$ & $\phi(\beta g)-\phi(g)$ & $\phi(\gamma g)-\phi(g)$ & $\phi(\delta g)-\phi(g)$ \\
\hline$e$ & 0 & {$[\varnothing](g)$} & {$[\beta](g)$} & $([\beta]+[\gamma]+[\varnothing])(g)$ \\
$f$ & 0 & 0 & {$[\varnothing](g)$} & {$[\varnothing](g)$} \\
\hline
\end{tabular}

for all $g \in G$. Therefore $\pi$ is a group homomorphism, since $a, \beta, \gamma, \delta$ generate $G$ as a semigroup.

One can check that $\pi$ is surjective, and that $C$ is mapped to the trivial group. Since $C$ has index $2^{6}$ in $G$, the theorem is proved. 
Proof of Theorem D.6.1. By the previous theorem, the kernel of $\pi_{n}$ is $X^{n} * C$. Any finite-index subgroup contains $X^{n} * C$ for all $n$ big enough, by Proposition D.5.3.

\section{D.7 The Lower Central Series}

Let $\gamma_{1}=G$ and $\gamma_{n+1}=\left[G, \gamma_{n}\right]$ be the terms of the lower central series of $G$.

Proposition D.7.1. We have, for all $n \geq 3$ :

$$
\gamma_{2 n-1}=\gamma_{n} \times \gamma_{n}, \quad \gamma_{2 n}=\left\{\left(g, g^{-1}\right): g \in \gamma_{n}\right\} \gamma_{2 n+1} .
$$

We first prove two lemmas and then the proposition. Define $\bar{\gamma}_{2 n-1}=\gamma_{n} \times \gamma_{n}$ and $\bar{\gamma}_{2 n}=\left\{\left(g, g^{-1}\right): g \in \gamma_{n}\right\} \bar{\gamma}_{2 n+1}$ for $n \geq 3$. The groups $\bar{\gamma}_{k}$ are subgroups of $G$, because $K$ contains $\gamma_{3}$ (see Proposition D.4.8). Notice that the groups $\bar{\gamma}_{k}$ form a descending filtration in $G$.

Lemma D.7.2. We have the inclusion $\left[\bar{\gamma}_{k}, G\right] \subseteq \bar{\gamma}_{k+1}$ for $k \geq 5$. More precisely, for all $h \in \operatorname{Stab}_{G}(1)$ we have $\left[\bar{\gamma}_{k}, h\right] \subseteq \bar{\gamma}_{k+2}$ and $\left[\bar{\gamma}_{k}, a\right] \subseteq \bar{\gamma}_{k+1}$ if $k$ is odd, and $\left[\bar{\gamma}_{k}, h\right] \subseteq \bar{\gamma}_{k+1}$ and $\left[\bar{\gamma}_{k}, a\right] \subseteq \bar{\gamma}_{k+2}$ if $k$ is even.

Proof. Suppose $k$ is odd and write $k=2 n-1$. Consider $g=\left(g_{0}, g_{1}\right) \in \gamma_{n} \times \gamma_{n}=\bar{\gamma}_{k}$ and $h=\left(h_{0}, h_{1}\right) \in \operatorname{Stab}_{G}(1)$. Then obviously $[g, h]=\left(\left[g_{0}, h_{0}\right],\left[g_{1}, h_{1}\right]\right) \in \bar{\gamma}_{2 n+1}$ and $[g, a]=\left(g_{0}^{-1} g_{1},\left(g_{0}^{-1} g_{1}\right)^{-1}\right) \in \bar{\gamma}_{2 n}$.

Suppose $k$ is even and write $k=2 n$. Consider $g \in \gamma_{n}$ and $h=\left(h_{0}, h_{1}\right) \in$ $\operatorname{Stab}_{G}(1)$. Then obviously $\left[\left(g, g^{-1}\right), h\right]=\left(\left[g, h_{0}\right],\left[g^{-1}, h_{1}\right]\right)$ is in $\bar{\gamma}_{2 n+1}$. Next, since $G$ is generated by involutions, the quotients in the lower central series are elementary Abelian 2-groups. This implies that $g^{2} \in \gamma_{n+1}$ and thus $\left[\left(g, g^{-1}\right), a\right]=$ $\left(g^{-2}, g^{2}\right) \in \bar{\gamma}_{2 n+2}$. Notice that we already proved the inclusions $\left[\bar{\gamma}_{2 n+1}, h\right] \subseteq \bar{\gamma}_{2 n+1}$ and $\left[\bar{\gamma}_{2 n+1}, a\right] \subseteq \bar{\gamma}_{2 n+2}$, so we are done.

Lemma D.7.3. For all $n \geq 3$, we have the inclusion $\left[\bar{\gamma}_{2 n-1}, G\right] \supseteq \bar{\gamma}_{2 n}$; for all $n \geq 3$, if the equality $\bar{\gamma}_{k}=\gamma_{k}$ holds for all $5 \leq k \leq n+2$, then we have the inclusion $\left[\bar{\gamma}_{2 n}, G\right] \supseteq \bar{\gamma}_{2 n+1}$.

Proof. It is clear that $\left[\bar{\gamma}_{2 n-1}, G\right]$ contains $[(1, g), a]=\left(g, g^{-1}\right)$ for all $g \in \gamma_{n}$. Since $G$ is recurrent, we have $\left[\bar{\gamma}_{2 n-1}, G\right] \supseteq \gamma_{n+1} \times \gamma_{n+1}=\bar{\gamma}_{2 n+1}$. Therefore $\left[\bar{\gamma}_{2 n-1}, G\right]$ contains $\bar{\gamma}_{2 n}$ and we are done for the first statement.

Suppose now $\bar{\gamma}_{k}=\gamma_{k}$ holds for all $5 \leq k \leq n+2$. By definition, $\bar{\gamma}_{2 n}$ contains $\bar{\gamma}_{2 n+1}$. Therefore $\left[\bar{\gamma}_{2 n}, G\right]$ contains $\left[\bar{\gamma}_{2 n+1}, G\right] \supseteq \bar{\gamma}_{2 n+2} \supseteq \gamma_{n+2} \times \gamma_{n+2}$. We now prove that, modulo $\gamma_{n+2} \times \gamma_{n+2}$, the group $\left[\bar{\gamma}_{2 n}, G\right]$ contains $([g, s], 1)$ for all $g \in \gamma_{n}$ and all $s \in\{a, \beta, \gamma, \delta\}$, and this will conclude the proof. A direct computation shows that $\left[\bar{\gamma}_{2 n}, G\right]$ contains the elements

$$
\begin{aligned}
& {\left[\left(g, g^{-1}\right), \beta\right]=\left([g, \gamma],\left[g^{-1}, a\right]\right), \quad\left[\left(g, g^{-1}\right), \gamma\right]=\left([g, a],\left[g^{-1}, \delta\right]\right),} \\
& {\left[\left(g, g^{-1}\right), \delta\right]=\left(1,\left[g^{-1}, \beta\right]\right)}
\end{aligned}
$$

for all $g \in \gamma_{n}$. Using Lemmas D.7.2 and D.9.2, we see that either $[g, a]$ or $\left[g^{-1}, \beta\right],[g, \gamma],\left[g^{-1}, \delta\right]$ are in $\gamma_{n+2}$, so we are done. 
Proof of Proposition D.7.1. We need to prove the equality $\bar{\gamma}_{k}=\gamma_{k}$ for all $k \geq 5$. Note that if we prove this for all $5 \leq k \leq n$, then Lemmata D.7.2 and D.7.3 show that $\left[\bar{\gamma}_{k}, G\right]=\bar{\gamma}_{k+1}$ for all $5 \leq k \leq 2 n-3$. In turn, this implies $\bar{\gamma}_{k}=\gamma_{k}$ for all $5 \leq k \leq 2 n-2$. Therefore, since $2 n-2>n$ for $n \geq 5$, we only need to prove the case $k=5$. This is done using GAP in Lemma D.9.3.

Remark D.7.4. The same proposition, with the same proof, holds for the Grigorchuk group. The only change is in Lemma D.9.3, where one needs to replace the presentation and the endomorphism by those from Equation (D.4.2).

It is convenient to introduce some notation. For a set $S \subseteq$ Aut $X^{*}$, we define $\Delta * S=\left\{\left(g, g^{-1}\right) \mid g \in S\right\}$ and $0 * S=\{(g, 1) \mid g \in S\}$. We then write $\Delta \Delta * S$ instead of $\Delta *(\Delta * S)$ and so on.

Corollary D.7.5. The group $G$ has finite width. More precisely, $\gamma_{n} / \gamma_{n+1}$ is a $(\mathbb{Z} / 2 \mathbb{Z})$-vector space, and

$$
\operatorname{rank}\left(\gamma_{n} / \gamma_{n+1}\right)= \begin{cases}2 & \text { if } \frac{3}{4} 2^{i}+1 \leq n \leq 2^{i} \text { for some } i \in \mathbb{N} \\ 4 & \text { otherwise. }\end{cases}
$$

Proof. Proposition D.7.1 can be rewritten as

$$
\gamma_{2 n-1}=X * \gamma_{n}, \quad \gamma_{2 n}=\left(\Delta * \gamma_{n}\right)\left(X * \gamma_{n+1}\right),
$$

for all $n \geq 3$. From this it is immediate that

$$
\gamma_{2 n} / \gamma_{2 n+1} \simeq \gamma_{2 n-1} / \gamma_{2 n} \simeq \gamma_{n} / \gamma_{n+1}
$$

holds for all $n \geq 3$. Therefore $G$ has finite width. Even more is true: if $S_{n}$ is a minimal generating set for $\gamma_{n}$ modulo $\gamma_{n+1}$, then $0 * S_{n}$ is a minimal generating set for $\gamma_{2 n-1}$ modulo $\gamma_{2 n}$, and $\Delta * S_{n}$ is a minimal generating set for $\gamma_{2 n}$ modulo $\gamma_{2 n+1}$. Therefore we obtain the following sequence

$$
\begin{aligned}
S_{1}, S_{2}, S_{3}, S_{4}, \\
\quad 0 * S_{3}, \Delta * S_{3}, 0 * S_{4}, \Delta * S_{4}, \\
\quad 00 * S_{3}, \Delta 0 * S_{3}, 0 \Delta * S_{3}, \Delta \Delta * S_{3}, 00 * S_{4}, \Delta 0 * S_{4}, 0 \Delta * S_{4}, \Delta \Delta * S_{4}, \ldots
\end{aligned}
$$

of minimal generating sets for $\gamma_{n}$ (modulo $\gamma_{n+1}$ ). Explicitly, we compute the rank and a minimal generating set for $\gamma_{n} / \gamma_{n+1}$ in Lemma D.9.4, for $1 \leq n \leq 4$. It follows that the rank of $\gamma_{n} / \gamma_{n+1}$ is given by

$$
4,4,4,2,4,4,2,2,4,4,4,4,2,2,2,2, \ldots
$$




\section{D.8. The Schur Multiplier}

\section{D.8 The Schur Multiplier}

In this section we compute the second homology group of $G$, also known as the Schur multiplier $M(G)=H_{2}(G, \mathbb{Z})$. Since it has infinite rank, the group $G$ cannot be finitely presented.

An important novelty of our computation - in addition to making it as algorithmic as possible - is the description of $M(G)$ as a finitely presented module over $\mathbb{Z}[\varphi]$, where $\varphi$ is the group endomorphism defined in Theorem D.4.1.

Let $\Gamma$ be the group given by the presentation $\left\langle a, \beta, \gamma, \delta \mid a^{2}, \beta^{2}, \gamma^{2}, \delta^{2}\right\rangle$ and let $\Omega$ be the kernel of the map $\Gamma \rightarrow G$ sending generators to generators. Let $\varphi$ be the endomorphism of $\Gamma$ induced by the one in Theorem D.4.1. Write $r_{1}^{(0)}=\left[\delta^{a}, \delta\right]$, $r_{2}^{(0)}=\left[\delta, \gamma^{a} \beta\right], r_{3}^{(0)}=\left[\delta,\left(\gamma^{a} \beta\right)^{\gamma}\right]$ and $r_{4}^{(0)}=\left[\gamma^{a} \beta, \gamma \beta^{a}\right]$, and $r_{i}^{(n)}=\varphi^{n}\left(r_{i}^{(0)}\right)$ for $n \geq 0$. Then the elements $r_{i}^{(n)}$ form a set of normal generators of $\Omega$ in $\Gamma$.

Using the "five-term homology sequence" [Rob96] applied on the extension $1 \rightarrow \Omega \rightarrow \Gamma \rightarrow G \rightarrow 1$, we get the following exact sequence:

$$
M(\Gamma) \rightarrow M(G) \rightarrow \Omega /[\Gamma, \Omega] \rightarrow \Gamma / \Gamma^{\prime} \rightarrow G / G^{\prime} \rightarrow 1
$$

Now $\Gamma / \Gamma^{\prime} \rightarrow G / G^{\prime}$ is an isomorphism, and the group $\Gamma$ is isomorphic to a free product of four copies of $\mathbb{Z} / 2 \mathbb{Z}$, whence $M(\Gamma)=0$. Thus we have an isomorphism $M(G) \simeq \Omega /[\Gamma, \Omega]$.

We shall write $M=M(G)=\Omega /[\Gamma, \Omega]$ and denote by $\bar{r}$ the image in $M$ of an element $r \in \Omega$.

Lemma D.8.1. The group $M$ is an elementary Abelian 2-group.

Proof. Since $\Omega \leq \Gamma$, the quotient $\Omega /[\Gamma, \Omega]$ is clearly Abelian. Moreover, the images of the elements $r_{i}^{(0)}$ and of their iterates under $\varphi$ generate $M$. Therefore it is enough to show that $r_{i}$ is conjugate to its inverse for $i=1,2,3,4$. A direct computation yields

$$
\begin{aligned}
{\left[\delta, \delta^{a}\right] } & =\left[\delta^{a}, \delta\right]^{a}, & {\left[\gamma^{a} \beta, \delta\right] } & =\left[\delta, \gamma^{a} \beta\right]^{\delta}, \\
{\left[\left(\gamma^{a} \beta\right)^{\gamma}, \delta\right] } & =\left[\delta,\left(\gamma^{a} \beta\right)^{\gamma}\right]^{\delta}, & {\left[\gamma \beta^{a}, \gamma^{a} \beta\right] } & =\left[\gamma^{a} \beta, \gamma \beta^{a}\right]^{a},
\end{aligned}
$$

thus $M$ is an elementary Abelian 2-group.

Let $\psi: \Gamma \rightarrow \Gamma$ 乙 $C_{2}$ be the lift of the wreath decomposition of $G$ defined by

$$
\psi(a)=\sigma, \quad \psi(\beta)=(\gamma, a), \quad \psi(\gamma)=(a, \delta), \quad \psi(\delta)=(1, \beta) .
$$

Lemma D.8.2. We have the inclusion $\psi([\Gamma, \Omega]) \leq([\Gamma, \Omega] \times[\Gamma, \Omega])\left\{\left(r, r^{-1}\right) \mid r \in\right.$ $\Omega\}$.

Proof. We know that the map $\psi$ descends to $G$. Therefore $\psi(\Omega) \leq \Omega \times \Omega$. Let $\Sigma \leq \Gamma$ be the subgroup of index 2 generated by $\left\{\beta, \gamma, \delta, \beta^{a}, \gamma^{a}, \delta^{a}\right\}$. Then clearly $\psi([\Sigma, \Omega]) \leq[\Gamma, \Omega] \times[\Gamma, \Omega]$. Finally, write $\psi(r)=\left(r_{1}, r_{2}\right)$ for $r \in \Omega$. Then $\psi([a, r])=\left(r_{2}^{-1} r_{1}, r_{1}^{-1} r_{2}\right)$, so we proved the claim. 
Let $\Delta$ be the subgroup $\left\{\left(\bar{r}, \overline{r^{-1}}\right) \mid r \in \Omega\right\}$ of $M \times M$. This is a subgroup since $M$ is Abelian. Then the group $(M \times M) / \Delta$ is naturally isomorphic to $M$. Thus by the above lemma, the map $\psi: \Omega \rightarrow \Omega \times \Omega$ induces a map $\Psi: M \rightarrow$ $(M \times M) / \Delta \simeq M$. This endomorphism can be computed as follows. Let $\bar{r}$ be an element of $M$ and choose a lift $r \in \Omega$. Then $\Psi(\bar{r})$ is the image of $\psi(r) \in \Omega \times \Omega$ modulo $([\Gamma, \Omega] \times[\Gamma, \Omega])\left\{\left(r, r^{-1}\right) \mid r \in \Omega\right\}$.

Lemma D.8.3. We have the isomorphism $M=\operatorname{ker} \Psi \times \Psi(M)$.

Proof. We clearly have the exact sequence $1 \rightarrow \operatorname{ker} \Psi \rightarrow M \rightarrow \Psi(M) \rightarrow 1$. Since $M$ is elementary Abelian, the extension is a direct sum.

Let $\bar{R}$ be the subgroup of $M$ generated by $\overline{r_{1}^{(0)}}$. The following lemma explains the behaviour of $\Psi$.

Lemma D.8.4. For all $i=1,2,3,4$ and $n>0$, the map $\Psi$ sends $\overline{r_{i}^{(n)}}$ to $\overline{r_{i}^{(n-1)}}$, modulo $\bar{R}$.

Proof. Consider $r_{i}^{(n)}=\varphi\left(r_{i}^{(n-1)}\right)$ with $n>0$. Let $D \leq \Gamma$ be the infinite dihedral group generated by $a$ and $\delta$. Then $\psi\left(r_{i}^{(n)}\right)$ equals $\left(1, r_{i}^{(n-1)}\right)$ modulo $D \times 1$. Moreover, since $\psi\left(r_{i}^{(n)}\right)$ is in $\Omega \times \Omega$, we must have $\psi\left(r_{i}^{(n)}\right)=\left(1, r_{i}^{(n-1)}\right)$ modulo $R \times 1$ with $R=D \cap \Omega=\left\langle r_{1}^{(0)}\right\rangle$. This last equality holds because $a$ and $\delta$ generate a dihedral group of order 8 in $G$. Thus we proved the equality $\Psi\left(\overline{r_{i}^{(n)}}\right)=\overline{r_{i}^{(n-1)}}$ modulo $\bar{R}$.

Lemma D.8.5. The group $N_{0} \leq M$ generated by $\left\{\overline{r_{1}^{(0)}}, \overline{r_{2}^{(0)}}, \overline{r_{3}^{(0)}}, \overline{r_{4}^{(0)}}, \overline{r_{1}^{(1)}}\right\}$ is in the kernel of $\Psi$.

Proof. For $i=1,2,3,4$, the element $\psi\left(r_{i}^{(0)}\right)$ is trivial in $\Gamma \times \Gamma$ as a direct computation shows. Another straightforward computation yields $\psi\left(r_{1}^{(1)}\right) \equiv\left(r_{1}^{(0)}, r_{1}^{(0)}\right)$ modulo $[\Gamma, \Omega] \times[\Gamma, \Omega]$, and thus $r_{1}^{(1)}$ is also in the kernel of $\Psi$.

Proposition D.8.6. The elements $\overline{r_{i}^{(n)}}$ are all independent in $M$, and $\Psi\left(r_{i}^{(n)}\right)$ is non-trivial unless $r_{i}^{(n)}$ is in $N_{0}$.

Proof. For $n>0$, let $N_{n} \leq M$ be the subgroup generated by $\left\{\overline{r_{2}^{(n)}}, \overline{r_{3}^{(n)}}, \overline{r_{4}^{(n)}}, \overline{r_{1}^{(n+1)}}\right\}$. Note that by Lemma D.9.5, the group $N_{0}$ is isomorphic to $(\mathbb{Z} / 2 \mathbb{Z})^{5}$.

Lemma D.8.4 shows that for all $n>0$, we have $\Psi\left(\overline{r_{i}^{(n)}}\right)=\overline{r_{i}^{(n-1)}}$ modulo $\bar{R}$ for $i \in\{2,3,4\}$ and similarly $\Psi\left(\overline{r_{1}^{(n+1)}}\right)=\overline{r_{1}^{(n)}}$ modulo $\bar{R}$. Thus $\Psi\left(N_{n}\right)=N_{n-1}$, modulo $\bar{R}$.

We now prove by induction that for all $n>0$, the group $N_{n}$ has rank 4 and trivial intersection with $N_{0} \cdots N_{n-1}$. For the case $n=1$, we note the isomorphism $N_{0} / \bar{R} \simeq(\mathbb{Z} / 2 \mathbb{Z})^{4}$. Since $\Psi\left(N_{1}\right)=N_{0}$ modulo $\bar{R}$, it follows that $N_{1}$ has rank 4. And $N_{1}$ has trivial intersection with $N_{0}$ because $\Psi\left(N_{0}\right)=0$ and $\Psi\left(N_{1}\right) \simeq$ $(\mathbb{Z} / 2 \mathbb{Z})^{4}$. 


\section{D.9. GAP Computations}

Now suppose the induction hypothesis holds for some $n>0$. Then $N_{n} \bar{R} / \bar{R}$ has rank 4 . We have $\Psi\left(N_{n+1}\right)=N_{n}$ modulo $\bar{R}$, and therefore $N_{n+1}$ has rank 4 . Next $\Psi^{n+1}\left(N_{0} \cdots N_{n}\right)=0$, whereas $\Psi^{n+1}\left(N_{n+1}\right)=N_{0}$ modulo $\bar{R}$. This proves that $\Psi^{n+1}\left(N_{n+1}\right) \simeq(\mathbb{Z} / 2 \mathbb{Z})^{4}$ and so $N_{n+1}$ has trivial intersection with $N_{0} \cdots N_{n}$. Therefore the $\overline{r_{i}^{(n)}}$ are all independent.

Corollary D.8.7. The kernel of $\Psi$ is $N_{0} \simeq(\mathbb{Z} / 2 \mathbb{Z})^{5}$.

The endomorphism $\varphi$ of $\Gamma$ induces an endomorphism on $G$. Therefore $\varphi(\Omega) \leq$ $\Omega$ and $\varphi$ induces an endomorphism (which we still write $\varphi$ ) on $M$ defined by $\bar{r} \mapsto \overline{\varphi(r)}$. Thus one can consider $M$ as a $(\mathbb{Z} / 2 \mathbb{Z})[\varphi]$-module.

Lemma D.8.8. The endomorphism $\varphi$ is a right inverse of $\Psi$ modulo $\bar{R}$.

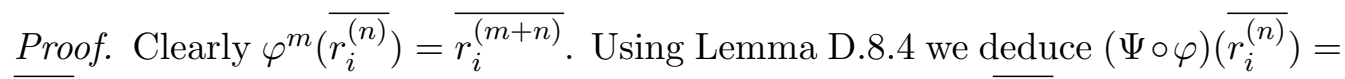
$\overline{r_{i}^{(n)}}$ modulo $\bar{R}$, for all $i=1,2,3,4$ and $n \geq 0$. Since the $\overline{r_{i}^{(n)}}$ generate $M$, the claim is proved.

We summarize our results in the following

Theorem D.8.9. The Schur multiplier $M(G)$ is isomorphic to a direct sum of four copies of $(\mathbb{Z} / 2 \mathbb{Z})[\varphi]$. More precisely, a set of $(\mathbb{Z} / 2 \mathbb{Z})[\varphi]$-independent generators of $M(G)$ is $\left\{\overline{r_{1}^{(0)}}, \overline{r_{2}^{(0)}}, \overline{r_{3}^{(0)}}, \overline{r_{4}^{(0)}}\right\}$.

Remark D.8.10. For the Grigorchuk group, the situation is slightly more subtle. First, one proves that the Schur multiplier is elementary Abelian "by hand", as in Lemma D.8.1.

Then, as auxiliary group, one uses $\Gamma=\left\langle a, b, c, d \mid a^{2}, b^{2}, c^{2}, d^{2}, b c d\right\rangle \simeq(\mathbb{Z} / 2 \mathbb{Z}) *$ $(\mathbb{Z} / 2 \mathbb{Z})^{2}$. The reason for this is that otherwise $b c d$ would be fixed by $\Psi$. The Schur multiplier of $\Gamma$ is $\mathbb{Z} / 2 \mathbb{Z}$, and using the five-term homology sequence we get the extension $1 \rightarrow \mathbb{Z} / 2 \mathbb{Z} \rightarrow M(G) \rightarrow \Omega /[\Gamma, \Omega] \rightarrow 1$. The rest of the proof is absolutely similar to the above, and one finally gets $M(G) \simeq \mathbb{Z} / 2 \mathbb{Z} \times((\mathbb{Z} / 2 \mathbb{Z})[\varphi])^{2}$, which one could pedantically write as $M(G) \simeq((\mathbb{Z} / 2 \mathbb{Z})[\varphi] /(\varphi-1)) \times((\mathbb{Z} / 2 \mathbb{Z})[\varphi])^{2}$, see [Gri99].

\section{D.9 GAP Computations}

Lemma D.9.1. The L-presentations

(i) $\left\langle a, \beta, \gamma, \delta|\tilde{\varphi}| a^{2}, \beta^{2}, \gamma^{2}, \delta^{2},\left[\delta^{a}, \delta\right],\left[\delta, \gamma^{a} \beta\right],\left[\delta,\left(\gamma^{a} \beta\right)^{\gamma}\right],\left[\gamma^{a} \beta, \gamma \beta^{a}\right]\right\rangle$ and

(ii) $\left\langle a, \beta, \gamma, \delta|\tilde{\varphi}| a^{2}, \beta^{2}, \gamma^{2}, \delta^{2},\left[\delta^{a}, \delta^{w}\right],\left[\delta^{a},\left(\gamma \beta^{a}\right)^{w}\right],\left[\gamma \beta^{a},\left(\gamma \beta^{a}\right)^{w}\right]\right\rangle$, with $w \in\left\{1, \gamma, \gamma^{a}, \gamma \gamma^{a}, \gamma^{a} \gamma, \gamma \gamma^{a} \gamma, \gamma^{a} \gamma \gamma^{a}, \gamma \gamma^{a} \gamma \gamma^{a}\right\}$

define the same group.

Proof. Clearly, all the relations in (i) are relations in (ii). Therefore it is enough to check that the normal closure of the relations in (i) together with some of their iterates under $\tilde{\varphi}$ contains all the relations of (ii). This is done via the following commands using GAP, together with the package KBMAG [KBMAG09]: 


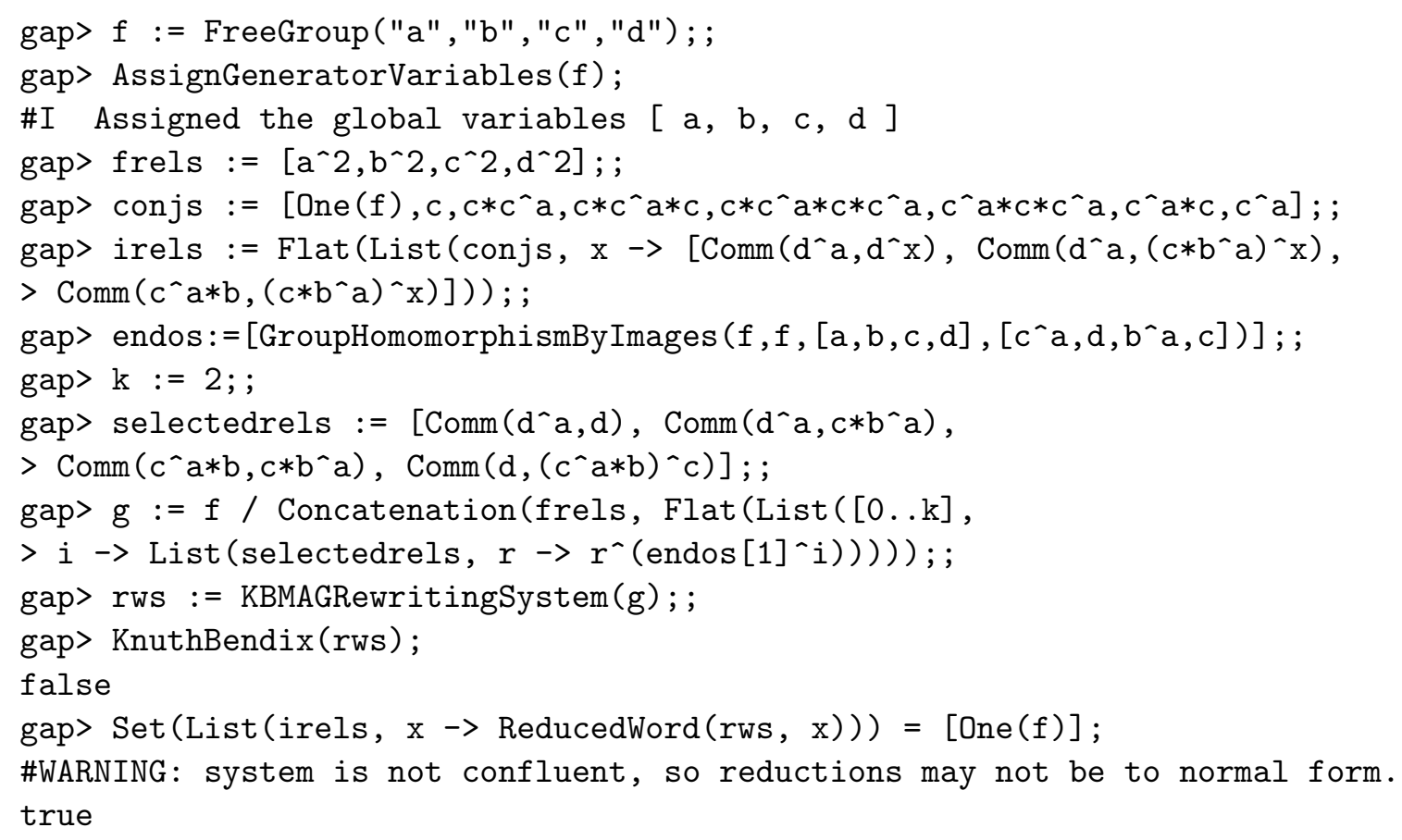

We do not require the Knuth-Bendix procedure to terminate. Indeed, the command KnuthBendix stops automatically after a few seconds, once it has computed a few thousand new rules. At this point, the rewriting system already contains enough rules to prove that all the words of irels are congruent to the identity.

Let $\gamma_{n}$ be the $n$-th term of the lower central series of $G$.

Lemma D.9.2. The inclusion $\left[\gamma_{3},\{\beta, \gamma, \delta\}\right] \subseteq \gamma_{5}$ and $\left[\gamma_{4}, a\right] \subseteq \gamma_{6}$ hold.

Proof. It is enough to work in $G / \gamma_{6}$, and then to check:

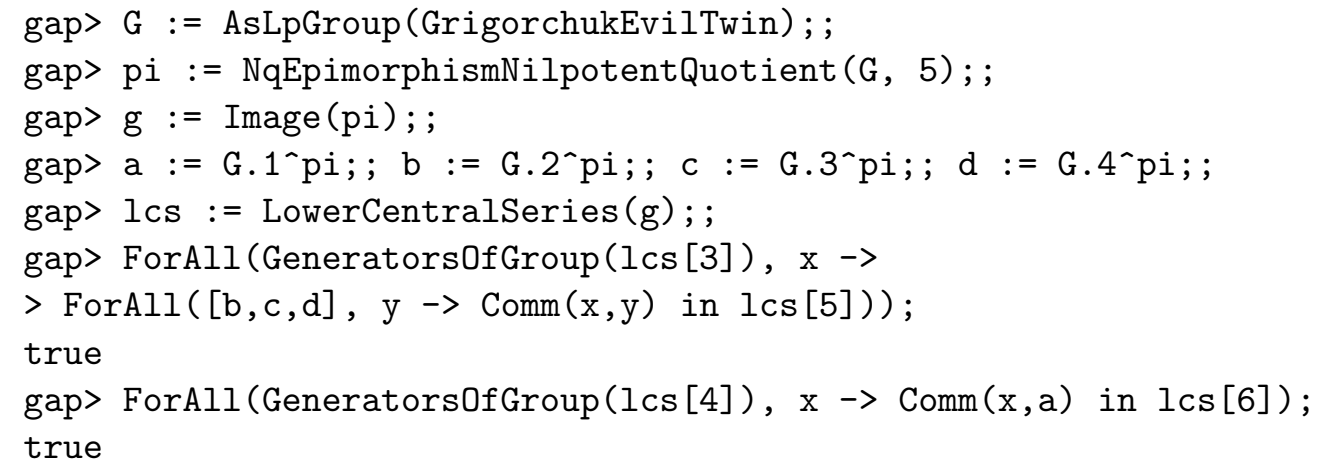

Lemma D.9.3. We have the equality $\gamma_{5}=\gamma_{3} \times \gamma_{3}$.

Proof. Let $G=F /\left\langle R_{\infty}\right\rangle^{F}$ be a presentation of $G$, where $R$ is a finite set, and $R_{\infty}=\bigcup_{n \geq 0} \tilde{\varphi}^{n}(R)$ (see Theorem D.4.1). We compute a lift $\tilde{S}$ in $F$ of a set $S$ 


\section{D.9. GAP Computations}

of normal generators of $\gamma_{3}$. By Proposition D.4.8, $\gamma_{3}$ is a subgroup of $K$, and therefore $\varphi\left(\gamma_{3}\right)=1 \times \gamma_{3}$. Thus $\gamma_{3} \times \gamma_{3}$ is the normal closure of $\varphi\left(\gamma_{3}\right)$, and $\varphi(S)$ is a set of normal generators of $\gamma_{3} \times \gamma_{3}$. Therefore $F /\left\langle R_{\infty} \cup \tilde{\varphi}(\tilde{S})\right\rangle^{F}$ is a presentation of $G /\left(\gamma_{3} \times \gamma_{3}\right)$. This observation allows us to verify that the group $G /\left(\gamma_{3} \times \gamma_{3}\right)$ has nilpotency class (at most) 4:

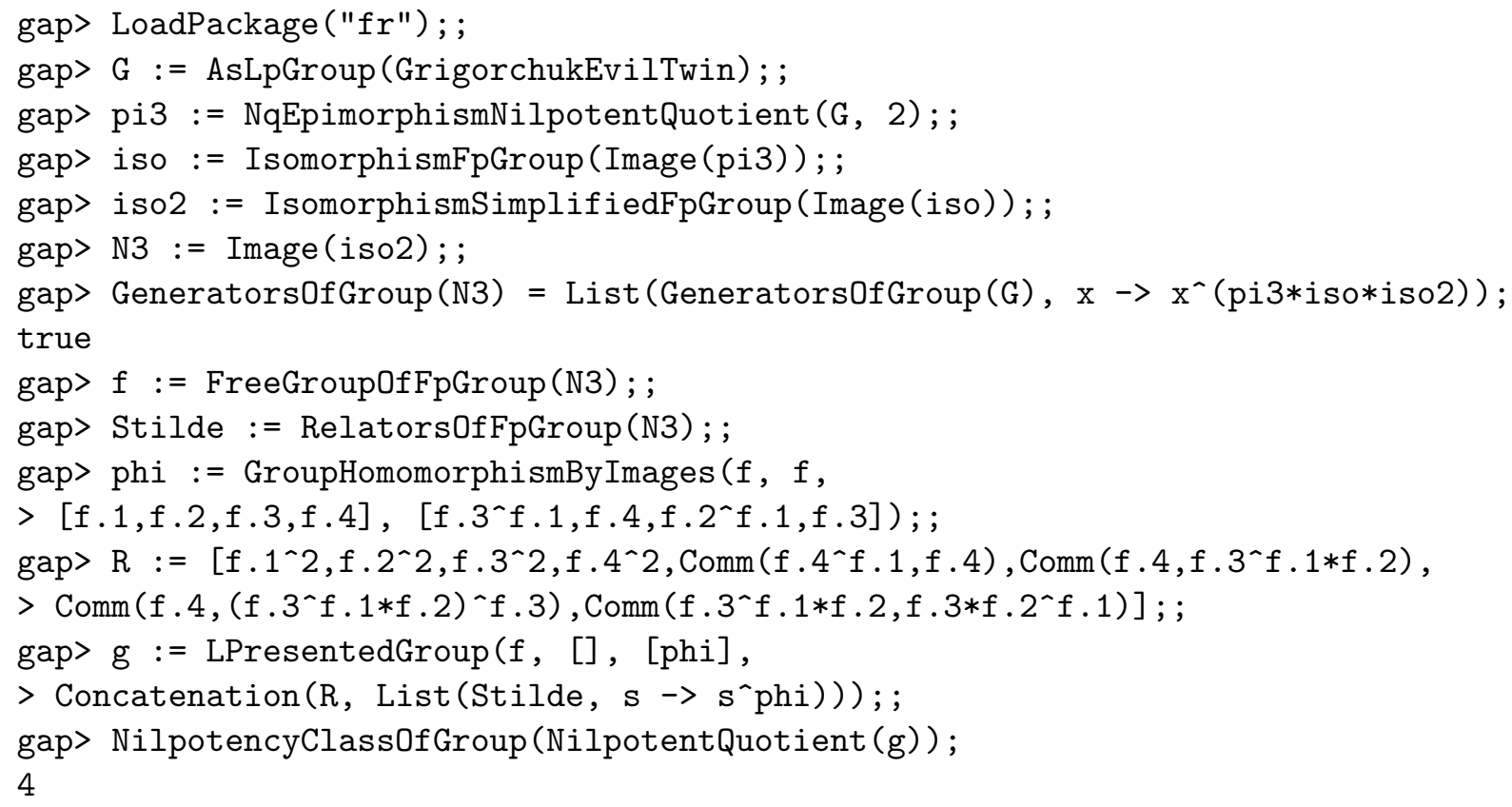

The attentive reader will notice that we compute $G /\left(\gamma_{3} \times \gamma_{3}\right) \simeq F /\left\langle(R \cup \tilde{\varphi}(\tilde{S}))_{\infty}\right\rangle^{F}$, but the equality $\left\langle(R \cup \tilde{\varphi}(\tilde{S}))_{\infty}\right\rangle^{F}=\left\langle R_{\infty} \cup \tilde{\varphi}(\tilde{S})\right\rangle^{F}$ holds because $\varphi\left(\gamma_{3}\right)$ is a subgroup of $\gamma_{3}$, and thus $\tilde{\varphi}(\tilde{\varphi}(\tilde{S}))$ is contained in $\left\langle R_{\infty} \cup \tilde{\varphi}(\tilde{S})\right\rangle^{F}$.

Further, we can now easily prove the inclusion $\gamma_{5} \geq \gamma_{3} \times \gamma_{3}$. We simply need to check

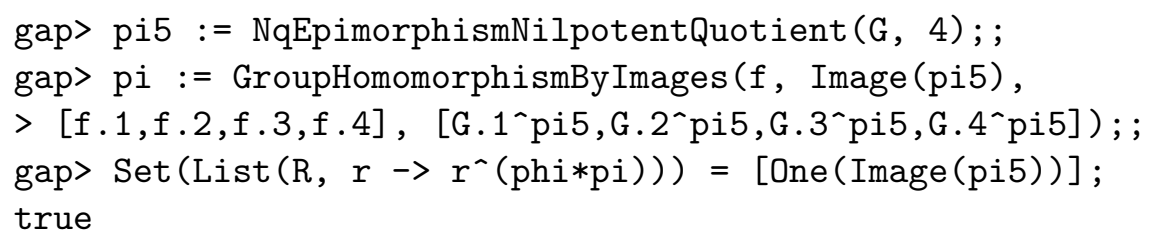

Lemma D.9.4. The rank of $\gamma_{n} / \gamma_{n+1}$ is $4,4,4,2$ for $n=1,2,3,4$ respectively. Moreover, minimal generating sets are given in the following table:

\begin{tabular}{|c|l|}
\hline$n$ & generating set for $\gamma_{n} / \gamma_{n+1}$ \\
\hline 1 & $a, \beta, \gamma, \delta$ \\
2 & {$[a, \beta],[a, \gamma],[a, \delta],[\beta, \gamma]$} \\
3 & {$[[a, \beta], a],[[a, \beta], \gamma],[[a, \beta], \delta],[[a, \gamma], a]$} \\
4 & {$[[[a, \beta], \gamma], a],[[[a, \beta], \delta], a]$} \\
\hline
\end{tabular}

Proof. We compute in $G / \gamma_{5}$ : 


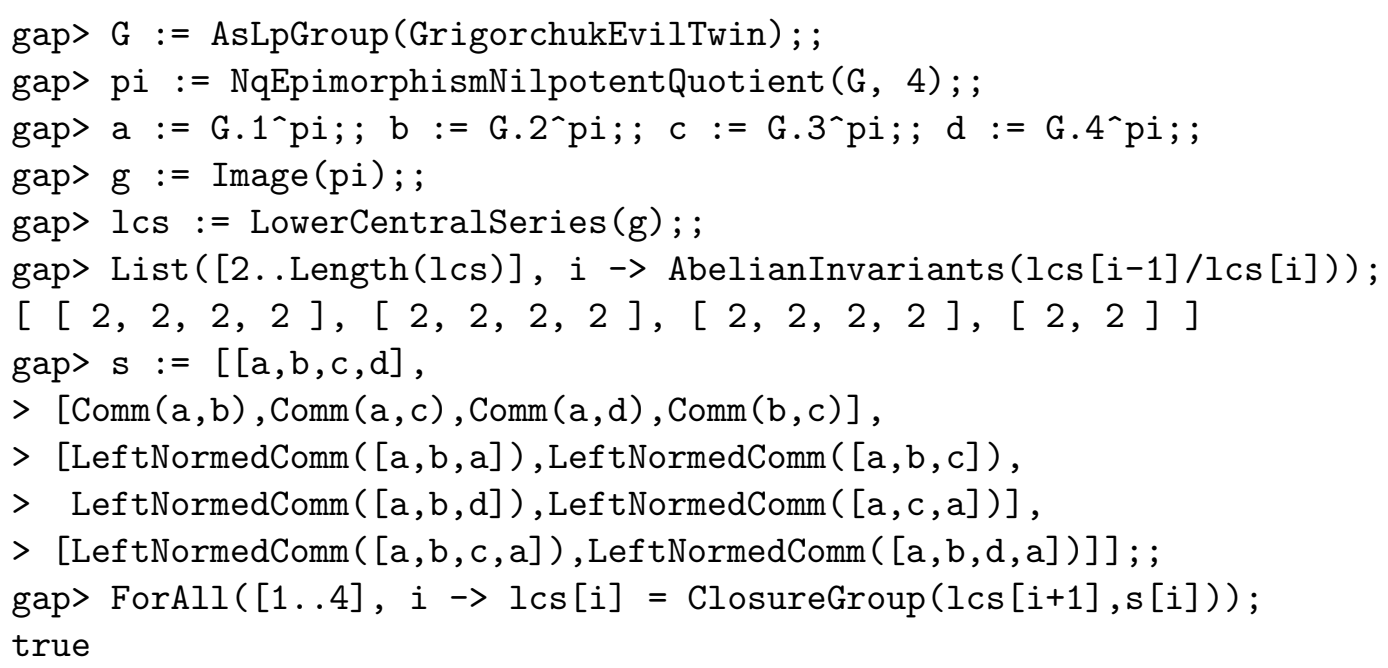

For the following lemma the notations are as in Section D.8.

Lemma D.9.5. The elements $\left\{\overline{r_{1}^{(0)}}, \overline{r_{2}^{(0)}}, \overline{r_{3}^{(0)}}, \overline{r_{4}^{(0)}}, \overline{r_{1}^{(1)}}\right\}$ generate a group isomorphic to $(\mathbb{Z} / 2 \mathbb{Z})^{5}$ in the group $\Omega /\left([\Gamma, \Omega] \varphi^{2}(\Omega)\left(\gamma_{8}(\Gamma) \cap \Omega\right)\right)$.

Proof. We first define the group gam $=\Gamma /\left([\Gamma, \Omega] \varphi^{2}(\Omega)\right)$ as an $L$-presented group using the package NQL [NQL09].

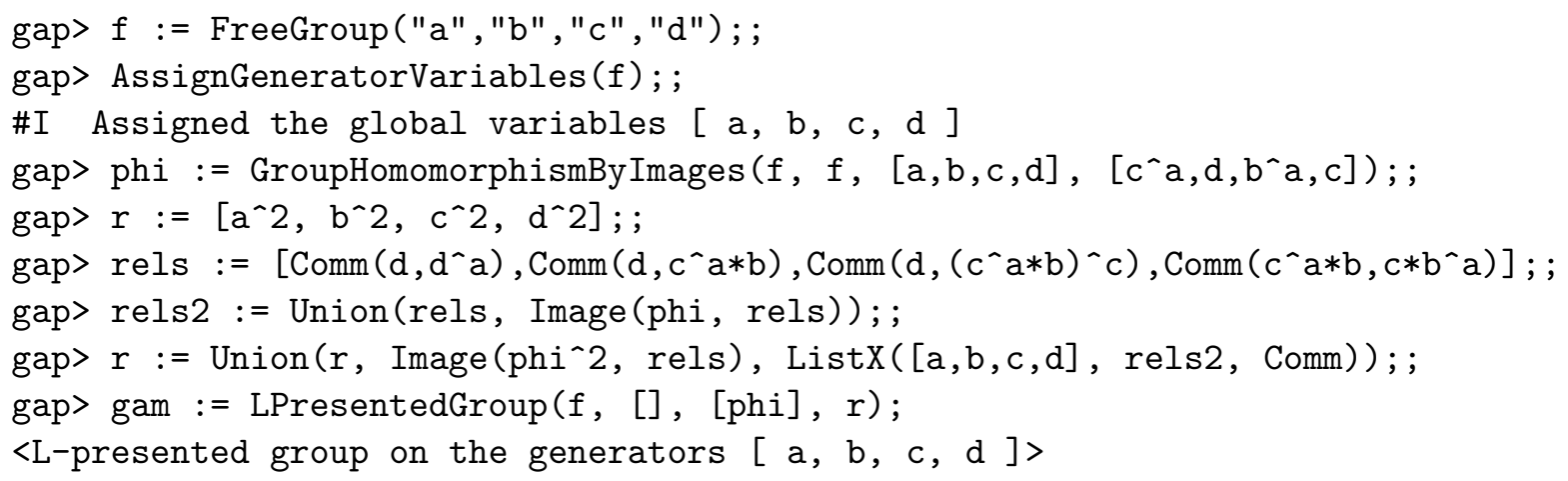

Then we kill the eighth term of the lower central series of gam, and look at the group generated by the images of $\left\{\overline{r_{1}^{(0)}}, \overline{r_{2}^{(0)}}, \overline{r_{3}^{(0)}}, \overline{r_{4}^{(0)}}, \overline{r_{1}^{(1)}}\right\}$.

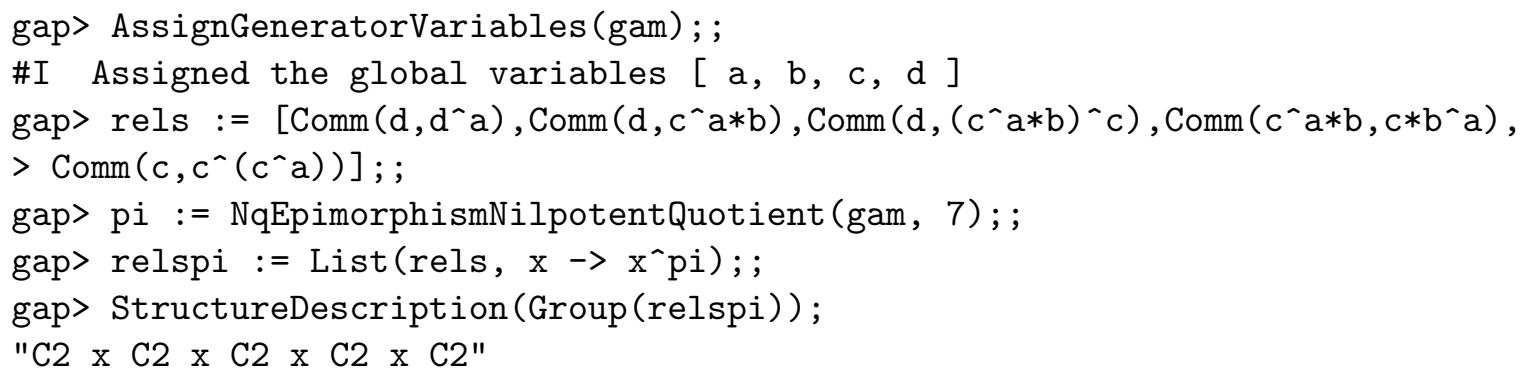





\section{Bibliography}

[Abe94] A. G. Abercrombie, Subgroups and subrings of profinite rings, Math. Proc. Cambridge Philos. Soc. 116 (1994), no. 2, 209-222. MR 1281541 (95h:11078)

[Abé05] Miklós Abért, Group laws and free subgroups in topological groups, Bull. London Math. Soc. 37 (2005), no. 4, 525-534. MR 2143732 (2006d:20005)

[AV05] Miklós Abért and Bálint Virág, Dimension and randomness in groups acting on rooted trees, J. Amer. Math. Soc. 18 (2005), no. 1, 157-192 (electronic). MR 2114819 (2005m:20058)

[Ale72] S. V. Alešin, Finite automata and the Burnside problem for periodic groups, Mat. Zametki 11 (1972), 319-328 (Russian); English transl., Math. Notes 11 (1972), 199-203. MR 0301107 (46 \#265)

[AAV09] Gideon Amir, Omer Angel, and Bálint Virág, Amenability of linear-activity automaton groups, 2009, preprint.

[AHKŠ07] Goulnara Arzhantseva, Pierre de la Harpe, Delaram Kahrobaei, and Zoran Šunić, The true prosoluble completion of a group: examples and open problems, Geom. Dedicata 124 (2007), 5-26. With an appendix by Arzhantseva and Šunić. MR 2318535 (2008e:20038)

[BS97] Yiftach Barnea and Aner Shalev, Hausdorff dimension, pro-p groups, and KacMoody algebras, Trans. Amer. Math. Soc. 349 (1997), no. 12, 5073-5091. MR 1422889 (98b:20041)

[Bar03] Laurent Bartholdi, Endomorphic presentations of branch groups, J. Algebra 268 (2003), no. 2, 419-443. MR 2009317 (2004h:20044)

[Bar05] _ Lie algebras and growth in branch groups, Pacific J. Math. 218 (2005), no. 2, 241-282. MR 2218347 (2007k:20069)

[Bar03] _ A Wilson group of non-uniformly exponential growth, C. R. Math. Acad. Sci. Paris 336 (2003), no. 7, 549-554 (English, with English and French summaries). MR 1981466 (2004c:20051)

[BEH08] Laurent Bartholdi, Bettina Eick, and René Hartung, A nilpotent quotient algorithm for certain infinitely presented groups and its applications, Internat. J. Algebra Comput. 18 (2008), no. 8, 1321-1344. MR 2483125

[BG00] Laurent Bartholdi and Rostislav I. Grigorchuk, Lie methods in growth of groups and groups of finite width, Computational and geometric aspects of modern algebra (Edinburgh, 1998), London Math. Soc. Lecture Note Ser., vol. 275, Cambridge Univ. Press, Cambridge, 2000, pp. 1-27. MR 1776763 (2001h:20046)

[BG02] On parabolic subgroups and Hecke algebras of some fractal groups, Serdica Math. J. 28 (2002), no. 1, 47-90. MR 1899368 (2003c:20027)

[BGŠ03] Laurent Bartholdi, Rostislav I. Grigorchuk, and Zoran Šuniḱ, Branch groups, Handbook of algebra, Vol. 3, North-Holland, Amsterdam, 2003, pp. 989-1112. MR 2035113 (2005f:20046)

[BKN08] Laurent Bartholdi, Vadim A. Kaimanovich, and Volodymyr V. Nekrashevych, On amenability of automata groups (2008), submitted, available at arXiv.org/abs/0802.2837. 


\section{Bibliography}

[BS06] Laurent Bartholdi and Said N. Sidki, The automorphism tower of groups acting on rooted trees, Trans. Amer. Math. Soc. 358 (2006), no. 1, 329-358 (electronic). MR 2171236 (2006i:20036)

[BS09] Laurent Bartholdi and Olivier Siegenthaler, The Twisted Twin of the Grigorchuk Group, 2009, submitted.

[BSZ09] Laurent Bartholdi, Olivier Siegenthaler, and Pavel Zalesskii, The congruence subgroup problem for branch groups, 2009, submitted.

[BŠ01] Laurent Bartholdi and Zoran Šunik, On the word and period growth of some groups of tree automorphisms, Comm. Algebra 29 (2001), no. 11, 4923-4964. MR 1856923 (2002i:20040)

[BV05] Laurent Bartholdi and Bálint Virág, Amenability via random walks, Duke Math. J. 130 (2005), no. 1, 39-56. MR 2176547 (2006h:43001)

[BLS64] Hyman Bass, Michel Lazard, and Jean-Pierre Serre, Sous-groupes d'indice fini dans SL $(n, \mathbf{Z})$, Bull. Amer. Math. Soc. 70 (1964), 385-392 (French). MR 0161913 (28 \#5117)

[BORT96] Hyman Bass, Maria Victoria Otero-Espinar, Daniel Rockmore, and Charles Tresser, Cyclic renormalization and automorphism groups of rooted trees, Lecture Notes in Mathematics, vol. 1621, Springer-Verlag, Berlin, 1996. MR 1392694 (97k:58058)

[Bha95] Meenaxi Bhattacharjee, The ubiquity of free subgroups in certain inverse limits of groups, J. Algebra 172 (1995), no. 1, 134-146. MR 1320624 (96c:20044)

[Bor69] Armand Borel, Linear algebraic groups, Notes taken by Hyman Bass, W. A. Benjamin, Inc., New York-Amsterdam, 1969. MR 0251042 (40 \#4273)

[OWR06] Pro-p extensions of global fields and pro-p groups, Oberwolfach Rep. 3 (2006), no. 2, 1463-1535. Abstracts from the workshop held May 21-27, 2006; Organized by Nigel Boston, John Coates and Fritz Grunewald; Oberwolfach Reports, Vol. 3, no. 2. MR 2308272

[Bou61] N. Bourbaki, Algèbre commutative, Actualités Scientifiques et Industrielles, Hermann, Paris, 1961 (French). MR 0171800 (30 \#2027)

[BSV99] A. M. Brunner, Said Sidki, and Ana Cristina Vieira, A just nonsolvable torsionfree group defined on the binary tree, J. Algebra 211 (1999), no. 1, 99-114. MR 1656573 (99m:20054)

[H00] Pierre de la Harpe, Topics in geometric group theory, Chicago Lectures in Mathematics, University of Chicago Press, Chicago, IL, 2000. MR 1786869 (2001i:20081)

[EP84] M. Edjvet and Stephen J. Pride, The concept of "largeness" in group theory. II, Groups-Korea 1983 (Kyoungju, 1983), Lecture Notes in Math., vol. 1098, Springer, Berlin, 1984, pp. 29-54. MR 781355 (86g:20039)

[FG85] Jacek Fabrykowski and Narain Gupta, On groups with sub-exponential growth functions, J. Indian Math. Soc. (N.S.) 49 (1985), no. 3-4, 249-256 (1987). MR 942349 (90e:20029)

[FG91] _ On groups with sub-exponential growth functions. II, J. Indian Math. Soc. (N.S.) 56 (1991), no. 1-4, 217-228. MR 1153150 (93g:20053)

[GAP08] The GAP Group, GAP - Groups, Algorithms, and Programming, Version 4.4.10, 2008.

[Gri80] R. I. Grigorčuk, On Burnside's problem on periodic groups, Funktsional. Anal. i Prilozhen. 14 (1980), no. 1, 53-54 (Russian); English transl., Functional Anal. Appl. 14 (1980), no. 1, 41-43. MR 565099 (81m:20045)

[Gri83] R. I. Grigorchuk, On the Milnor problem of group growth, Dokl. Akad. Nauk SSSR 271 (1983), no. 1, 30-33 (Russian); English transl., Soviet Math. Dokl. 28 (1983), no. 1, 23-26. MR 712546 (85g:20042) 
[Gri00] _ Just infinite branch groups, New horizons in pro-p groups, Progr. Math., vol. 184, Birkhäuser Boston, Boston, MA, 2000, pp. 121-179. MR 1765119 (2002f:20044)

[Gri05] Rostislav Grigorchuk, Solved and unsolved problems around one group, Infinite groups: geometric, combinatorial and dynamical aspects, Progr. Math., vol. 248, Birkhäuser, Basel, 2005, pp. 117-218. MR 2195454 (2007d:20001)

[Gri99] Rostislav I. Grigorchuk, On the system of defining relations and the Schur multiplier of periodic groups generated by finite automata, Groups St. Andrews 1997 in Bath, I, London Math. Soc. Lecture Note Ser., vol. 260, Cambridge Univ. Press, Cambridge, 1999, pp. 290-317. MR 1676626 (2001g:20034)

[GŠ06] Rostislav Grigorchuk and Zoran Šunik, Asymptotic aspects of Schreier graphs and Hanoi Towers groups, C. R. Math. Acad. Sci. Paris 342 (2006), no. 8, 545-550 (English, with English and French summaries). MR 2217913 (2006k:20048)

[Gro60] A. Grothendieck, Éléments de géométrie algébrique. I. Le langage des schémas, Inst. Hautes Études Sci. Publ. Math. (1960), no. 4, 228 (French). MR 0163908 (29 \#1207)

[GS83a] Narain Gupta and Saïd Sidki, On the Burnside problem for periodic groups, Math. Z. 182 (1983), no. 3, 385-388. MR 696534 (84g:20075)

[GS83b] N. Gupta and Said Sidki, Some infinite p-groups, Algebra i Logika 22 (1983), no. 5, 584-589 (English, with Russian summary). MR 759409 (85k:20102)

[NQL09] René Hartung, NQL - Nilpotent Quotients of L-Presented Groups, Version 0.06, 2009 .

[Hoc81] Gerhard P. Hochschild, Basic theory of algebraic groups and Lie algebras, Graduate Texts in Mathematics, vol. 75, Springer-Verlag, New York, 1981. MR 620024 (82i:20002)

[Hoc71] G. Hochschild, Introduction to affine algebraic groups, Holden-Day Inc., San Francisco, Calif., 1971. MR 0277535 (43 \#3268)

[KBMAG09] Derek Holt, KBMAG - Knuth-Bendix on Monoids, and Automatic Groups, Version 1.5, 2009.

[HU79] John E. Hopcroft and Jeffrey D. Ullman, Introduction to automata theory, languages, and computation, Addison-Wesley Series in Computer Science, AddisonWesley Publishing Co., Reading, Mass., 1979.

[Jac79] Nathan Jacobson, Lie algebras, Dover Publications Inc., New York, 1979. Republication of the 1962 original. MR 559927 (80k:17001)

[Kal95] Léo Kaloujnine, Sur la structure des p-groupes de Sylow des groupes symétriques finis et de quelques généralisations infinies de ces groupes, Séminaire Bourbaki, Vol. 1, Soc. Math. France, Paris, 1995, pp. Exp. No. 5, 29-31 (French). MR 1605168

[LN02] Yaroslav Lavreniuk and Volodymyr Nekrashevych, Rigidity of branch groups acting on rooted trees, Geom. Dedicata 89 (2002), 159-179. MR 1890957 (2003i:20042)

[Lys85] I. G. Lysënok, A set of defining relations for the Grigorchuk group, Mat. Zametki 38 (1985), no. 4, 503-516, 634 (Russian); English transl., Math. Notes 38 (1985), no. 3-4, 784-792. MR 819415 (87g:20062)

[Mil68] John W. Milnor, Problem 5603, Amer. Math. Monthly 75 (1968), 685-686.

[MM65] John W. Milnor and John C. Moore, On the structure of Hopf algebras, Ann. of Math. (2) 81 (1965), 211-264. MR 0174052 (30 \#4259)

[Nek05] Volodymyr Nekrashevych, Self-similar groups, Mathematical Surveys and Monographs, vol. 117, American Mathematical Society, Providence, RI, 2005. MR 2162164 (2006e:20047)

[Nek07] _ A minimal Cantor set in the space of 3-generated groups, Geom. Dedicata 124 (2007), 153-190. MR 2318543 (2008d:20075) 


\section{Bibliography}

[Neu86] Peter M. Neumann, Some questions of Edjvet and Pride about infinite groups, Illinois J. Math. 30 (1986), no. 2, 301-316. MR 840129 (88a:20039)

[Per07] Ekaterina L. Pervova, Profinite completions of some groups acting on trees, J. of Algebra 310 (2007), no. 2, 858-879.

[Ple83] Wilhelm Plesken, Permutation groups with uniserial modules, Bull. London Math. Soc. 15 (1983), no. 1, 51-55. MR 686349 (84d:20011)

[RZ00] Luis Ribes and Pavel Zalesskii, Profinite groups, A Series of Modern Surveys in Mathematics, vol. 40, Springer-Verlag, Berlin, 2000. MR 1775104 (2001k:20060)

[Rob96] Derek J. S. Robinson, A course in the theory of groups, 2nd ed., Graduate Texts in Mathematics, vol. 80, Springer-Verlag, New York, 1996. MR 1357169 (96f:20001)

[Roz96] A. V. Rozhkov, Lower central series of a group of tree automorphisms, Mat. Zametki 60 (1996), no. 2, 225-237, 319 (Russian, with Russian summary); English transl., Math. Notes 60 (1996), no. 1-2, 165-174 (1997). MR 1429123 (98b:20048)

[Seg04] D. Segal, On the finite images of infinite groups, Groups: topological, combinatorial and arithmetic aspects, London Math. Soc. Lecture Note Ser., vol. 311, Cambridge Univ. Press, Cambridge, 2004, pp. 542-563. MR 2073358 (2005k:20062)

[Sel51] Ernst S. Selmer, The Diophantine equation $a x^{3}+b y^{3}+c z^{3}=0$, Acta Math. 85 (1951), 203-362 (1 plate). MR 0041871 (13,13i)

[Sid00] Said Sidki, Automorphisms of one-rooted trees: growth, circuit structure, and acyclicity, J. Math. Sci. (New York) 100 (2000), no. 1, 1925-1943. Algebra, 12. MR 1774362 (2002g:05100)

[Sid87] _ On a 2-generated infinite 3-group: the presentation problem, J. Algebra 110 (1987), no. 1, 13-23. MR 904179 (89b:20081a)

[Sie08] Olivier Siegenthaler, Hausdorff dimension of some groups acting on the binary tree, J. Group Theory 11 (2008), no. 4, 555-567. MR 2429355 (2009g:20046)

[Šun07] Zoran Šunić, Hausdorff dimension in a family of self-similar groups, Geom. Dedicata 124 (2007), 213-236. MR 2318546 (2008d:20046)

[Suš79] V. I. Suščans'kiı̌, Periodic p-groups of permutations and the unrestricted Burnside problem, Dokl. Akad. Nauk SSSR 247 (1979), no. 3, 557-561 (Russian). MR 545692 (81k:20009)

[SN05] V. I. Sushchanskiĭ and N. V. Netreba, Lie algebras associated with Sylow psubgroups of finite symmetric groups, Mat. Stud. 24 (2005), no. 2, 127-138 (Russian, with English and Russian summaries). MR 2223999 (2007c:17026)

[Swe69] Moss E. Sweedler, Hopf algebras, Mathematics Lecture Note Series, W. A. Benjamin, Inc., New York, 1969. MR 0252485 (40 \#5705)

[Wat79] William C. Waterhouse, Introduction to affine group schemes, Graduate Texts in Mathematics, vol. 66, Springer-Verlag, New York, 1979. MR 547117 (82e:14003)

[Wil04] John S. Wilson, On exponential growth and uniformly exponential growth for groups, Invent. Math. 155 (2004), no. 2, 287-303. MR 2031429 (2004k:20085)

[Wil98] _ Profinite groups, London Mathematical Society Monographs. New Series, vol. 19, The Clarendon Press Oxford University Press, New York, 1998. MR 1691054 (2000j:20048) 


\section{Curriculum Vitæ}

\section{Olivier Siegenthaler}

Title: $\quad$ Ingénieur Physicien (ing. phys. dipl. EPF)

Date of birth: 7th December 1983

Citizenship: Swiss

Address: $\quad$ Hofhölzliweg 4, CH-8046 Zürich

\section{Education}

2005 - 2009 Ph.D. Division of Mathematics and Natural Sciences Thesis Advisor: Laurent Bartholdi

Georg-August-Universität Göttingen

Eidgenössische Technische Hochschule Zürich (ETHZ)

Ecole Polytechnique Fédérale de Lausanne (EPFL)

2000 - $2005 \quad$ Master of Science en Physique

Thesis Advisor: Hervé Kunz

Ecole Polytechnique Fédérale de Lausanne (EPFL)

1999 - $2000 \quad$ Maturité Fédérale ès Sciences (type C)

Ecole Samos, Lausanne

\section{Awards}

$2005 \quad$ Youngest graduate of the year

Ecole Polytechnique Fédérale de Lausanne (EPFL)

\section{Publications}

2009 The Congruence Subgroup Problem for Branch Groups with Laurent Bartholdi and Pavel Zalesskii

submitted

2009 The Twisted Twin of the Grigorchuk Group with Laurent Bartholdi accepted by the International Journal of Algebra and Computation

2008 Hausdorff Dimension of Some Groups Acting on the Binary Tree Journal of Group Theory 11 (2008), no. 4, 555-567 